$$
\text { DOE/PC/90029-T T5 }
$$

APR \& 11992

\title{
Cooperative Research Program in Coal Liquefaction
}

DOE/PC/90029--T5

DE92 013722

\section{Quarterly Report on DOE Contract}

No. DE-FC22-90PC90029

\section{Period: August 1, 1991 - October 31, 1991}

Edited by G.P. Huffman

Director, Consortium for Fossil Fuel Liquefaction Science

University of Kentucky

233 Mining and Mineral Resources Bldg.

Lexington, Kentucky 40506-0107

(606) 257-4027

Submitted to the

U.S. Department of Energy

On behalf of the

Consortium for Fossil Fuel Liquefaction Science

University of Kentucky

University of Pittsburgh

University of Utah

West Virginia University

Auburn University 


\section{TABLE OF CONTENTS}

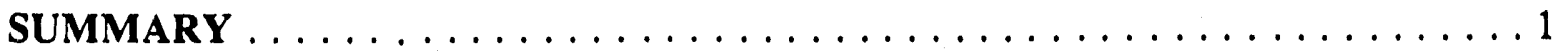

TASK I - Iron Based Catalysts for Coal Liquefaction $\ldots \ldots \ldots \ldots \ldots \ldots$

Project I.1 - Sulfated Iron Oxides as Coal Liquefaction Catalysts

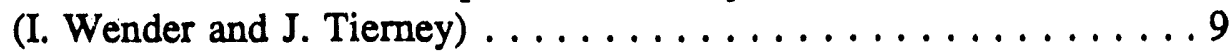

Project I.2 - Low Temperature Depolymerization and Liquefaction of

Premium U.S. Coal Samples

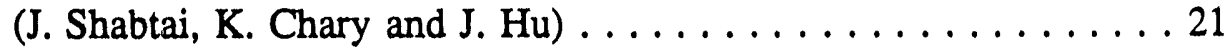

Project I.3 - Generic Structural Characterization and Liquefaction Research

(G. Huffman, N. Shah, J. Zhao, B. Ganguly, K. Rao,

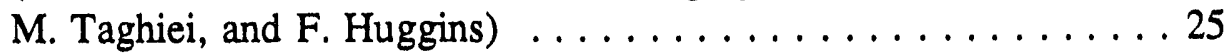

Project I.4 - Catalyst Dispersion and a Model Compound (E. Eyring, D. Sommerfeld, and Y.-H. Huang) $\ldots \ldots \ldots \ldots \ldots \ldots \ldots \ldots \ldots \ldots \ldots$

Project I.5 - Characterization of Catalysts Used in Coal Hydrogenation

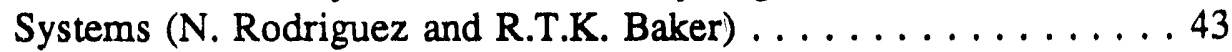

Project I.6 - Test Reactor for Catalytic Coal Liquefaction

(J. Zondlo, A. Stiller, P. Stansberry, D. Dadyburjor) . . . . . . . . 49

Project I.7 - Ferric Sulfide as a Precursor for Coal Liquefaction

and Surface Studies of Activity and Selectivity

(D. Dadyburjor, A. Stiller, J. Zondlo, P. Stansberry,

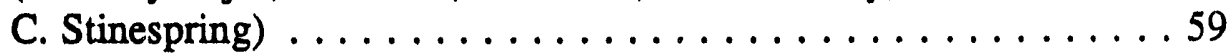

Project I.8 - Laser Pyrolysis Production of Ultrafine Particle Catalysts

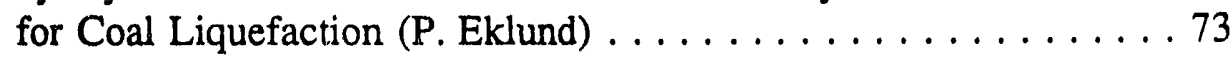

TASK II - Exploratory Research On Coal Conversion $\ldots \ldots \ldots \ldots \ldots \ldots 77$

Project II.1 - Coal Structure/Liquefaction Yield Correlation by

Means of Advanced NMR Techniques (R. Pugmire) . . . . . . . . 79

Project II.2 - Mass Spectrometric Analysis and Modeling of Coal

Liquefaction Processes (H. Meuzelaar and H. Huai) . . . . . 83 
Project II.3 - Catalytic Cracking/Hydrogenation and Catalysts Characterization using X-ray Diffraction and Squid Magnetometry (M. Seehra and M. Ibrahim) . . . . . . . . 89

Project II.4 - ESR and ENDOR Investigations for Coal Liquefaction Under Mild Conditions (N. Dalal and J. DeLooze) . . . . . . . . . . 97

Project II.5 - Depth Profile Studies on Catalyst and Catalyst Impregnated

Coal Samples (P. Reucroft and J. Kim) . . . . . . . . . . . 103

Project II.6 - Computational Chemistry of Model Compounds and Molecular Fragments of Relevance to Coal Liquefaction

(K. Subbaswarny) ....................... 113

Project II.7 - Chemical Characterization and Hydrogenation Reactions of Single Coal Particles (A. Ray) $\ldots \ldots \ldots \ldots \ldots \ldots \ldots 121$

Project II.8 - Liquefaction Pathways (B. Davis and R. Keogh) $\ldots \ldots \ldots \ldots \ldots 133$

Project II.9 - Development of Advanced Methods of Coal

Liquefaction Product Analysis (E. Kugler) . . . . . . . . . . . 139

TASK III - Novel Coal Liquefaction Concepts . . . . . . . . . . . . . 143

Project III.1 - Bioprocessing of Coal (D. Bhattacharyya, M. Murty, R. Kermode,

M. Venkatachalam, M. Aleem, K. Overstreet) . . . . . . . 145

Project III.2 - Distinguishing Chemical Reaction Mechanisms in Coal Liquefaction (J. Penn) $\ldots \ldots \ldots \ldots \ldots \ldots$

Project III.3 - Better Hydrogen Transfer in Coal Liquefaction

(A. Tarrer) . . . . . . . . . . . . . . . . . . 173

Project III.4 - (A) Catalytic Hydropyrolysis - Catalysts, Coals and

Liquid Product Quality; (B) Energized Coal

Liquefaction (L. Anderson, J. Jaturapitpornsakul, W.-H. Yuan) .

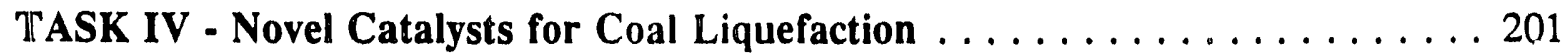

Project IV.1 - Catalytic Dehydrogenation of Model Compounds in Relation to Coal Liquefaction

(I. Wender, J. Tierney, G. Holder) . . . . . . . . . . . . . 203 
Project IV.2 - Evaluation of Dispersion Methods for Slurry Phase Catalysts

(Y. Tang and C. Curtis).

Project IV.3 - Improved Catalysts for Coal Liquefaction and Co-Processing:

New Initiative in Dispersed Slurry Phase Catalysts

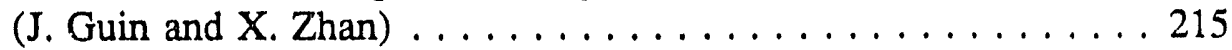




\section{SUMMARY}

\section{Iron-Based Catalysts for Coal Liquefaction}

A systematic study has been made of the kinetics of the direct liquefaction of Wyodak coal using three finely divided iron oxide-based catalysts: $\mathrm{Mo} / \mathrm{Fe}_{2} \mathrm{O}_{3} / \mathrm{SO}_{4}$, $\mathrm{Fe}_{2} \mathrm{O}_{3} / \mathrm{SO}_{4}$, and $\mathrm{Fe}_{2} \mathrm{O}_{3}$. The objective was to compare the activities of these finely dispersed iron-based catalysts on a quantitative basis. The kinetics of the direct liquefaction of Argonne Wyodak coal (\% C=75) using tetralin and 1000 psig (cold) H2 were experimentally measured and mathematically modeled. To develop the model, experiments were carried out in a $27 \mathrm{cc}$ horizontally shaken autoclave microreactor at $375-$ $425^{\circ} \mathrm{C}$ for reaction times of $0-120$ minutes. A simple lumped parameter model using oils, asphaltenes, coal, and gases as the product fractions was used to describe the observed kinetics. Model parameters include frequency factors and activation energies for four first order reactions, coal to gases, coal to oil, coal to asphaltenes, and asphaltenes to oils. This model successfully describes the effect of catalyst type and catalyst loading and indicates that the asphaltenes to oils reaction is the primary catalytic step. The model successfully predicts the effect of the non-isothermal heat-up period in a stirred autoclave and can be used to predict conversions at temperatures other than those used to determine the model parameters.

Systematic studies were continued on a premium Lewiston-Stockton (WV) coal with the purpose of determining suitable HT-BCD prucessing conditions for high-yield conversion of this feed into depolymerized products. For a selected set of conditions in the initial HT step (reaction temperature, $275^{\circ} \mathrm{C} ; \mathrm{H}_{2}$ pressure, $1500 \mathrm{psig}$; reaction time, 1 $\mathrm{h}$ ), the total yield of depolymerized (THF-soluble) products in the subsequent BCD step increases markedly with increase in $\mathrm{BCD}$ temperature (from $37.1 \mathrm{wt} \%$ at $250^{\circ} \mathrm{C}$ to 85.9 $w t \%$ at $305^{\circ} \mathrm{C}$ ). Furthermore, the proportion of the oil fraction in the solubilized product increases from $54.3 \mathrm{wt} \%$ at $250^{\circ} \mathrm{C}$ to $71.7 \mathrm{wt} \%$ at $305^{\circ} \mathrm{C}$.

Major progress was achieved in the design and construction of a flow reactor system in series, comprising (1) a continuous stirred tank reactor for performance of the mild hydrotreatment (HT) step; and (2) a tubular reactor for the hydrolytic (BCD) teatment step. The reactor is based on the concept of performing the two depolymerization steps as a single operation. The hydrolytic treatment section of the reactor (2) will allow for use of either methanol or water as a hydrolyzing medium. It 
will also allow for use of either base or acid catalysts.

Significant improvements have been initiated for the Mossbauer laboratory. These include physically moving the laboratory to a better location, renovation of the cryogenic system, installation of a second spectrometer, and design and construction of a high temperature, in situ Mossbauer cell. Good progress has also been made on incorporating a better model for relaxation spectra into the Mossbauer analysis program, and continued analyses of catalysts produced by various investigators in the Consortium. Analyses of the radial structure functions derived from iron $\mathrm{K}$-shell XAFS spectra of $\mathrm{Fe}_{2} \mathrm{O}_{3}$ catalysts show that the apparent coordination numbers of second, third and more distant shells are significantly decreased from those of bulk $\mathrm{Fe}_{2} \mathrm{O}_{3}$, reflecting the ultrafine size of the catalyst particles. In situ Fe K-shell XAFS studies were carried out at temperatures up to $320^{\circ} \mathrm{C}$ in hexahydropyene containing elemental sulfur and either a $30 \AA \mathrm{Fe}_{2} \mathrm{O}_{3}$ catalyst or a sulfated $\mathrm{Fe}_{2} \mathrm{O}_{3}$ catalyst. Rapid transformation to pyrrhotite was observed for the 30 $\AA$ catalyst at $100-180^{\circ} \mathrm{C}$, while the sulfated catalyst, which had a significantly larger particle size, transiormed to pyrrhotite at $250-320^{\circ} \mathrm{C}$.

The reaction of several iron-based catalysts was observed in the transmission electron microscope (TEM) in situ at temperatures up to $600^{\circ} \mathrm{C}$ in $0.3 \mathrm{Torr}$ hydrogen. The catalysts investigated included an $\mathrm{Fe}_{2} \mathrm{O}_{3} / \mathrm{SO}_{4} / 0.5 \% \mathrm{Mo}$ catalyst form the University of Pittsburgh and two $\mathrm{Fe}_{x} \mathrm{~S}_{y}$ catalysts prepared at West Virginia University. The sulfated $\mathrm{Fe}_{2} \mathrm{O}_{3}$ catalyst and one of the WVU catalysts behaved rather similariy. At 150 to $330^{\circ} \mathrm{C}$, a transformation from an initial reedle-like structure to smaller particles $1-5 \mathrm{~nm}$ in diameter occurred. At $330-350^{\circ} \mathrm{C}$, hexagonal reaction pits begin to form all over the graphite, at sites removed from the catalysts. The second $\mathrm{Fe}_{\mathrm{x}} \mathrm{S}_{\mathrm{y}}$ specimen from had a sponge-like appearance and was also observed to transform to particles 2-5 in in diameter at about $210^{\circ} \mathrm{C}$. In this case, square pits were formed which were coated with catalyst.

Electron probe microanalysis (EPMA) has been used to study the dispersion of a bimetallic iron/molybdenum catalyst in demineralized Blind Canyon ccal before and after hydrotreatment. Our EPMA images indicate that before hydrotreatment the impregnation of the coal particles with iron and molybdenum advances no further than the margins of the coal particles regardless of whether impregnation was by the incipient wetness technique or by ultrasonication. After hydrotreatment the EPMA images showed that iron and molybdenum were dispersed fairly uniformly throughout the interior of the coal particles.

These experiments have been repeated with three Argonnt coals: Pittsburgh \#8, Wyodak and Blind Canyon coal. These coals were used as received, i.e. they were not demineralized, and they were impregnated with iron and molybdenum by incipient wetness and by ultrasonication. The EPMA image obtained before and after hydrotreatment of the 
undemineralized Blind Canyon coal were consistent with those obtained previously with demineralized Blind Canyon coal; iron and molybdenum were localized around the border of the coal particles before hydrotreatment and entered the coal particles during hydrotreatment. Results obtained with Pittsburgh \#8 coal closely resemble results obtained with Blind Canyon coal as would be expected since both are high volatility bituminous coals. Wyodak coal shows penetration of iron and molybdenum into the coal particles before hydrotreatment. The higher polarity and porosity of this sub-bituminous coal (than of Blind Canyon and Pittsburgh \#8 coals) may account for coal rank playing a role in catalyst dispersion.

Ferric sulfide $\left(\mathrm{Fe}_{2} \mathrm{~S}_{3}\right)$ has been used as a precursor to synthesize mixtures of pyrite and pyrrhotite. At temperatures greater than $5^{\circ} \mathrm{C}$, ferric sulfide disproportionates to form pyrite $\left(\mathrm{FeS}_{2}\right)$, nonstoichiometric pyrrhotite $\left(\mathrm{FeS}_{\mathrm{x}}\right)$, and elemental sulfur. The kinetics of this reaction has been investigated.

Initial experiments showed that naphthalene hydrogenation to tetralin can be accomplished successfully by the addition of an $\mathrm{Fe}(\mathrm{AcAc})_{3}$ precursor. The activity for hydrogenation of the Fe catalyst generated in situ was greater than the $\mathrm{MoS}_{2}$ generated in situ from Mo naphthenate and excess sulfur. The addition of sulfur to the $\mathrm{Fe}(\mathrm{AcAc})_{3}$ changed the character of the in situ generated $\mathrm{Fe}$ catalyst and reduced its activity for hycirogenation substantially. The amount of sulfur present in the reaction changed the activity of the in situ generated $\mathrm{Fe}$ catalyst, most likely because of a change in stoichiometry.

A new study has been initiated dealing with slurry phase catalysts. These catalysts are being tested in a trickle flow reactor system using a fixed bed of powdered catalyst. The objective is to determine optimum conditions for hydrogenation and cracking reactions. This should provide insight into how iron-based catalysts function and how to pretreat the catalysts for maximum activity and desired selectivity. A pump has been ordered that should allow the reactor to use colloidal catalyst particles in the feed.

An $\mathrm{Fe}_{2} \mathrm{O}_{3} / \mathrm{SO}^{2-}{ }_{4}$ catalyst has been characterized by both SQUID magnetometry and $\mathrm{X}$-ray diffraction line broadening measurements. The resulting catalyst particle size distributions are in reasonable agreement with those reported earlier on the basis of Mossbauer superparamagnetic relaxation times. Additional iron-based catalysts are being investigated by these methods.

A $\mathrm{CO}_{2}$ laser pyrolysis system has been built for the synthesis of ultrafine iron carbide particles by the reaction of $\mathrm{Fe}(\mathrm{CO})_{5}$ and $\mathrm{C}_{2} \mathrm{H}_{4}$. By varying reaction parameters, three pure phase nanosize particles, alpha- $\mathrm{Fe}, \mathrm{Fe}_{3} \mathrm{C}$ and $\mathrm{Fe}_{4} \mathrm{C}_{3}$ with sizes ranging from $6 \mathrm{~nm}$ to $30 \mathrm{~nm}$ have been produced. In particular, $\mathrm{Fe}_{7} \mathrm{C}_{3}$ has been produced for the first time 
in its pure form using this technique. Furthermore, by mixing $\mathrm{H}_{2} \mathrm{~S}$ into the reactant gas stream, nanoscale $(\sim 10 \mathrm{~nm}) \mathrm{Fe}$-sulfide particles identified as pyrrhotite $\left(\mathrm{Fe}_{1-\mathrm{x}} \mathrm{S}, 0<\mathrm{x}<0.125\right)$ have recently been generated. $\mathrm{Fe}_{3} \mathrm{C}_{3}$ gave good liquefaction results when used with DMDS, giving 7-9 percent conversion enhancement (total pyridine solubles) in the 360$390^{\circ} \mathrm{C}$ range.

\section{Novel Coal Liquefaction Concepts}

Sulfolobus brierleyi was used in a fermentation treatment of $\mathrm{KY} \# 11$ coal to promote the formation of $\mathrm{FeOOH}$ and the impregnation of molybdenum on coal particles. The liquefaction yield of the coal was increased by $26 \%$ by this treatment.

Hydrogen uptake hydrogenase-possessing thermoacidophilic archaebacterium Sulfolobus brierleyi and mesophilic sulfate-reducing bacteria Desulfovibrio desulfuricans were used to study the biological hydrogenation of different coals and model compounds. The experiments with model compounds showed hydrogen uptake ranging from 0.28 moles $\mathrm{H}_{2} /$ mole of diphenyl methane to 6.55 moles $\mathrm{H}_{2} /$ mole of 1,2 -diquinolyl ethane in the presence of $S$. brierleyi and $D$. desulfuricans respectively. Previous results for the biological hydrogenation of several coals showed the highest hydrogen uptake for a particular KY \#11 coal sample; liquefaction of this biohydrogenated sample shows an increase of $5.5 \%$ in liquefaction yield.

Methods have been developed for the synthesis of a number of model compounds that will be used in studies of the mechanisms of catalytic coal liquefaction. It has been established that irradiation of methyl 4-cyanobenzoate in the presence of electron donors in $\mathrm{CH}_{3} \mathrm{CN}$ solution will produce desired radical cations of certain model compounds.

The investigation on improving hydrogen transfer in coal liquefaction has focused on the design and construction of a continuous flow reactor system, and on improving the mass transfer contactor design.

Catalytic hydropyrolysis of Blind Canyon coal has been studied using dispersed iron, nickel, or molybdenum in single-element hydropyrolysis. In co-catalyst experiments, iron and nickel were used as promoters for molybdenum. Iron chloride hexahydrate, nickel nitrate hexahydrate and ammonium tetrathiomolybdate were used as precursors. While iron and nickel were relatively ineffective when used alone at relatively high concentrations $(1 \%)$, they improved yields significantly when used as promoters for molybdenum $(0.01 \% \mathrm{Fe}$ or $\mathrm{Ni} / 0.05 \% \mathrm{Mo})$.

Coal liquefaction by solvent extraction accompanied by ultrasonic agitation has 
been studied. The dissolution of coal is clearly improved by application of ultrasonic energy, but the magnitude of improvement depends significantly on the solvent.

\section{Exploratory Research on Coal Conversion}

In the ongoing investigation of coal and coal liquids structure by NMR, new methods of obtaining chemical shift tensors were developed. These methods involve the combination of slow spinning at the magic angle with selective excitation and dipolar dephasing techniques. Preliminary application of these methods to anthracite coals has been made.

It has been established that relaxation times determined by NMR and ENDOR for Utah coal chars are in reasonable agreement.

Excellent progress has been made in setting up a new surface analysis laboratory at one of the CFFLS universities (WVU). Two ultrahigh vacuum surface analysis systems (non-CFFLS funded) were received and appropriate power lines were installed in the lab. Analysis capabilities provided by these systems include Auger electron spectroscopy (AES), electron energy loss spectroscopy (EELS), and low energy electron diffraction (LEED). Both AES and EELS provide information on atomic and molecular composition of the surface and near surface layers, while LEED provides structural information. These systems will also include differentially pumped quadrupole mass spectrometers. Future plans call for the addition of X-ray photoelectron spectroscopy (XPS), scanning tunneling microscopy (STM), and interfaced reaction chambers which will allow the study of even more sophisticated chemical reaction processes.

An on-line GC/MS monitoring system for coal conversion processes under high pressure conditions has been designed and constructed. Typical liquefaction reactions such as hydrogenation, hydrodeoxygenation, hydrodenitrogenation, and hydrodesulfurization have been studied under thermal and catalytic conditions using suitable model compounds. A temperature programmable transfer line GC has been developed for on-line investigation of coal-derived liquids.

An investigation of the catalytic dehydrogenation of model compounds is continuing to delineate the importance of different aspects of catalytic hydrogen transfer reactions. Experiments are currently focused on the catalytic dehydrogenation of 9,10dehydroanthracene using $\mathrm{Pd} / \mathrm{CaCO}_{3}$ and $\mathrm{Pd} /$ carbon catalysts. In the presence of equimolar amounts of 9,10-DHA and stilbene, using several solvents (phenanthridine, quinaldine, and 2-methylnaphthalene), 9,10-DHA was essentially completely dehydrogenated to anthracene with little or no evolution of $\mathrm{H}_{2}$ gas, while the stilbene accepted hydrogen to yield 
bibenzyl. In the absence of a catalyst, little or no reaction occhised.

An electrodynamic balance is being used to investigate the structure and reactions of single coal particles. One initial study has measured adsorption and desorption isotherms of water vapor and helium for single coal particles. Introduction of a focusing system and an optical multichannel analyzer has recently made it possible to rapidly $(\sim 10 s)$ obtain Raman spectra from single coal particles.

The liquefaction pathway of coal-derived asphaltenes has been investigated. The asphaltenes were derived from liquefaction experiments using a Western Kentucky \#9 coal slurried in an Ashland petroleum heavy oil carried out in a one liter stirred autoclave. The asphaltenes obtained from these experiments were liquified in batch microautoclave reactors using a tetralin solvent and a variety of reaction times and temperatures. The primary reaction observed is the conversion of asphaltenes to oils + gases, with the oil solubility class being the primary component of the $\mathrm{O}+\mathrm{G}$.

A new HPLC procedure has been developed for classifying coal liquids that uses three solvents, two types of columns anci a column switching sequence. This sequence separates an oil sample by molecular type and further separates its aromatic molecules by ring size. Initial HPLC studies of coal liquids from iron catalyzed Pittsburgh No. 8 coal showed that the oil fraction contained saturates, polars, and aromatics with ring sizes from 3 to perhaps 8 rings. The asphaltene fraction of the same coal liquid sample contained only polar molecules. 


\section{Task I}

Iron-based Catalysts for Coal Liquefaction

Program Coordinators: Irving Weluder and Gerald P. Huffman 


\title{
Project I.1 Sulfated Iron Oxides as Coal Liquefaction Catalysts
}

\author{
I. Wender and J.W. Tierney \\ University of Pittsburgh
}

\begin{abstract}
SOMMARY
We have made a systematic study of the kinetics of the direct liquefaction of wyodak coal using three finely divided iron oxidebased catalysts: $\mathrm{MO} / \mathrm{Fe}_{2} \mathrm{O}_{3} / \mathrm{SO}_{4}, \mathrm{Fe}_{2} \mathrm{O}_{3} / \mathrm{SO}_{4}$, and $\mathrm{Fe}_{2} \mathrm{O}_{3}$. The objective was to compare the activities of these finely dispersed iron-based catalysts on a quantitative basis. The kinetics of the direct liquefaction of Argonne Wyodak coal ( $\Rightarrow \quad C=75$ ) using tetralin and 1000 psig (cold) $\mathrm{H}_{2}$ were experimentally measured and mathematically modeled. To develop the model, experiments were carried out in a 27 Cc horizontally shake.l autoclave microreactor at $375-425^{\circ} \mathrm{C}$ for reaction times of 0-120 minutes. A simple lumped parameter model using oils, asphaltenes, coal, and gases as the product fractions was used to describe the observed kinetics. Model parameters include freuuency factors and activation energies for four first order reactions, coal to gases, coal to oil, coal to asphaltenes, and asphaltenes to oils. This model successfully describes the effect of catalyst type and catalyst loading and indicates that the asphaltenes to oils reaction is the primary catalytic step. The model successfully predicts the effect of the non-isothermal heatup period in a stirred alutoclave and can be used to predict conversions at temperatures other than those used to determine the model parameters.
\end{abstract}




\section{BACIENT RESULTS}

\section{Yatbematical yodel:}

A kinetic model based on both series and parallel conversion of coal into oils was used as the basis for our analysis. The model is similar to the one employed by Radomyski et al. (1984) who found that a series-parallel model was superior to models based on either only-series or only-parallel pathways. suzuki et al. (1987) have reported that, in a catalytic process, oils are formed via asphaltenes and by decomposition of the organic fraction of coal. A preliminary analysis of our data indicated that only the conversion of asphaltenes to oils was sensitive to the type of the catalyst employed and, therefore, we represent the conversion of asphaltenes to oils by parallel thermal and catalytic patuways as indicated below:

K $c 0$

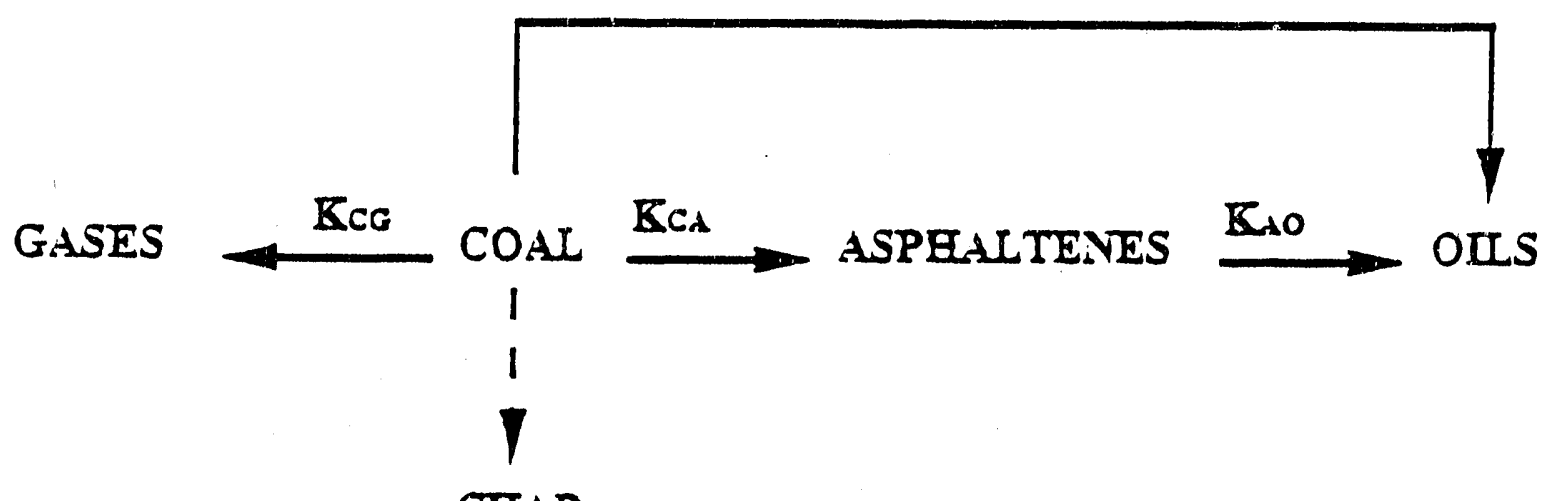

CEAR

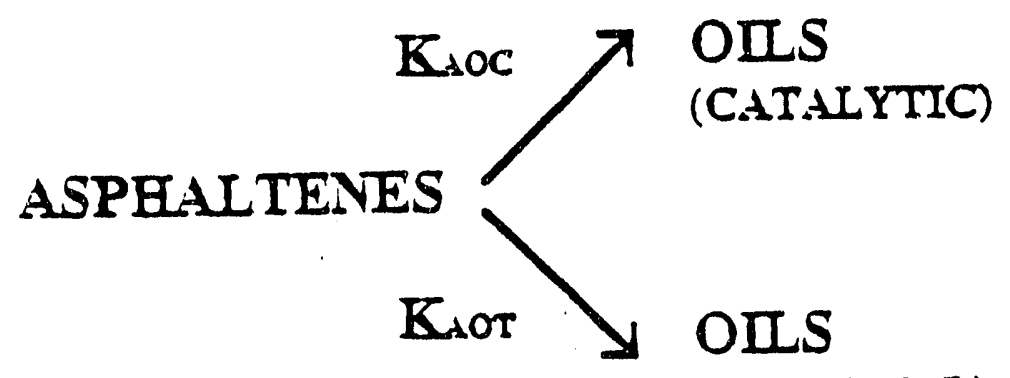

(TEIERMLI) 


\section{Determination of Rinetic Parameters:}

Experimental results were available for 50 runs, 45 made at three temperatures, five reaction times, and three different iron catalysts, plus an additional 5 runs at different weights of catalysts. For each run, the fraction of unconverted coal ( $Y$ ), the asphaltene fraction $\left(Y_{A}\right)$, and the oil fraction $\left(Y_{0}\right)$ were measured. These data were used to calculate best values for the 14 kinetic parameters (seven pre-exponential factors and seven activation energies) by minimizing the following function:

$$
f=\frac{\sum\left[W_{C}\left(Y_{\theta, 1}-Y_{p_{1,1}}\right)^{2}+W_{A} 1\left(Y_{A_{0,1}}-Y_{A_{p, 1}}\right)^{2}+W_{O}\left(Y_{O_{0,1}}-Y_{O_{p, 1}}\right)^{2}\right]}{N}
$$

where, $f=$ objective function to be minimized; $Y, Y_{A}$, and $Y_{0}$ represent coal, asphaltenes, and oils respectively. The $W^{\prime} s$ are their corresponding weighting factors and the subscripts $e$ and $p$ stand for the experimental and the predicted values.

A quasi-Newtonian minimization procedure was used to minimize the mean squared error function defined by $f$ in equation (1). The weighting factors ( $W^{\prime} S$ ) for product fractions were based on the reproducibility in duplicate runs and were $1,0.9$, and 0.8 respectively. Since the conversions to gases in our experimental work-up were determined by difference and include all the handing losses, this fraction was not included in the correlation.

The mean squared error function, $f$, was minimized using an IMSL (International Mathematical Subroutines Library) subroutine, ZXMIN, which minimizes a function of $n$ variables using a quasiNewtonian Method. We were able to obtain values of the frequency factors and the apparent activation energies for the seven steps in our model (four thermal and three catalytic). All 50 data points were used to minimize $f$. The values of the model parameters (frequency factors and activation energies) are listed in Table 1. Reaction rate constants are shown in Table 2 for thermal reactions and in Table 3 for catalytic reactions. 
Table 1. Activation Energies and rrequency factors for rinetio Kodel

\begin{tabular}{|c|c|c|}
\hline Model step & $\begin{array}{c}\text { Activation } \\
\text { Energy } \\
\mathrm{kcal} / \mathrm{gmole}\end{array}$ & $\underset{\min ^{-1}}{\text { Frequetor }}$ \\
\hline c $-\cdots$ A (Thermal) & 13.20 & 646.70 \\
\hline c ---> o (Thermal) & 5.58 & 2.21 \\
\hline$c \leadsto-\rightarrow G$ (Thermal) & 23.60 & 442800 \\
\hline A $\rightarrow-\infty 0$ (Thermal) & 67.86 & 760.30 \\
\hline$A-\cdots 0$ (Catalyst I) & 5.21 & 7.21 \\
\hline A $-\rightarrow 0$ (Catalyst II) & 15.11 & 73320 \\
\hline A $--\rightarrow 0$ (Catalyst III) & 13.50 & 43080 \\
\hline
\end{tabular}

Table 2. Rate Constants for Thermal stops in Model

\begin{tabular}{|l|c|c|c|}
\hline \multirow{2}{*}{$\begin{array}{c}\text { Rate Constant } \\
\left(\mathrm{min}^{-1}\right)\end{array}$} & $375^{\circ} \mathrm{C}$ & $100^{\circ} \mathrm{C}$ & $125^{\circ} \mathrm{C}$ \\
\cline { 2 - 4 } & $2.30 \mathrm{E}-2$ & $3.40 \mathrm{E}-2$ & $4.83 \mathrm{E}-2$ \\
\hline$K_{\mathrm{CA}}$ & $1.0 \mathrm{E}-10$ & $2.1 \mathrm{E}-10$ & $4.1 \mathrm{E}-10$ \\
\hline$K_{\mathrm{AOT}}$ & $2.89 \mathrm{E}-2$ & $3.40 \mathrm{E}-2$ & $3.95 \mathrm{E}-2$ \\
\hline$K_{\mathrm{CO}}$ & $4.70 \mathrm{E}-3$ & $9.40 \mathrm{E}-3$ & $1.76 \mathrm{E}-2$ \\
\hline$K_{\mathrm{CO}}$ & \multicolumn{3}{|c|}{ TEMPERATURI } \\
\hline
\end{tabular}

\section{Effect of Temperature and Type of Catalyst Fuployed:}

One of the main objectives of this study was to obtain quantitative measures of the effectiveness of the three catalytic systems at 375,400 , and $425^{\circ} \mathrm{C}$. It can be seen, first of all, that the thermal rate constants in Table $2, \mathrm{~K}_{\mathrm{AOT}}$, which represent conversion of asphaltenes to oils by thermal pathways are 
Table 3. Rate Constants for Catalytic steps in Model

\begin{tabular}{||c|c|c|c|}
\hline Catalyst system & \multicolumn{3}{|c|}{ TEMPERATURE } \\
\cline { 2 - 4 } & $375^{\circ} \mathrm{C}$ & $100^{\circ} \mathrm{C}$ & $125^{\circ} \mathrm{C}$ \\
\hline $\mathrm{Fe}_{2} \mathrm{O}_{3}+8$ & $1.26 \mathrm{E}-3$ & $1.46 \mathrm{E}-3$ & $1.68 \mathrm{E}-3$ \\
\hline $\mathrm{Fe}_{2} \mathrm{O}_{3} / 8 \mathrm{O}_{4}+8$ & $5.87 \mathrm{E}-3$ & $9.08 \mathrm{E}-3$ & $1.36 \mathrm{E}-2$ \\
\hline $\mathrm{MO} / \mathrm{Fe}_{2} \mathrm{O}_{3} / 8 \mathrm{O}_{4}+\mathrm{s}$ & $1.20 \mathrm{E}-2$ & $1.77 \mathrm{E}-2$ & $2.55 \mathrm{E}-2$ \\
\hline
\end{tabular}

negligible compared to the catalytic rate constants for the same steps as shown in Table 3. Furthermore, the catalytic rate constants (Table 3 ) increase by almost an order of magnitude on going from an unsulfated iron oxide catalyst, $k_{A O C I}$ to the molybdenum-containing sulfated iron oxide catalyst, $\mathrm{K}_{\mathrm{A} O \mathrm{~cm}}$. This indicates that the rate of conversion of intermediate asphaltenes to oils is catalytic and depends strongly on the amount and nature of the catalytic system employed. The conversions to light oils (n-pentane solubles) obtained at 375,400 and $425^{\circ} \mathrm{C}$ using the three catalytic systems are shown as a function of time in Figure 1 .

That the conversion of intermediate asphaltenes to lighter oils is predominantly catalytic has been reported by suzuki et al. (1987) for the direct liquefaction of low-rank and bituminous coals using soluble organometallic complexes as catalysts.

\section{Efect of Catalyst Loading:}

In order to assess the dependence of the catalytic rate constants on the amount of catalyst, experiments were carried out at reduced catalyst concentrations using the $\mathrm{Fe}_{2} \mathrm{O}_{3} / \mathrm{SO}_{4}+\mathrm{S}$ catalytic system at $400^{\circ} \mathrm{C}$. All of the catalytic experiments discussed heretofore in our kinetic studies were carried out using $3500 \mathrm{ppm}$ Fe based upon the coal charge. To study the effect of reduced catalyst concentrations, the concentration of iron was reduced to $1750 \mathrm{ppm}$ with respect to coal. The observed and predicted product fractions for 3500 and $1750 \mathrm{ppm}$ Fe loading are shown in Figures 2 and 3 as a function of time. 


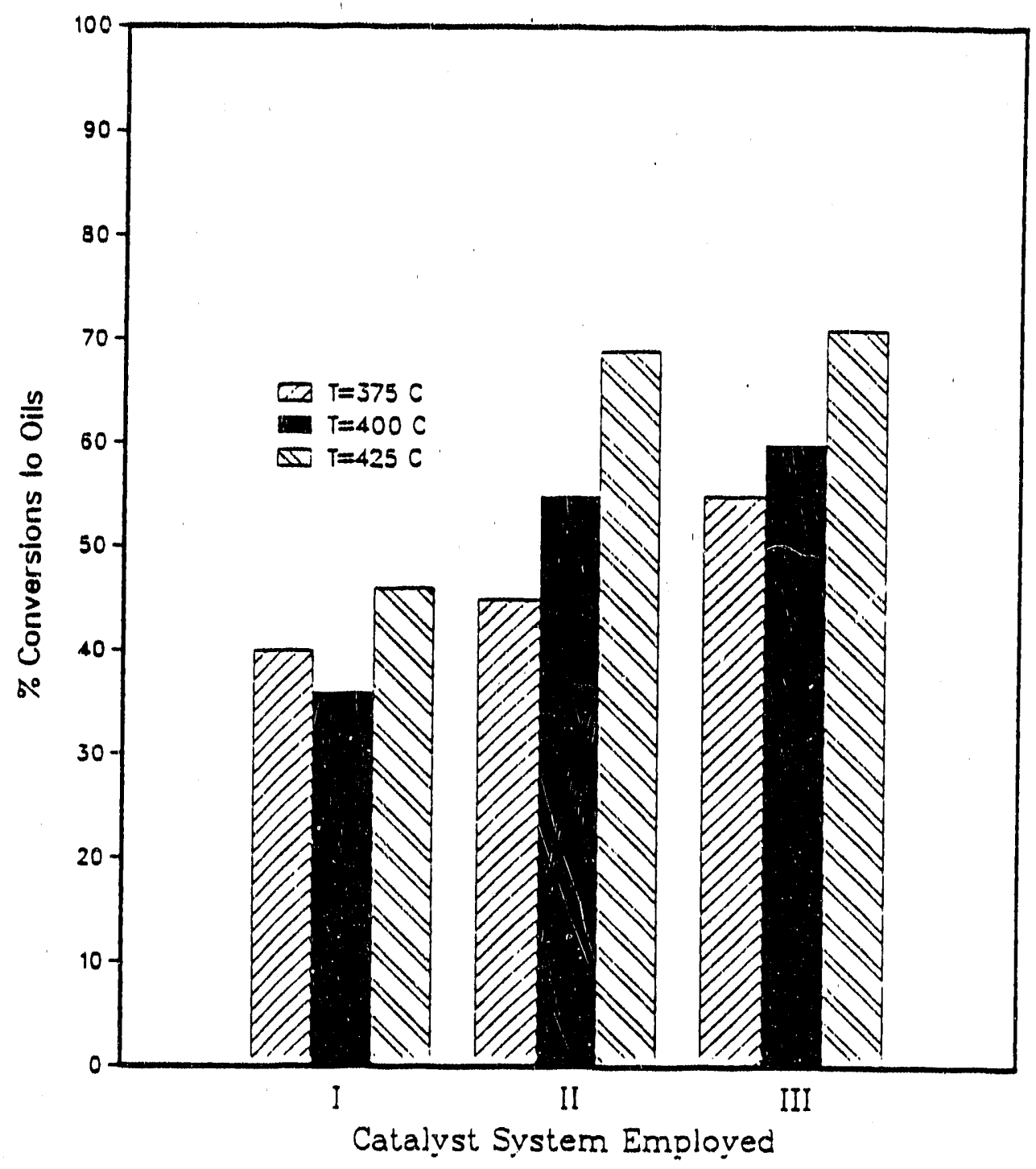

Pigure.1.Conversions to oils at Three Temperatures Using Three Different Catalysts 


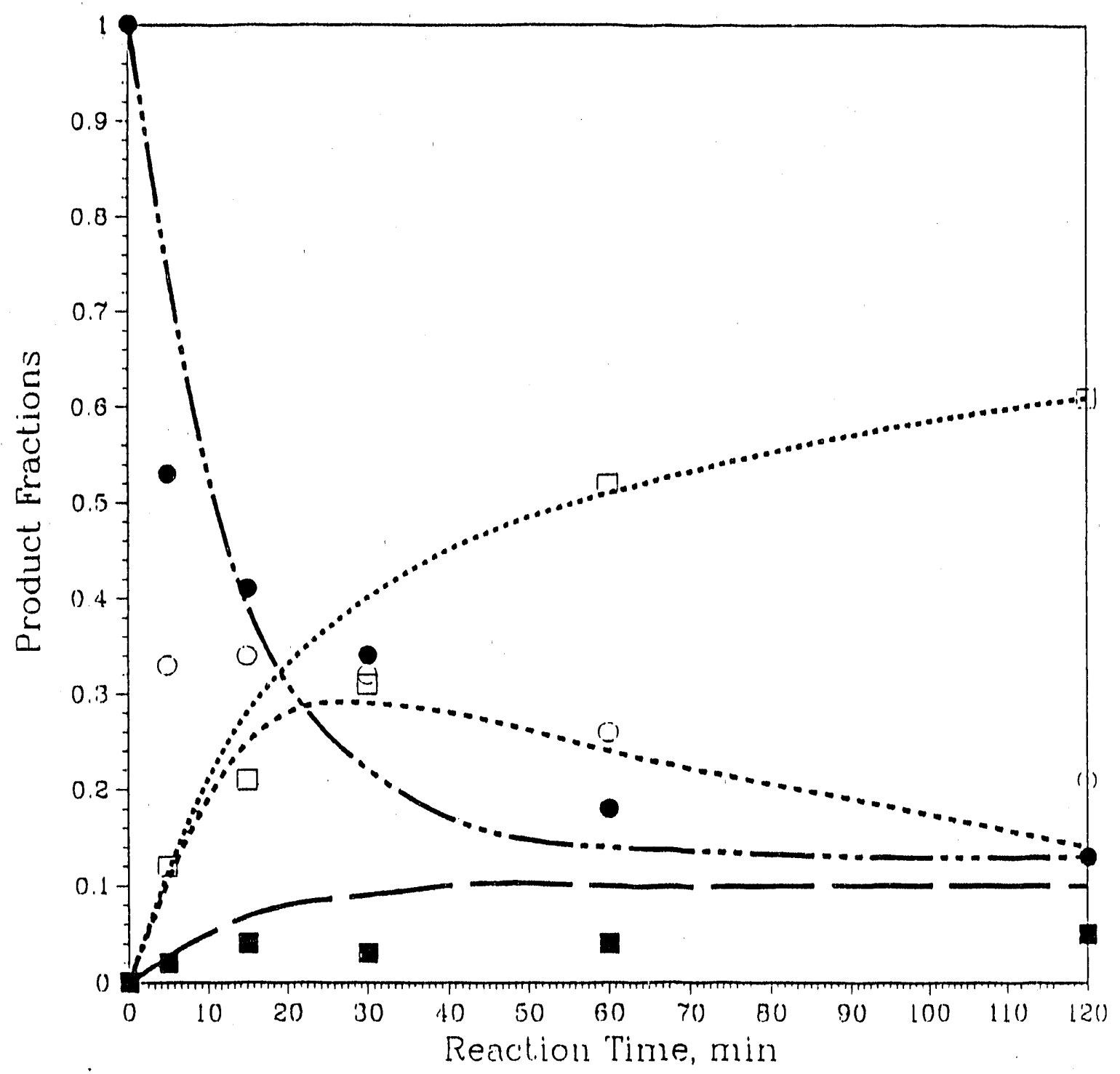

Figure 2. Predicted versus observed Product Fractions for the sulfated Iron oxide Catalyst (3500 ppm Fe) as a Function of Time.' 


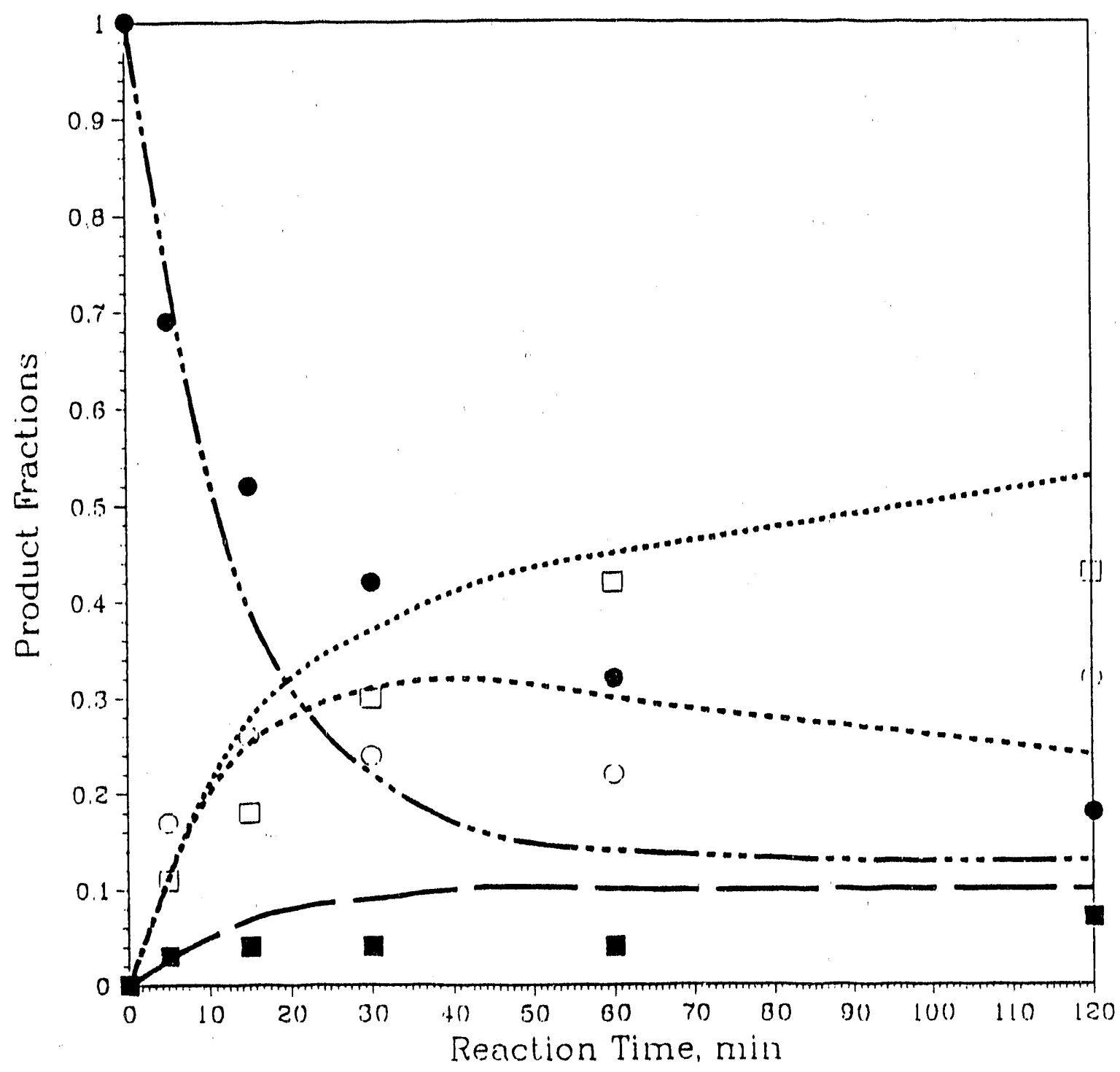

Figure 3. Predicted versus observed product Fractions for sulfated Iron Oxide Catalyst $(1750 \mathrm{ppm} B \theta)$ as a Function of Time. 


\section{Mode1 Applications:}

To check extrapolations using the model, we carried out reactions at temperatures of 300 and $350^{\circ} \mathrm{C}$ for a half hour of reaction time and measured conversions and product distributions. These were also calculated using the model equations. Observed and predicted product fractions are shown in Table 4 . At $350^{\circ} \mathrm{C}$, the predicted product fractions were within 3 percent of experimental, which is very good. At $300^{\circ} \mathrm{C}$, agreement is poorer but the extrapolation is much greater $\left(75^{\circ} \mathrm{C}\right)$. The limiting coal conversion (C*) as a function of temperature is needed for the model. Values were available at 375,400 , and $425^{\circ} \mathrm{C}$ as discussed above. Extrapolation to the lower temperatures was done by fitting these points to an exponential equation.

$$
C^{*}=1-812 \exp (-0.0128 \mathrm{~T})
$$

\section{Table 4. Comparison of Model Predictions and Experimental} Values of Product Fractions for Low Temperature Runs.

\begin{tabular}{|c|c|c|c|c|}
\hline \multicolumn{5}{|c|}{$\begin{array}{l}\text { Reaction Conditions: Wyodak coal, } 30 \mathrm{~min}, 1000 \mathrm{psig} \text { (cold) } \\
\mathrm{H}_{2}, 160 \mathrm{cpm} \text { shaker speed, Catalyst } \mathrm{II} \text {, ratio } \mathrm{Fe} / \mathrm{Coal}=3500 \\
\text { ppm, tetralin/coal= } 4: 1 \text {. }\end{array}$} \\
\hline Temp. ${ }^{\circ} \mathrm{C}$ & Coal & Asphaltanes & O11s & Gases \\
\hline \multicolumn{5}{|l|}{300} \\
\hline Model & 0.76 & 0.06 & 0.17 & 0.01 \\
\hline Experiment & 0.68 & 0.12 & 0.15 & 0.05 \\
\hline \multicolumn{5}{|l|}{350} \\
\hline Model & 0.49 & 0.17 & 0.30 & 0.04 \\
\hline Experiment & 0.52 & 0.15 & 0.26 & 0.07 \\
\hline
\end{tabular}

Another test of the model was made by predicting product fractions for the three non-catalytic (thermal) reactions carried out to determine ultimate total coal conversions. The results are 
Table 5. Comparison of Model Pxediotions and Experimental Product Practions for Non-Catalytio Runs

\begin{tabular}{|c|c|c|c|c|}
\hline \multicolumn{5}{|c|}{$\begin{array}{l}\text { Reaction Conditions: myodak Coal, } 180 \mathrm{~min}, 1000 \mathrm{psig}\left(\text { cold) } \mathrm{H}_{2}\right. \\
160 \mathrm{cpm} \text { shaker speed, tetral in/coal ratio 4:1, no catalyst. }\end{array}$} \\
\hline remp. ${ }^{\circ} \mathrm{C}$ & $\operatorname{con} 1$ & Asphaltones & 011 s & Gases \\
\hline \multicolumn{5}{|l|}{375} \\
\hline Model & 0.21 & 0.33 & 0.40 & 0.06 \\
\hline Experiment & 0.24 & 0.36 & 0.35 & 0.05 \\
\hline \multicolumn{5}{|l|}{100} \\
\hline Model & 0.15 & 0.37 & 0.38 & 0.10 \\
\hline Experiment & 0.13 & 0.41 & 0.40 & 0.06 \\
\hline \multicolumn{5}{|l|}{125} \\
\hline Model & 0.10 & 0.42 & 0.34 & 0.14 \\
\hline Experiment & 0.07 & 0.43 & 0.44 & 0.06 \\
\hline
\end{tabular}

shown in Table 5 and indicate that the model successfully accounts for the thermal as well as the catalytic reactions. There is a substantial yield of olls in the absence of catalysts, indicating that the conversion to oils is not through intermediate asphaltenes. The primary catalytic effect is in the conversion of asphaltenes to oils.

\section{POTORE WORK}

(i) Catalyst concentration vs. conversion studies will be carried out for the direct liquefaction of a low-pyrite bituminous coal with tetralin as a solvent (4:1 solvent-to-coal) in the presence of sulfated oxide/hydrous oxide catalysts.

(i1) Model compounds reactions will also be carried out to elucidate the mode of action of the sulfated metal oxide catalysts.

(iii) Analysis of the coal liquefaction reaction products will be carried out using GC, GC-MS, NMR, and vacuum distillation. 


\section{REFERENCES}

1. Radomyski, B. and J. Szczygiel, Fuel, Vol. 63, 1984.

2. Suzuki, T.; Ando, T.; Y. Watanabe, Energy \& Fuels, Vol.1, No. 3,1987 . 


\title{
Project 1.2 Low Temperature Depolymerization and Liquefaction of Premium U.S. Coal Samples
}

\author{
J.S. Shabtai, K.V.R. Chary and J. Hu \\ University of Utah
}

The main research activities under this project during the August 1 - October 31, 1991 period were as follows:

1. Continuation of the systematic studies on a premium Lewiston-Stockton (W.V.) coal with the purpose of determining suitable $\mathrm{HT}-\mathrm{BCD}$ processing conditions for high-yield conversion of this feed into depolymerized products. Table 1 summarizes some of the relevant results obtained. As seen, for a selected set of conditions in the initial HT step (reaction temperature, $275^{\circ} \mathrm{C} ; \mathrm{H}_{2}$ pressure, $1500 \mathrm{psig}$; reaction time, $1 \mathrm{~h}$ ), the total yield of depolymerized (THF-soluble) products in the subsequent BCD step increases markedly with increase in $\mathrm{BCD}$ temperature (from $37.1 \mathrm{wt} \%$ at $250^{\circ} \mathrm{C}$ to $85.9 \mathrm{wt} \%$ at $305^{\circ} \mathrm{C}$ ). Furthermore, the proportion of the oil fraction in the solubilized product increases from $54.3 \mathrm{wt} \%$ at $250^{\circ} \mathrm{C}$ to $71.7 \mathrm{wt} \%$ at $305^{\circ} \mathrm{C}$. It should be noted that the oil fraction consists mainly of cyclohexane-solubles together with a small amount of toluene-solubles. GC/MS analysis of the latter showed that those are predominantly monocluster compounds having molecular weights similar to those of the cyclohexanesolubles (M.W. in the approximate range of 100-280) and differing only in somewhat higher oxygen content (higher concentration of O-containing groups) which results in lower solubility in cyclohexane. For this reason, the cyclohexane-solubles and the small amount of toluene-solubles are reported in Table 1 as a single oil fraction.

In another part of the study the hydrogenolytic activity of the $\mathrm{Fe}\left(\mathrm{H}_{2} \mathrm{O}\right)_{6} \mathrm{Cl}_{3}$ catalyst in the HT step was compared with that of anhydrous $\mathrm{FeCl}_{3}$. Comparative runs were performed with Lewiston-Stockton coal as feed, using equivalent amounts (in terms of $\mathrm{Fe}$ content) of the impregnated salts. The HT temperature range was from $250-305^{\circ} \mathrm{C}$. An identical $\mathrm{H}_{2}$ pressure of $1,500 \mathrm{psig}$ and a reaction time of $1 \mathrm{~h}$ were kept in all runs. The total yields of THF-soluble products (including some acetone-solubles) obtained in the HT step with the two types of catalysts were as follows:

\begin{tabular}{ccc}
\hline Reaction Temp, C & $\mathrm{Fe}\left(\mathrm{H}_{2}\right)_{6} \mathrm{CL}_{3}$ Catalyst & anh. $\mathrm{FeCl}_{3}$ Catalyst \\
\hline 250 & 7.2 & 5.6 \\
275 & 15.3 & 9.7 \\
290 & 18.5 & 12.6 \\
305 & 26.7 & 15.9 \\
\hline
\end{tabular}


The results demonstrate the markedly higher hydrogenolytic activity of the $\mathrm{Fe}\left(\mathrm{H}_{2} \mathrm{O}_{6}{ }^{\mathrm{B}}+\right.$ ion as compared with that of the anhydrous $\mathrm{Fe}^{3+}{ }_{6}$ ion. This is in agreement with results obtained in model compound studies using diphenylmethane as feed (see preceding Progress Report for the period May 1-June 30, 1991). The higher activity of the aqua complex can be ascribed to its transformation into a strong protonic acid at temperatures above $250^{\circ} \mathrm{C}$.

2. Continuation of model compound studies with solid Fe-containing catalysts.

A detailed kinetic study of the hydrogenolysis reactions of 4-benzylbiphenyl in the presence of a $\mathrm{FeCl}_{3} / \mathrm{SiO}_{2}-\mathrm{Al}_{2} \mathrm{O}_{3}$ catalyst was continued. This compound contains three differint hydrogenolysis-susceptible types of linkages, characterized by different bond dissociation energies and different steric environments. The rates of the three competing cleavage reactions are being determined and the kinetic framework of the overall process is being elucidated. A complete summary of the data and a discussion of the reaction mechanism will be included in the next Progress Report. Studies with other Fe-containing solid catalysts were initiated.

3. Construction of a flow reactor system for the unified HT-BCD coal depolymerization procedure.

Major progress was achieved in the design and construction of a flow reactor system in series, comprising (1) a continuous stirred tank reactor for performance of the mild hydrotreatment (HT) step; and (2) a tubular reactor for the hydrolytic (BCD) treatment step. The reactor is based on the concept of performing the two depolymerization steps as a single operation. The hydrolytic treatment section of the reactor (2) will allow for use of either methanol or water as a hydrolyzing medium. It will also allow for use of either base or acid catalysts. More detailed data will be provided in the next Progress Report.

\section{Table 1}

Yields and Distribution of Depolymerized Products from HT-BCD Treatment of Lewiston-Stockton (WV) Coal, as a Function of BCD Temperature

\begin{tabular}{|c|c|c|c|c|}
\hline \multirow[b]{2}{*}{ Run No. } & \multirow[b]{2}{*}{$\begin{array}{c}\mathrm{BCD}^{\wedge} \\
\text { temp., } \mathrm{C}\end{array}$} & \multirow[b]{2}{*}{$\begin{array}{c}\text { Total } \\
\text { yield, wt\% }\end{array}$} & \multicolumn{2}{|c|}{ Product distribution } \\
\hline & & & $\begin{array}{l}\text { Oil } \\
\text { fr.c }\end{array}$ & $\begin{array}{l}\text { Incompletely } \\
\text { depol. fr.d }\end{array}$ \\
\hline $\mathrm{CH}-\mathrm{I}$ & 250 & 37.1 & 54.3 & 45.7 \\
\hline $\mathrm{CH}-2$ & 275 & 53.7 & 63.5 & 36.5 \\
\hline $\mathrm{CH}-3$ & 290 & 76.0 & 67.0 & 33.0 \\
\hline $\mathrm{CH}-4$ & 305 & 85.9 & 71.7 & 28.3 \\
\hline
\end{tabular}


"The conditions used in the preceding HT step were as follows: temperature, $275^{\circ} \mathrm{C}$; initial $\mathrm{H}_{2}$ pressure, 1500 psig; reaction time, $1 \mathrm{~h}$.

'Total yield of THF-soluble products, excluding $10.2 \mathrm{wt} \%$ of THF-soluble pre-extract.

${ }^{\circ}$ Cyclohexane-solubles, plus a small amount of toluene-solubles (see text).

'THF-solubles (toluene-insolubles). 


\title{
Project I.3 Generic Structural Characterization and Liquefaction Research
}

\author{
G.P. Huffrnan, N. Shah, J. Zhao, B. Ganguly, K.R.P.M. Rao, \\ M. Mehdi Taghiei, and F.E. Huggins
}

\section{INTRODUCTION}

This report summarizes research activities in the period May 1, 1991 through October 31, 1991. Significant items are as follows:

(1) Papers on the structure and dispersion of iron-based catalysts were presented at and published in the proceedings of the International Conference on Coal Science[1] in Newcastle, Engiand and the Pittsburgh Coal Conference[2]. An oral presentation and three poster presentations were also made at the Fifth Annual CFFLS Technical Meeting, held in Lexington in August. Additionally, characterization results for sulfated $\mathrm{Fe}_{2} \mathrm{O}_{3}$ catalysts were published in a recent article in Energy \& Fuels [3].

(2) To increase the efficiency of our Mossbauer catalyst characterization activity for the CFFLS community, several improvements in the Mossbauer laboratory have been made or initiated: these include moving the laboratory to a new more spacious and vibration-free location, purchase and installation of a second Mossbauer spectrometer, renovation of the Mossbauer cryogenic system, and construction of a new high temperature cell for in situ Mossbauer studies.

(3) A number of new catalysts prepared by various investigators in the CFFLS have been characterized by Mossbaue: and XAFS spectroscopy.

(4) In situ XAFS studies have been carried out on $\mathrm{Fe}_{2} \mathrm{O}_{3}$ catalysts in hexahydropyrene (HHP) plus elemental sulfur at temperatures up to $320^{\circ} \mathrm{C}$.

The results of this work are briefly summarized below.

\section{XAFS SPECTROSCOPY RESULTS}

As discussed in previous reports and publications [1,2,7], Fourier transformation of the extended $x$-ray absorption fine structure (EXAFS) portion of the XAFS spectra yields a radial 
structure function that is similar to a radial distribution function. That is, each peak in the RSF represents the photoelectron scattering component from a shell of neighboring atoms surrounding the iron atom at the origin. This is shown clearly in Figure 1 where the RSF is compared to the true radial distribution function of bulk $\mathrm{Fe}_{2} \mathrm{O}_{3}$; the RSF has been shifted forward slightly in Fig. 1 to account for the effects of phase shifts [4].

In Figure 2, the RSF of bulk $\mathrm{Fe}_{2} \mathrm{O}_{3}$ is compared to that of several catalysts; a $30 \mathrm{~A}$ $\mathrm{Fe}_{2} \mathrm{O}_{3}$ sample prepared by a company called Mach $\mathrm{I}$, and two sulfated $\mathrm{Fe}_{2} \mathrm{O}_{3}$ samples prepared by the CFFLS group at the University of Pittshurgh. It is seen that the amplitudes of the iron shell peaks of the catalysts are significantly smaller than those of the bulk standard. By performing a least squares analysis of the back transform of a peak or set of peaks in the RSF, it is possible to determine both the interatomic distances and coordination numbers of the neighbor shells in question [4,5]. The results of such analyses for the catalysts of Figure 2 are presented in Table 1. It is seen that while the interatomic distances are within the standard deviations of those of bulk hematite for the most part, the coordination numbers of the iron shells are markedly decreased from those of bulk hematite. This reflects that fact that the iron atoms on or near the surfaces of these ultrafine catalyst particles have on average less iron neighbors in these shells. Similar results have been observed for catalysts that assume the $\mathrm{FeOOH}$ structure in the as-prepared or as-dispersed state, such as those prepared by cation-exchange or by the Shabtai treatment.

Greegor and Lytle have observed similar results in their EXAFS studies of supported metal catalysts [5] and have developed a model relating average coordination numbers for different neighbor shells to the size and shape of the catalyst particles. We have developed a similar model to do the same thing with catalysts of the $\mathrm{Fe}_{2} \mathrm{O}_{3}$ structure. However, the catalyst particle diameters we have derived from this model are somewhat smaller that expected. For the catalysts of Table 1, we obtain the following results: $\mathrm{Fe}_{2} \mathrm{O}_{3} / \mathrm{SO}_{4}(1991) \sim 20$ $\AA ; \mathrm{Fe}_{2} \mathrm{O}_{3} / \mathrm{SO}_{4}(1990) \sim 15 \AA ;$ and $\mathrm{Fe}_{2} \mathrm{O}_{3}$ (30 $\AA$ sample) $<10 \AA$. The origin of this size underestimation is believed to arise from vibrational and electron mean path effects. This will be explored further. 
In this research period, we have also initiated new in situ studies of catalyst reactions and transformations in a hydrogen donor solvent, hexahydropyene (HHP), at $100-320^{\circ} \mathrm{C}$ in the presence of sulfur. The radial structure functions (RSF) obtained from these studies for the $30 \AA \mathrm{Fe}_{2} \mathrm{O}_{3}$ catalyst and for a sulfated $\mathrm{Fe}_{2} \mathrm{O}_{3}$ catalyst are shown in Figures 3 and 4. Peaks derived from oxygen, sulfur and iron shells are labeled. The results are summarized in Table 2, which gives the interatomic distances and coordination numbers for various neightoring atomic shells to the iron atoms in $\mathrm{Fe}_{2} \mathrm{O}_{3}$ and $\mathrm{Fe}_{(1-x)} \mathrm{S}$, and the same information derived from the RSF of the catalysts by back transform analysis. It is seen that transformation of the 30 $\AA \mathrm{Fe}_{2} \mathrm{O}_{3}$ catalyst to pyrrhotite begins at as low a temperature as $110 \mathrm{C}$, is accelerated at $180^{\circ} \mathrm{C}$, and is completed fairly rapidly at $320^{\circ} \mathrm{C}$. The sulfated $\mathrm{Fe}_{2} \mathrm{O}_{3}$ catalyst, which is much larger in mean particle size $(>100 \AA)$, does exhibit any transformation below $250^{\circ} \mathrm{C}$, and requires some time (several XAFS scans of about 45 minutes each) to completely transform to pyrrhotite at $320^{\circ} \mathrm{C}$.

In the next quarter, these data will be analyzed more completely. Additionally, in situ sulfur K-edge spectra will be investigated, and additional iron K-edge spectra will be obtained. 9,10 - dihydrophenanthrene (DHP) will also be utilized as the hydrogen donor in some experiments, and different heating schedules will be tried. By conducting these experiments in conjunction with reactivity studies at PETC, it should be possible to arrive at more accurate catalyst reactivity-structure relationships.

\section{MOSSBAUER SPECTROSCOPY RESULTS}

\section{III.1 Laboratory Renovations}

Because of heavy demand for Mossbauer spectroscopy analyses, it was decided to completely renovate the Mossbauer laboratory to improve efficiency. First, occasional problems with vibration and an ongoing problem with space were alleviated by moving the laboratory to a new, vibration-free, ground floor location in another building. Second, it was decided to renovate the Mossbauer cryogenic system because significant running time was being lost due to vacuum problems, poor heater and temperature control performance, and general unreliability of the system. The renovations on the low-temperature system included 
installation of a new digital temperature controller and heating element, repair of several small leaks in the helium refrigerant system, and purchase of a new turbomolecular vacuum pump. We are currently awaiting delivery of the vacuum pump after which low temperature Mossbauer studies will resume.

It was also decided to bring a second Mossbauer spectrometer on line. Many of the items required to do this (detectors, multichannel analyzer, amplifiers, etc.) were already on hand. However, it was decided to purchase a new Mossbauer drive unit from a German firm (Hankel) rather than build one. This drive has arrived and all necessary mounting apparatus has been built in the UK machine shops. We are currently awaiting delivery of a power supply and a new radioactive Mossbauer source to make this spectrometer operational.

Finally, we have designed and constructed a new high temperature cell for in situ Mossbauer studies of catalyst reactions. The design should allow catalyst investigation in a hydrogen donor or other solvent, under hydrogen and or hydrogen sulfide gases, at temperatures up to $600^{\circ} \mathrm{C}$. We plan to test this system in the next quarter.

\section{II.2 New Mossbauer Spectroscopy Results}

In previous work, Mossbauer spectroscopy has been used to determine approximate size distributions of ultrafine particle ferric oxide catalysts. Such catalysts exhibit supermagnetic spectra, as illustrated by Figure 5. Both hematite $\left(\alpha-\mathrm{Fe}_{2} \mathrm{O}_{3}\right)$ and the most common iron oxyhydroxide, goethite $(\alpha-\mathrm{FeOOH})$, are antiferromagnetic and would normally exhibit only six-line magnetic hyperfine spectra at room temperature and below. However, as seen in Figure 5, the catalyst samples exhibit a significant quadrupole component. Such behavior is typical of superparamagnetic systems[1-3,6,7].

By use of a simple semiclassical approximation, it is possible to use such superparamagnetic(SP) behavior to derive approximate sizes of the catalyst particles. As discussed elsewhere $[1-3,6,7]$, a critical volume, $V_{e}$, can be defined by 


$$
V_{c}=\underset{K_{a}}{k T} \ln \left(f_{0} / f_{L}\right)
$$

where $\mathrm{T}$ is the absolute temperature, $\mathrm{k}$ is the Boltzmann constant, $\mathrm{K}$ is the magnetic anisotropy constant $f_{O}$ is a constant frequency determined by the material properties, and $f_{L}$ is the Larmor precession frequency of the ${ }^{57} \mathrm{Fe}$ nucleus. If it is assumed that particles exceeding the critical volume give rise to magnetic hyperfine spectra, while particles smaller than that volume exhibit quadrupole spectra, the Mossbauer spectra can be used to derive particle size information. For example, the spectra of Figure 5 indicate that all of the particles in the $\mathrm{FeOOH} / \mathrm{SO}_{4}$ catalyst are smaller than $65 \AA$ in diameter, assuming spherical particles. Similarly, under the same assumption, the two $\mathrm{Fe}_{2} \mathrm{O}_{3} / \mathrm{SO}_{4}$ samples of Figure 1 contain $60(40) \%$ and $2(98) \%$ of particles less (greater) than $85 \AA$ in diameter.

As discussed in our previous report, a model based on the Gutowsky, McCall, and Slichter (GMS) equations $[7,8]$ is being used to generate relaxation spectra of Fe oxides. According to this model, the hyperfine field is usually generated by the polarizing effects of unpaired electron spins. The spin direction can alter or "flip" after a period of time by one of the several mechanisms; this is relaxation phenomenon. The two main processes that cause spin flipping are electronic spin-spin interactions and spin-lattice interactions. In this model only spin-spin relaxation is considered.

Using this model, simulated spectra for different values of relaxation time can be generated. A fitting routine is being developed to fit the experimental data to these simulated spectra for a weighted distribution of relaxation times. The relaxation times and weightages will be the variables to be fitted. Once this is completed, it may be possible to determine a particle size distribution (which is related to relaxation time) from the Mossbauer spectra taken at a single temperature only, if a functional shape (such as a log normal distribution) can be assumed for the particle size distribution. 
Finally, Mossbauer spectra have been obtained from catalysts from several CFFLS investigators at room temperature. We will report these results in the next report after the low temperature data are completed.

\section{SUMMARY}

In the current reporting period, significant improvements have initiated for the Mossbauer laboratory. These include physically moving the laboratory to a better location, renovation of the cryogenic system, installation of a second spectrometer, and design and construction of a high temperature, in situ Mossbauer cell. Good progress has also been made on incorporating a better model for relaxation spectra into the Mossbauer analysis program, and continued analyses of catalysts produced by various investigators in the Consortium. Analyses of the radial structure functions derived from iron K-shell XAFS spectra of $\mathrm{Fe}_{2} \mathrm{O}_{3}$ catalysts show that the apparent coordination numbers of second, third and more distant shells are significantly decreased from those of bulk $\mathrm{Fe}_{2} \mathrm{O}_{3}$, reflecting the ultrafine size of the catalyst particles. In situ Fe K-shell XAFS studies were carried out at temperatures up to $320^{\circ} \mathrm{C}$ in hexahydropyene containing elemental sulfur and either a $30 \AA$ $\mathrm{Fe}_{2} \mathrm{O}_{3}$ catalyst or a sulfated $\mathrm{Fe}_{2} \mathrm{O}_{3}$ catalyst. Rapid transformation to pyrrhotite was observed for the $30 \AA$ catalyst at $100-180^{\circ} \mathrm{C}$, while the sulfated catalyst, which had a significantly larger particle size, transformed to pyrrhotite at $250-320^{\circ} \mathrm{C}$.

\section{REFERENCES}

1. Huffman, G.P.; Huggins, F.E.; Ganguly, B.; Shah, N.; Taghiei, M.M.; Hager, G.T.; Structure and Dispersion of Iron-Based Liquefaction Catalysts", Proceedings, 1991 International Conference on Coal Science, Newcastle, England, Int. Energy Agency, Butterworth-Heineman, Ltd., 1991, 826-829.

2. Huffman, G.P.; Huggins, F.E.; Seehra, M.S.; Baker, R.T.K.; Eyring, E.M.; and Reucroft, P.A.; Proceedings, Eighth Annual Pittsburgh Coal Conference, 1991, 715-720.

3. Pradhan, V.R.; Tierney, J.W.; Wender, I.; Huffman, G.P.; Energy \& Fuels, 1991, 5, 497 507.

4. Lee, P.A.; Citrin, P.H.; Eisenberger, P.; Kincaid, B.M.; Rev. Mod. Phys., 1981, 53, 769- 
806.

5. Greegor, R.B.; Lytle, F.W.; J. of Catalysis, 1980, 63, 476-486.

6. Kundig, W1; Bömmel, H.; Constabaris, G.; Lindquist, R.H.; Phys. Rev., 1966, 142, 327.

7. Huffman, G.P., Ganguly, G., Taghiei, M.; Huggins, F.E. and Shah, N.; ACS Div. of Fuel Chemistry Preprints, 1991, 36(2), 561-569. 


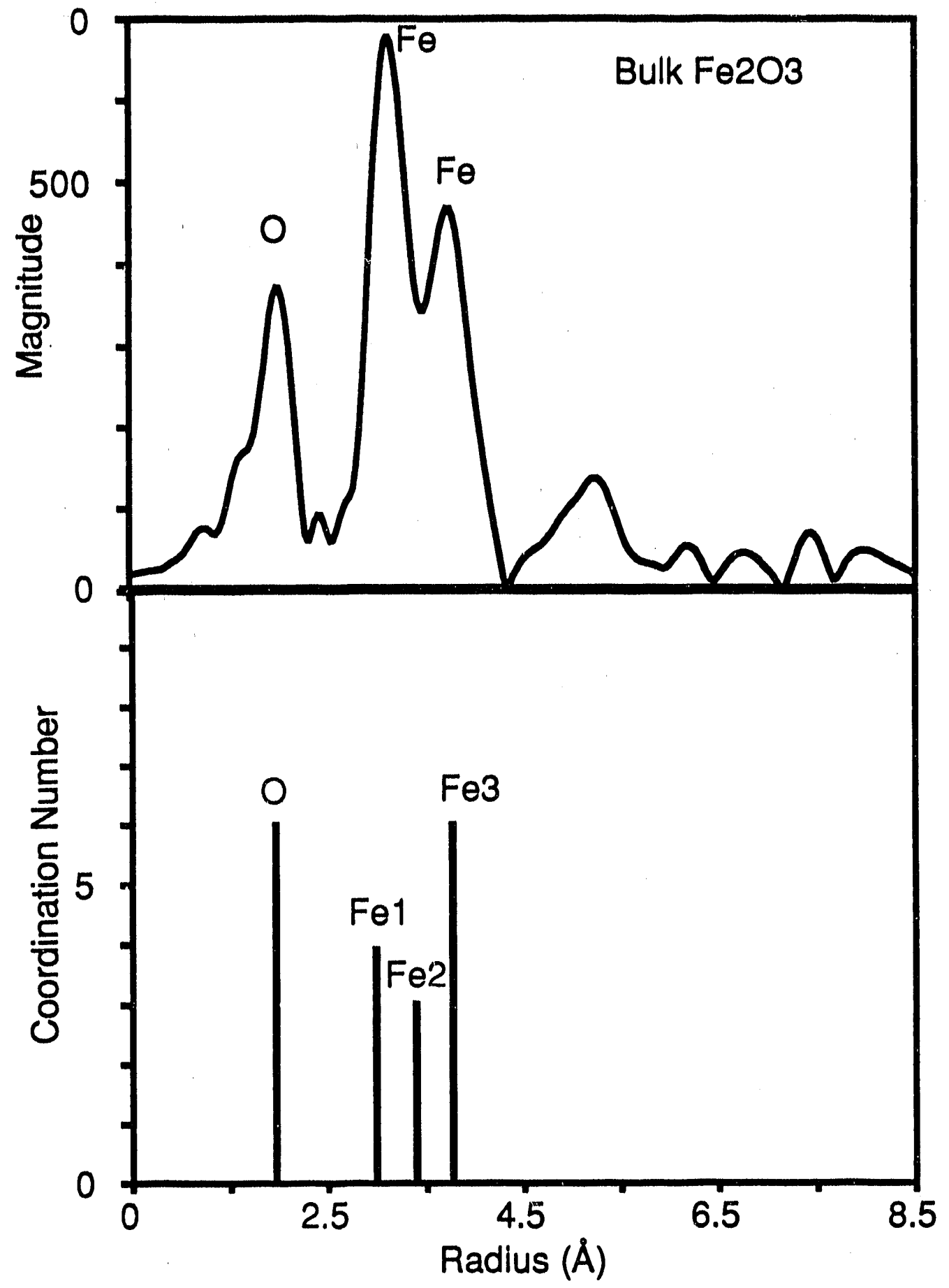

Figure 1. Comparison of the RSF of bulk hematite to the radial distribution function. 


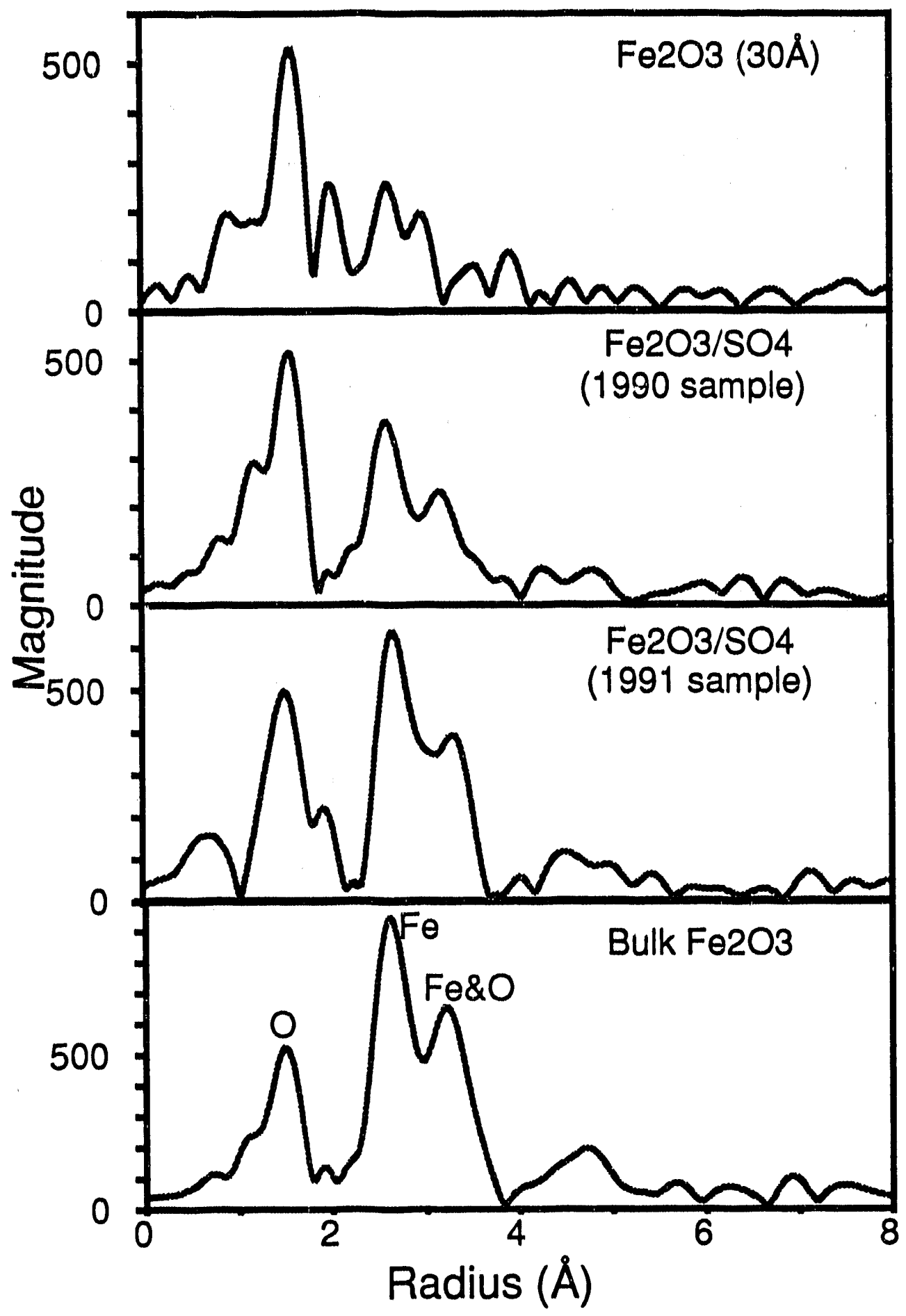

Figure 2. Comparison of the RSF of bulk hematite to those of several $\mathrm{Fe} 2 \mathrm{O} 3$ catalysts. 


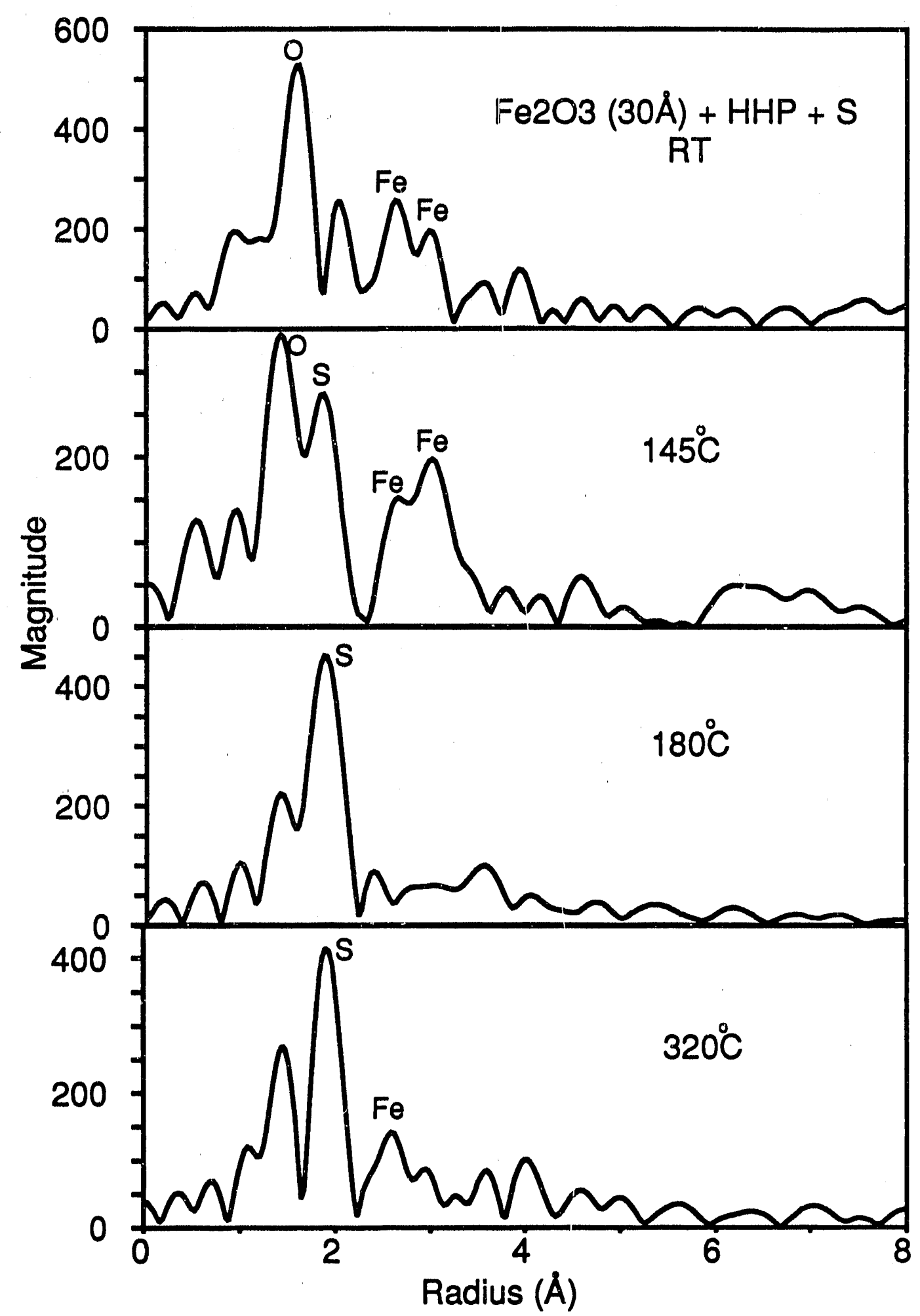

Figure 3. In situ RDF's of $\mathrm{Fe} 2 \mathrm{O} 3$ (30Å) from Mach I Inc. Mixed with hexahydropyrene at indicated temperetures. 


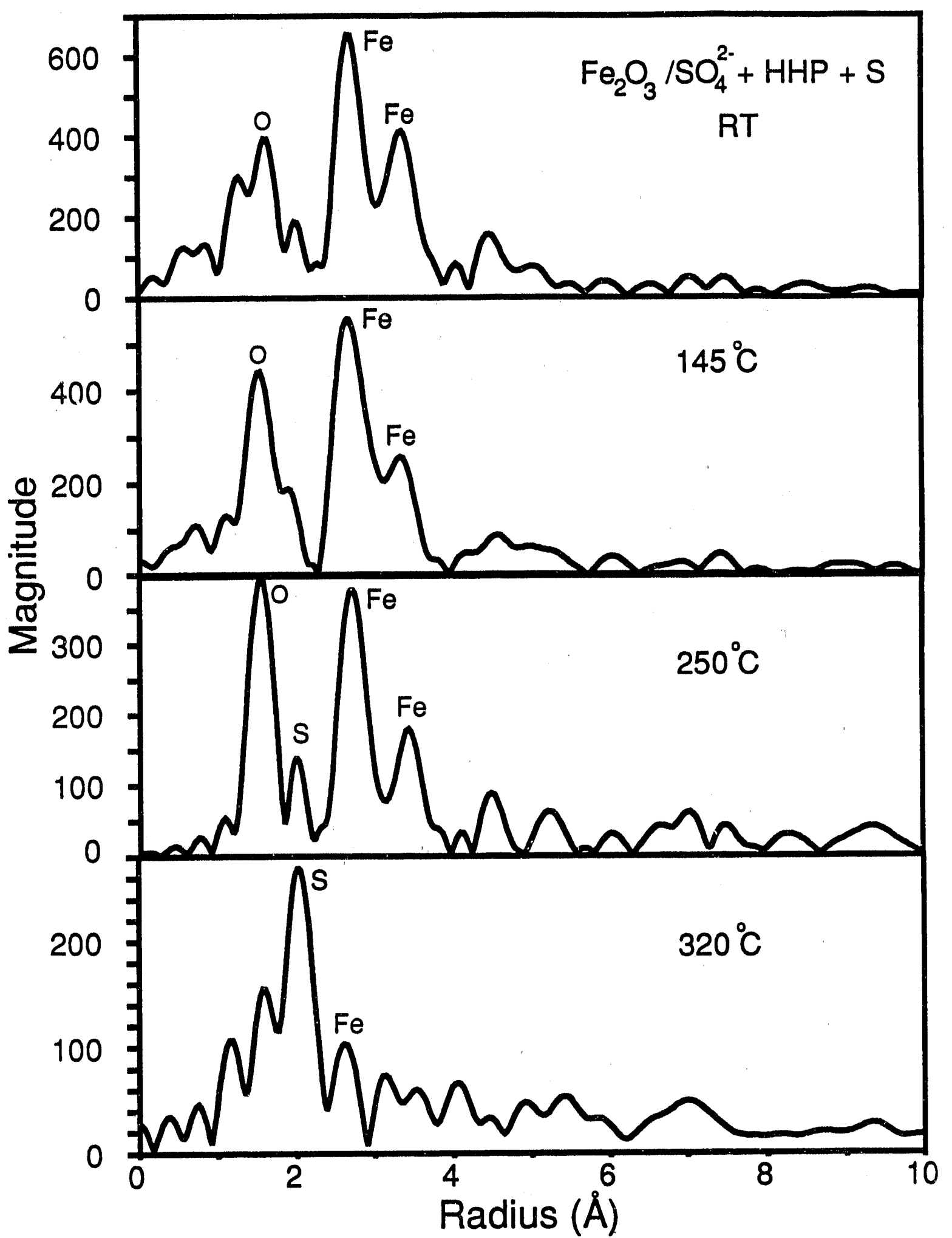

Figure 4. In situ RDF's of Fe2O3/SO4 Catalyst mixed with hexahydropyrene plus elemental sulfur at indicated temperatures.

35 


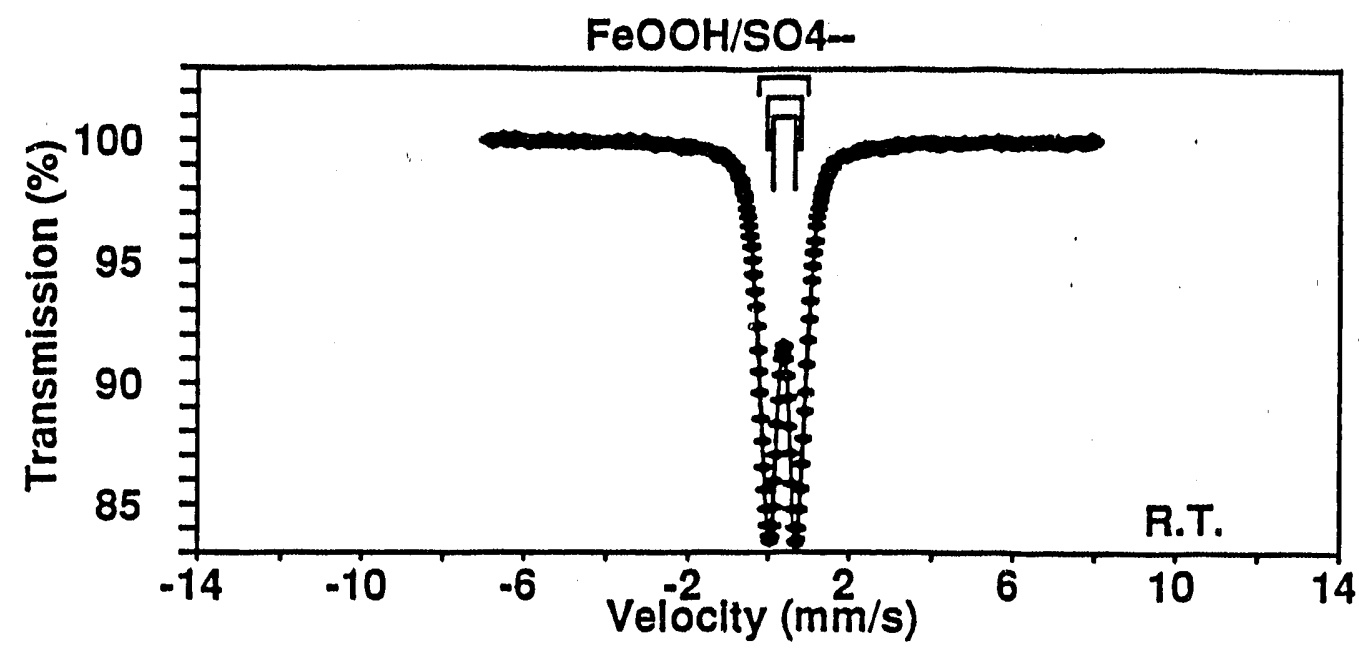

$100 \%<$

Fe2O3/SO4-

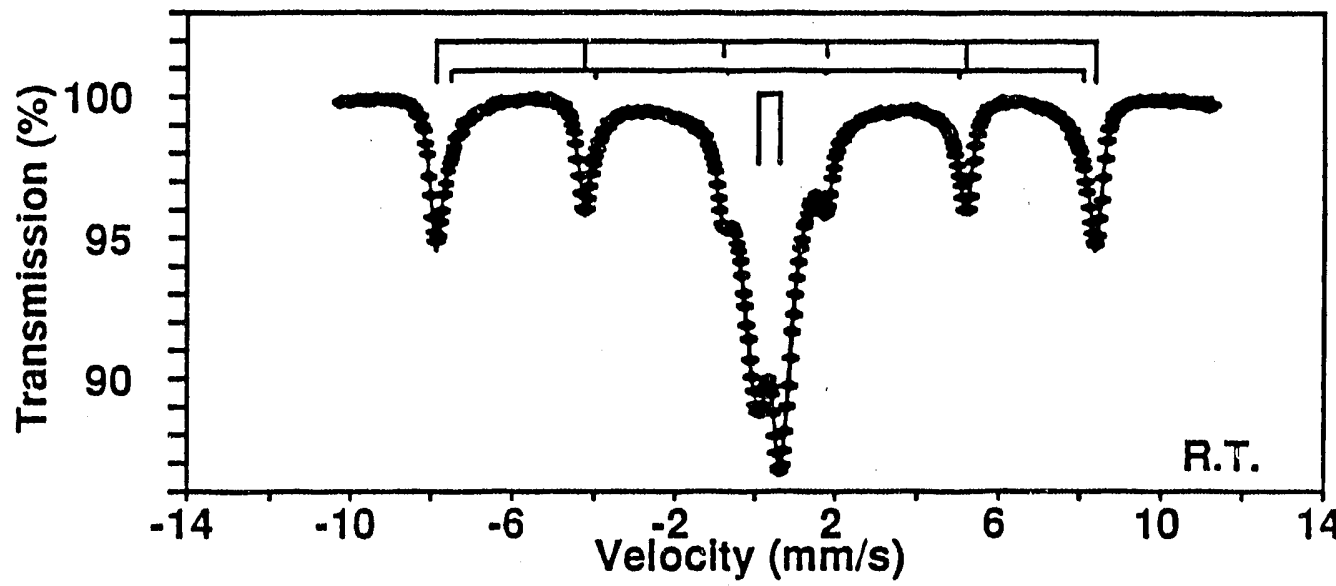

$60 \%<8$

$40 \%>8$

Fe2O3/SO4--

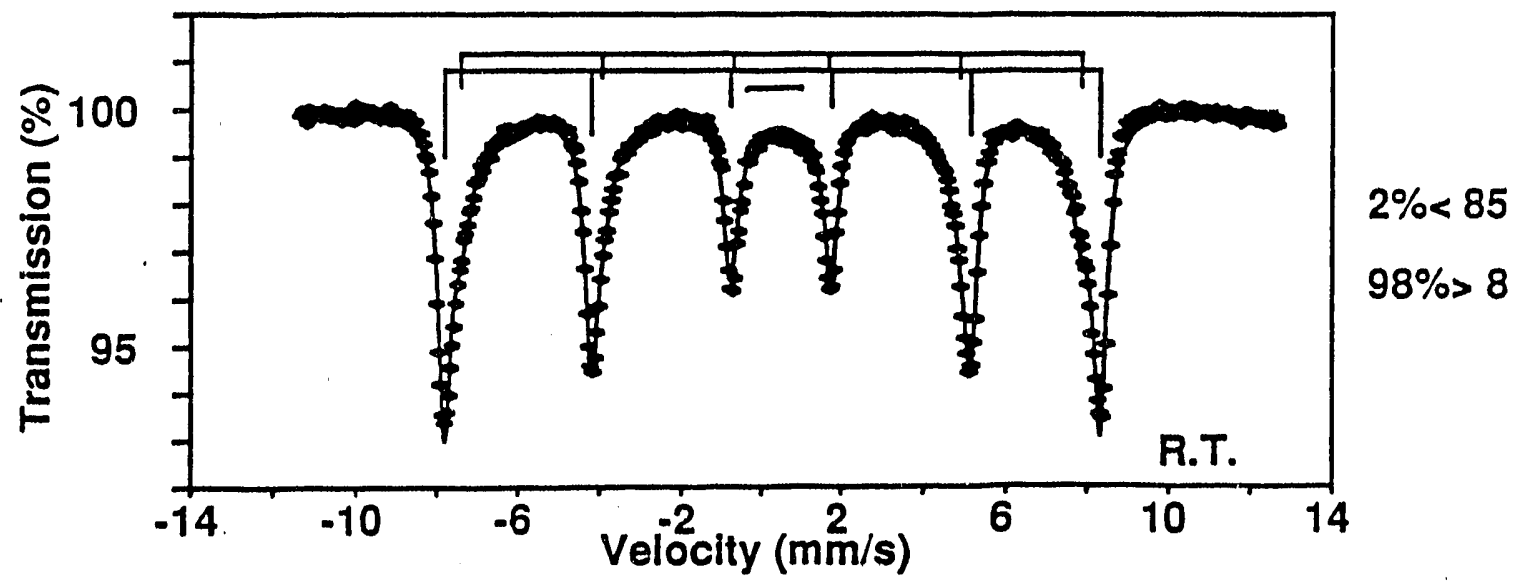

Figure 5. Mössbauer spectra of sulfated ferric oxide catalysts. Before calcination, only a quadrupole doublet is observed, while the two calcined samples exhibit significantly different quadrupole and magnetic percentages. 
Table 1. results of least-squares analysis of the back transforms of the iron shell peaks of several catalysts. Standard deviation are \pm 0.02-0.05 $\AA$ for distances, and \pm 0.5-1.0 for coordination numbers.

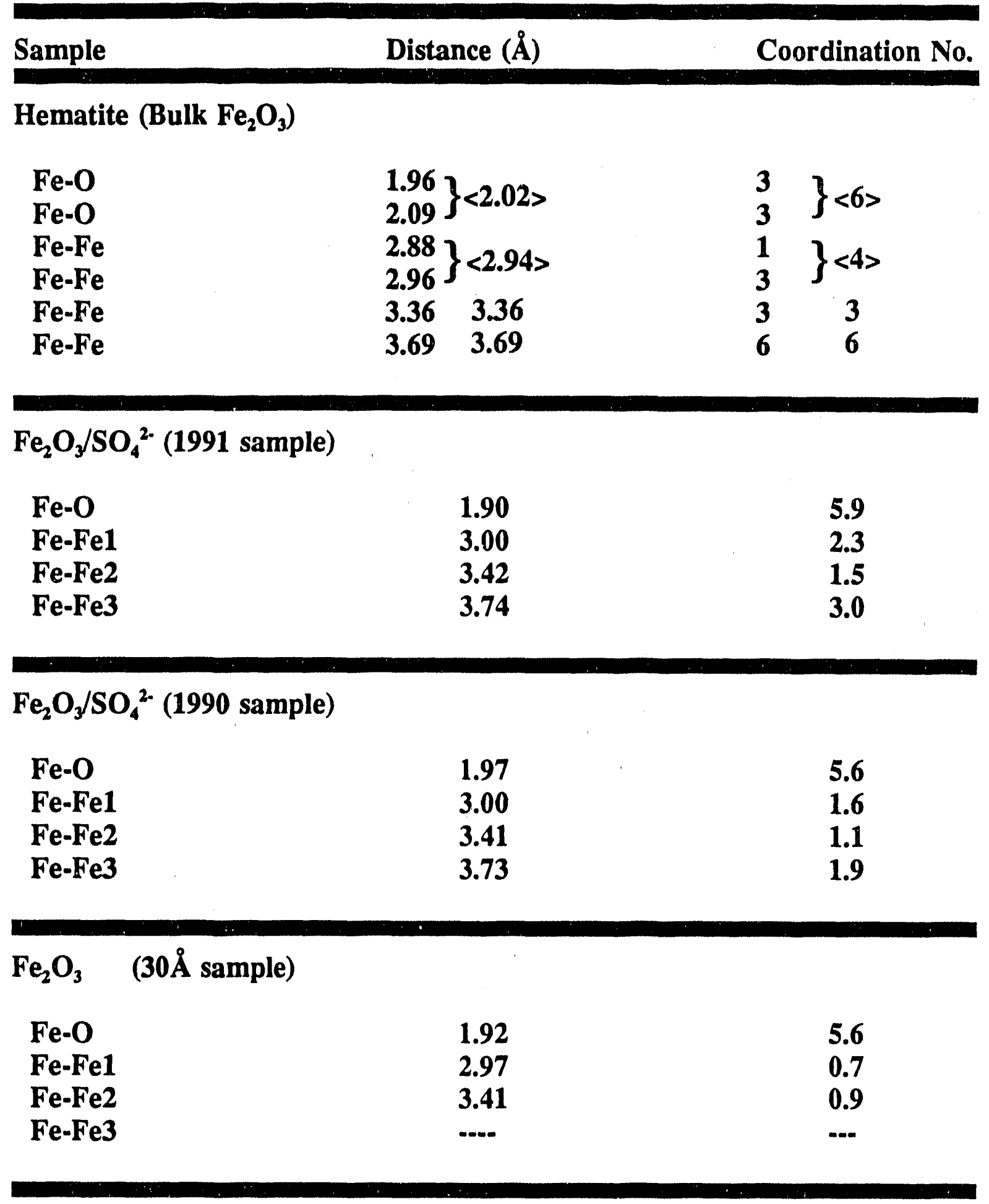


Tab. 2a. Coordination Number and Distance of the Neighbor Shells of Iron Standards.

\begin{tabular}{|c|c|c|c|}
\hline Sample & Shell & Distance & Coord.number \\
\hline $\mathrm{Fe}_{2} \mathrm{O}_{3}$ standard & $\begin{array}{l}F_{\theta}-O \\
F_{\theta}-F_{\theta} \\
F_{\theta}-F_{\theta} \\
F_{\theta}-F_{\theta}\end{array}$ & $\begin{array}{l}1.98 \\
2.95 \\
3.37 \\
3.70\end{array}$ & $\begin{array}{l}6 \\
4 \\
3 \\
6\end{array}$ \\
\hline $\mathrm{Fe}_{1-x} \mathrm{~S}$ & $\begin{array}{l}F_{\theta}-S \\
F_{\theta}-F_{\theta}\end{array}$ & $\begin{array}{l}2.48 \\
3.00\end{array}$ & $\begin{array}{l}6 \\
4\end{array}$ \\
\hline $\mathrm{FeS}_{2}$ & $\begin{array}{l}F_{\theta}-S \\
F_{\theta}-F_{\theta} \\
F_{\theta}-F_{\theta}\end{array}$ & $\begin{array}{l}2.26 \\
3.43 \\
3.82\end{array}$ & $\begin{array}{c}6 \\
6 \\
12\end{array}$ \\
\hline
\end{tabular}


Tab. 2b. Coordination Number and Distance of the Nearest Nelghboring Shells of In-situ $\mathrm{Fe}_{2} \mathrm{O}_{3} / \mathrm{SO}_{4}$ Catalysts with HHP.

\begin{tabular}{|c|c|c|c|}
\hline Sample & Shell & Distance & Coord.Number \\
\hline $\begin{array}{l}30 \text { A sample at: } \\
\text { a.Room temp. }\end{array}$ & $\begin{array}{l}F \theta-O \\
F \theta-S(?) \\
F \theta-F \theta \\
F_{\theta}-F_{\theta}\end{array}$ & $\begin{array}{l}1.98 \\
2.39 \\
2.99 \\
3.41\end{array}$ & $\begin{array}{l}4.6 \\
1.3 \\
0.7 \\
0.9\end{array}$ \\
\hline b. $110 \mathrm{C}$ & $\begin{array}{l}\mathrm{F}_{\theta}-\mathrm{O} \\
\mathrm{F}_{\theta}-\mathrm{S} \\
\mathrm{F}_{\theta}-\mathrm{F}_{\theta} \\
\mathrm{F}_{\theta}-\mathrm{F}_{\theta}\end{array}$ & $\begin{array}{l}1.95 \\
2.38 \\
3.05 \\
3.53\end{array}$ & $\begin{array}{l}4.1 \\
1.3 \\
0.9 \\
0.5\end{array}$ \\
\hline c. $180 \mathrm{C}$ & $\begin{array}{l}\mathrm{Fe}-\mathrm{O} \\
\mathrm{Fe}-\mathrm{S}\end{array}$ & $\begin{array}{l}2.09 \\
2.46\end{array}$ & $\begin{array}{l}3.9 \\
3.7\end{array}$ \\
\hline $\begin{array}{l}\text { d. } 320 \mathrm{C} \text {, then cooled } \\
\text { to room temp. }\end{array}$ & $\begin{array}{l}F_{\theta}-\mathrm{S} \\
\mathrm{F}_{\theta}-\mathrm{Fe}\end{array}$ & $\begin{array}{l}2.46 \\
3.02\end{array}$ & $\begin{array}{l}6.5 \\
5.5\end{array}$ \\
\hline $\begin{array}{l}\text { U.Pitt } \mathrm{Fe}_{2} \mathrm{O}_{3} / \mathrm{SO}_{4} \text { at: } \\
\text { a. Room temp. }\end{array}$ & $\begin{array}{l}F_{\theta}-O \\
F \theta-F \theta \\
F \theta-F \theta \\
F \theta-F \theta\end{array}$ & $\begin{array}{l}1.96 \\
3.00 \\
3.42 \\
3.74\end{array}$ & $\begin{array}{l}6.6 \\
2.3 \\
1.5 \\
3.0\end{array}$ \\
\hline b. $250 \mathrm{C}$ & $\begin{array}{l}\mathrm{F}_{\theta-O} \\
\mathrm{~F} \theta-\mathrm{S} \\
\mathrm{F}_{\theta}-\mathrm{F}_{\theta} \\
\mathrm{F} \theta-\mathrm{F}_{\theta} \\
\mathrm{F} \theta-\mathrm{F}_{\theta}\end{array}$ & $\begin{array}{l}1.92 \\
2.41 \\
3.00 \\
3.40 \\
3.78\end{array}$ & $\begin{array}{l}4.2 \\
0.9 \\
1.7 \\
0.4 \\
0.6\end{array}$ \\
\hline $0.320 \mathrm{C}$ & $\begin{array}{l}F_{\theta}-S \\
F_{\theta}-F_{\theta}\end{array}$ & 2.46 & 5.4 \\
\hline $\begin{array}{l}\text { d. Room temp. } \\
\text { after } 320 \mathrm{C}\end{array}$ & $\begin{array}{l}F_{\theta}-S \\
F_{\theta}-F_{\theta}\end{array}$ & $\begin{array}{l}2.47 \\
2.99\end{array}$ & $\begin{array}{l}7.0 \\
6.0\end{array}$ \\
\hline
\end{tabular}

"* signal was weak. 


\title{
Project I.4 Catalyst Dispersion and a Model Compound
}

\author{
E.M. Eyring, D.A. Sommerfeld and Y.-H. Huang \\ University of Utah
}

\begin{abstract}
At the CFFLS technical meeting in Lexington, Kentucky (August 12-14, 1991) we reported an electron probe microanalysis (EPMA) study of the dispersion of a bimetallic iron/molybdenum catalyst in demineralized Blind Canyon coal before and after hydrotreatment. Our EPMA images indicated that before hydrotreatment the impregnation of the coal particles with iron and molybdenum advanced no further than the margins of the coal particles regardless of whether impregnation was by the incipient wetness technique or by ultrasonication. After hydrotreatment the EPMA images showed that iron and molybdenum were dispersed fairly uniformly throughout the interior of the coal particles. Members of the audience raised two objections to this study:
\end{abstract}

1) More coals should be similarly tested to confirm (or disprove) the generality of the above conclusions; and

2) Demineralization probably alters the coal structure and also requires impractically expensive hydrofluoric acid.

During the present Quarter these experiments have been repeated with three Argonne coals: Pittsburgh \#8, Wyodak and Blind Canyon coal. These coals were used as received, i.e. they were not demineralized, and they were impregnated with iron and molybdenum by incipient wetness and by ultrasonication. The EPMA image obtained before and after hydrotreatment of the undemineralized Blind Canyon coal were consistent with those obtained previously with demineralized Blind Canyon coal; iron and molybdenum were localized around the border of the coal particles before hydrotreatment and entered the coal particles during hydrotreatment. Results obtained with Pittsburgh \#8 coal closely resemble results obtained with Blind Canyon coal as would be expected since both are high volatility bituminous coals. Wyodak coal shows penetration of iron and molybdenum into the coal particles before hydrotreatment. The higher polarity and porosity of this sub-bituminous coal (than of Blind Canyon and Pittsburgh \#8 coals) may account for coal rank playing a role in catalyst dispersion.

A manuscript entitled "Spectroscopic Studies of Coal Maceral Depolymerization Effected by an Iron-Based Catalyst" was revised to meet the objections of referees and resubmitted to FUEL in late August. It has since been accepted for publication.

Dr. Yo-Hsin Huang, an organometallic chemist, has synthesized 2.2 grams of a model coal compound described by M. Farcasiu and C. Smith, Energy \& Fuels 1991, 5, 83. Dr. Huang used a synthetic procedure supplied by workers at $\$$ andia Laboratories in Albuquerque, 
NM. Minor refinements that Dr. Huang added to the procedure are noted in the following outline:

All reactions were conducted under a dry nitrogen atmosphere using Schlenk techniques. Reaction of bibenzyl with 1- naphthyl chloride in carbon disulphide in the presence of aluminum chloride gave naphthyl bibenzyl ketone. Treatment of naphthyl bibenzyl ketone with hydrazine hydrate and potassium hydroxide in diethylene glycol at $230^{\circ} \mathrm{C}$ for $5 \mathrm{~h}$ generated the model compound 4-(-1-naphthyl methyl) bibenzyl. Sandia scientists reported that the diethylene glycol (b.p. $245^{\circ} \mathrm{C}$ ) was distilled off at reduced pressure. Alternatively, the diethylene glycol can be easily removed as follows: After the reaction mixture was allowed to cool to room temperature, the contents of the flask were poured into a separatory funnel containing $50 \mathrm{~mL}$ of water. The diethylene glycol/water mixture was extracted $(3 \times 70 \mathrm{~mL})$ with benzene and all benzene fractions were combined. Then, the benzene solution was washed with distilled water $(2 \times 30 \mathrm{~mL})$, dried $\left(\mathrm{MgSO}_{4}\right)$, and filtered. Removal of the solvent by rotary evaporation gave a viscous yellow-brown oil which was subsequently purified by column chromatography on silica gel. 


\title{
Project I.5 Characterization of Catalysts Used in Coal Hydrogenation Systems
}

\author{
Nelly M. Rodriguez and R. Terry K. Baker \\ Auburn University
}

During this period we have undertaken a closer examination of various catalysts supplied to us from the Universities of Pittsburgh and West Virginia. These studies have been conducted in the controlled atmosphere electron microscope, where samples of the iron containing sulfur catalysts were dispersed on transmission specimens of single crystal graphite as a support media. This combination was subsequently reacted in the environmental cell in the presence of hydrogen and changes in appearance in both the catalyst and the graphite support followed continuously.

\section{i. Fe2O3.SO4 with 0.5\% Mo (From University of Pittsburgh)}

When these specimens were reacted in 0.3 Torr hydrogen, the initial needle-like structures were observed to undergo disintegration after holding the temperature at $330^{\circ} \mathrm{C}$ for 30 minutes. This reconstruction phenomena led to the formation of very small particles, 1$2 \mathrm{~nm}$ in diameter. On continued reaction at this temperature, it became apparent that small pits were starting to form in the graphite basal plane around each of the catalyst particles and in some cases this action was seem to occur at some distance from a given catalyst particle. After about a further 30 minutes period inspection of the pits showed that they have expanded both laterally and become progressively deeper. It was also clear that the sides of the pits had taken on definite orientations to create a hexagonal outline as presented in Figure 1. If the reaction temperature was maintained at about $350^{\circ} \mathrm{C}$, then over a prolonged period of time extensive attack of the graphite was observed. It was significant, however, that when the temperature was progressively raised to $600^{\circ} \mathrm{C}$, there was an appreciable decrease in the rate of attack. In order to eliminate any adverse effects of the electron beam on the reaction, in most cases the beam was turned off while the specimen was reacted and only examined on 
an intermittent basis.

\section{1i. Fe $\mathrm{S}_{\mathrm{y}}$ \#1 Catalyst (West Virginia University)}

This material exhibited a sponge-like texture in its initial state and underwent a structural transformation at about $210^{\circ} \mathrm{C}$ to form an array of very small particles, $2.5 \mathrm{~nm}$ in diameter and an assortment of tape-like structures. On raising the temperature to $300^{\circ} \mathrm{C}$ an amazing transformation in appeurance of the specimen was observed as pits started to form all over the basal plane. It was interesting to find that the shape of the pits was quite different to that observed with the previous catalyst system, in this case, the outline being rectangular in form. Furthermore, close inspection of the edges of the pit showed that they were coated with a thin layer of the catalyst material, i.e. the catalyst was wetting and spreading along edge regions and appeared to dictate the direction of attack. These events are shown schematically in Figure 2. If the system was maintained at this temperature, then the reaction continued to become extremely wide spread with the result that portions of the graphite substrate underwent disintegration. As with the previous system, however, if the temperature was raised to $600^{\circ} \mathrm{C}$ the catalyst tended to loose its activity.

\section{iii. $F e_{x} S_{y} \# 2$ Catalyst (West Virginia University)}

In many regards, the appearance and subsequent behavior of this material was similar to that described for the catalyst, Fe203.SO4 (University of Pittsburgh). At room temperature, it exhibited a needle-like morphology, which underwent a transformation at $150^{\circ} \mathrm{C}$ to produce very small particles. On heating the system to $350^{\circ} \mathrm{C}$, pits were produced all over the basal plane, tending to acquire a hexagonal outline (Figure 1). As with the other catalyst systems, loss of activity was observed when the temperature was raised to around $600^{\circ} \mathrm{C}$. 


\section{DISCUSSION}

It is clear that the controlled addition of sulfur atoms to iron can have a profound effect on the manner by which the metal catalyzes the hydrogenation of graphite. In its pure form, iron does not react with graphite in the presence of hydrogen until the temperature is increased to $890^{\circ} \mathrm{C}$ and under these conditions particles located at edge regions are observed to remove carbon atoms via a channeling action which is the result of particles moving across the basal plane. In the current experiments hydro-gasification of graphite is found to take place by a pitting action at temperatures as low as $300^{\circ} \mathrm{C}$, indicating that a different mechanism is operative. It is our belief that certain iron-sulfur compounds are capable of interacting with molecular hydrogen to generate an intermediate which can readily undergo reaction with graphite at these temperatures. In this context, it is worth considering the phenomena of hydrogen spillover which is known to occur with certain supported metal catalysts and may be an important step in catalyzed coal hydrogenation, although economic considerations naturally preclude the use of the catalysts likely to be most effective. Up to the present time the importance of spillover in this system has not been widely appreciated.

In future studies we intend to characterize the chemical state of the catalysts described above by the use of in-situ electron diffraction techniques. Using this approach it should be possible to follow changes in the chemical state of the respective systems and hopefully correlate these with changes in the catalytic reactivity. 


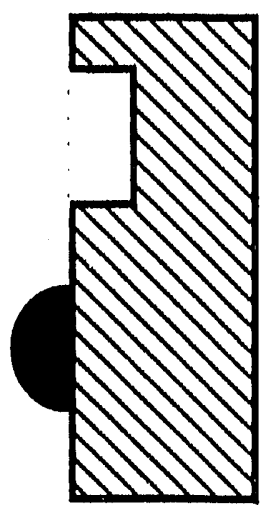

3
$\frac{3}{2}$
0
0
0
0
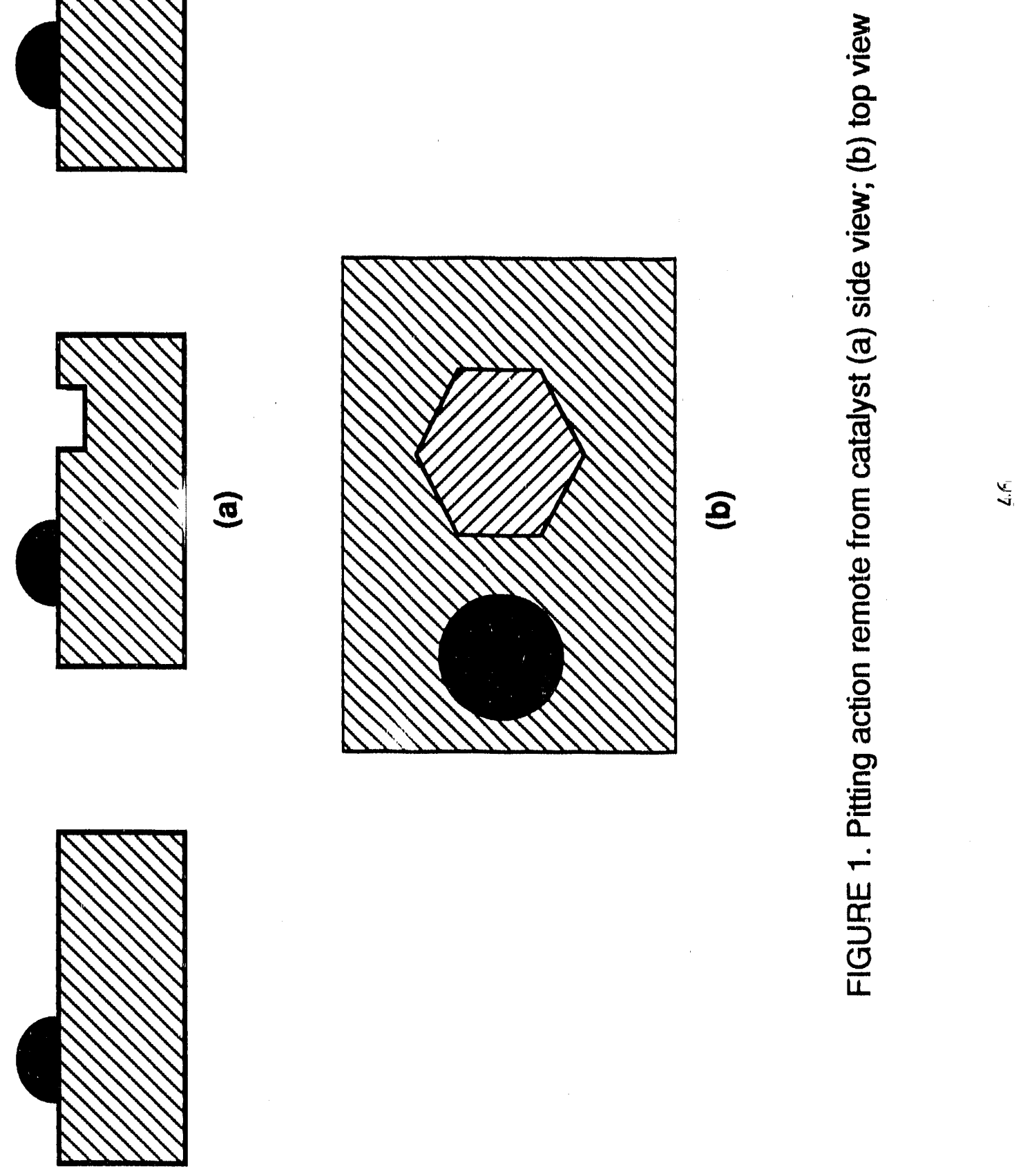

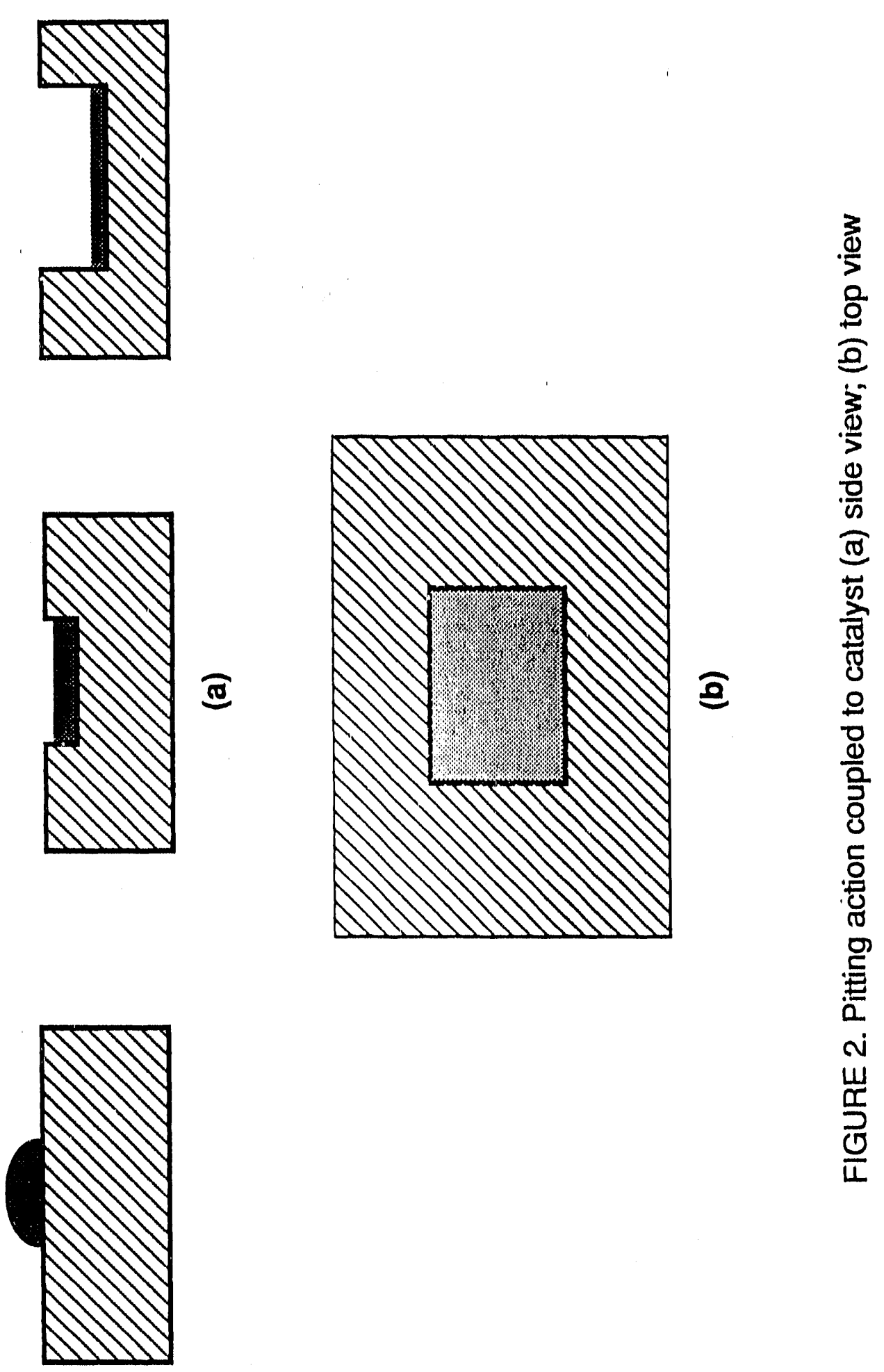


\title{
Project I.6 Test Reactor for Catalytic Coal Liquefaction
}

\author{
John Zondlo, Chemical Engineering \\ Al Stiller, Chemical Engineering \\ Peter Stansberry, Chemical Engineering \\ Dady Dadyburjor, Chemical Engineering \\ West Virginia University
}

\section{TEST REACTOR}

Construction of a batch coal liquefaction reactor is completed. Several liquefaction reactions have been run to test the temperature controller and agitation mechanism for mechanical problems. The reactor system has operated without failure. The design of the gas-flow-through test reactor will now incorporate the agitation mechanism of the batch system. The design of the tubing bomb and the high pressure gas delivery and condenser system for the flow-through reactor system is now under way.

The experimental plan for the batch reactor system has been developed. Particular emphasis has been placed on the procedures whereby the liquefaction products are separated and categorized. A method employed by Robert Keogh and co-workers at the University Kentucky was followed initially using a Western Kentucky coal sample provided by Mr. Keogh. A summary of this work-up procedures is shown in Figure 1 and described as follows:

IJ's Experimental Liquefaction of Coal

Coal is liquefied in the CAER $50 \mathrm{ml}$ batch reactors using a reaction temperature of $385^{\circ} \mathrm{C}$, a 15 minute residence time, tetralin as a solvent and a hydrogen atmosphere ( $800 \mathrm{psig}$, ambient). The reactor was charged with 5 grams of dried coal and 7.5 grams of tetralin (Keogh, 1991).

UK's Solubility Class Separations of Reaction Products

The reactor products are separated and washed into a dry Soxhlet thimble with 
berzene which is set up in a Soxhlet extraction apparatus. The products are extracted for 24-48 hours until the benzene is clear. The benzene is removed from the soluble fraction via rota-evaporation under reduced pressure. To the benzene solubles, which are usually viscous liquids, no more than $1 \mathrm{ml}$ of tetrahydrofuran (THF) is added to reduce the viscosity prior to the precipitation step. The asphaltenes are separated from the benzene solubles via precipitation with normal pentane. A minimum of $500 \mathrm{ml}$ of normal pentane is added to the benzene solubles while stirring vigorously. The resulting precipitate is a "fluffy" brown solid. The oil (normal pentane solubles), which contains tetralin and its reaction products (naphthalene, methyl indane, decalin, etc.) are determined by difference between the total benzene soluble yields (gas, oils and asphaltenes) and the asphaltene plus gas yield.

The dried and weighed thimble is then extracted with pyridine until the pyridine is clear in a Soxhlet apparatus. This step yields the preasphaltene and insoluble organic material (IOM) content of the reaction products.

Several attempts were made to utilize this procedure for the separation of coal liquefaction products of the Kentucky coal samples reacted under identical conditions as above. Results from these tests are shown in Table 1. It is observed that total conversion and the asphaltene and preasphaltene fractions differed radically from Keogh's data. Problems were encountered in trying to wash out all the reaction products from the reactor using benzene. Thus the total mass balance closure was erratic. Furthermore, the pyridine extraction left a coating of insoluble coal liquids on the soxhlet that could not be recovered. This problem led to a higher total conversion than Keogh obtained. Finally, due to its toxicity, benzene is no longer a preferred solvent of choice. Hence an alternate work-up scheme previously published has been adopted by us for all subsequent product evaluation. The procedure is as follows:

WVU's Solubility Class Separations of Reaction Products

The procedure for product work-up is shown in Figure 2. Here the emphasis is placed on obtaining data on total conversion and oil production since these data are 
the most relevant for catalyst screening tests. Following reaction, the reactor products are separated and washed into a dry Soxhlet thimble with THF which is set up in a Soxhlet extraction apparatus. The products are extracted for up to 72 hours until the THF is clear. The dried and weighed material in the thimble is the IOM fraction. The THF is recovered from the soluble fraction via rota-evaporation under reduced pressure in a water bath. Following evaporation of the THF, hexane is added to the coal liquid/tetralin mixture and refluxed for two hours. The insoluble material from this step is removed via filtration and represents the preasphaltene/asphaltene fraction. The liquid filtrate containing the tetralin and oils is again subjected to rota-evaporation whereupon the tetralin and hexane are removed leaving the oil fraction to be determined.

This procedure was applied to the same Western Kentucky coal used above. Results from these tests indicate that the procedure is far more easily performed and the data are much more reproducible. This procedure was used for product evaluation of the liquefaction results reported below.

\section{Liquefaction Results:}

\section{a) Stirred Autoclave Reactor}

Liquefaction reactions were performed in a $450 \mathrm{ml}$ stirred autoclave reactor. For these tests, the DECS-17 coal was used. All experiments were done using 10 grams of coal and $80 \mathrm{ml}$ of tetralin. The data reported below represent the baseline case where no catalyst has been added. As shown in Table 2, two temperatures $\left(250^{\circ} \mathrm{C}\right.$ and $\left.350^{\circ} \mathrm{C}\right)$, two gas phase pressures (300 psig and 1000 psig cold), and two different gas atmospheres $\left(\mathrm{H}_{2}\right.$ and $\left.\mathrm{N}_{2}\right)$ were tested. All reactions were run for 1 hour. The data reveal that for this set of process conditions, temperature is the only significant process variable. Total yield averaged around $19 \%$ at $250^{\circ} \mathrm{C}$ and around $59 \%$ at $350^{\circ} \mathrm{C}$. Thus these non-catalytic reactions are essentially thermal in nature. Currently, iron based catalysts are being prepared for testing.

\section{b) Tubing Bomb Reactor}

The same DECS-17 coal was tested in the newly-constructed shaker tubing bomb 
apparatus. Here 3 grams of coal and $5 \mathrm{ml}$ of tetralin were loaded into a $25 \mathrm{ml}$ batch tubing bomb reactor. Two temperatures were used $\left(250\right.$ and $350^{\circ} \mathrm{C}$ ) under 1000 psig (crid) $\mathrm{H}_{2}$ pressure for reaction times of 15 and 60 minutes. Two different agitation speeds were used (300 and $500 \mathrm{rpm}$ ). Results of these non-catalytic tests are shown in Tables 3 and 4. As shown in the tables, the data reveal that temperature and time are the two significant process variables, while agitation rate plays a lesser role in conversions. It may be noted that agitation rate may be more significant in the oil yields. Curiously, higher oil yields are found for lower agitation speeds. However, more data points need to be filled in before we can speculate. Also note that total conversion for the 60 minute runs is identical to that obtained in the autoclave reactor.

Work continues with filling the out the baseline data and catalytic runs will be underway shortly. 


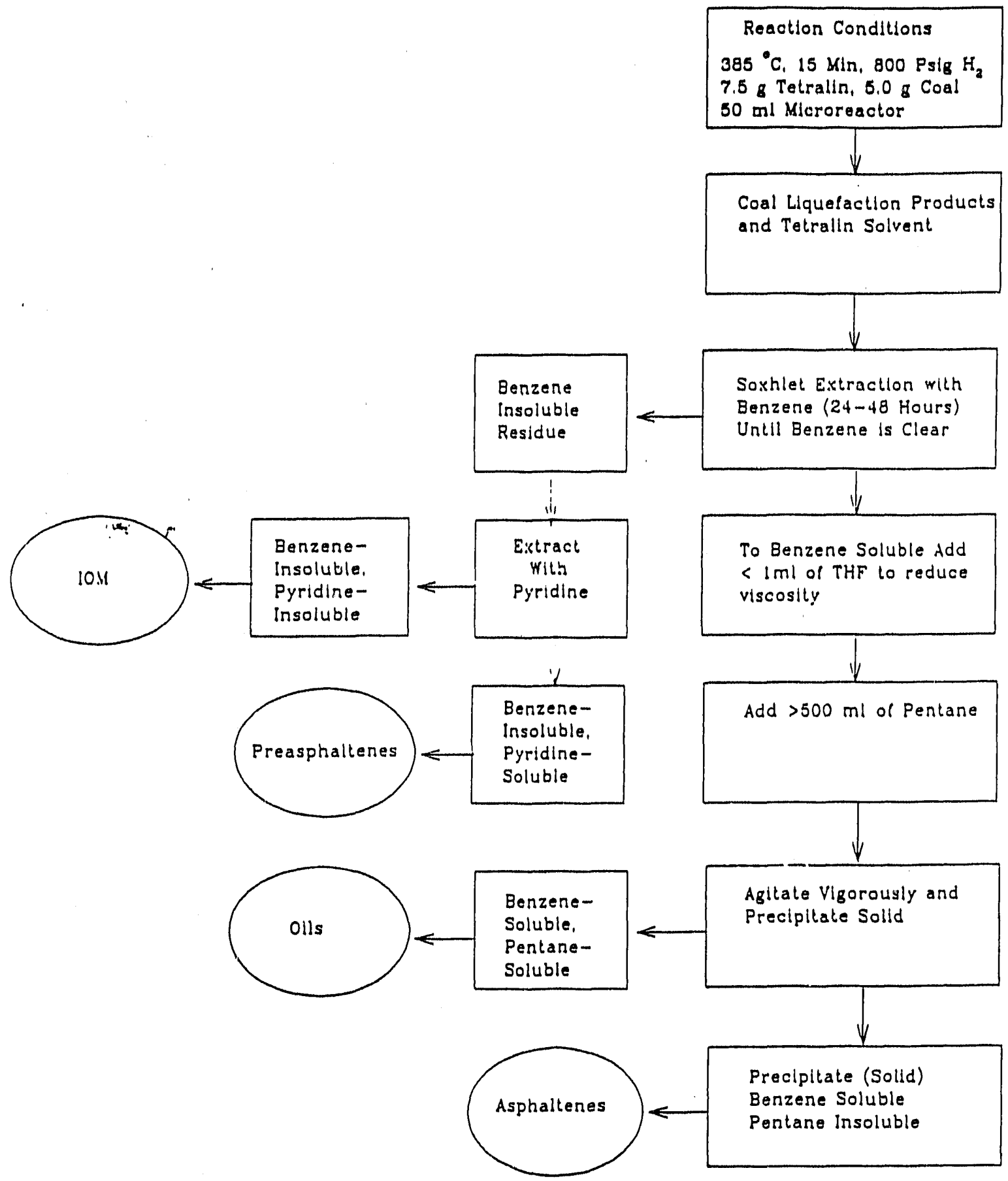

Figure 1. Block diagram of the UK solubility separation scheme. 
FIGURE 2. EXPERIMENTAL SCHEME OF

CATALYTIC COAL LIQUEFACTION AND PRODUCT FRACTIONATION

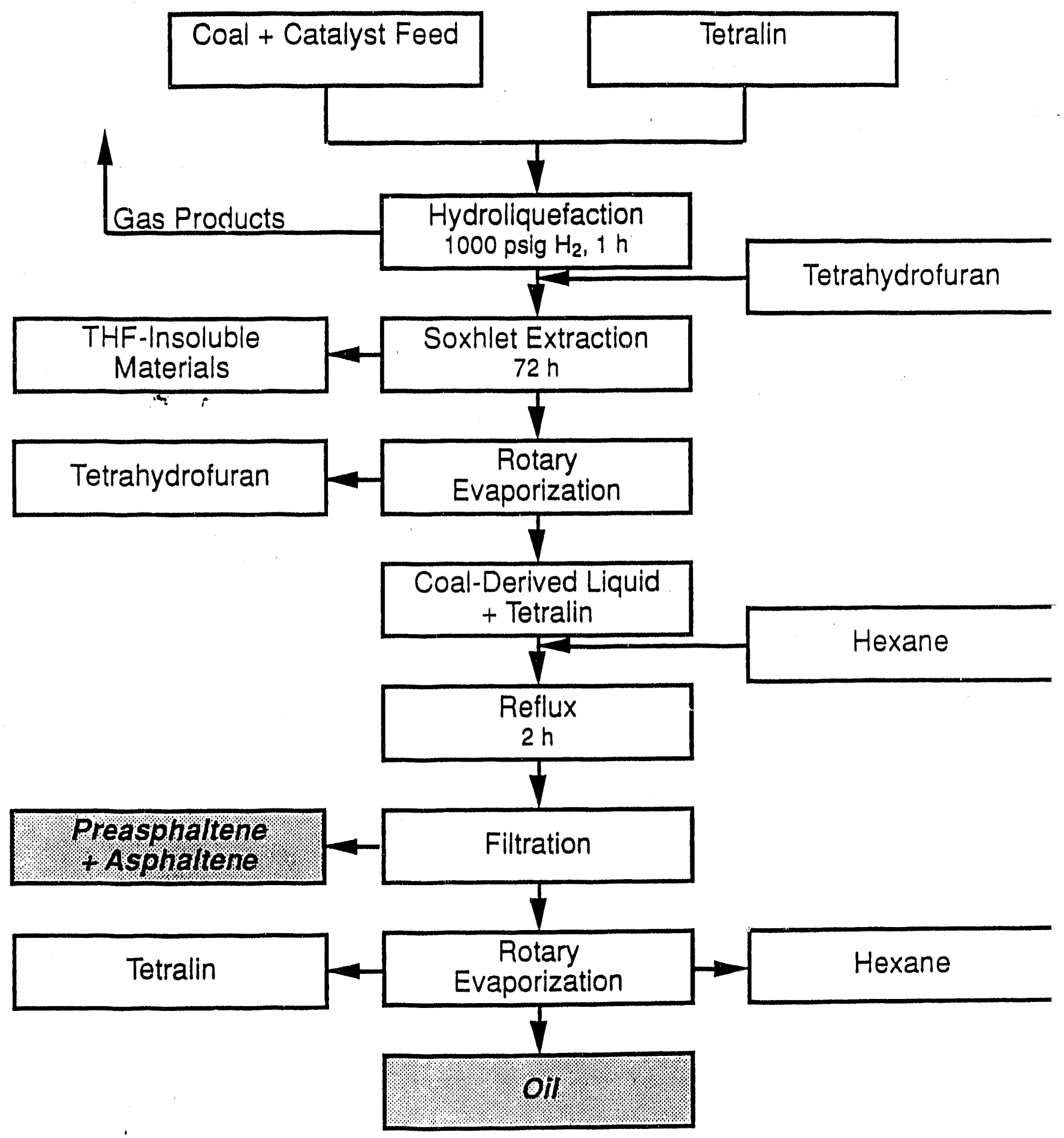




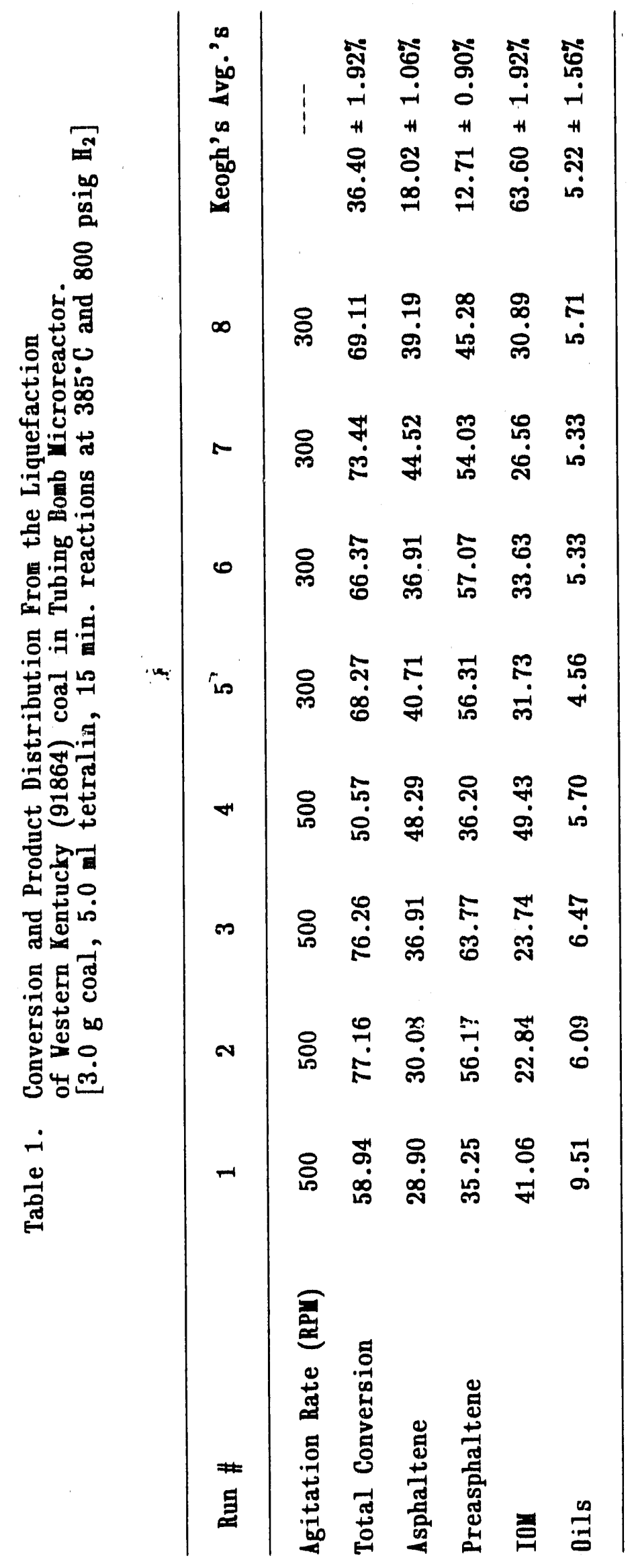




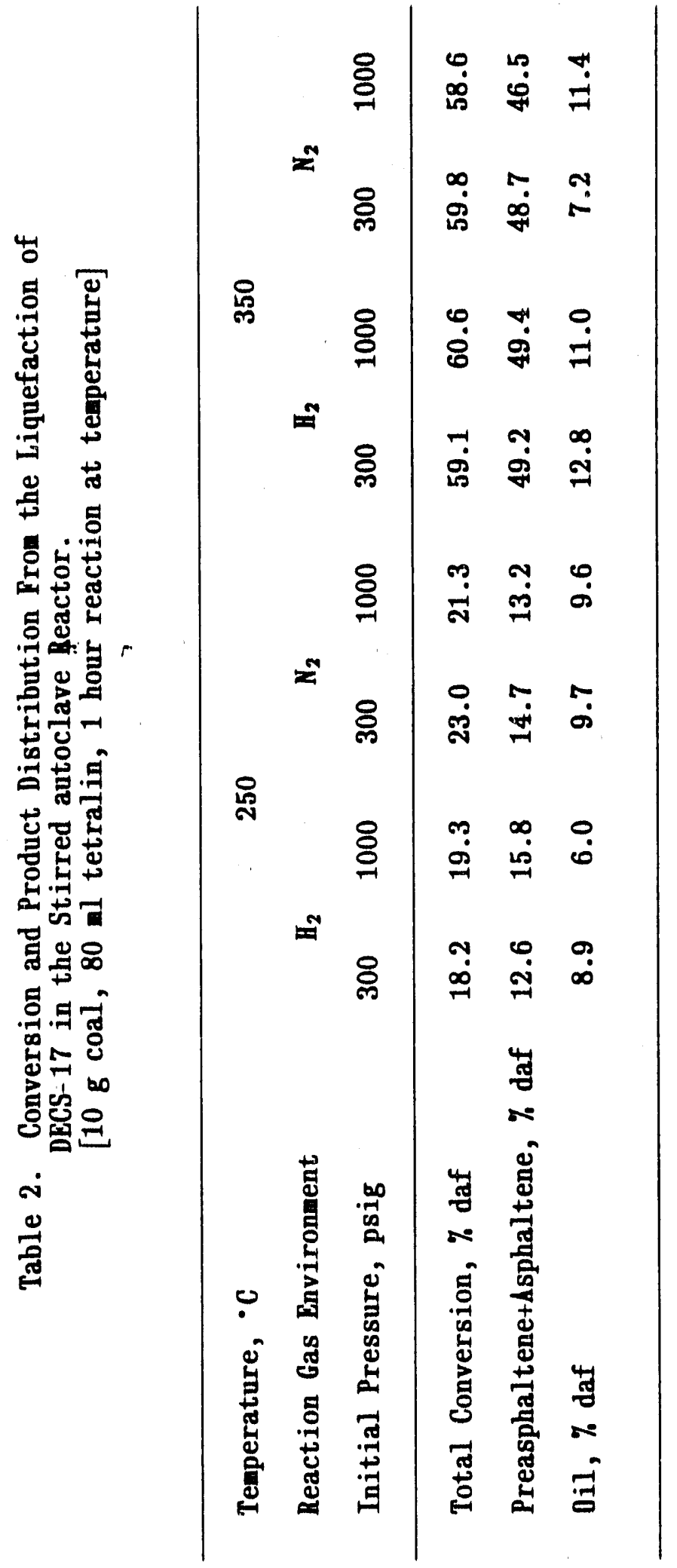


Table 3. Results for Liquefaction of DECS- 17 Coal in Tetralin at $350^{\circ} \mathrm{C}$ and $1000 \mathrm{psig} \mathrm{B}_{2}$ for Various Reaction Times and Agitation Rates

\begin{tabular}{|c|c|c|c|c|}
\hline \multirow{2}{*}{$\begin{array}{l}\text { Agitation Rates } \longrightarrow \\
\text { Time } \\
\downarrow\end{array}$} & \multicolumn{2}{|c|}{$500 \mathrm{RPY}$} & \multicolumn{2}{|c|}{$300 \mathrm{RPH}$} \\
\hline & Conversion & 0ils & Conversion & 0 ils \\
\hline \multirow{2}{*}{60 Minutes } & 57.03 & 6.67 & 60.84 & MYD \\
\hline & 59.22 & 5.56 & NYD & NYD \\
\hline \multirow{2}{*}{15 Minutes } & 41.61 & 5.93 & 41.91 & 8.88 \\
\hline & 42.32 & 5.93 & 39.29 & 10.36 \\
\hline
\end{tabular}

NYD: Not Yet Determined

Table 4. Results for Liquefaction of DECS-17 Coal in Tetralin at $250^{\circ} \mathrm{C}$ and $1000 \mathrm{psig} \mathrm{B}_{2}$ for Various Reaction Times and Agitation Rates

\begin{tabular}{lrrrr}
\hline $\begin{array}{c}\text { Agitation hates } \\
\text { Time }\end{array}$ & Conversion & Oils & Conversion & 0ils \\
& 20.56 & 7.04 & 20.27 & 5.93 \\
60 Minutes & 21.73 & NYD & 20.17 & 7.78 \\
\hline & 13.90 & 11.85 & 17.25 & 8.51 \\
15 Minutes & 13.78 & 10.74 & 16.67 & 7.78 \\
\hline
\end{tabular}

NYD: Not Yet Determined 


\title{
Project I.7 Ferric Sulfide as a Precursor for Coal Liquefaction and Surface Studies of Activity and Selectivity
}

\author{
Dady Dadyburjor, Chemical Engineering \\ Al Stiller, Chemical Engineering \\ John Zondlo, Chemical Engineering \\ Peter Stansberry, Chemical Engineering \\ Charter Stinespring, Chemical Engineering \\ West Virginia University
}

\section{SURFACE STUDIES OF IRON SULFIDE CATALYSTS}

Work during this project period has focused on laboratory setup and a review of the electronic structure of the iron sulfide system. Two ultrahigh vacuum surface analysis systems (non-CFFLS funded) were received and appropriate power lines were installed in the lab. Aralysis capabilities provided by these systems include Auger electron spectroscopy (AES), electron energy loss spectroscopy (EELS), and low energy electron diffraction (LEED). Both AES and EELS provide information on atomic and molecular composition of the surface and near surface layers, while LEED provides structural information. These systems will also include differentially pumped quadrupole mass spectrometers; however, these will be installed on other contract work. A schematic diagram representative of both systems is shown in Figure 1. Future plans call for the addition of X-ray photoelectron spectroscopy (XPS), scanning tunneling microscopy (STM), and interfaced reaction chambers which will allow the study of even more sophisticated chemical reaction processes.

A number of system modifications and small construction projects specifically for the CFFLS program are also in progress. An automated data acquisition system (ADA) has been installed. This modification will significantly improve the quality and reliability of the data while increasing the number of experiments which can be performed. The components for two molecular beam gas sources have been ordered and are being delivered. These sources will allow controlled exposure of the catalysts to reactive gas species such as hydrogen, hydrogen sulfide, and water as well as to model hydrocarbon species. Finally a heated/cooled 
sample manipulator has been ordered. This will allow the sample temperature to be maintained in the range of $123 \mathrm{~K}$ to $1300 \mathrm{~K}$ during reaction and analysis.

Using the ADA, both surface analysis systems are currently being checked out. In this process, a number of AES spectra of pyrite and trollite have been obtained. These spectra will be of considerable value in the contract work since both compounds will serve as standards for the ultrafine iron sulfide catalyst (a pyrite-pyrrhotite mixture), and pyrite will serve as one of several model catalyst surfaces.'

Figure 2 is representative of the AES data obtained to date. Figure 2a shows AES derivative spectra $(\mathrm{dN}(\mathrm{E}) / \mathrm{dE})$ for the $0-160 \mathrm{eV}$ region for troilite and pyrite. It is evident from these spectra that the Fe MVV peak for pyrite is a doublet, while the doublet nature of this peak for troilite is much less pronounced. Conversely, the doublet nature of the S LVV peak is more evident for troilite than for pyrite. These differences may be seen in somewhat greater detail in Figures $2 b$ and $2 c$ which have expanded energy scales. It is also evident in Figures $2 b$ and $c$ that there is a shift in the kinetic energies of the Fe MVV peaks for both troilite and pyrite. These differences are extremely reproducible; however, it should be noted that a final calibration of the spectrometer has not been performed. Thus, while the observed peak shapes and shifts are reliable, absolute energies are not at this time. As a point of emphasis, the relative differences in peak intensity, shape, and energy (and not absolute energy) will be used to understand the thermal stability and surface chemistry of the iron sulfides.

Based on AES peak-to-peak (p-p) intensities, both samples have nominal stoichiometries. This assessment is complicated, however, by the fact that the peak shapes change from compound to compound. To remedy this problem, future quantitative work will utilize the integrated peak areas of $N(E)$ spectra rather than p-p intensity measurements of the $d N(E) / d E$ spectra. Examples of $N(E)$ spectra for the Fe MVV peaks are shown in Figure 3. These spectra have the advantage that they may be numerically fitted and integrated which makes the determination of total peak intensity much more precise.

A review of the electronic structure of the iron sulfide system is in progress. Specifically, this includes the details of the band structure, work function, and surface states of pyrite and the pyrrhotites. Motivated by increased interest in the iron sulfides as active 
solar cell materials, these data are becoming more readily available. When combined with a knowledge of the molecular structure of specific reactive species, these data will provide a basis for interpreting both the AES spectra and understanding the observed surface reaction. In addition these data will be useful in extending the results of the surface studies to experimentally inaccessible conditions.

Table 1 shows the results of a preliminary review of the literature on iron sulfide electronic structure. The predicted structure is based on the fact that the iron in pyrite and pyrrhotite is octahedrally coordinated by sulfur, and, thus, the $\mathrm{Fe} 3 \mathrm{~d}$ electrons are split into three $t_{2}$ and two $e_{z}$ levels. The predictions for both high and low spin occupation of these levels is shown in the table along with a very brief summary of the observed properties. Apparently both pyrite and trollite are semiconductors (consistent with the low spin configuration). The observed p-type semiconductor behavior is most likely due to $\mathrm{Fe}^{+3}$ defects. The pyrrhotites appear to be metallic in nature which is consistent with either the high or low spin configuration. This suggests that under reaction condition the catalyst which is a mixture of pyrite and pyrrhotite has both metallic and semiconductor characteristics. Both types of surfaces are known to be catalytic under appropriate conditions; however, one might suspect that the metallic surfaces would be more effective in dissociating molecular hydrogen than the semiconductor surface.

For the present these considerations are only speculative but are precisely the type of issue which will be addressed in this portion of the project. Further, it is important to point out two caveats which will be addressed in subsequent work. First, the choice between the high and low spin configuration, which determines the basic electronic structure, is determined by the strength of the octahedral field, and this is extremely sensitive to factors such as defects and defect density - both influenced by synthesis conditions. Second, is the even more fundamental issue of how many $\mathrm{Fe}$ and $\mathrm{S}$ atoms are required to constitute a bulk-like structure implicit in the above considerations. This may become an important issue since a $2 \mathrm{~nm}$ sized particle is approximately the size of a monoclinic pyrrhotite unit cell. Again, however, this is an issue whose merits and relevance will emerge in the course of the planned work. 
Kinetic Modeling of Ferric Sulfide Disproportionation

Ferric sulfide $\left(\mathrm{Fe}_{2} \mathrm{~S}_{3}\right)$ has been used as a precursor to synthesize mixtures of pyrite and pyrrhotite. At temperatures greater than $5^{\circ} \mathrm{C}$, ferric sulfide disproportionates to form pyrite $\left(\mathrm{FeS}_{2}\right)$, wunstoichiometric pyrrhotite $\left(\mathrm{FeS}_{x}\right)$, and elemental sulfur through the reaction:

$$
\mathrm{Fe}_{2} \mathrm{~S}_{3} \stackrel{\mathrm{k}_{\mathrm{t}}}{\rightarrow} \mathrm{FeS}_{2}+\mathrm{FeS}_{\mathrm{x}}+(1-\mathrm{x}) \mathrm{S}
$$
(A)
(Y)
(H)

We observed from the experimental data that the relative amounts of pyrite and pyrrhotite vary with the gas phase, the time and temperature of disproportionation. To account for this, an additional reversible reaction has been postulated:

$$
\begin{aligned}
& \mathrm{FeS}_{2} \stackrel{\mathrm{k}_{2}}{\leftrightarrow} \mathrm{FeS}_{\mathrm{x}}+(2-\mathrm{x}) \mathrm{S} \\
& \text { (Y) } \quad \mathbf{k}_{3} \quad(\mathrm{H})
\end{aligned}
$$

Here $k_{1}, k_{2}$ and $k_{3}$ are the reaction rate constants. The rate expressions for the above equations are written as:

$$
\begin{gathered}
\frac{d C_{\mathrm{A}}}{d t}=-k_{1} C_{\mathrm{A}} \\
\frac{d C_{Y}}{d t}=k_{1} C_{\mathrm{A}}-k_{2} C_{Y}+k_{3} C_{Y} \\
\frac{d C_{X}}{d t}=k_{1} C_{\mathrm{A}}-k_{2} C_{Y}+k_{3} C_{Y}
\end{gathered}
$$

where $C_{A}, C_{Y}, C_{H}$ are the concentrations of the three species. In evaluating the rate expressions, the amount of sulfur formed is assumed to be constant. The three nonhomogeneous linear differential equations have been solved using the method of undetermined coefficients and subject to the initial condition: at $t=0, C_{A}=C_{A O}$ and $C_{Y}=C_{H}$ $=0$. The expressions for $\mathrm{C}_{\mathrm{Y}}, \mathrm{C}_{\mathrm{H}}$, and $\mathrm{C}_{\mathrm{A}}$ are: 


$$
\begin{aligned}
& C_{Y}=\frac{2 k_{3}}{\left(k_{2}+k_{3}\right)} C_{\lambda O}+\frac{k_{1}\left(k_{2}-k_{3}\right) C_{10}}{\left(k_{2}+k_{3}\right)\left(k_{1}-k_{2}-k_{3}\right)} e^{-\left(k_{2}+k_{3}\right) t}+\left(\frac{2 k_{3}-k_{1}}{k_{1}-k_{2}-k_{3}}\right) C_{10} e^{-k_{1} t} \\
& C_{H}=\frac{2 k_{2}}{\left(k_{2}+k_{3}\right)} C_{A O}-\frac{k_{1}\left(k_{2}-k_{3}\right) C_{A O}}{\left(k_{2}+k_{3}\right)\left(k_{1}-k_{2}-k_{3}\right)} e^{-\left(k_{2}+k_{3}\right) t}+\left(\frac{2 k_{2}-k_{1}}{k_{1}-k_{2}-k_{3}}\right) C_{A O} e^{-k_{1} t} \\
& C_{\lambda}=C_{A 0^{-k_{3} t}}
\end{aligned}
$$

To obtain the kinetic parameters, the experimental data are being fitted to the mathematical model using the IMSL subroutine RNLIN. Using the mathematical model a variety of pyrite/pyrrhotite stoichiometries will be predicted as functions of time and temperature of disproportionation.

In order to obtain the kinetic data necessary for the evaluation of the model, a standardized synthesis scheme has been developed. This scheme allows the production of a wide range of pyrite/pyrrhotite ratios. A wet chemical analysis is performed on these products to determine their relative proportions. Shown in Figure 4 is a reaction flow chart summarizing the synthesis and evaluation procedure. Previous reports have described data obtained from hydrothermal runs. Computer analysis of these data fitted to the above model is underway. Current synthesis of mixed iron sulfides under hydrogen and nitrogen atmospheres is nearing completion. However, the reaction scheme cited above needs to be modified for reactions in a hydrogen atmosphere where $\mathrm{H}_{2} \mathrm{~S}$ is also a reaction product. Details of these experiments will be reported next quarter.

Currently, the Blind Canyon Coal (DECS-17) is being liquified without catalyst to establish baseline liquefaction behavior. Data from both a stirred-autoclave reactor as well as a shaken tubing-bomb reactor are presented in the section for Project II.6 entitled "Test Reactor for Catalytic Coal Liquefaction". Currently, most non-catalytic runs are completed and synthesis of specific pyrite/pyrrhotite catalysts is underway for use in the catalytic tests. Ratios of $0.5,1,3$ and 6 will be tested for their catalytic activity. Samples of these catalysts have been sent to DOE-PETC for testing. 
Table 1. Electronic Structure of Pyrite and Pyrrhotites.

PREDICTED STROCTURE

COIPOUND ION VALENCIES

Octabedral Splitting Fe 3d

(LOW Spin) (High Spin) OBSERVED PROPERTIE.

Troilite

FeS $\left(\mathrm{Fe}^{+2}\right)_{1}\left(\mathrm{Fe}^{+3}\right)_{0}\left(\mathrm{~S}^{-2}\right)_{1}$ Semiconductor Metal p-type/Fe ${ }^{+3}$ Defect:

Intermediate Pyrrhotites

$\mathrm{Fe}_{11} \mathrm{~S}_{12} \quad\left(\mathrm{Fe}^{+2}\right)_{9}\left(\mathrm{Fe}^{+3}\right)_{2}\left(\mathrm{~S}^{-2}\right)_{12}$ Metal Metal Magnetic

$\mathrm{Fe}_{10} \mathrm{~S}_{11}\left(\mathrm{Fe}^{+2}\right)_{8}\left(\mathrm{Fe}^{+3}\right)_{2}\left(\mathrm{~S}^{-2}\right)_{11}$ Metal Metal Magnetic

$\mathrm{Fe}_{9} \mathrm{~S}_{10}\left(\mathrm{Fe}^{+2}\right)_{7}\left(\mathrm{Fe}^{+3}\right)_{2}\left(\mathrm{~S}^{-2}\right)_{10}$ Metal Metal Magnetic

Monoclinic Pyrrhotite

$\mathrm{Fe}_{7} \mathrm{~S}_{8} \quad\left(\mathrm{Fe}^{+2}\right)_{2}\left(\mathrm{Fe}^{+3}\right)_{2}\left(\mathrm{~S}^{-2}\right)_{8} \quad$ Metal $\quad$ Metal Magnetic

Pyrite/Maracasite

$\mathrm{FeS}_{2} \quad\left(\mathrm{Fe}^{+2}\right)_{1}\left(\mathrm{Fe}^{+3}\right)_{0}\left(\mathrm{~S}_{2}\right)^{-2} \quad$ Semiconductor Metal $\quad \mathrm{p}$-Type $/ \mathrm{Fe}^{+3}$ Defect 
FIGURE \& TABLE CAPTIONS

Figure 1. Schematic diagram of the surface analysis systems.

Figure 2. AES derivative spectra ( $d N(E) / d E$ ) for trollite and pyrite. a) The spectral region from $0-160$ eV showing the $F e \mathrm{MVV}$ and S LVV peaks; b) The $25-60$ eV region showing the Fe MVV pealis; and c) The 110-160 region showing the S LVV peaks.

Figure 3. $N(E)$ spectra for the $F e$ MVV peaks of troilite and pyrite.

Figure 4. Experimental scheme for iron sulfide disproportionation reaction and analysis.

Table 1: Electronic Structure of Pyrite and Pyrrhotites. 


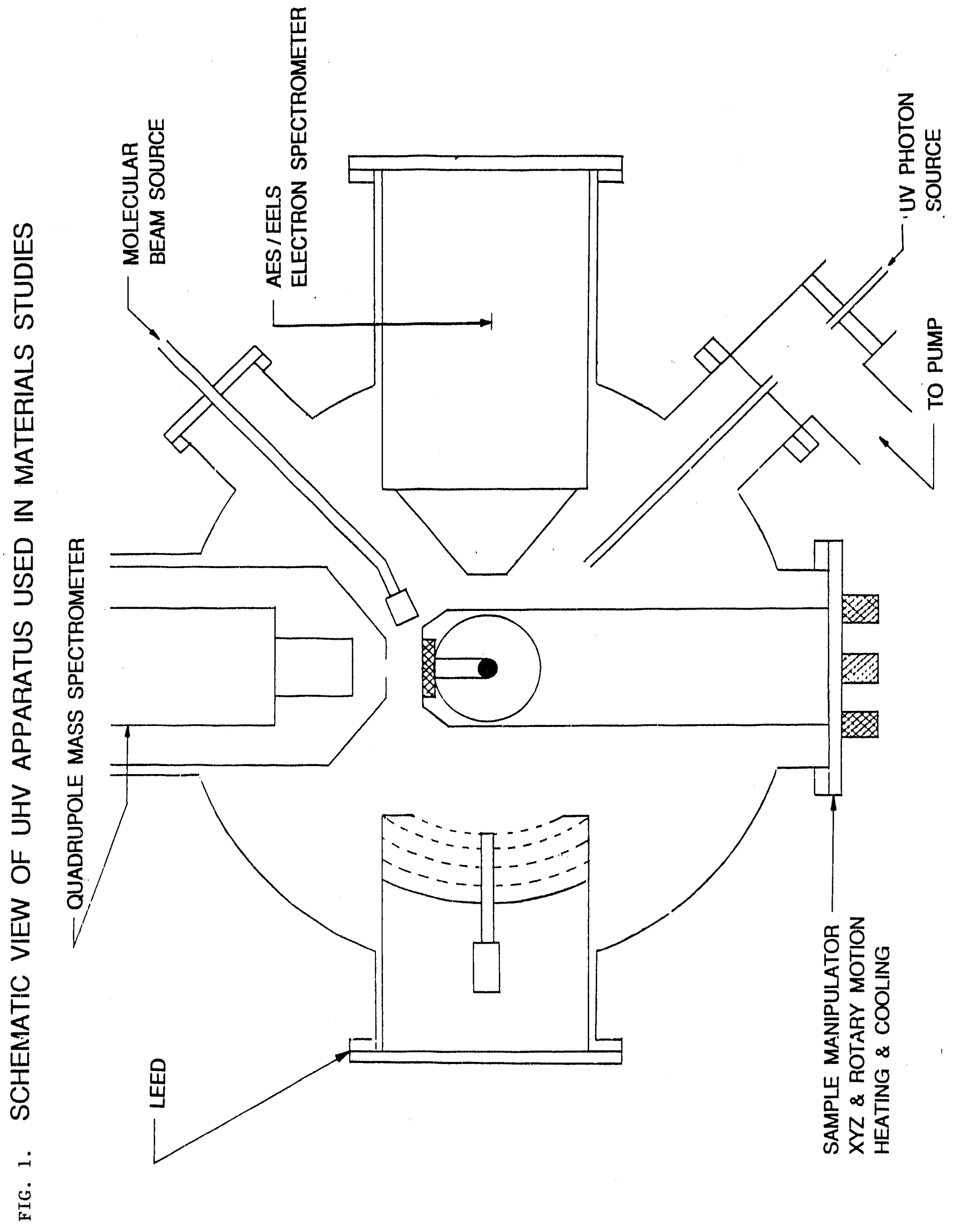




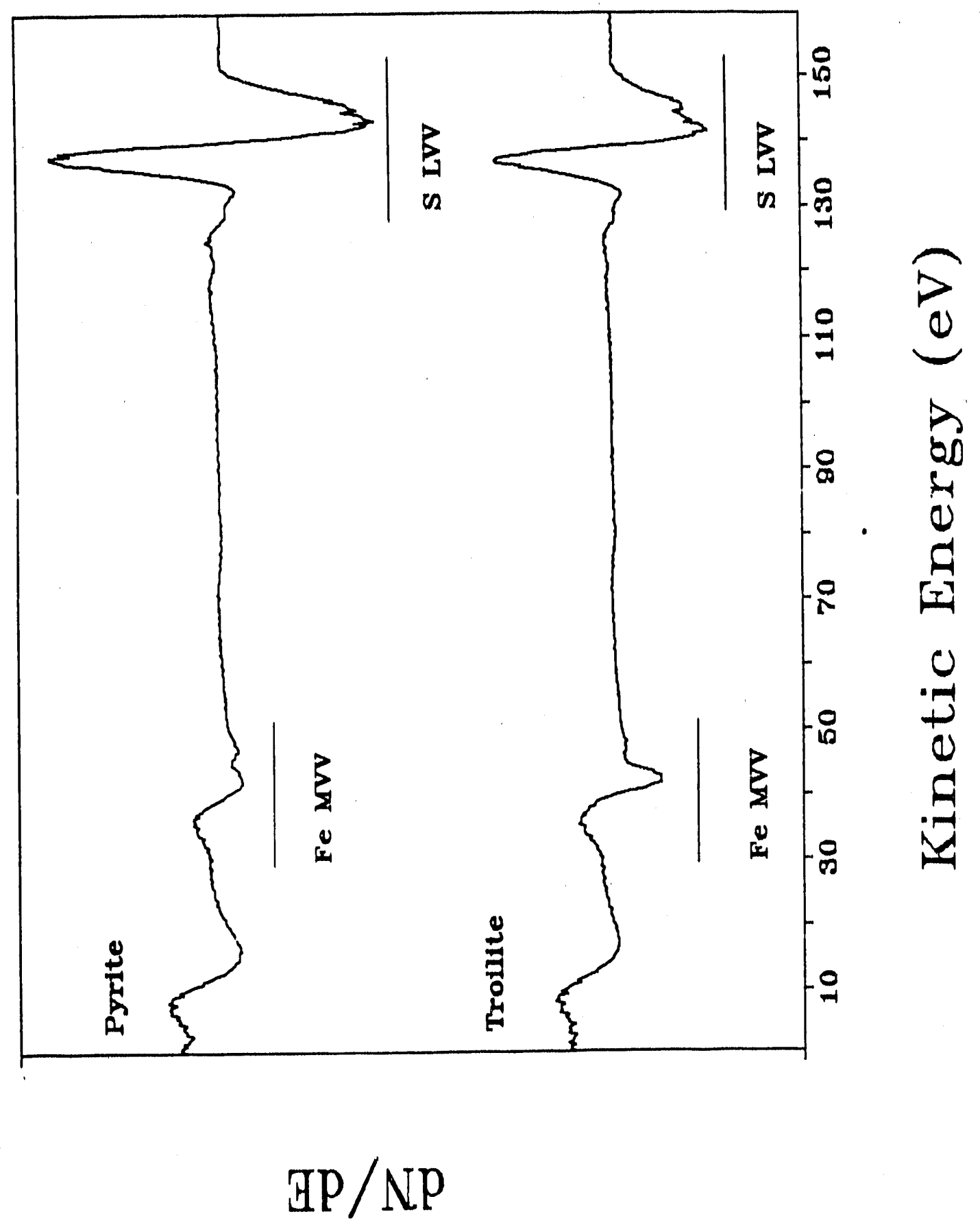




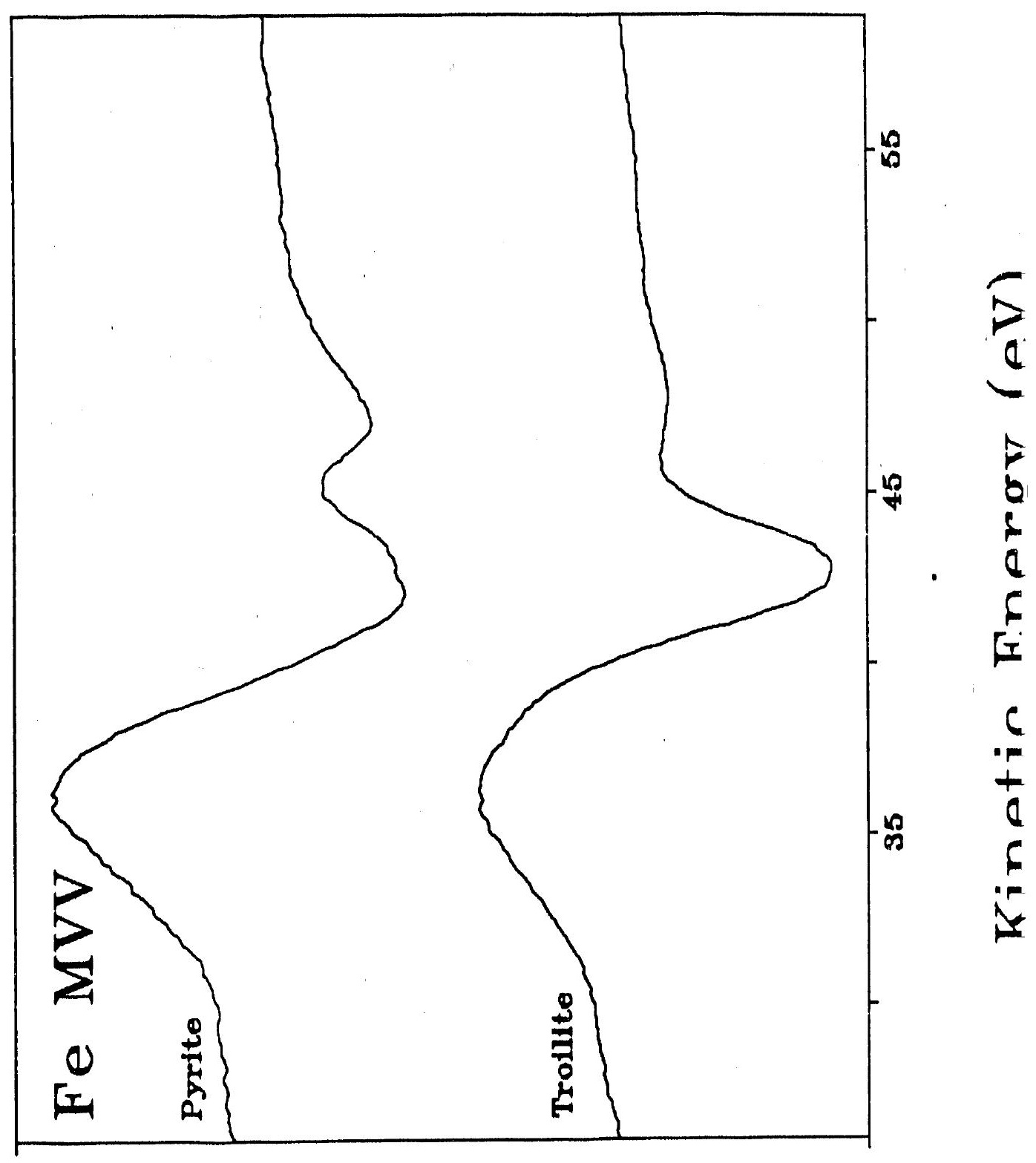

GP/N.P 


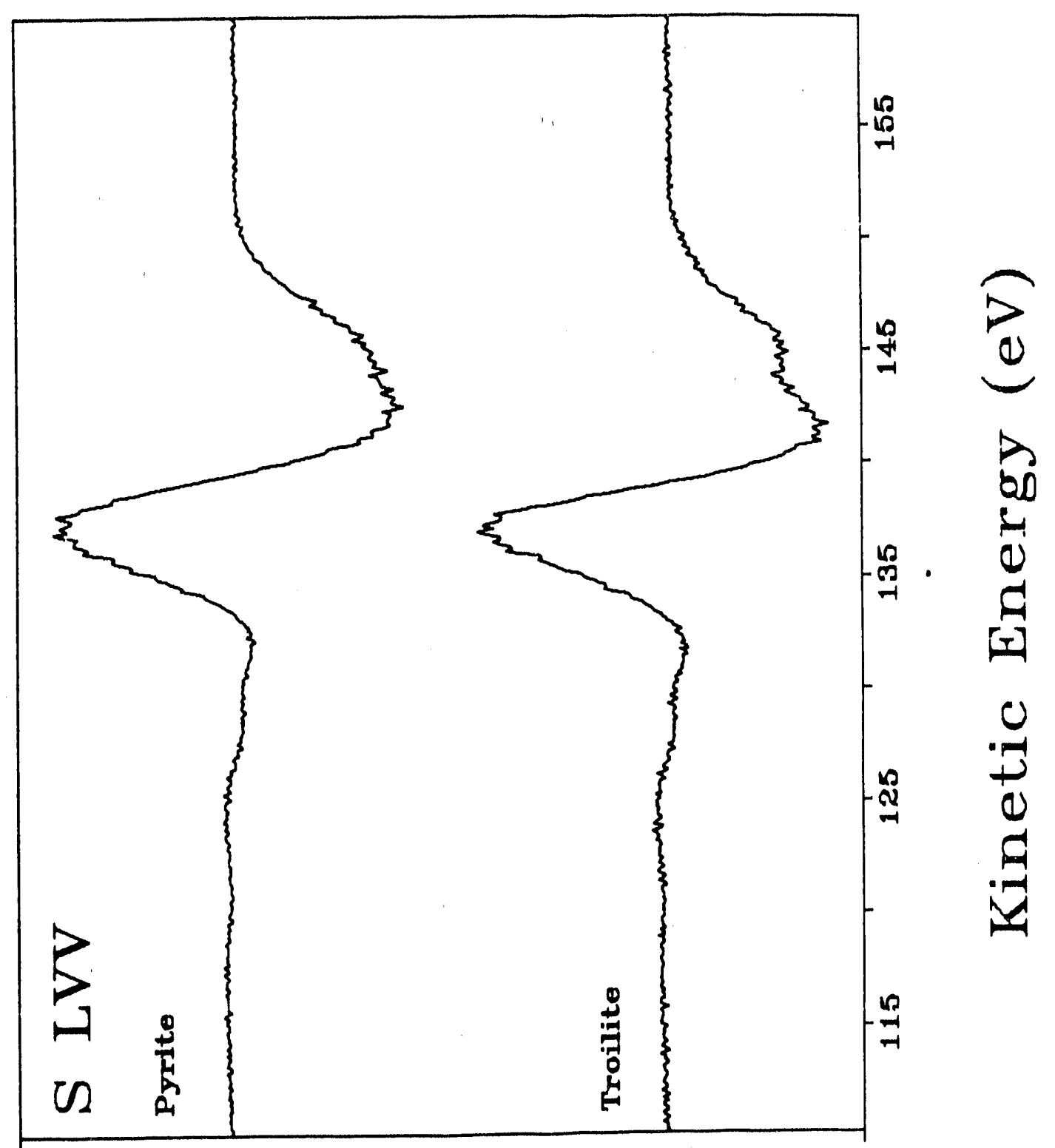

GP/NP 


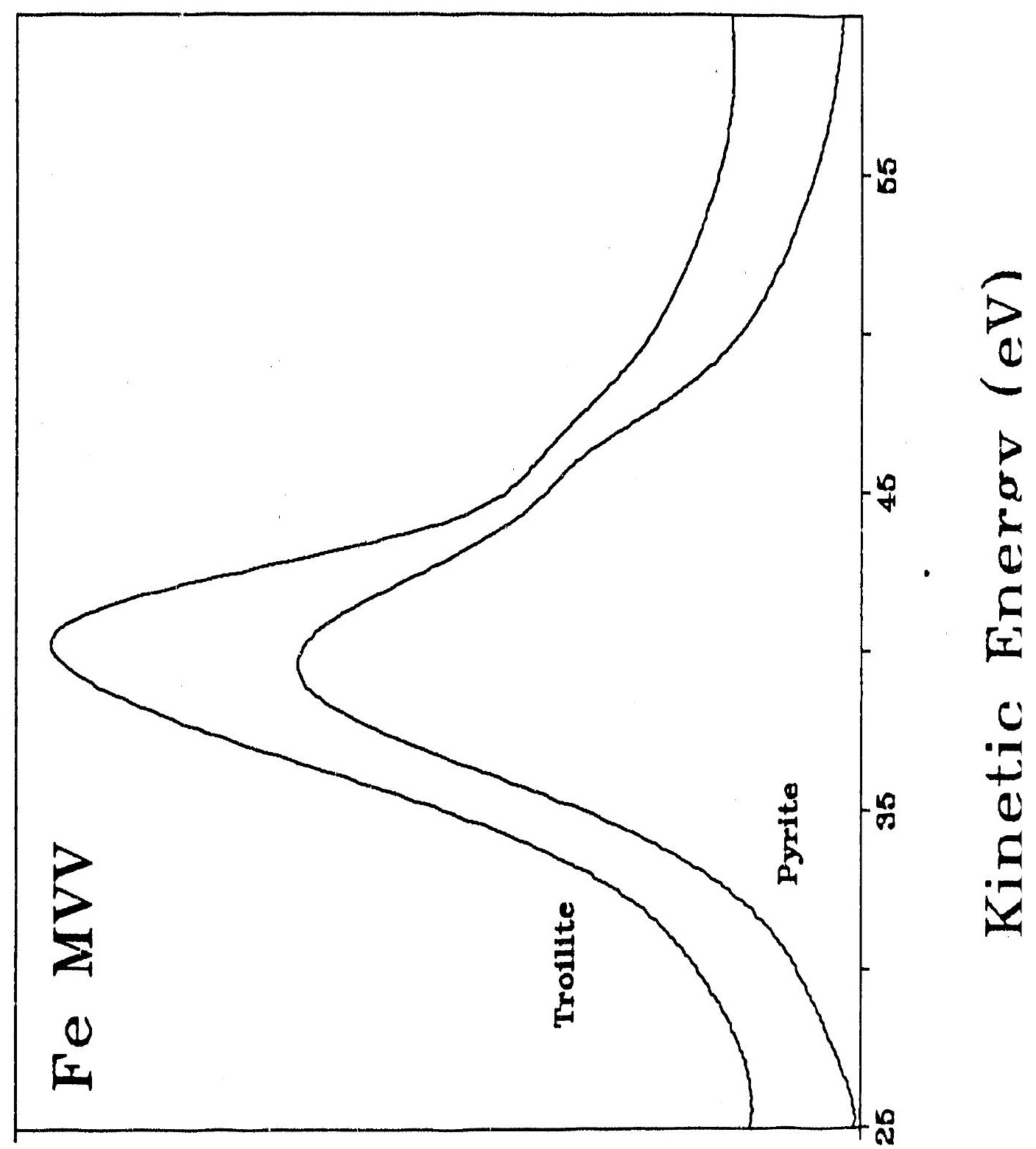

(B) $\mathrm{N}$ 


\section{FIG. 4. Experlmental Scheme for Iron Sulflde Disproportionatlon Reactlon andAnalysls}

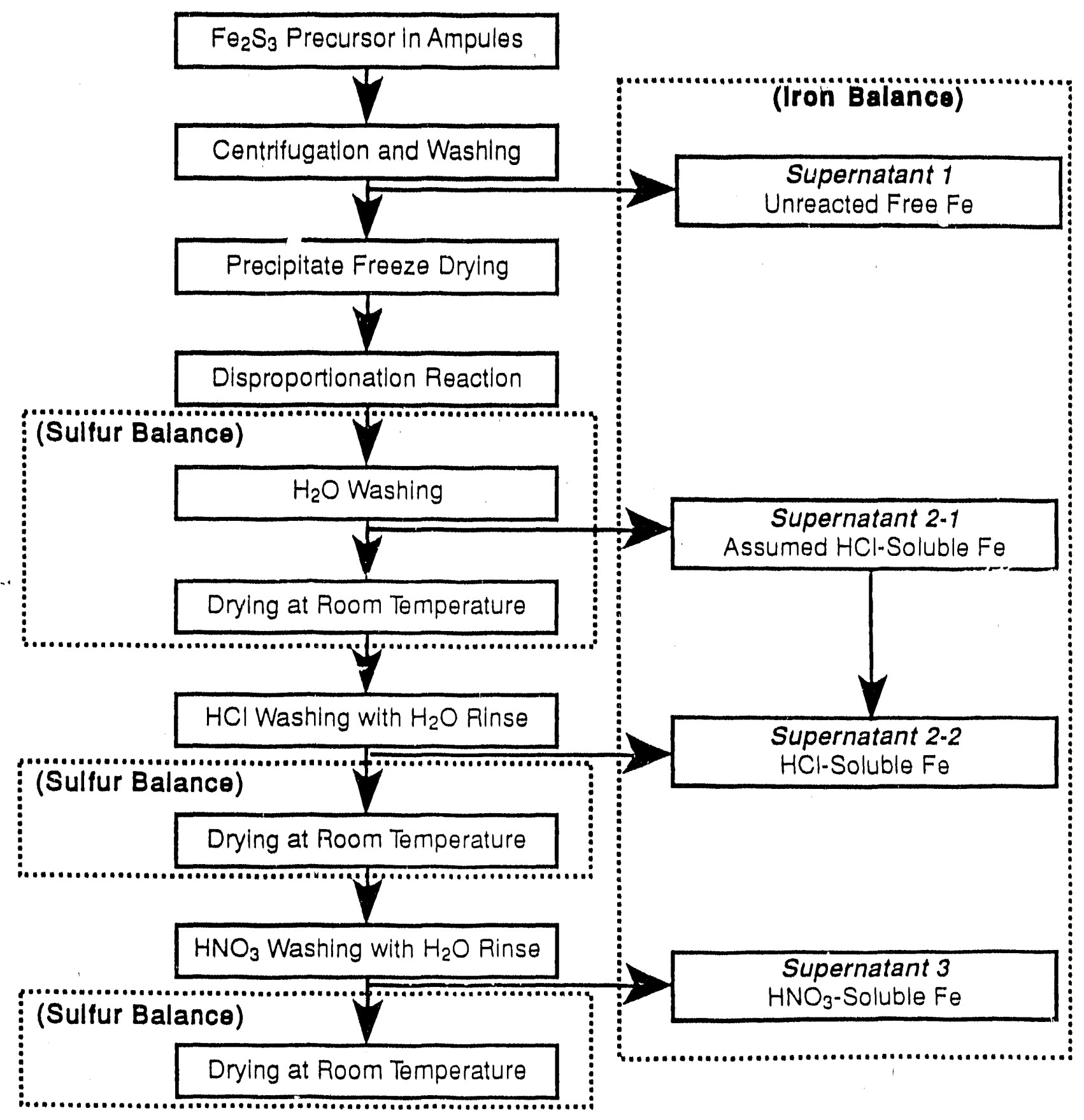




\title{
Project I.8 Laser Pyrolysis Production of Ultrafine Particle Catalysts for Coal Liquefaction
}

\author{
P.C. Eklund \\ Center for Applled Energy Research \\ University of Kentucky
}

\section{METHOD OF FINE PARTICLE SYNTHESIS}

$\mathrm{A} \mathrm{CO}_{2}$ laser pyrolysis system has been built for the synthesis of ultrafine iron carbide particles by the reaction of $\mathrm{Fe}(\mathrm{CO})$, and $\mathrm{C}_{2} \mathrm{H}_{4}$. By varying reaction parameters, we have been able to produce three pure phase nanosize particles, alpha- $\mathrm{Fe}, \mathrm{Fe}_{3} \mathrm{C}$ and $\mathrm{Fe}_{4} \mathrm{C}_{3}$ with sizes ranging from $6 \mathrm{~nm}$ to $30 \mathrm{~nm}$. The reaction parameters including chamber pressure, laser Intensity and focusing, $\mathrm{C}_{2} \mathrm{H}_{4}$ flow rate, and reactant gas nozzle diameter have been investigated to understand their influence on the particle properties. In particular, $\mathrm{Fe}_{7} \mathrm{C}_{3}$ has been produced for the first time in its pure form using this technique. We have observed that $\mathrm{Fe}_{7} \mathrm{C}_{3}$ phase particles can be made under low laser power, fast flow speed of reactant gas and short residence time in the reaction zone. A transformation to $\mathrm{Fe}_{3} \mathrm{C}$ and possibly carbon coated alpha-Fe is noticed with excessive laser heating. In this system, we have not seen the formation of $\mathrm{Fe}_{3} \mathrm{C}_{2}$ in the reaction with any set of reaction parameters between the ones for producing $\mathrm{Fe}_{3} \mathrm{C}$ and $\mathrm{Fe}_{7} \mathrm{C}_{3}$. Production rates as high as 1.5 gram/hour have been achieved in the current system. Furthermore, by mixing $\mathrm{H}_{2} \mathrm{~S}$ into the reactant gas stream, we have recently generated nanoscale $(-10 \mathrm{~nm}) \mathrm{Fe}$-sulfide particles identified with pyrrhotite $\left(\mathrm{Fe}_{1 \cdot \times} \mathrm{S}\right.$, $0<x<0,125)$. Further investigation is underway to probe the reaction parameters in producing these sulfide particles with different phases and sizes. No coal liquefaction studies have $\mathrm{Fe}_{1 \cdot \mathrm{x}} \mathrm{S}$ particles to date.

\section{REFERENCES}

a. Haggerty, J.S., Cannon, W.R., 1981, in Laser-Induced Chemical Processes (Ed. II Steinfeld), Plenum Press, New York, NY, USA.

b. Fiato, R.A. et al., 1987, US pat. 4, 637, 753.

c. Hager, G.T., Bi, X.X., Derbyshire, F.J., Eklund, P.C., Stencel, J.M., American Chemical Society, Div, of Fuel Chemistry, Preprints, 1991, 36(4) 1900-1908.

\section{INFORMATION ABOUT CATALYST AVAILABILITY}

The catalysts are currently being produced in 5 gram quantities for testing.

\section{CHARACTERIZATION METHODS}

The iron carbide particles have been characterized by TEM (Transmission Electron 
Microscope), SEM-Microprobe, XRD (X-Ray Diffraction), Mossbauer spectroscopy and Raman scattering. Combined study by TEM and XRD show that the carbide particles have a crystalline structure in the particle core and a carbon coating on the surface. The existence of graphitic carbon is further observed in Raman scattering experiments. Mossbauer studies have been employed to identify the magnetic phase change of particles to the superparamagnetic state.

\section{PRELIMINARY RESULTS OF CATALYST TESTING}

Recent work in coal liquefaction has focused on the $\mathrm{Fe}_{7} \mathrm{C}_{3}$ phase. Activation procedure - The Iron carbide particles were activated prior to addition to the reactors.

Coal liquefaction - Iron carbides produced were tested as coal liquefaction catalyst precursors in 15-minute, vertically-shaken microautoclave experiments.

Experimental - A sub-200 mesh subbituminous Wyodak coal was used in the experiments, and was dried before the reactions. Three grams of coal were used with five grams of tetralin as the hydrogen donor solvent. The iron carbide catalyst precursor was added at $1 \mathrm{wt} \%$ (total Fe as metal) of the coal, as loaded to the reactor. When used, DMUS was added in an amount necessary for $120 \%$ stoichiometric conversion of the iron to pyrite. As shown in the attached figure, conversion experiments were run at about $330^{\circ} \mathrm{C}$ to $440^{\circ} \mathrm{C}$. The reactors were charged to 800 psig hydrogen (cold pressure).

Gas samples from the batch microautoclave experiments were analyzed for mole percentages of $\mathrm{H}_{2}, \mathrm{CO}, \mathrm{CO}_{2}, \mathrm{H}_{2} \mathrm{~S}$ and $\mathrm{C}_{1}-\mathrm{C}_{4}$ by gas chromatography. The quantity of each gas produced was calculated using the ideal gas law, the mole percent in the gas sample, and the postreaction vessel temperature and pressure.

Soxhlet extraction was utilized to determine the conversion yield of the liquefaction products. The benzene solubles were further classified as pentane insoluble (asphaltenes) and soluble (oil fraction). The benzene insoluble material was extracted with pyridine, the soluble portion being classified as preasphaltene and the pyridine insolubles as IOM.

Results - Preliminary experiments using a variety of catalyst precursors indicate that the coal liquefaction followed a typical subbituminous pathway. While beneficial to coal conversion, the iron carbide experiments also followed this same pathway. As shown in the figure, $\mathrm{Fe}_{7} \mathrm{C}_{3}$ gave good liquefaction results when used with DMDS, giving 7-9 percent conversion enhancement (total pyridine solubles) in the $360-390^{\circ} \mathrm{C}$ range. Conversion was tested with an iron naphthenate, and results were similar to the carbide. Early testing with a commercially available $\mathrm{Fe}_{2} \mathrm{O}_{3}$ at the same catalyst loading resulted in lower conversion.

Results with model compounds - no experimental work has been done to date using model compounds. 


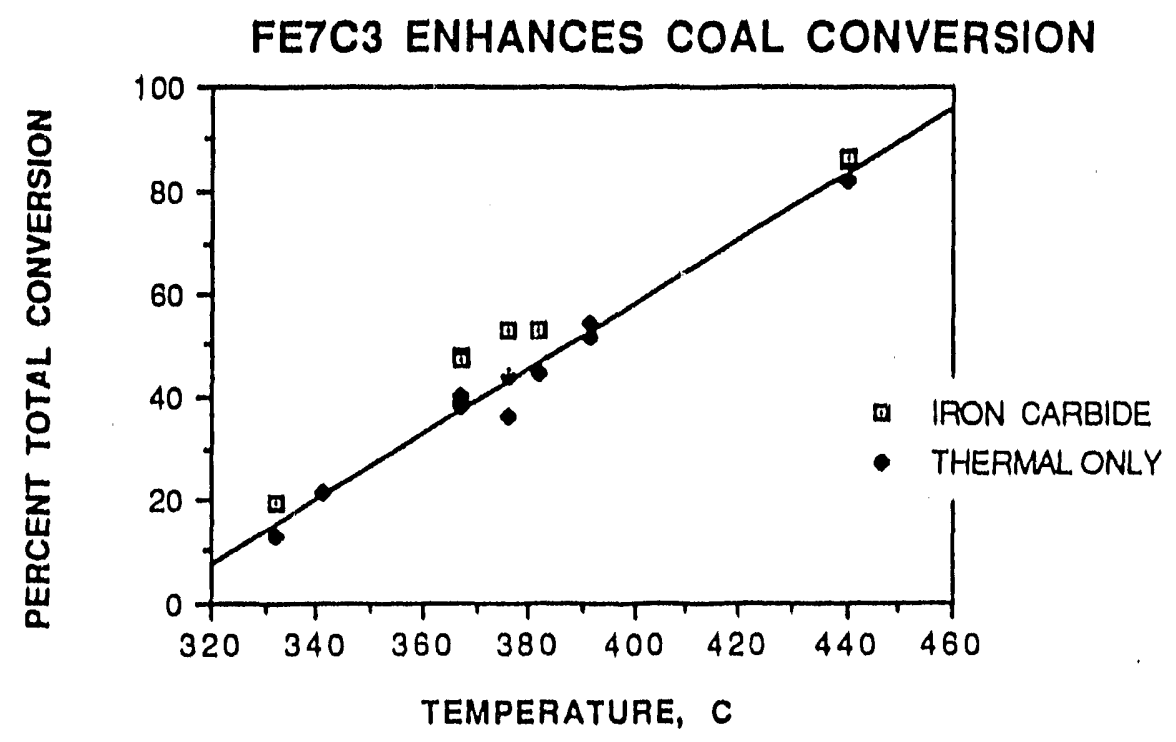




\section{Task II}

Exploratory Research on Coal Conversion

Program Ccordinators: Henk Meuzelaar and Mohindar Seehra 


\title{
Project II.1 Coal Structure/Liquefaction Yield Correlation by Means of Advanced NMR Technique
}

\author{
R.J. Pugmire \\ University of Utah
}

\begin{abstract}
During the last six month period our efforts have been on the following two areas each described below :

1) New methods to obtain chemical shift tensors in model compounds and coals

2) A comprehensive NMR study of anthracite coals using both magic angle spinning and static measurements.
\end{abstract}

In addition we have continued providing NMR analyses on the products of liquefaction processes in support of other participants in CFFLS.

\section{Measurement of Chemical Shift Tensors in Model Compounds and Coals}

In past reports we have discussed the acquisition of equipment necessary to do the dynamic angle spinning (DAS) experiment [1,2]. After much interaction with the manufacturer we believe that we have a working DAS probe. A new Chemagnetics CMX-100 console is currently being installed and the implementation of the DAS experiment is one of the top priorities.

Recent efforts in our laboratory on the combination of slow spinning at the magic angle [3] with selective excitation [4] and dipolar dephasing [5] techniques have shown promise in the measurement of the chemical shift tensors in fairly complex model aromatic compounds. Side band intensity patterns can then be used to determine the principal values of the chemical shift tensor [6]. An example of the results obtained by the combination of these techniques on 1,2,3-trimethoxybenzene is shown in Figure 1. Figure 1a is the normal fast MAS $\left(\omega_{\mathrm{r}}=6 \mathrm{KHz}\right)$ spectrum, showing four different aromatic carbons and two methoxy carbons. Figure $1 \mathrm{~b}$ is a slow spinning $\left(\omega_{\mathrm{r}}=1.7 \mathrm{Khz}\right)$ spectrum of the same compound. With the use of the standard dipolar dephasing with a dephasing period of $70 \mu \mathrm{s}$, only the sideband from the unprotonated and rotating methyl groups remains, as shown in Figure 1c. Finally one sideband pattern group can be selectively excited resulting in the individual patterns shown in Figures $1 \mathrm{~d}$ and $1 \mathrm{e}$, for $\mathrm{C}_{1,3}$ and $\mathrm{C}_{2}$, respectively. By fitting the intensity pattern of the sidebands one retrieves the principal values of the chemical shift tensor given in Table 1. For comparison, the principal values obtained on 1,2,3,- trimethoxybenzene via a single 
crystal experiment [7] (which gives the most accurate solid state results) are also listed. Agreement between the two methods is excellent except for $\sigma_{11}$ and $\sigma_{22}$ of $\mathrm{C}_{2}$; efforts are underway to duplicate this experiment and resolve this dilemma.

Preliminary results on the application of these techniques to a coal sample are given in Figure 2 and Table 2. The coal, PSOC-867, is an anthracite coal, and therefore, the lineshape should be due to both protonated and bridgehead carbon shift patterns. Figure 2a is the slow spinning pattern obtained using a normal CP sequence. With a $50 \mu \mathrm{s}$ dephasing time the sideband pattern changes to that given in Figure $2 b$ and should be due to the unprotonated bridgehead carbons. The difference of the two spectra, shown in Figure 2c, should therefore be due to the protonated carbons. The tensor components obtained are given in Table 2 along with the tensor components obtained from the fit of a static powder pattern [8]. In this case the agreement is not as good as for the model compound test case, but it is acceptable considering the inaccuracies expected in both sets of results. Currently these techniques are being applied to a lower rank coal.

\section{NMR Study of Anthracite Coals}

A set of nine anthracite and semi-anthracite coals from the Penn State Coal Sample Bank have been investigated by ${ }^{13} \mathrm{C}$ CP/MAS and static NMR techniques, From a set of variable contact time $\mathrm{CP}$ runs and a set of dipolar dephasing experiments a description of an average aromatic cluster is obtained [9]. These results are shown in Table 3. The static CP measurements are used to obtain the tensor components for the protonated and condensed aromatic carbons; the fitting of these static powder patterns is still progressing and the few results obtained to date are shown in Table 4. It should be noted that both MAS and static results provide aromatic $\mathrm{H} / \mathrm{C}$ ratios in good agreement with the total $\mathrm{H} / \mathrm{C}$ ratio from elemental analysis on the anthracite coals (where aliphatic contents is negligible.)

In the analysis of the variable contact time data it was noticed that the model used to describe the increase and subsequent decay of magnetization in bituminous coals [10] worked well for the semianthracites but could not satisfactorily fit the data on anthracite coals. The anthracite coals required the addition of a third term to properly describe the build up of aromatic intensity. A typical fit of the experimental data from an anthracite coal under the two different models, VCLG and 3TCH, are shown in Figure 3. This 3TCH model uses three terms, one Gaussian and two Lorentizian, to describe the increase in intensity as a function of contact time, along with one relaxation time. A tentative explanation for the need of this third term can be made as follows. The Gaussian term accounts for approximately half of the protonated carbons along with the outer quaternary carbons, consistent with the current theories on a two stage polarization transfer [11]; these two terms are present in the models used on bituminous coals. However, in the anthracite coals there exists a large percentage of bridgehead carbons that are spatially further from the protons, the so-called inner quaternary carbons, due to the larger cluster size than in the lower rank coals. It is necessary to add a second longer Lorentzian term to describe the magnetization transfer to these inner 
carbons. Also, since the aliphatic substitution of the aromatic clusters is low, the majority of the protons are aromatic and therefore only one $T_{1 p}$ is needed to describe the relaxation process. Table 5 summarizes the time constants and the percentage of the spins for each of these terms. We are currently working on correlating these percentages with cluster sizes to obtain more details about the aromatic structure.

A sechor noservation that can be made from the NMR data is that the average number of aromatic irtorls per cluster (AC/CI) of the semi-anthracite is essentially the same as that in the low villatile bituminous coals, about 20 . In most cases of anthracite there is an increase in $\mathrm{AC} / \mathrm{CI}$ of about a factor of two. However in PSOC-867, the coal with the lowest atomic $\mathrm{H} / \mathrm{C}$ ratio $(0.13)$, there is a dramatic increase in the cluster size to 350 . It should be noted that the method of measurement leads to larger errors as the ciuster size increases and the result of 350 aromatic carbons per cluster has errors of about \pm 50 .

The analysis of static powder patterns has been more difficult as there are many problems with lineshapes possibly due to relaxation and orientational dependence on cross polarization process. Therefore the amount of tensorial data available to date is limited, and efforts are underway to better understand the factors that influence the lineshape.

\section{REFERENCES}

1. Bax, A.; Szeverenyi, N. M.; Maciel, G. E. J. Magn. Reson. 55, 494 (1983).

2. Nakai, T.; McDowell, C. A. J. Magn. Reson. 93, 618 (1991).

3. $\quad$ Marieq, M. M.; Waugh, J.S. J.Chem. Phys. 70,3300 (1979).

4. Tekely, P.; Brondeau, J.; Elbayed, K.; Retournard, A.; Canet, D. J. Magn. Reson. $\underline{80}$ 509 (1988). Caravatti, P.; Bodenhausen, G.; Ernst, R.R. J. Magn. Reson. 55, 88, (1983).

5. Opella, S.J.; Frey, M. H. J. Am. Chem. Soc. 101, 5854 (1979).

6. Herzfeld, J.; Berger, A. E. J. Chem. Phys. 73, 6021 (1980).

7. Carter, C.M.; Facelli, J. C.; Alderman, D. W.; Grant, D. M.; Dalley, N. K.; Wilson, B. E. J. Chem. Soc., Faraday Trans. 1, 84, 3673 (1988).

8. Sethi, N. K.; Pugmire, R. J.; Facelli, J. C.; Grant, D. M. Anal. Chem. 60, 1574 (1988).

9. Solum, M. S.; Pugmire, R. J.; Grant, D. M. Energy \& Fuel $\underline{3}, 187$ (1989).

10. Orendt, A. M.; Solum, M. S.; Sethi, N. K.; Pugmire, R. J.; and Grant, D. M. Advances in Coal Spectroscopy, H. L. C. Meuzelaar (Ed.), in press, 1991.

11. Muller, L.; Kumar, A.; Baumann, T.;Ernst, R. R. Phys. Rev. Lett. 32, 1402 (1974). Wu, X.; Zhang, S.; Wu, X. Phys. Rev. B. 37, 98:27 (1988). 


\title{
Project II.2 Mass Spectrometric Analysis and Modeling of Coal Liquefaction Processes
}

\author{
H.L.C. Meuzelaar and H. Huai \\ University of Utah
}

\section{On-Line GC/MS Monitoring of Model Compound Conversion Reactions at High Pressures}

As described in the last semi-annual report, an on-line GC/MS monitoring system for coal conversion processes under high pressure conditions has been designed and constructed in collaboration with $\mathrm{Dr}$. Shabtai's group.

Typical coal liquid upgrading reactions such as: hydrogenation, hydrodeoxygenation, hydrodesulfurization and hydrodenitrogenation have been studied under thermal and catalytic reaction conditions using suitable model compounds. The experimental conditions used are listed in Table 1. A schematic representation of the experimental apparatus can be found elsewhere [1].

Figure 1 shows the total ion chromatograms of dibenzyl ether and its hydrodeoxygenation reaction products under thermal as well as catalytic conditions at different reactor temperatures. As expected, the catalytically controlled reaction proceeds at markedly lower temperatures than the thermally controlled reaction. Figure 2 shows the kinetic profiles of dibenzyl ether as well as its hydrodeoxygenation products under thermal and catalytic conditions at 340 and $300^{\circ} \mathrm{C}$, respectively.

Figures 3 and 4 demonstrate the total ion chromatograms and kinetic profiles of a mixture of model compounds (diphenylsulfide/indole/biphenyl ether) as well as their respective reaction products under catalytic reaction conditions at $300^{\circ} \mathrm{C}$. Observed relative reaction rates are: diphenyl sulfide $>$ indole $>>$ biphenyl ether. Biphenyl ether is almost inert under these experimental conditions.

The primary significance of the novel on-line analysis lies (a) in the tremendous time savings (complete kinetic profiles can be obtained from a single 1-2 hour experiment rather than from a suite of consecutive experiments carried over a period of several weeks) and (b) in the possibility of identifying reactive products or intermediates which tend to be lost during conventional sampling, storage or analysis operations (e.g. as a result of exposure to air). 
2. On-Line GC/MS Monitoring During High Pressure Upgrading of Coal-Derived Liquids

Coal-derived liquids generally consist of complex mixtures of aliphatic, aromatic and heterocyclic compounds, often containing thousands of individual components.

Due to the extreme complexity of coal-derived liquids and the short retention time requirements of the on-line experiment, use of a 1 meter transfer line GC column (TLGC) under isothermal conditions, e.g. as used for the model compound studies, cannot satisfy the separation requirements. Consequently, a trmperature programmable TLGC technique has been developed in our laboratory. The short (1-2 $\mathrm{m}$ long) fused silica capillary column can be heated at a rate of $0-40 \mathrm{~K} / \mathrm{s}$ up to a final temperature of $300 \mathrm{C}$ and can be air cooled within 2-3 minutes. Rapid programmed heating of the transfer line GC column greatly increases the range of compounds which can be separated in a single run while only mildly increasing the run time of a single sample. According to preliminary test results, repetitive sampling intervals can be as short as 5-10 minutes.

Typically, raw coal-derived liquids are lower in hydrogen content and higher in heteroatomic $(\mathrm{O}, \mathrm{N}, \mathrm{S})$ content than petroleum crudes. This implies that upgrading coal-derived liquids involves both hydrogen addition and removal of nitrogen-, oxygen-, and sulfurcontaining functional groups as well as organometallic compounds. Different types of feed require different experimental conditions as well as catalysts. In our experiments, we are focusing on hydrode(hetero)genation reactions. Consequently, raw coal-derived liquids to be studied are prefractioned by open column elution chromatography in order to separate the non-polar from the polar (heteroatom-rich) fraction. The polar fraction is then used for hydrode(oxygen)(nitrogen)(sulfuriz)ation experiments. At the time of writing, the first on-line TLGC/MS monitoring experiments of low temperature hydrogenation reactions in polar subfractions of coal-derived liquids are underway in collaboration with Dr. Shabtai's group.

\section{REFERENCES}

1. Huai, H.; Meuzelaar, H.L.C.; Tsai, C.H.; and Shabtai, J.S. "Development of Fully Integrated Coal Liquefaction/Characterization Techniques," Proceedings of the 1991 DOE Contractor/Reviewers. Meeting, Pittsburgh, PA, September 3-5, 1991 (in press). 

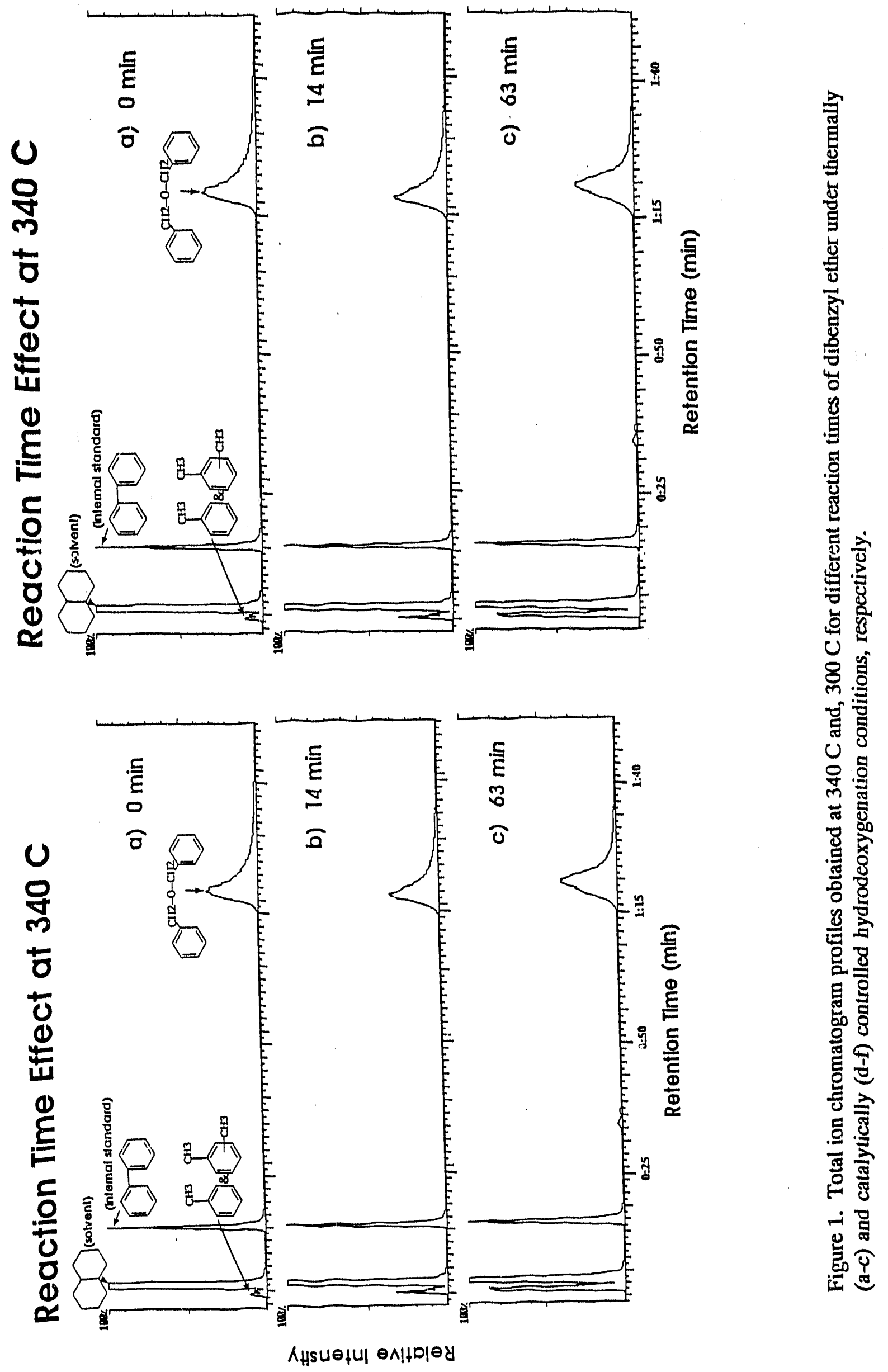


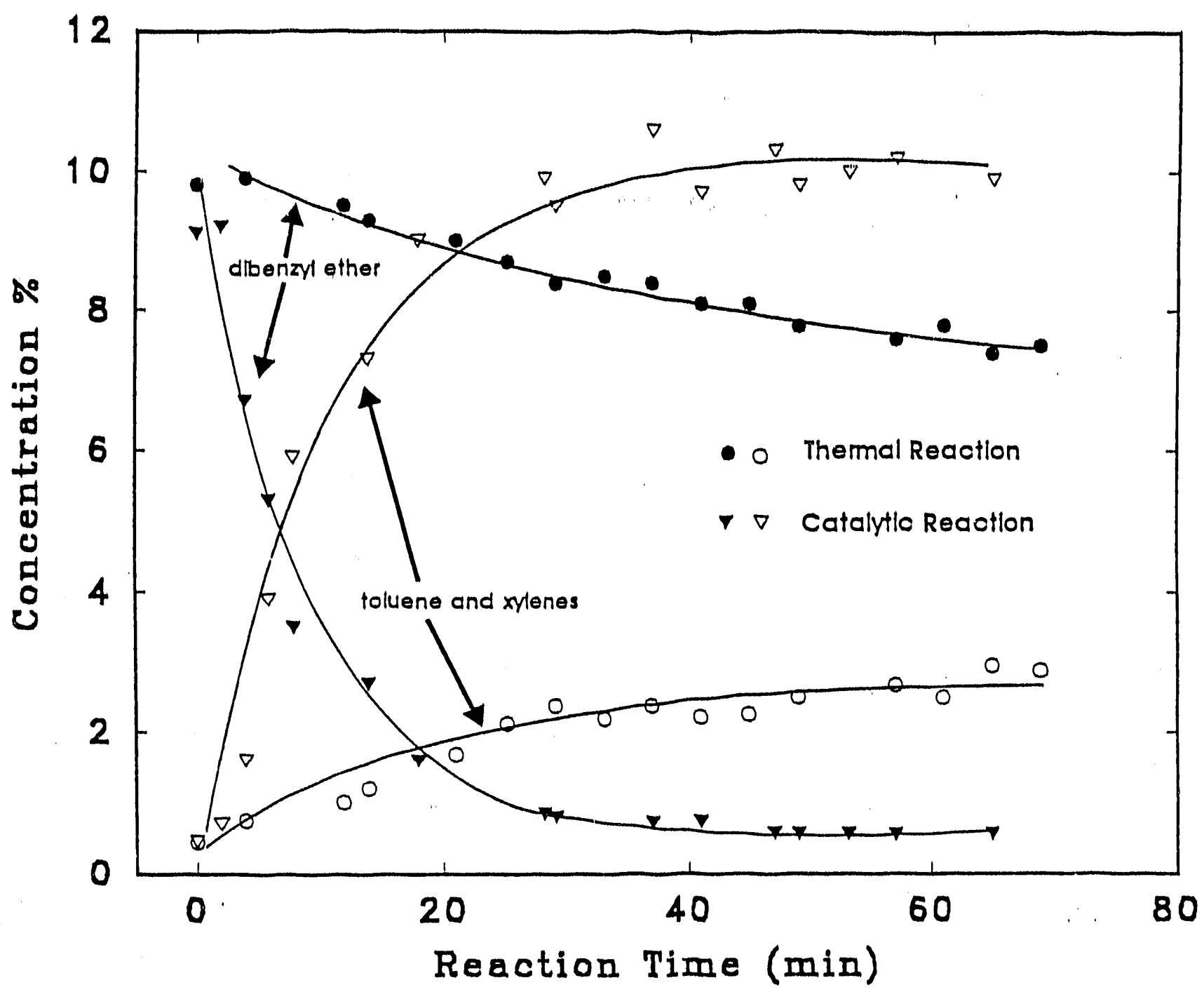

Figure 2. Kinetic profiles of dibenzylether conversion $(\bullet, \nabla)$ and toluene and xylenes formation $(0, \nabla)$ under thermally $(0,0)$ and catalytically $(\nabla, \nabla)$ controlled conditions. 


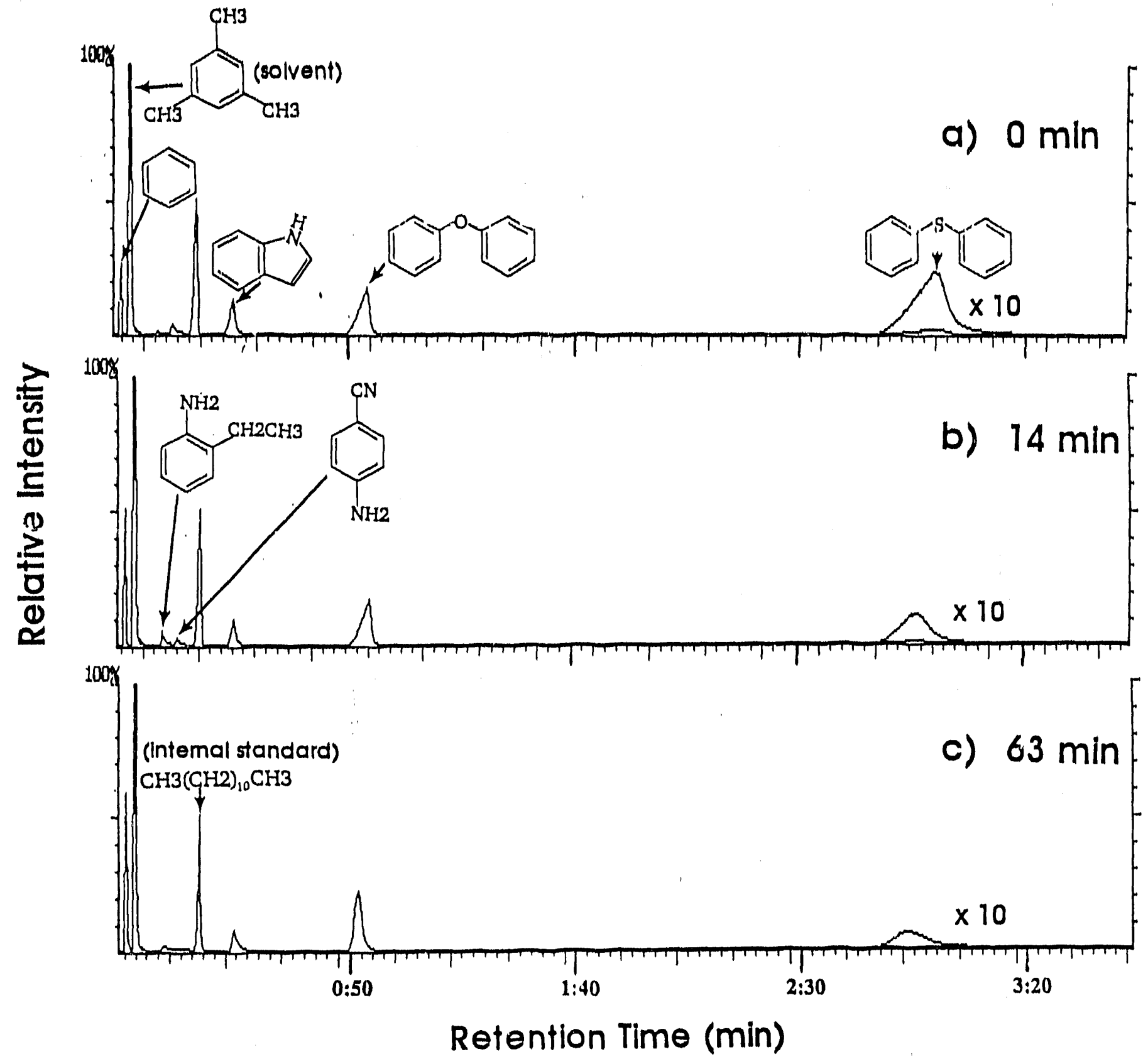

Figure 3. Total ion chromatogram profile obtained at $300 \mathrm{C}$, for different reaction times of diphenyl ether, indole and diphenyl sulfide under catalytically controlled hydrode(oxygen)(nitrogen)(sulfuriz)ation conditions. 


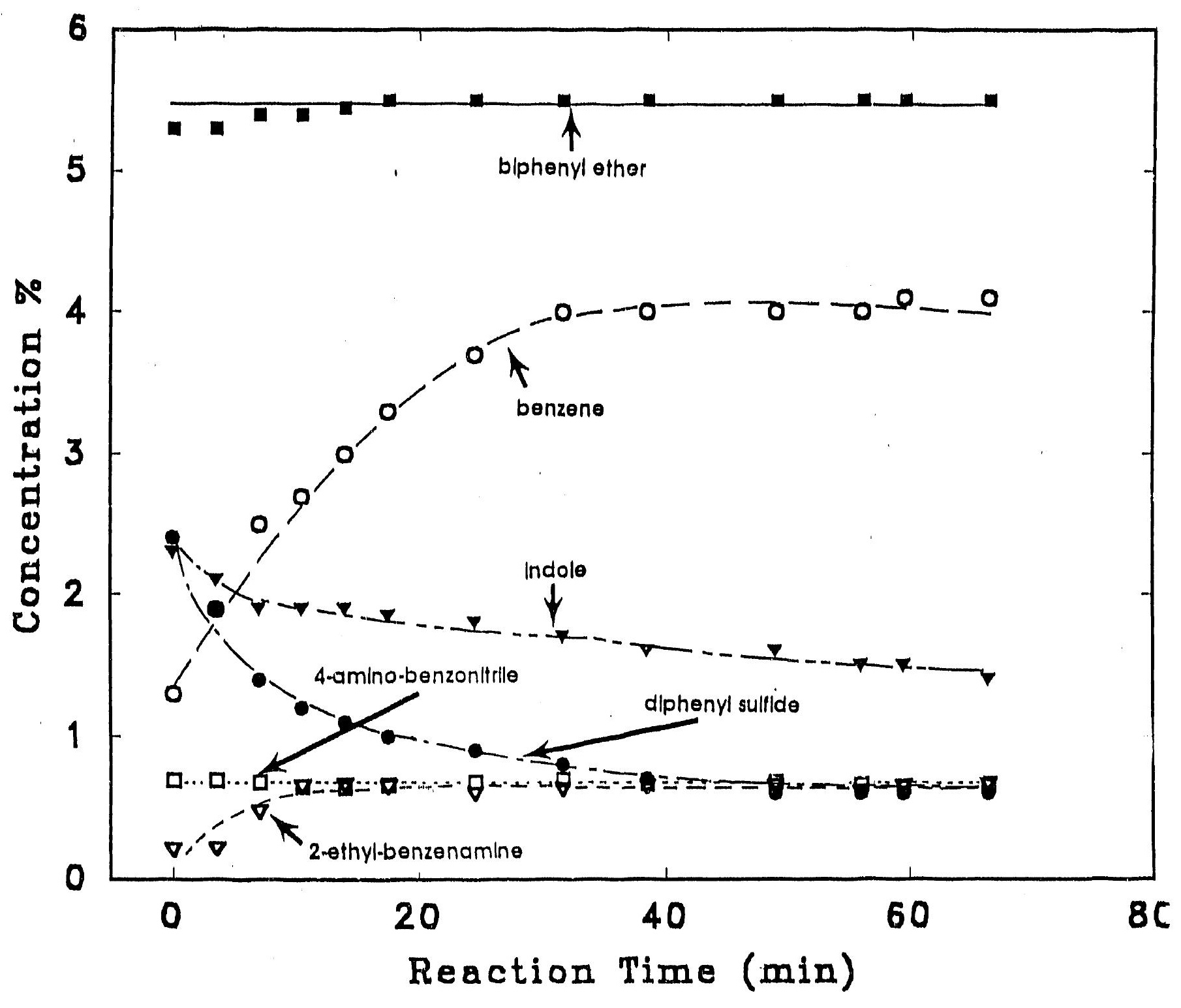

Figure 4. Kinetic profiles of diphenyl ether, indole and diphenyl sulfide conversion $(\square, \nabla, O)$ as well as benzene, 2-ethyl-benzenamine and 4-amino-benzonitrile formrition $(O, \nabla, \square)$ under catalytically controlled conditions. 


\title{
Project II.3 Catalytic Cracking/Hydrogenation and Catalyst Characterization using X-ray Diffraction and SQUID Magnetometry
}

\author{
Mohindar S. Seehra and Manjula M. Ibrahim \\ Department of Physics \\ West Virginia University
}

\section{OBJECTIVES}

During this period, our objectives were to bring to conclusion the characterization of $\mathrm{Fe}_{2} \mathrm{O}_{3} / \mathrm{SO}_{4}{ }^{2 \cdot}$ catalyst using the techniques of $\mathrm{x}$-ray diffraction and magnetometry, with particular attention to determining the particular size distribution. Some efforts were also devoted to the high pressure/high temperature ESR studies of coal hydrogenation.

\section{ACCOMPLISHMENTS/RESULTS}

Detailed experiments and analysis of results have been carried out on $\mathrm{Fe}_{2} \mathrm{O}_{3} / \mathrm{SO}_{4}{ }^{2}$. catalyst, prepared and used by Wender et al [1] in coal liquefaction experiments and on which Huffman et al [2] have reported Mössbauer spectroscopic studies. Our results have been communicated in a recent paper [3], brief summary of which follows. Using a SQUID magnetometer (Quantum Design Model MPMS), magnetization vs. temperature and magnetization vs. field data were taken. After determining the region of superparamagnetism, the distribution function $f(r)$ is determined assuming a log normal distribution and Langevin paramagnetism of superparamagnetic particles. The distribution is found to be fairly symmetric with center near $65 \AA$ and range of 35 to $115 \AA$ for the particle radius (Fig. 1). This analysis assumes spherical particles.

The particle radius has also been determined using the Scherrer equation for line-broadening of Bragg peaks in x-ray diffraction. In Fig. 2(a) and 2(b) we show x-ray diffractograms of the catalyst before and after liquefaction experiments. Before liquefaction, all the major $x$-ray lines of the catalyst can be identified with $\alpha-\mathrm{Fe}_{2} \mathrm{O}_{3}$ whereas after liquefaction, most of the lines correspond to pyrrhotite showing that $\mathrm{Fe}_{2} \mathrm{O}_{3}$ is converted to 
pyrrhotite during liquefaction. To determine the particle size, one needs to subtract the instrumental width $b$ from the experimental width $B$ using $\beta^{2}=B^{2}-b^{2}$, yielding the width $\beta$ due to particle size broadening. Then using the Scherrer equation, $r=0.9 \lambda / 2 \beta \cos \theta(\theta$ is the Bragg angle and $\lambda$ is the $x$-ray wavelength), $r$ can be determined. In our experiments, the instrumental width $b$ was measured for coarse particles of $\alpha-\mathrm{Fe}_{2} \mathrm{O}_{3}$, obtained by powdering a single crystal. Alternately silica can be used as a standard to determine $b$ for different $\theta$. In our case, both methods yielded essentially similar results. In Table 1(a) and 1(b), we show the particle sizes determined by this method. Before liquefaction, the catalyst particle size ranged between $75 \AA$ to $110 \AA( \pm 10 \AA)$ as determined from different Bragg peaks, suggesting that particles are not spherical but that they have anisotropic shape. After liquefaction, the particle size is considerably increased, and as noted earlier, the particles have changed to pyrrhotites (Fig. 2).

For the same catalysts, the Mössbauer spectroscopy measurements of Huffman et al yielded the following information [2]: $12 \%$ of the particles have $<29 \AA$ diameter, $45 \%$ of the particles have diameters from $29 \AA-85 \AA$ and the remaining $43 \%$ of the pa ticles have diameter $>85 \AA$. Thus compared to magnetic studies, Mössbauer spectroscopy yields somewhat smaller sizes whereas $\mathrm{x}$-ray diffraction yields somewhat larger particle sizes. However with the current uncertainty of $\pm 10 \AA$ of these techniques, the results obtained from the three techniques are in good agreement. Magnetic studies have the advantage that a particle size distribution is obtained, with the limitation of the assumption of spherical particles. On the other hand, x-ray diffraction can yield useful information about shape anisotropy, with the added advantage that this technique works for all particles in the range $10 \AA-500$ A. However a particle size distribution is not easily obtainable with $\mathrm{x}$-ray diffraction.

In addition to the above studies, we have also characterized by $x$-ray diffraction several samples of iron carbides prepared by Eklund et al at the University of Kentucky. Details of these results will be reported by this group. Some experiments on the high pressure ESR were also carried out. In the near future, we will be completing experiments on several other catalysts obtained through Huffman et al.

Presentations during this period included the presentations at the Annual CFFLS meeting, 


\title{
Project II.3 Catalytic Cracking/Hydrogenation and Catalyst Characterization using X-ray Diffraction and SQUID Magnetometry
}

\author{
Mohindar S. Seehra and Manjula M. Ibrahim \\ Department of Physics \\ West Virginia University
}

\section{OBJECTIVES}

During this period, our objectives were to bring to conclusion the characterization of $\mathrm{Fe}_{2} \mathrm{O}_{3} / \mathrm{SO}_{4}^{2-}$ catalyst using the techniques of $\mathrm{x}$-ray diffraction and magnetometry, with particular attention to determining the particular size distribution. Some efforts were also devoted to the high pressure/high temperature ESR studies of coal hydrogenation.

\section{ACCOMPLISHMENTS/RESULTS}

Detailed experiments and analysis of results have been carried out on $\mathrm{Fe}_{2} \mathrm{O}_{3} / \mathrm{SO}_{4}{ }^{2-}$ catalyst, prepared and used by Wender et al [1] in coal liquefaction experiments and on which Huffman et al [2] have reported Mössbauer spectroscopic studies. Our results have been communicated in a recent paper [3], brief summary of which follows. Using a SQUID magnetometer (Quantum Design Model MPMS), magnetization vs. temperature and magnetization vs. field data were taken. After determining the region of superparamagnetism, the distribution function $\mathrm{f}(\mathrm{r})$ is determined assuming a log normal distribution and Langevin paramagnetism of superparamagnetic particles. The distribution is found to be fairly symmetric with center near $65 \AA$ and range of 35 to $115 \AA$ for the particle radius (Fig. 1). This analysis assumes spherical particles.

The particle radius has also been determined using the Scherrer equation for line-broadening of Bragg peaks in x-ray diffraction. In Fig. 2(a) and 2(b) we show x-ray diffractograms of the catalyst before and after liquefaction experiments. Before liquefaction, all the major $x$-ray lines of the catalyst can be identified with $\alpha-\mathrm{Fe}_{2} \mathrm{O}_{3}$ whereas after liquefaction, most of the lines correspond to pyrrhotite showing that $\mathrm{Fe}_{2} \mathrm{O}_{3}$ is converted to 
an invited presentation at the Liquefaction Contractors' review meeting in Pittsburgh [4], and a talk at the Pittsburgh Coal Conference in collaboration with Huffman et al.

1. V. R. Pradhan, J. W. Tierney, I. Wender and G. P. Huffman, Energy \& Fuels $\underline{5}, 497$ (1991).

2. G. P. Huffman, B. Ganguly, M. Taghiei, F. E. Huggins and N. Shah, ACS Division of Fuel Chem Reprints 36, 561 (1991).

3. M. M. Ibrahim, J. Zhao and M. S. Seehra, Phys. Rev. B (submitted).

4. M. M. Ibrahim and M. S. Seehra, "Particle Size Distribution of Catalysts. .." 


\section{Attachments}

Table 1. Particle radius $\mathrm{r}$ of the $\mathrm{Fe}_{2} \mathrm{O}_{3} / \mathrm{SO}_{4}{ }^{2-}$, before and after liquefaction, as determined by $x$-ray diffraction.

Fig. 1. Particle size distribution of $\mathrm{Fe}_{2} \mathrm{O}_{3} / \mathrm{SO}_{4}{ }^{2-}$ catalyst determined by magnetometry at 220 $\mathrm{K}$ and $300 \mathrm{~K}$ (from Ref. 3)

Fig. 2. X-ray diffractograms of coarse $\mathrm{Fe}_{2} \mathrm{O}_{3}$ particles, $\mathrm{Fe}_{2} \mathrm{O}_{3} / \mathrm{SO}_{42-}$ catalyst before liquefaction and after liquefaction. 
TABLE I (a)

$\mathrm{Fe}_{2} \mathrm{O}_{3} / \mathrm{SO}_{4}^{2-}$ before liquefaction

\begin{tabular}{l|c|c|c|c|c}
\hline $2 \theta$ & $(\mathrm{hk} \ell)$ & \multicolumn{2}{|c|}{ Spectral widlh } & $\hat{\mu}=\sqrt{\mathrm{B}^{2}-\mathrm{b}^{2}}$ & $\mathrm{r}=\frac{\lambda(0.9)}{2 \beta \cos \theta}$ \\
\cline { 3 - 5 }$\left({ }^{\circ}\right)$ & $\begin{array}{c}\text { B (Sample I }) \\
(10-3 \mathrm{rad})\end{array}$ & $\begin{array}{c}\mathrm{b}(\text { Sample II }) \\
\left(10^{-3} \mathrm{rad}\right)\end{array}$ & $\left(10^{-3}\right.$ iad $)$ & $(\AA)$ \\
\hline 24.18 & $(012)$ & 9.773 & 2.792 & 9.37 & $76 \pm 10^{*}$ \\
33.1 & $(104)$ & 9.599 & 3.491 & 8.94 & $81 \pm 4$ \\
35.54 & $(110)$ & 7.679 & 3.491 & 6.84 & $107 \pm 4$ \\
40.72 & $(113)$ & 7.854 & 4.189 & 6.64 & $110 \pm 10$ \\
49.37 & $(024)$ & 9.076 & 4.625 & 7.81 & $100 \pm 5$ \\
53.98 & $(116)$ & 10.01 & 4.625 & 8.88 & $89 \pm 5$ \\
62.45 & $(214)$ & 10.01 & 4.660 & 8.86 & $93 \pm 10$ \\
63.95 & $(300)$ & 10.01 & 4.018 & 9.17 & $91 \pm 10$ \\
\hline
\end{tabular}

*The quoted uncertainties are due to errors in measuririg $\beta$.

TABLE I (b)

$\mathrm{Fe}_{2} \mathrm{O}_{3} / \mathrm{SO}_{4}^{2-}$ after liquefation

\begin{tabular}{l|c|c|c|c}
\hline $\begin{array}{l}2 \theta \\
\left({ }^{\circ}\right)\end{array}$ & $\begin{array}{c}\mathrm{B} \\
\left(10^{-3} \mathrm{rad}\right)\end{array}$ & $\begin{array}{c}\mathrm{b} \\
\left(10^{-3} \mathrm{rad}\right)\end{array}$ & $\begin{array}{c}\beta^{\beta} \\
\left(10^{-3} \mathrm{rad}\right)\end{array}$ & $\mathrm{r}(\AA)$ \\
\hline 24.92 & 5.916 & 2.792 & 5.216 & 135 \\
33.82 & 5.742 & $3.49 !$ & 4.560 & 159 \\
26.675 & 4.785 & 3.314 & 3.451 & 206 \\
43.75 & 7.656 & 4.011 & 6.521 & 114 \\
53.14 & 6.960 & 4.360 & 5.25 & 142 \\
\hline
\end{tabular}




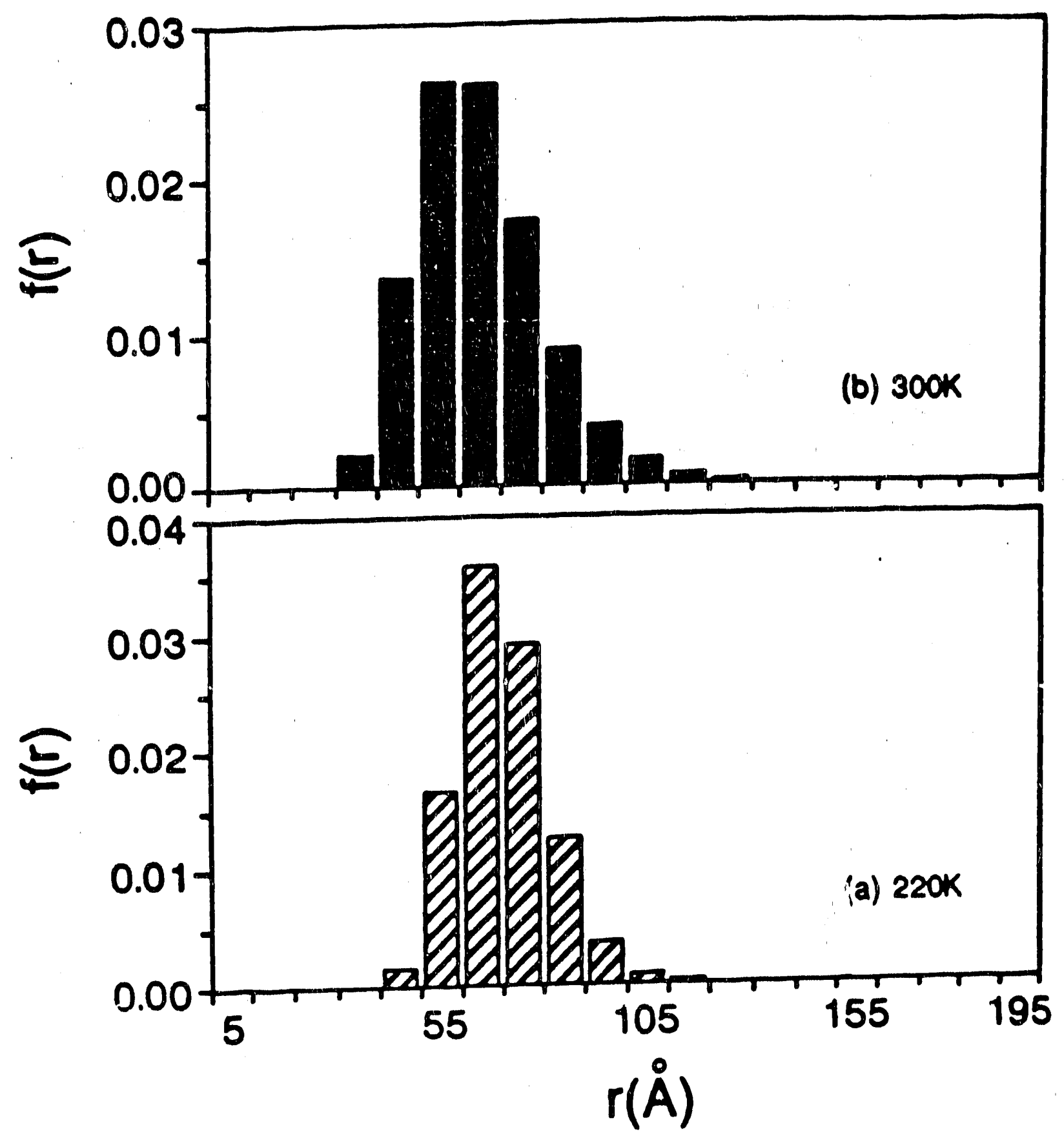

Fig. 1. Particle size distribution of $\mathrm{Fe}_{2} \mathrm{O}_{3} / \mathrm{SO}_{4}^{2-}$ catuyst determined by magnetometry at $220 \mathrm{~K}$ and $300 \mathrm{~K}$ (from Ref. 3) 


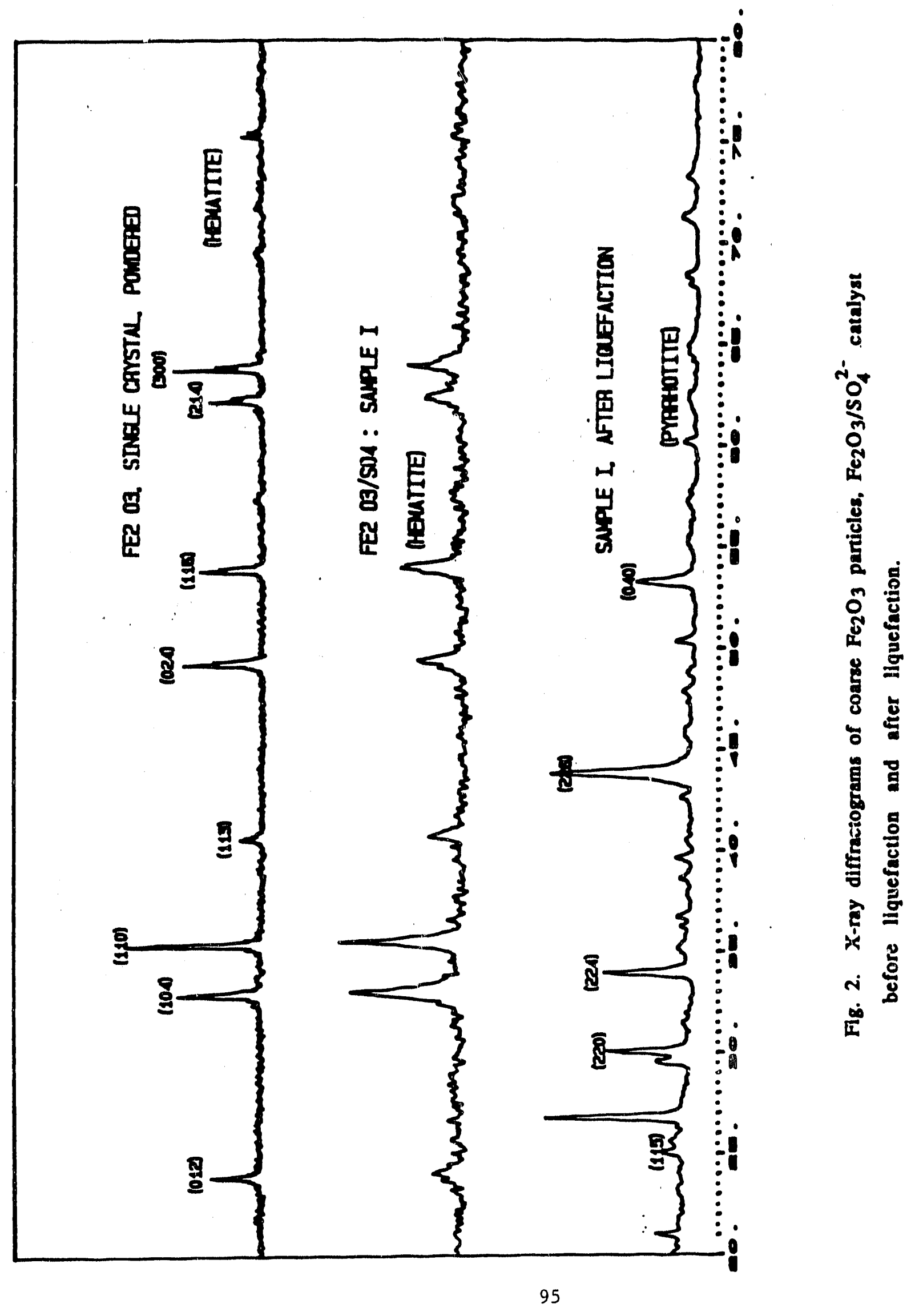




\title{
Project II.4 ESR and ENDOR Investigations for Coal Liquefaction Under Mild Conditions
}

\author{
Naresh Dalal and Jeffrey P. DeLooze \\ Department of Chemistry \\ West Virginia University
}

\section{INTRODUCTION}

In our previous (1 May - 31 July 1991) CFFLS presentation', we discussed our work on the ENDOR methodology developed in the investigation of the formation of free radicals in the superacid-catalyzed electron transfer reactions of a variety of organic compounds. The compounds selected were ring-substituted, resonance-stabilized, aromatic substances that are generally considered as good models for the organic moieties. These results have added to the knowledge base already established in our preliminary ESR investigations of these compounds during the first quarter ${ }^{2}$ of the current year. As per schedule, for this quarter our expected goals were the following:

1. To utilize ESR spectroscopy to characterize the relaxation behavior of coal chars and residues to determine the experimental conditions under which ENDOR spectroscopy can be performed. This is necessary as our long-range goal " the program is to extend our ENDOR techniques beyond the $200^{\circ} \mathrm{C}$ limit currently attainable. In doing so, we hope to extend the methodoiogy to ascertain the ionic character of heterogeneously catalyzed simple model comprands proposed for use in further Consortium work. The charge information will be obtained via measurements of proton hyperfine splittings. For example, for a given molecule, the cations typically exhibit $\approx 20 \%$ larger spin densities as compared to the anions, with the neutral species exhibiting an average behavior. Such differences are easily measurable via ENDOR

\footnotetext{
1 Dalal, N.S., and Delooze, J.P., Fifth Annual Technical Meeting of the Consortium for Fossil Fuel Liquefaction science, Lexington, KY, August 13, 1991.

2 Dalal, N. S. and J. P. Delooze, CFFLS First Quarterly Report, 1991.
} 
spectroscopy ${ }^{3}$.

2. Provide a check for the correlation between NMR relaxation measurements as provided by Ron Pugmire's group with our own measurements as determined by line saturation behavior. This allows us to cross-validate our technique against an established methodology for the determination of ENDOR feasibility.

The technique utilized was the lineshape saturation technique for the determination of the relaxation times for the electron spin state in Utah coal chars provided by Dr. Ron Pugmire of the University of Utah. In this technique, the compound is subjected to different microwave power levels while the area of the ESR transition peak is monitored. Under saturation conditions, an increase in power causes either no increase or a decrease in signal intensity. This behavior is described by the modified Bloch equations ${ }^{4}$ which provide that:

$$
\begin{gathered}
Y^{2} H_{1}^{2} T_{1} T_{2} \approx 0.5 * S^{-1} \\
T_{2}=\frac{2}{\sqrt{3} Y \Delta H_{p p}^{0}}=\frac{1.3131 \times 10^{-7}}{g \Delta H_{D p}^{0}} \\
T_{1}=\frac{\sqrt{3} \Delta H_{D p}^{0}}{2 Y}\left(\frac{S^{-1}-1}{0.25 H_{1}^{2}}\right)
\end{gathered}
$$

\section{EXPERIMENTAL}

All ESR measurements were made at the X-band $(9.5 \mathrm{GHz})$ frequencies using a Bruker ESR/ENDOR spectrometer, model ER-200D/ESP-3220. The sample holder was specially designed to enable in-situ evacuation to $10^{-4}$ torr, or to pressurize with helium or any other gas at a known pressure and temperature. All data collection and experimental calculation were carried out using a Bruker ESP-3220 microcomputer and laboratory-developed

\footnotetext{
3 Dalal, N.S., and DeLooze, J.P., paper presented at the Fourth Annual CFFLS Technical Meeting, Snowbird, Utah (1990).

4 Wertz, J. E. and J. R. Bolton, Electron Spln Regonance: Elementary Theory and Practical Applications, New York: Chapman and Hall, pp. 196-201 (1986).
} 
ESR/ENDOR data collection and simulation programs.

\section{RESULTS AND DISCUSSION}

Figure 1 shows the ESR saturation behavior of two Utah chars, one heated at $200^{\circ} \mathrm{C}$ and one heated at $500^{\circ} \mathrm{C}$. As is seen in Figure 1, the lower temperature char shows the saturation effect while the high temperature char does not. This implies that the $200^{\circ} \mathrm{C}$ char is a more suitable candidate for the ENDOR technique. It implies that this ESR transition, which will be probed via the ENDOR technique, can establish a suitable spin population difference for the detection of strong ENDOR response. It does not imply, however, that the $500^{\circ} \mathrm{C}$ char is incapable of saturation - only that at room temperature, the $200^{\circ} \mathrm{C}$ char is a more suitable ENDOR candidate than the $500^{\circ} \mathrm{C}$ char. Solution of the modified Bloch equations provide $T_{1}$ values of $\approx 10 \mathrm{~ms}$ for the $200^{\circ} \mathrm{C}$ char and $\leq 1 \mu \mathrm{s}$ for the $500^{\circ} \mathrm{C}$ char, in reasonable agreement with the data from the Utah NMR group.

Figure 1: Saturation Effect in example Utah Chars 

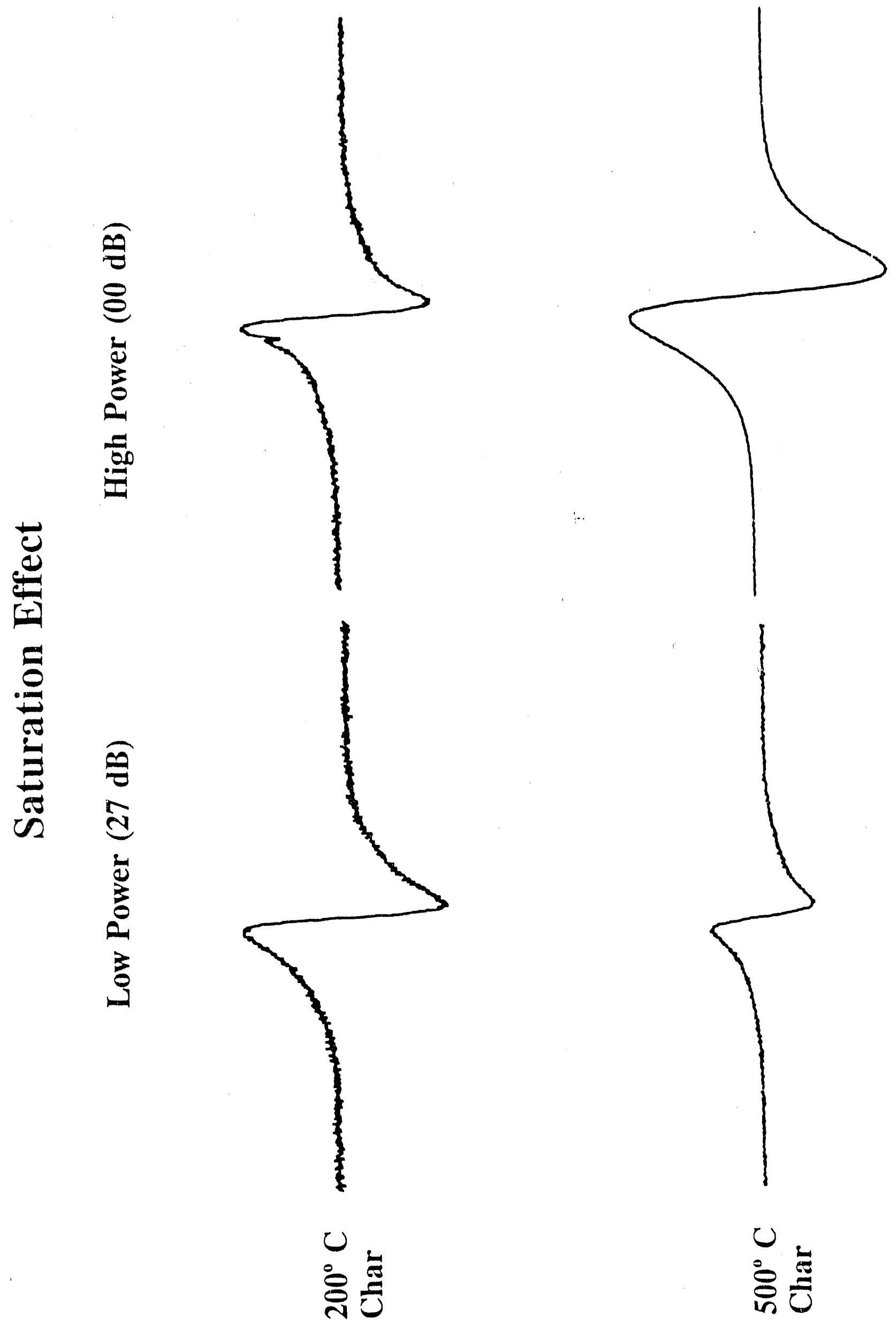

蒿衰 
Figure 2 shows the variation in iinear scale peak area versus the $\log _{10}$ scale microwave power. As is clearly seen, the integrated area for the $500^{\circ}$ and $600^{\circ}$ chars are increasing with respect to the $\log _{10}$ scale microwave power. However, the $200^{\circ} \mathrm{C}$ char appears to possess a flat plot of integrated area with respect to the $\log _{10}$ scale microwave power: this implies that the $200^{\circ} \mathrm{C}$ char is easily saturated and ENDOR is easily performed. Chars examined were: ambient, $200^{\circ} \mathrm{C}, 300^{\circ} \mathrm{C}, 400^{\circ} \mathrm{C}, 500^{\circ} \mathrm{C}, 600^{\circ} \mathrm{C}$ and $900^{\circ} \mathrm{C}$, with saturation behaviors linearly consistent with heating (i.e., $200^{\circ} \mathrm{C}$ char most saturable and $900^{\circ} \mathrm{C}$ char least saturable.)

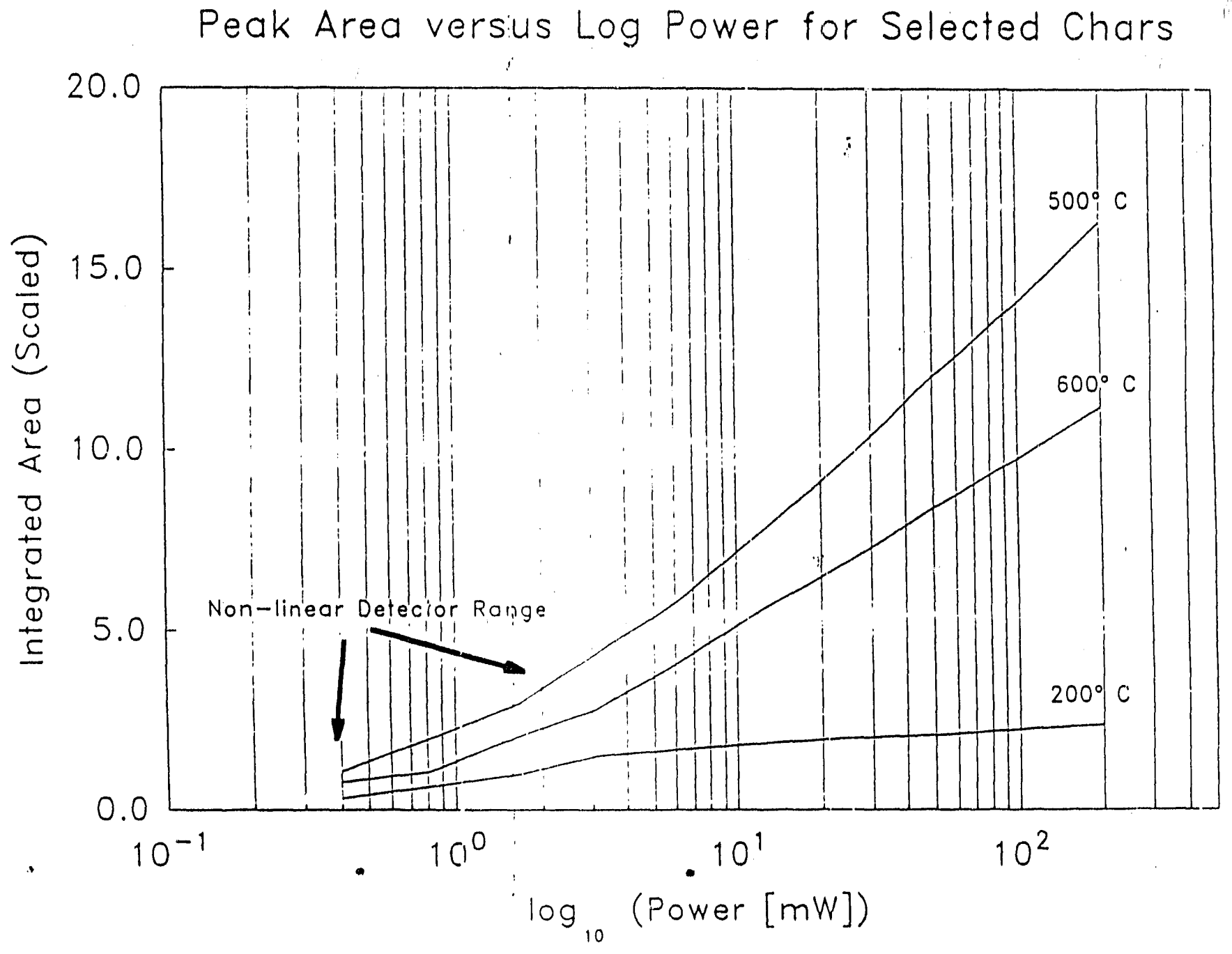

Figure 2: Peak Area versus Microwave Power (Log Scale) for Selected Chars (others omitted for clarity). 


\title{
Project П.5 Depth Profile Studies on Catalyst and Catalyst Impregnated Coal Samples
}

\author{
P.J. Reucroft and J.Y. Kim \\ University of Kentucky
}

In a previous report, attempts to characterize the surface of six catalysts and catalyst : $\left(\mathrm{FeOOH} / \mathrm{SO}_{4}{ }^{2-}\right)$ impregnated Illinois \#6 coa: wore described. This preliminary characterization showed that the concentration of elements at the outermost layers measured by XPS was different from that in the bulk determined by chemical analysis. The oxygen concentration was enriched in the surface regions of all the samples. Results on the oxidation states of $\mathrm{Fe}, \mathrm{Mo}$ and $\mathrm{Sn}$ showed that the most stable oxidation states of these elements were generally observed.

In the present work, depth profiling studies have been carried out to determine if there are any differences in the distribution of the major components in catalyst II $\left(\mathrm{Mo} / \mathrm{FeOOH} / \mathrm{SO}_{4}{ }^{2}\right)$ and catalyst $\mathrm{I}\left(\mathrm{FeOOH} / \mathrm{SO}_{4}{ }_{4}{ }^{2}\right)$ impregnated Illinois $\# 6$ coal samples. The samples have been analyzed by is combination of Ar sputtering with XPS measurements. Due to the edge effect associated with the unetched sample surface, the depth profiles for materials in powder form were difficult to obtain and the elemental distribution changed with increasing sputtering times. Preferred areal sputtering was carried out to reduce this effect. Although different features such as preferential sputtering, atomic mixing, particle size effects, etc., can effect the etching profiles, sputtering was performed in the present studies for comparative purposes on samples where such effects were expected to be very similar. All XPS measurements employed the same conditions that were described previously. ${ }^{1}$ Art sputtering of the powdered samples was carried out with the Art gun operating at $3.5 \mathrm{kV}$ (estimated etching rate of ca. $20 \mathrm{~A} / \mathrm{min}$, as determined from a $\mathrm{SiO}_{2}$ standard film). No charge compensation with the electron flood gun was utilized in these initial etching experiments.

Figures 1 and 2 show depth profile concentration profiles for the most abundant elements in catalyst I and catalyst I impregnated Illinois $\$ 6$ coal samples, respectively. A systematic trend towards lower weight concentrations of both oxygen and sulfur was 
observed for both samples as sputtering time increases (i.e. when going from surface to the bulk). A relatively high oxygen concentration was determined initially at the surface by XPS before etching, but a sharp drop in oxygen concentration was subsequently observed as shown in Fig. 3. This surface enrichment of oxygen can be ascribed to air oxidation and the oxidized layer thickness was estimated to be 40-50 A. In Figure 1, iron shows an enrichment as the etching proceeds from the surface to the bulk of catalyst $I$. This was also observed in Fig. 4 for catalyst II. From these results, which show a decrease in oxygen and an increase in iron concentrations with increased etching, it can be concluded that the surface iron particles are covered with a thin oxygen layer. However, no change was observed in the iron concentration in the case of the catalyst I impregnated coal sample shown in Figure 2. If can thus be assumed that the iron particles in these samples are encapsulated by organic materials from the coal.

Chemical state changes for sulfur were observed as the etching proceeded in both samples. The surface sulfur showed a binding energy of $168.8 \mathrm{eV}$, indicating sulfate $\left(\mathrm{SO}_{4}{ }^{2-}\right)$ and no peaks were observed in the sulfide region $(164 \mathrm{eV})$ in the case of the catalyst I sample. As sputtering time increased, however, the sulfate peak decreased and finally disappeared. A sulfide or elemental sulfur peak was then observed. These results are shown $\mathrm{n}$ Figs. 5 and 6 . This can be ascribed to the removal of oxidized sulfur at the surface by the etching procedure. No chemical state changes were observed for Fe and Mo states

as a result of etching. Further analysis of the effect of etching on the sulfur peak is in progress. Figure 7 compares the wide scan spectra for the catalyst I impregnated coal sample before and after Ar+ sputtering.

\section{REFERENCE}

1. P.J. Reucroft and J.Y. Kim, Annual Report for CFFLS (1990 - 1991) 
Weight $x$

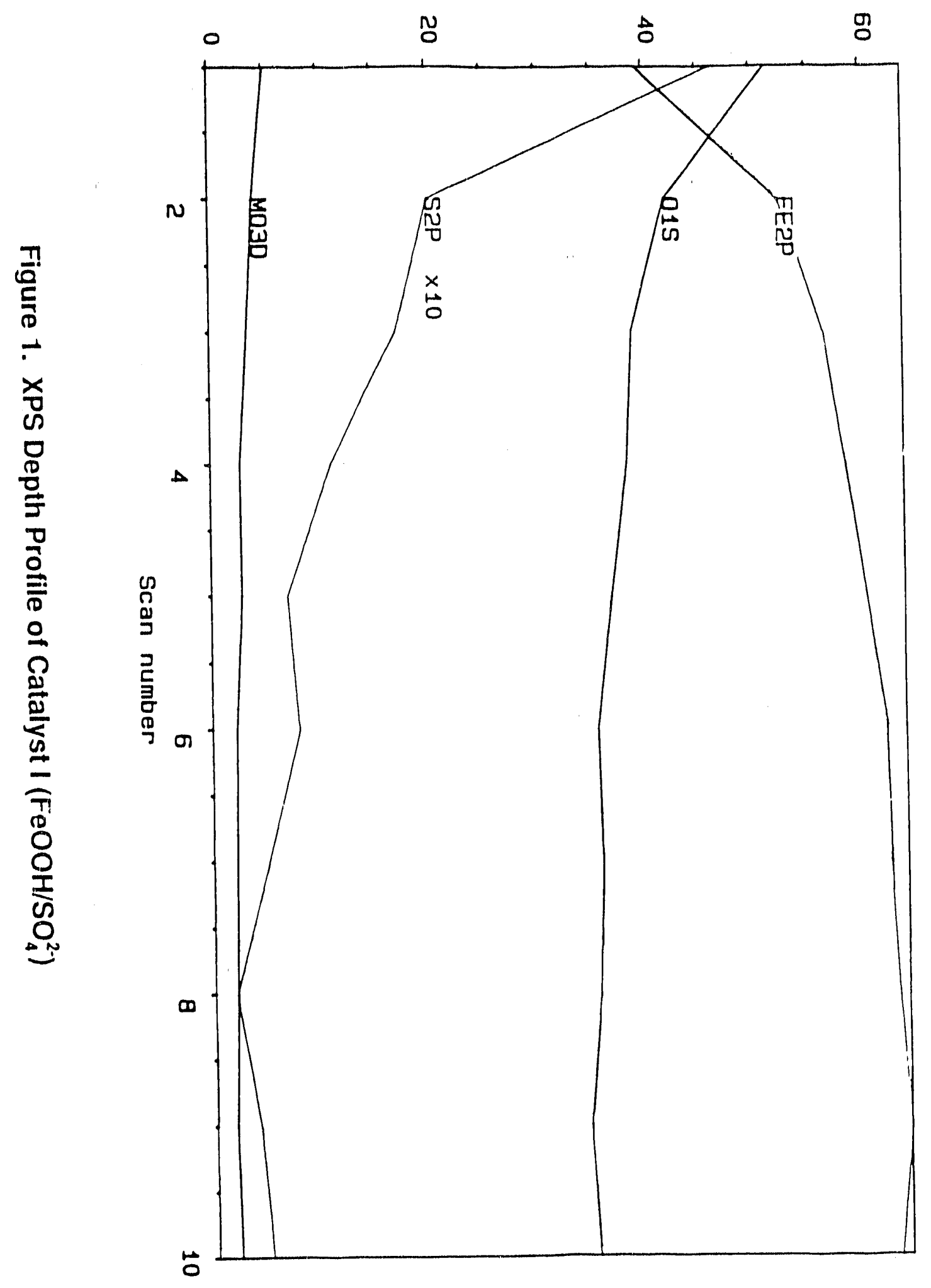




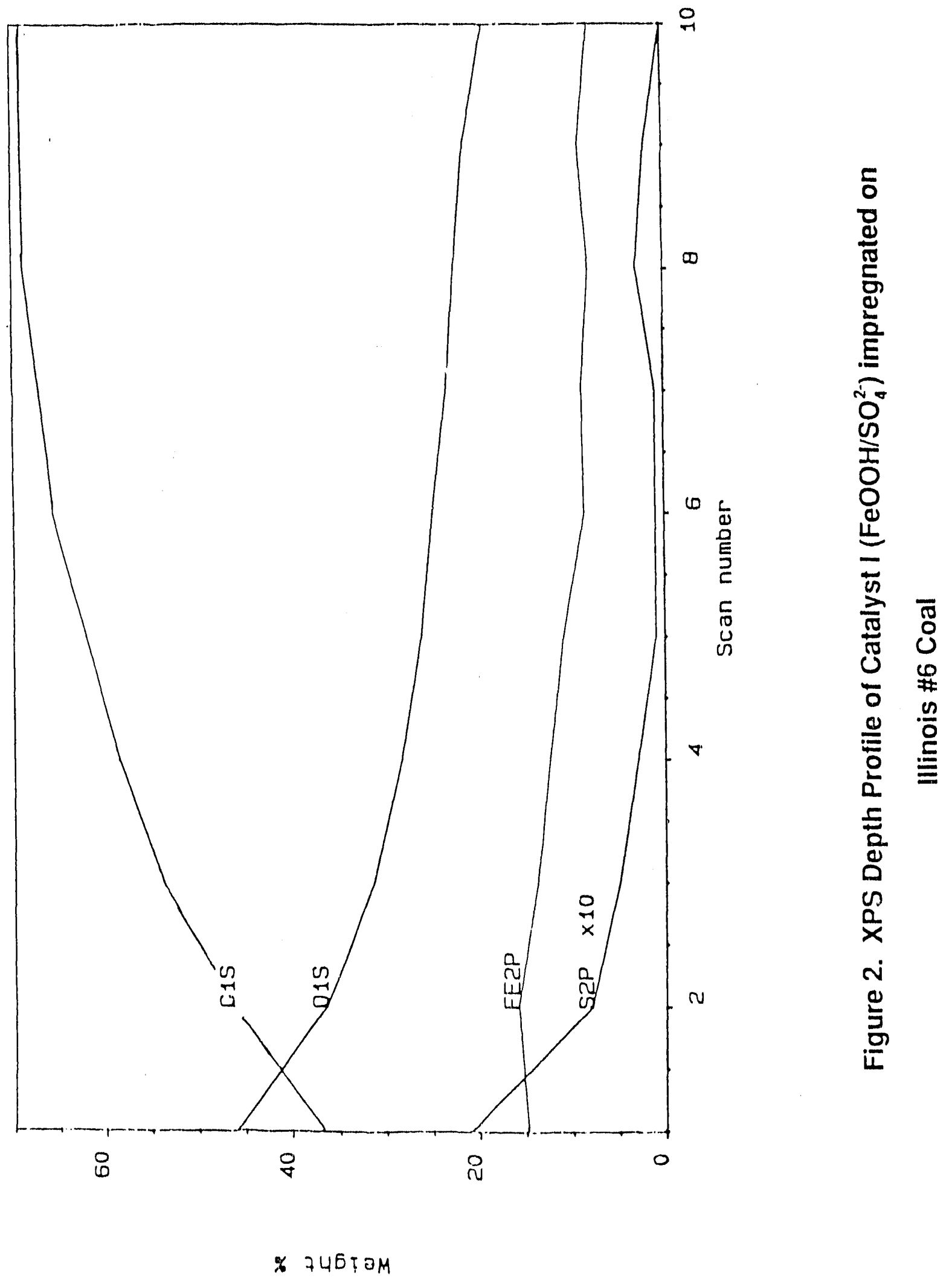



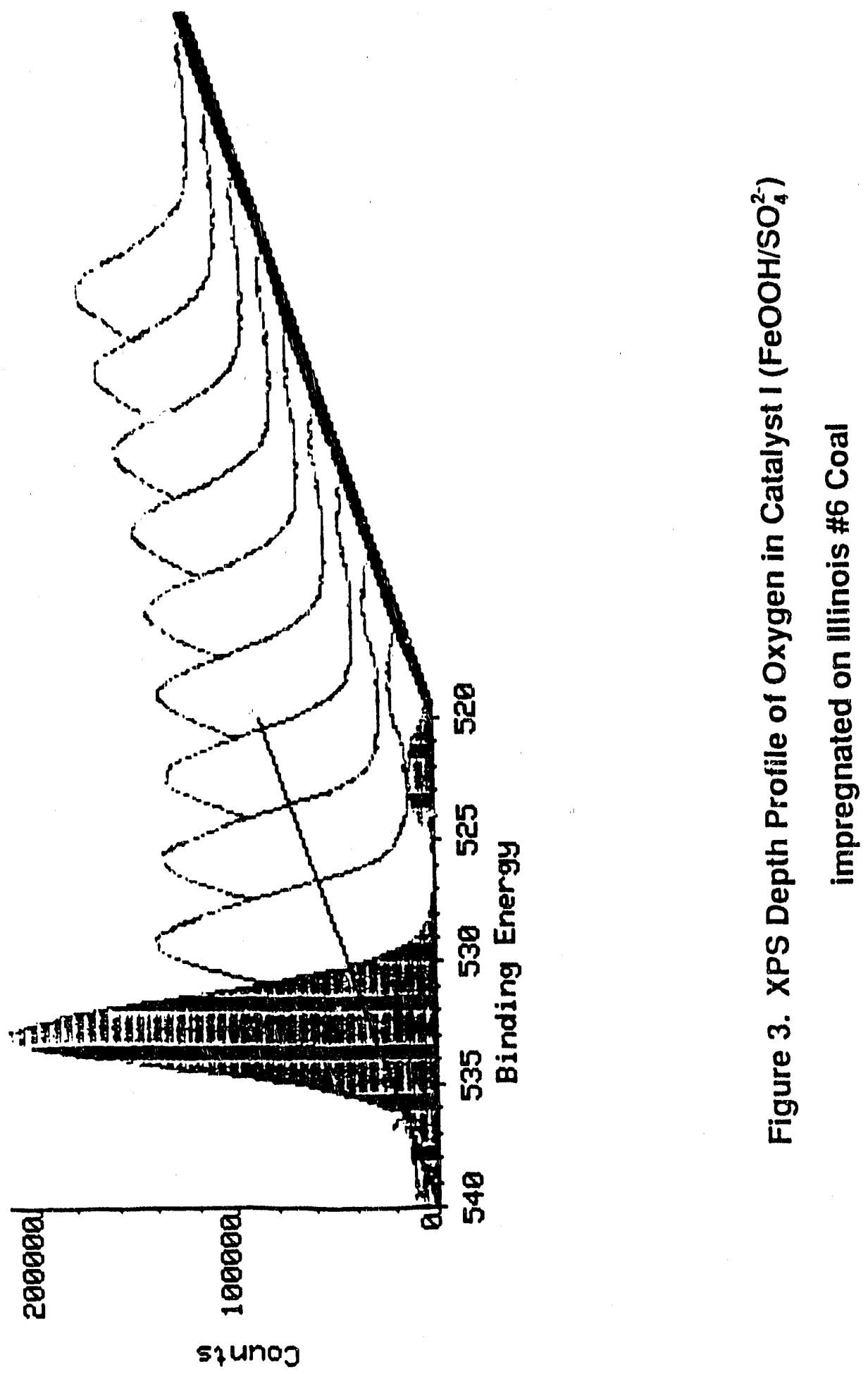


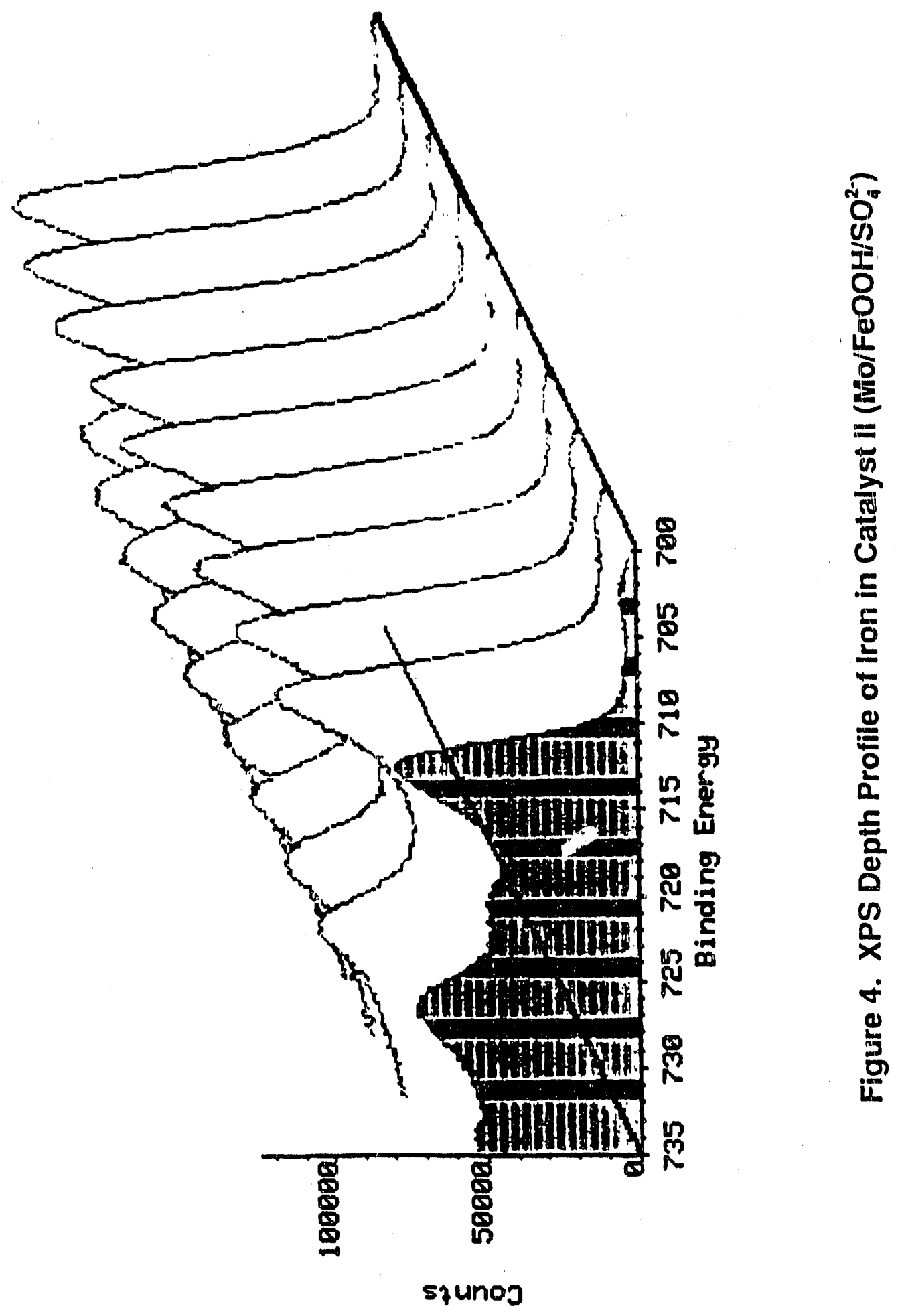




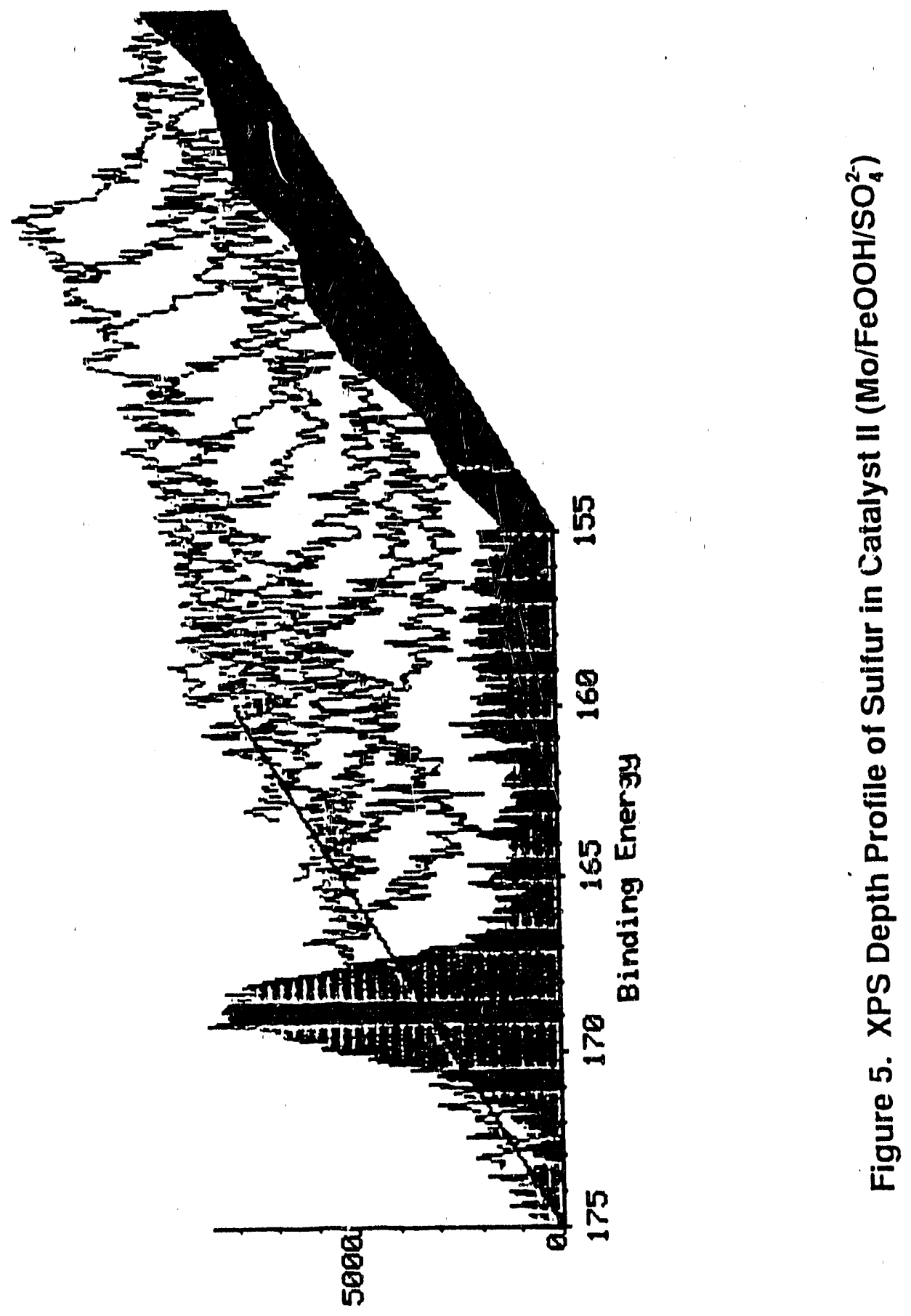

squno? 

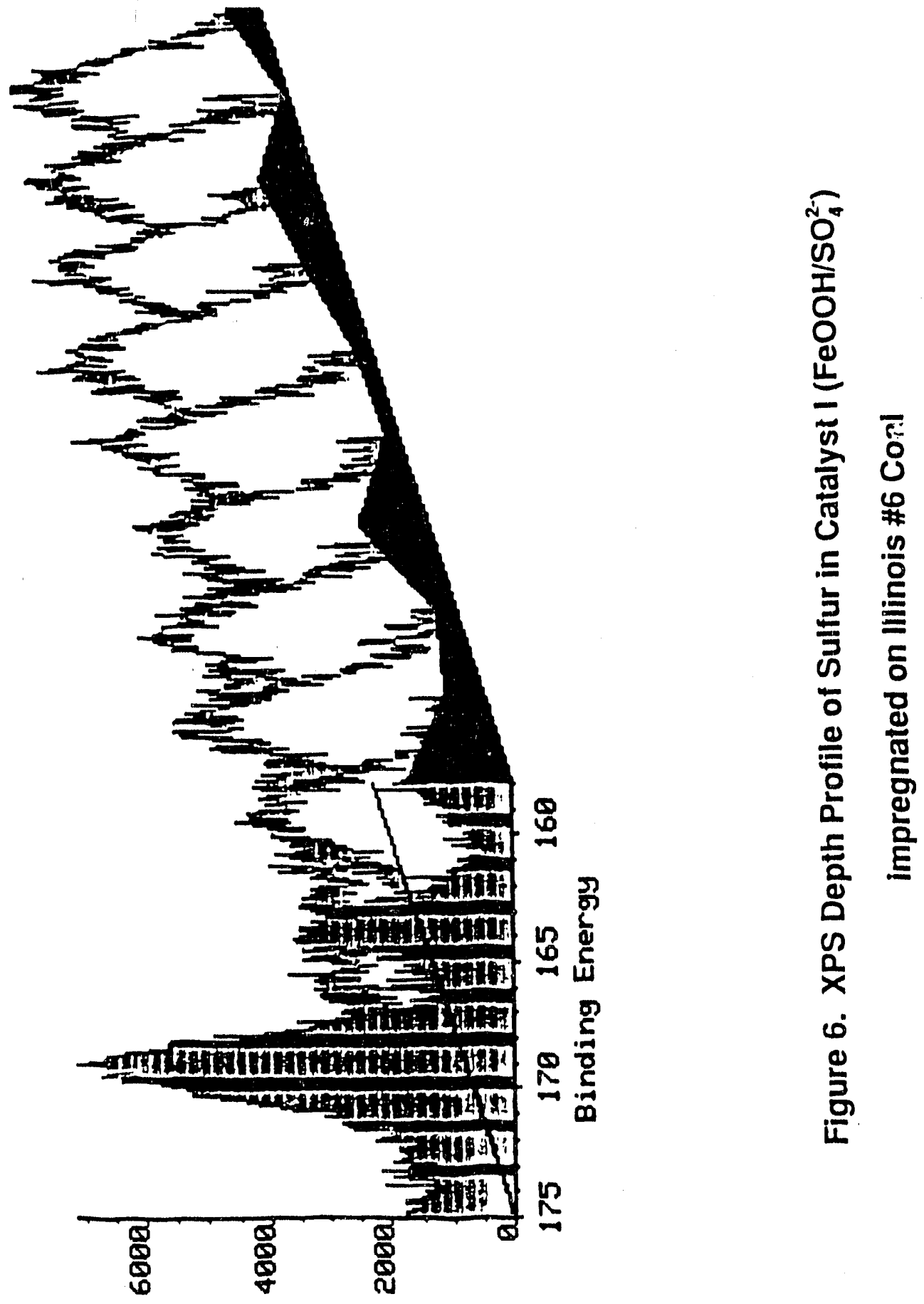

squnoj 


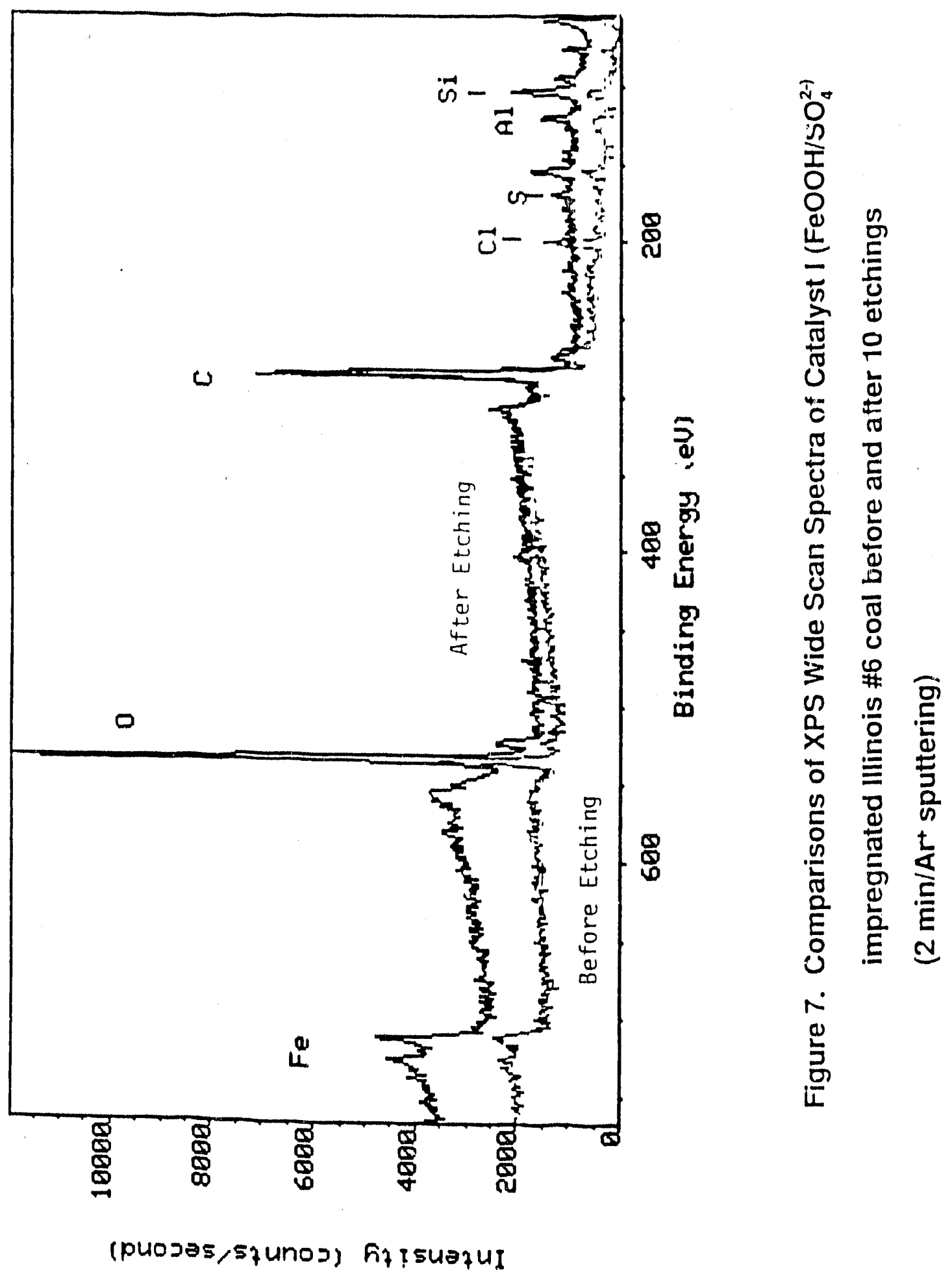




\title{
Project II.6 Computational Chemistry of Model Compounds and Molecular Fragments of Relevance to Coal Liquefaction
}

\author{
K.R. Subbaswamy \\ University of Kentucky
}

Progress was made on three fronts:

1. Continued investigation of radical cation transfer mechanism as an explanation for the catalytic effect of carbon black in the cleavage of the carbon bond linking polycyclic condersed aromatic rings and aliphatic carbon atoms in a series of model compounds that have structural features common in coal fragments. We have received additional experimental data from Dr. Melvina Farcasiu, and are performing calculations.

2. Continued development of molecular dynamics method for including the effect of temperature in microscopic simulation of reactions. As a test case, the method was applied to study the chemisorption of oxygen on the surface of $\mathrm{C}_{60}$. This paper has been submitted to Physical Review Letters.

3. Investigation of the radical hydrogen transfer mechanism as a possible explanation for the observed catalytic effects of selective bond cleavage in model compounds. The following pages give a detailed report of our findings (manuscript in preparation).

Farcasiu and coworkers ${ }^{1-3}$ have recently studied a series of model compounds with structural similarities to coal molecule fragments. They have found that carbon black catalysts show remarkable selectivity for the cleavage of the bond linking a naphthyl ring to an aliphatic carbon. Combining reactivity studies with a charge distribution analysis of the catalyst, Farcasiu and coworkers have proposed that, in the presence of the catalyst, the reaction proceeds via the formation of a radical cation, with the positive charge concentrated on the naphthyl ring. Some semiempirical quantum chemical calculations performed by $\mathrm{us}^{4-7}$ have supported this proposal.

Radical hydrogen transfer ${ }^{8.15}$ (RHT) and protonation (or acid catalysis) are important alternative mechanisms for the cleavage of strong bonds in this context. ${ }^{16}$ In this paper we perform AM1 quantum chemical calculations to test whether the RHT mechanism can account for the experimental results observed by Farcasiu, et al.

The AM1 calculations have been done with the quantum chemical program, MOPAC 5.0 , of Stewart. ${ }^{17}$ The widely used MNDO (modified neglect of diatomic overlap) and AM1 (Austin Model 1) methods developed by Dewar, et al. ${ }^{18,19}$ are based on the neglect of diatomic 
differential overlap (NDDO) approximation. The valence shell molecular orbitals are represented by linear combinations of a minimum basis set of valence set atomic orbitals (Xi):

$$
\phi_{j}=\sum_{i} c_{i j \lambda_{i}}
$$

The wave function for the many-electron system is approximated as a single determinant

$$
\Psi=\left|\phi_{1}(1) \phi_{2}(2) \phi_{3}(3) \cdots\right|
$$

and a Hartree-Fock type calculation is performed within the NDDO semi-empir cal treatment. Since the theoretical bases for such semi-empirical methods may be found in text books, ${ }^{20}$ further details wisl not be given here.

The MNDO and AM1 molecular orbital methods have been heavily parametrized to give good heats of formation and minimum energy geometries for neutral organic molecules. In general, the minimum energy geometries and heats of formation calculated with these methods are very good. ${ }^{18,19}$ AM1 is a version of $\mathrm{MNDO}$ that has been reparameterized so as to describe hydrogen bonding correctly. ${ }^{19}$ Our previous experience with this method has been that while its results are quite reliable for neutral species, its ability to handle charged species with existing parametrization is questionable. Since the RHT mechanism only involves neutral species at all stages, the AM1 results should be reliable.

Our results in this paper are for 4-(1-naphthylmethyl)bibenzyl (Model I) ${ }^{1}$, for which the most extensive experimental results are available. A planar representation of the molecule is given in Fig. 1. Full geometry optumization, except for keeping aromatic rings planar, is performed for the parent as well as the products.

RHT basically involves two steps: the bimolecular transfer of a hydrogen atom from a soivent derived radical to the ipso position of an aryl-alkyl linkage of the substrate followed by the elimination of the substitutent. Free hydrogen atom transfer may also compete with the RHT process. The outcome of the competition is governed by many factors including aromaticity of the hydrogen donor. ${ }^{10}$ The processes involved in RHT followed by cleavage of the relevant bond are controlled by four energies as illustrated in Fig. 2: $E_{1}$ is the activation barrier for the transfer of the hydrogen atom from the solvent radical to the ipso site of the substrate; $E_{2}$ is the energy released in the formation of the hydrogen-substrate radical complex; $E_{3}$ is the activation barrier for the separation of the fragments, and $E_{4}$ is the dissociation energy of C-C bond that is cleaved.

To calculate the various energies in the AM1 framework, a hydrogen atom was allowed to approach Model $I$ in each of the four positions studied, as indicated in Figure 1. Since we are interested in relative energies of hydrogen transfer from the solvent radical to different positions in the same substrate, it was felt that the activation energy for the transfer 
could be calculated, to a first approximation, by allowing a hydrogen atom to approach the molecule in the indicated positions, rather than as a transfer from the solvent derived radical. This makes the calculation much more computationally tractable. Also, the hydrogen donor solvent used by Farcasiu and co-workers in their experimental studies was 9,10dihydrophenanthrene. Malhotra, et al ${ }^{10,12}$ found that, in a phenanthrene-dihydrophenanthrene hydrogen donor solvent system, the cleavage of 1,2'-dinaphthylmethane via free hydrogen atoms was more important than in an anthracene-dihydroanthracene solvent system. The relevant $\mathrm{C}-\mathrm{H}$ distance was fixed and all other geometric coordinates were optimized for each fixed $\mathrm{C}-\mathrm{H}$ distance. After addition of the hydrogen atom to the compound, the $\mathrm{C}-\mathrm{C}$ bond was allowed to elongate, again with all geometric coordinates except for the fixed C-C distance being optimized.

Our AM1 results for the four energies for hydrogen transfer to positions $1,2,3$, and 4 are sliown in Table 1 with the labels given in Figure 1. The barriers for the addition of hydrogen to the compound in the four different positions are all similar, but the addition of the hydrogen to the carbon atom of the naphthyl ring requires slightly more energy than the addition to the phenyl ring carbons. After the addition of hydrogen, the radical in which the hydrogen has added to the naphthyl ring is more stable by at least $0.11 \mathrm{eV}$ than the other possible radical complexes. The activation energy for the cleavage of the $\mathrm{C}-\mathrm{C}$ bond is highest for bond $\mathrm{c}$ and lowest for bond $\mathrm{B}$, while the bond dissociation energy is lowest for bond $\mathrm{e}$. However, there is not a large difference in any of these energies, especially when one takes into consideration an experimental temperature of $400^{\circ} \mathrm{C}$.

It is thought that the second step in RHT, namely the cleavage of the ipso bond of the radical complex, is the fast step. Therefore the barrier height of the first step should control the final product distributions. The distributions that result from the consideration of the relative activation energies of this first step are listed in Table 1. With the kinetic analysis of bond cleavage through a radical hydrogen transfer, we do not see the same marked preference for bond cleavage that is evident in the experimental results (cleavage of the bond adjacent to the naphthyl group, bond a) nor in the results of the ASED-MO calculations of our earlier work and, as such, conclude that radical hydrogen transfer, while a possible mechanism for the cleavage of the strong bonds, does not seem to be the dominant mechanism operation in the catalytic cleavage by carbon black of Model I.

Acknowledgement. The authors would like to thank the University of Kentucky Center for Computational Sciences for partial support for this project. We are grateful to Dr. Malvina Farcasiu for suggesting the study of 4-(1-naphthyl)bibenzyl and for providing experimental data. We would also like to thank Dr. Paul Corio for our many discussions about interpretation of the kinetic analysis. 


\section{REFERENCES}

[1] Farcasiu, M.; Smith, C. Prepr. Pap.-Am. Chem. Soc., Div. Fuel Chem., 1990, 35, 404.

[2] Farcasiu, M.; Smith, C. Energy Fuels, 1991, 5, 83.

[3] Farcasiu, M.; Smith, C.; Hunter, E.A. (to be published).

[4] Ades, H.F.; Companion, A.L.; Subbaswamy, K.R. J. Phys. Chem., 1991, 95, 2226.

[5] Ades, H.F.; Companion, A.L.; Subbaswamy, K.R. Prepr. Pap.-Am. Chem. Soc., Div. Fuel Chem., 1991, 36, 420.

[6] Ades, H.F.; Companion, A.L.; Subbaswamy, K.R. J. Phys. Chem., 1991, 95, 6502.

[7] Ades, H.F.; Companion, A.L.; Subbaswamy, K.R. (to be published).

[8] Autrey, T.; Franz, J.A. Prepr. Pap.-Am. Chem. Soc., Div. Fuel Chem., 1991, 36, 381.

[9] McMillen, D.F.; Malhotra, R.; Tse, D. Prepr. Pap.-Am. Chem. Soc., Div. Fuel Chem., 1991., 36, 498.

[10] Malhotra, R.; McMillen, D.F.; Energy Fuels, 1990, 4, 184.

[11] Autrey, T.; Gleicher, G.J.; Camaioni, D.M.; Ferris, K.F.; Franz, J.A. Prepr. Pap.-Am. Chem. Soc., Div. Fuel Chem., 1991, 36, 521.

[12] McMillen, D.F.; Malhotra, R.; Chang, S-J.; Ogier, W.C.; Nigenda, S.E.; Fleming, R.H. Fuel, 1987, 66, 1611.

[13] Metzger, J.O. Angew. Chem., Int. Ed. Engl., 1986, 25, 80.

[14] McMillen, D.F.; Malhotra, R.; Nigenda, S.E. Fuel, 1989, 68, 380.

[15] Freund, H.; Matturo, M.G.; Olmstead, W.N.; Reynolds, R.P.; Upton, T.H. Energy Fuels, $1991,5,840$.

[16] Poutsma, M.L. Energy Fuels, 1990, 4, 113.

[17] Stewart, J.J.P.; et al. Quantum Chemistry Program Exchange No. 581, vers. 5.0, (Indiana University, Bloomington).

[18] Dewar, M.J.S.; Thiel, W. J. Am. Chem. Soc., 1977, 99, 4899. 
[19] Dewar, M.J.S.; Zoebisch, E.G.; Healy, E.F.; Stewart, J.J.P. J. Am. Chem. Soc., 1985, 107, 3902.

[20] Sadlej, J. Semi-Empirical Methods of Quantum Chemistry John Wiley \& Sons: New York, 1985.

[21] Anderson, A.B. J. Chem. Phys., 1974, 60, 2477. 


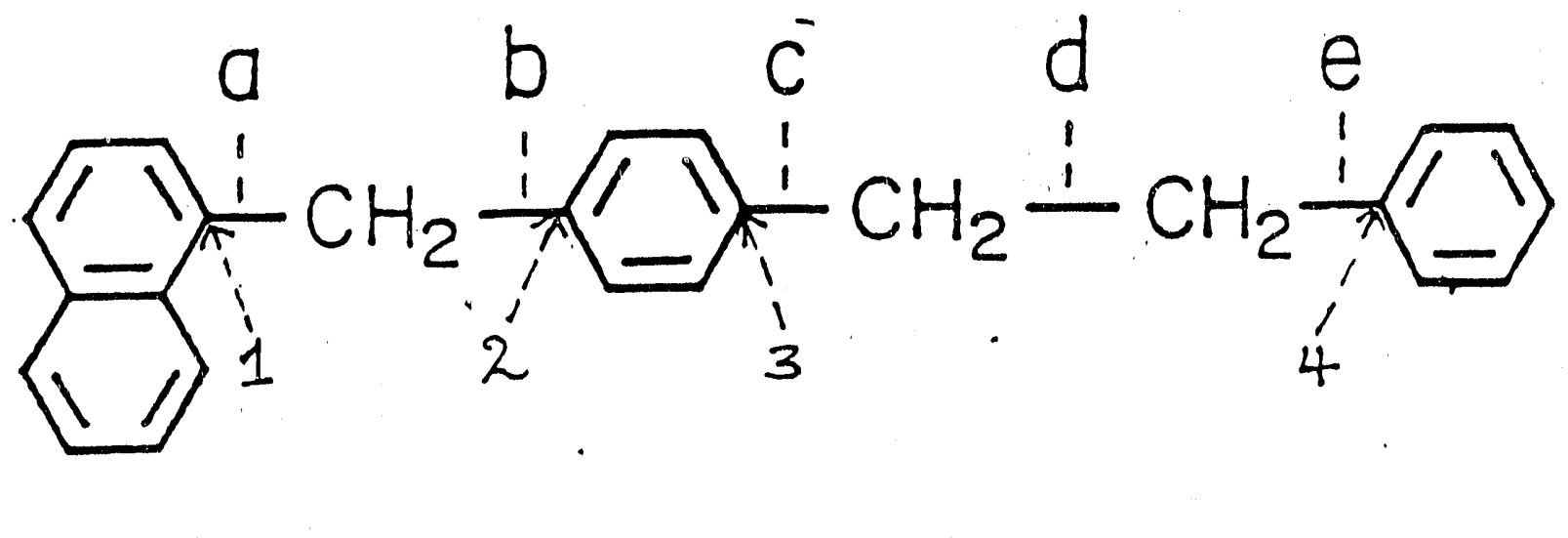

Figure 1: Model I

\begin{tabular}{lccccc}
\hline \hline Position & $E_{1}$ & $E_{2}$ & $E_{3}$ & $E_{4}$ & \%Distribution \\
1 & 0.57 & 1.58 & 1.30 & 0.59 & 7 \\
2 & 0.51 & 1.40 & 1.21 & 0.48 & 19 \\
3 & 0.44 & 1.42 & 1.36 & 0.52 & 64 \\
4 & 0.55 & 1.35 & 1.29 & 0.51 & 10 \\
\hline \hline
\end{tabular}

Table 1: Activation barriers, formation and dissociation energies for radical hydrogen transfer. Energies are reported in eV's. See Figure 2 for bond labels. 

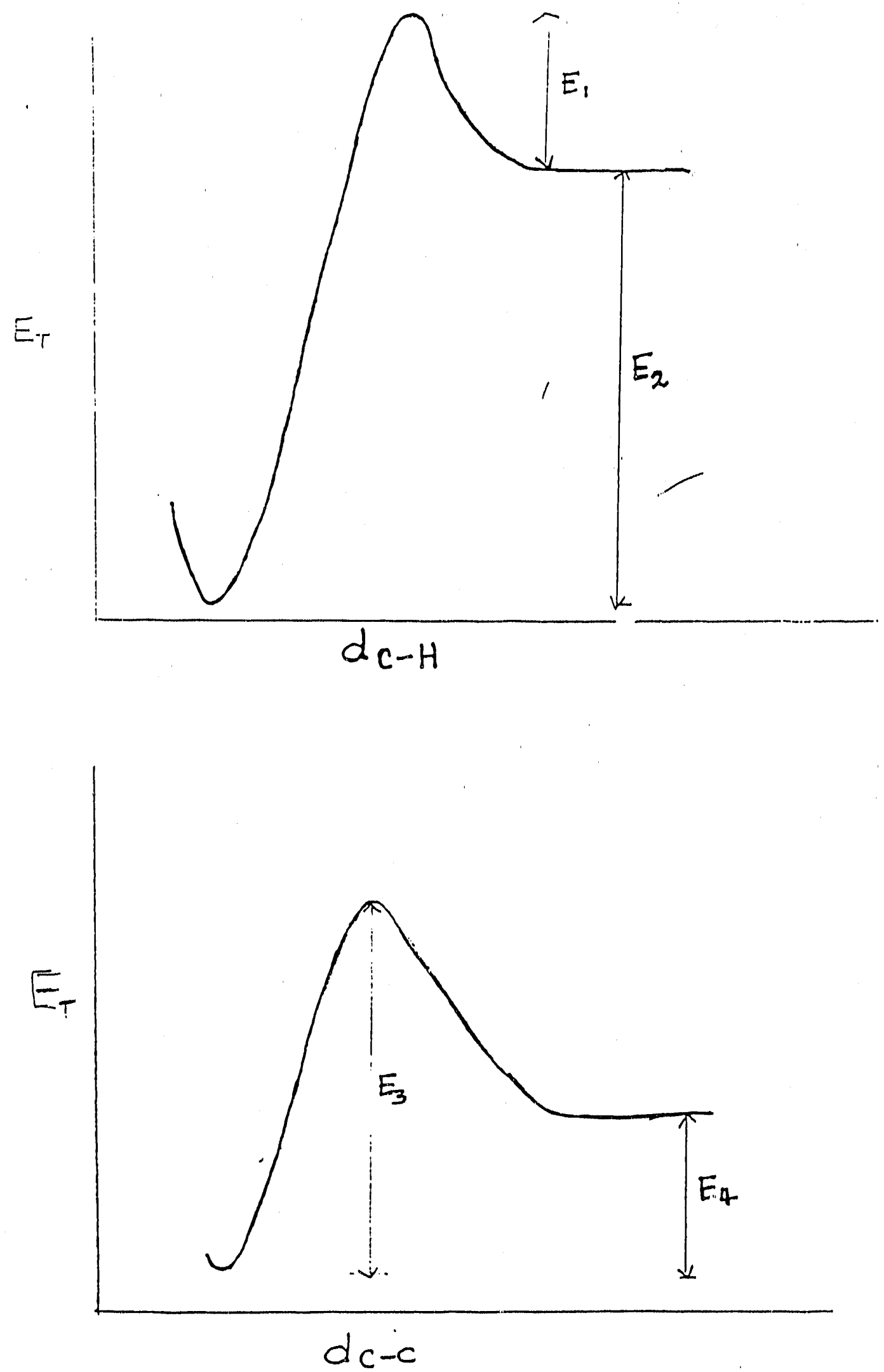

Figure 2: Hydrogen Addition and Dissociation Reaction Paths. 


\title{
Project II.7 Chemical Characterization and Hydrogenation Reactions of Single Coal Particles
}

\author{
Asit Ray \\ University of Kentucky
}

During this reporting period, we have modified our experimental systems and conducted experiments on physical and chemical characterization of single coal particles. Physical characterization involves determination of size and shape of single coal particles, and their abilities to adsorb various gases and vapor. Experiments were conducted in an electrodynamic balance ${ }^{1}$ mounted inside a vacuum chamber which can be evacuated to a pressure of $10^{-3}$ torr. The electrodynamic balance operates as a charge-to-mass ratio filter; depending on the frequency and amplitude of the applied a.c. potential, particles within a narrow band of charge-to-mass ratio can be stably contained inside the balance. The a.c. field creates a net time-averaged force which is directed towards the center of the balance. At the center, the time varying force vanishes. Therefore, a charged particle can be suspended free of perturbation for an unlimited time at the center by applying a d.c. potential across the endcap electrodes to balance the vertical (gravitational and drag) forces. In a stagnant environment, the balancing d.c. voltage is proportional to the mass of the particle. The mass change $\Delta \mathrm{m}$, due to adsorption of a gas or vapor is related to the change in the balancing voltage $\Delta V$, by

$$
\frac{\Delta \mathrm{m}}{m_{0}}=\frac{\Delta V}{V_{0}}
$$

where $m_{0}$ and $V_{0}$ are the initial mass and the corresponding balancing voltage, respectively. The accuracy of mass change determination depends on the precision with which the particle is kept at the center of the balance. We have developed an automatic feedback device based on a video camera to position and maintain a particle suspended precisely at the center of the balance. A schematic of the balancing scheme is shown in Figure 1. The video camera has 
$526 \times 526$ detector elements, separated by a distance of $22.5 \mu \mathrm{m}$. Light scattered by a particle at the center of the balance is focused on the detectors by a zoom lens in such a way that the image of the particle lies between two rows of detectors. The video signals from these two rows are compared and a correction voltage is generated. When the particle is exactly at the center, the two rows of detectors are illuminated equally, and produce identical video signals. We have tested the accuracy and sensitivity of the feed back control system by comparing the mass obtained from the balancing voltage data with the mass calculated from the size obtained from an optical resonance technique ${ }^{2}$. Figure 2 shows a comparison for an evaporating glycerol droplet. The optical resonances observed in the light scattering is used to obtain size as function of time with an accuracy of 1 part in $10^{6}$. The results show that the sensitivity of the balancing voltage method for the droplet was about $5.15 \times 10^{-2} \mathrm{ng} / \mathrm{volt}$, and the standard deviation between the masses calculated from the two methods was about $1.63 \times 10^{-17} \mathrm{~g}$. This corresponds to an accuracy of about 1 part in $10^{5}$ in the mass determination from the balancing voltage data. This sort of sensitivity and accuracy is critical for adsorption studies involving small coal particles. In our experiments, we also flowed a controlled gas stream around the particle. In such a situation, the balancing d.c. voltage is proportional to the difference between the gravitational and drag forces on the particle. Thus, we obtained the mass and aerodynamic particle size from the voltages require to suspend a coal particle in the stagnant and flow environments. We have used these techniques to analyze the ability of a coal particle to adsorb various gases. In these experiments, we suspended $5 \mu \mathrm{m}$ to $20 \mu \mathrm{m}$ size single coal particles in the chamber at one atmospheric pressure. Then, we slowly evacuated the chamber to a pressure of $10^{-3}$ torr. We monitored the mass change of a particle as a function of total pressure. Finally, we increased the total pressure by introducing desired gas or vapor, and re-evacuated the chamber. We have conducted experiments with helium, nitrogen and water vapor. Figures 3 and 4 shows adsorption and desorption isotherms for coal particles in atmospheres containing helium or water vapor. The results show that there is an hysteresis between the adsorption and desorption patterns. For a given particle, the percentage of adsorbed mass as a function of the total pressure shows high degree of reproducibility. However, at a given pressure the percentage of adsorbed mass varies widely from particle to particle. This behavior is probably 
related to the variation of porosity and shape of the particles used.

For chemical characterization we have used Raman spectroscopy. The intensity of inelastically (i.e., Raman) scattered light from a microdroplet is very low. Therefore, detections of Raman scattering signals from small particles are quite tricky. In the current experimental system for Raman spectroscopy of single particles, only a small fraction of the total scattered light at a given wavelength is received by the detector (e.g, photo-multiplier tube). Due to the weak signal level in the system, obtaining a complete spectrum of a particle requires more than one hour. Moreover, the use of an Optical Multi-channel Analyzer (OMA), where the scattered light over a range of wavelengths can be recorded simultaneously, is not possible. To overcome these difficulties, we have modified our experimental system where more than $80 \%$ of the total scattered light from a particle is received by the detector. A schematic of the experimental system is shown in Figure 5. A two ring electrodynamic balance is mounted inside a parabolic mirror. The null point of the balance where the suspended particle remains stationary for a prolonged period of time coincides with the focal point of the parabolic mirror. All the light scattered by the particle that hits the parabolic mirror is reflected down, forming a beam of collimated light. More of the scattered light is collected by placing a focusing lens at its focal length beneath the particle but above the large focusing lens shown in Figure 1. The parallel light rays are then focused by a lens and redirected by a flat mirror toward the entrance of a spectrometer (i.e., double-monochromator). A final focusing lens is used to focus the light precisely at the entrance slit of the spectrometer, and produce the correct $f$-ratio for maximizing the spectrometer performance. A chilled photomultiplier or an OMA is used to detect light at the exit of the spectrometer, and output signal is amplified for photon counting. Figure 6 shows a spectrum obtained from a $11 \mu \mathrm{m}$ coal particle. The spectrum was obtained in the range $400 \mathrm{~cm}^{-1} 4000 \mathrm{~cm}^{-1}$ in steps of $5 \mathrm{~cm}^{-1}$, using $500 \mathrm{~mW}$ power from an Ar-Ion laser (514.3). The spectral width was set at $5 \mathrm{~cm}^{-1}$, and the count time was $10 \mathrm{~s}$. The background counts without the particle in the presence of the laser beam varied from 10 to $20 \mathrm{cps}$. In the Raman active region the counts as high 1800 photons/s was observed. The signal to noise ratio was significantly high, indicating that the technique can be applied to much smaller particles. We are currently examining ways to obtain particle composition from Raman 
related to the variation of porosity and shape of the particles used.

For chemical characterization we have used Raman spectroscopy. The intensity of inelastically (i.e., Raman) scattered light from a microdroplet is very low. Therefore, detections of Raman scattering signals from small particles are quite tricky. In the current experimental system for Raman spectroscopy of single particles, only a small fraction of the total scattered light at a given wavelength is received by the detector (e.g, photo-multiplier tube). Due to the weak signal level in the system, obtaining a complete spectrum of a particle requires more than one hour. Moreover, the use of an Optical Multi-channel Analyzer (OMA), where the scattered light over a range of wavelengths can be recorded simultaneously, is not possible. To overcome these difficulties, we have modified our experimental system where more than $80 \%$ of the total scattered light from a particle is received by the detector. A schematic of the experimental system is shown in Figure 5. A two ring electrodynamic balance is mounted inside a parabolic mirror. The null point of the balance where the suspended particle remains stationary for a prolonged period of time coincides with the focal point of the parabolic mirror. All the light scattered by the particle that hits the parabolic mirror is reflected down, forming a beam of collimated light. More of the scattered light is collected by placing a focusing lens at its focal length beneath the particle but above the large focusing lens shown in Figure 1. The parallel light rays are then focused by a lens and redirected by a flat mirror toward the entrance of a spectrometer (i.e., double-monochromator). A final focusing lens is used to focus the light precisely at the entrance slit of the spectrometer, and produce the correct f-ratio for maximizing the spectrometer performance. A chilled photomultiplier or an OMA is used to detect light at the exit of the spectrometer, and output signal is amplified for photon counting. Figure 6 shows a spectrum obtained from a $11 \mu \mathrm{m}$ coal particle. The spectrum was obtained in the range $400 \mathrm{~cm}^{-1} 4000 \mathrm{~cm}^{-1}$ in steps of $5 \mathrm{~cm}^{-1}$, using $500 \mathrm{~mW}$ power from an Ar-Ion laser (514.3). The spectral width was set at $5 \mathrm{~cm}^{-1}$, and the count time was $10 \mathrm{~s}$. The background counts without the particle in the presence of the laser beam varied from 10 to $2.0 \mathrm{cps}$. In the Raman active region the counts as high 1800 photons/s was observed. The signal to noise ratio was significantly high, indicating that the technique can be applied to much smaller particles. We are currently examining ways to obtain particle composition from Raman 


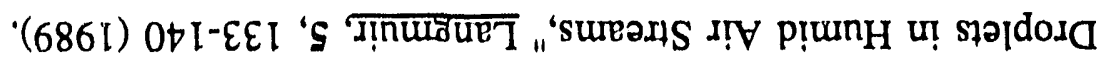

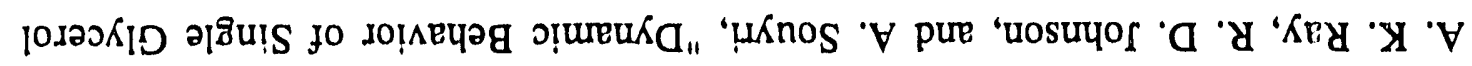

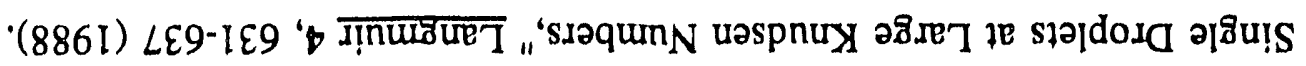

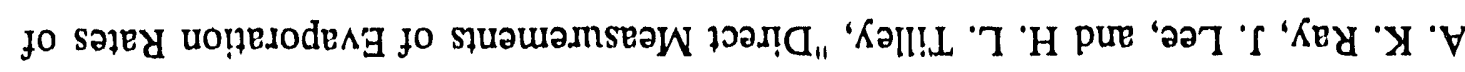


$\frac{\sum_{W}^{\omega}}{U}$

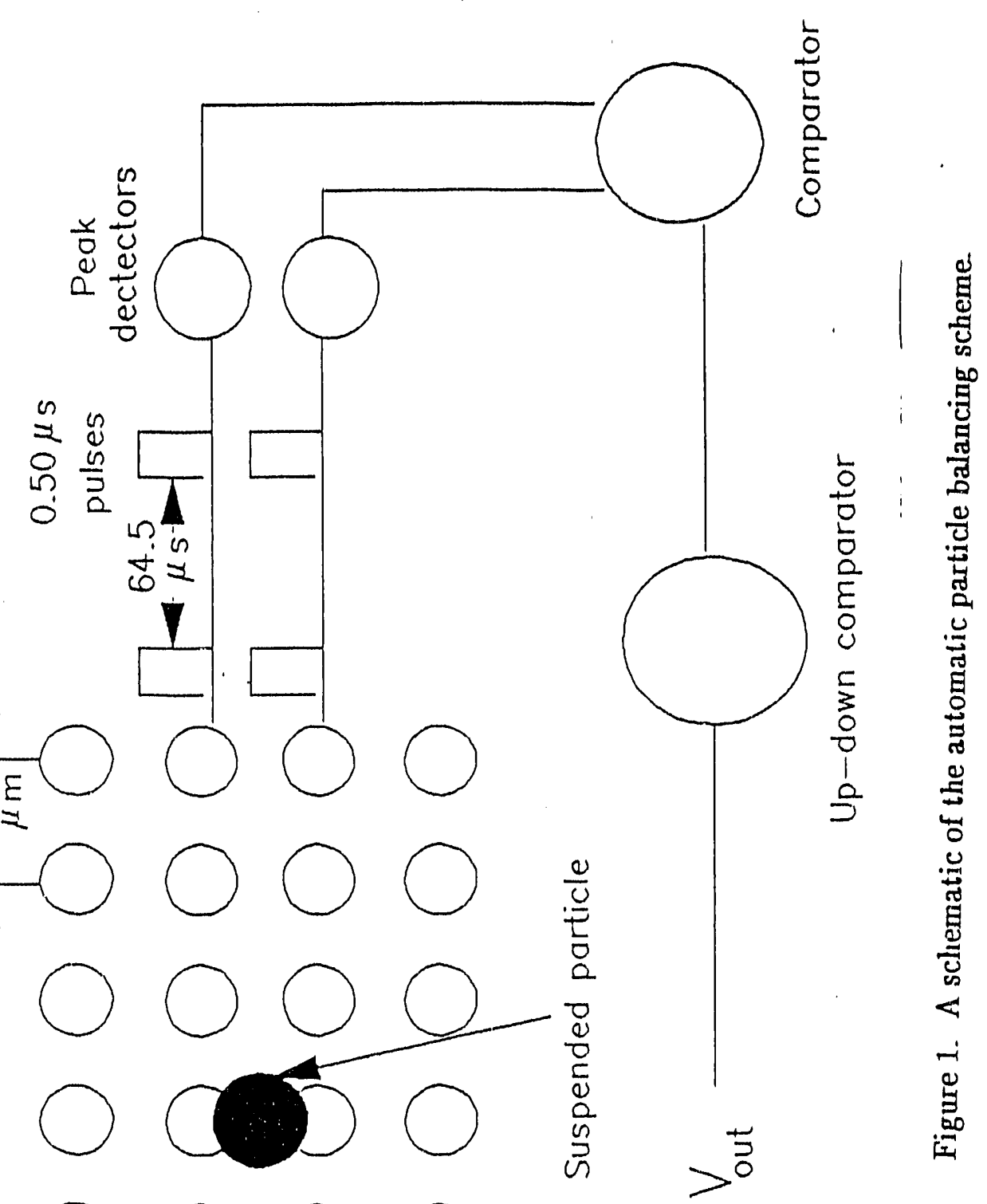

0
$\sum$
$\frac{Z}{Z}$

แ

$\circlearrowright$

$\frac{0}{0} 4$

$\cong \quad 0$

$\frac{\gamma}{1}$

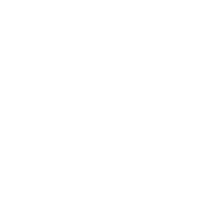

$\frac{1}{0}$
$\frac{0}{0}$
$\frac{0}{\varepsilon}$
0
0

范

巳 !

$\bigcirc$

0

0

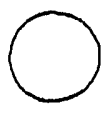

而 $\quad 0 \%$

0

(

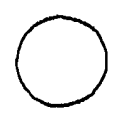

$\bigcirc$

$\bigcirc$

$\square$

$\square$ 


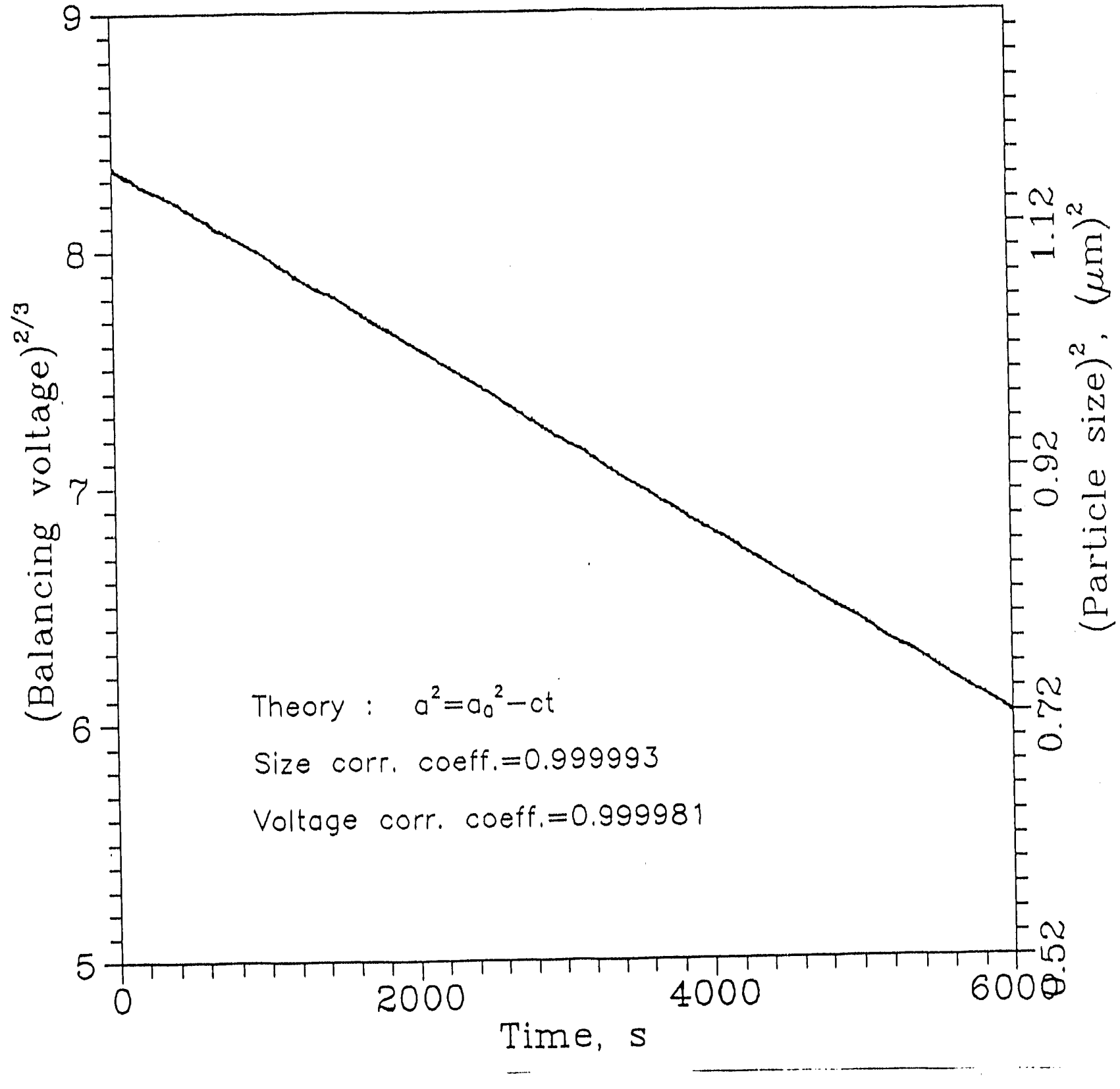

Figure 2. A comparison between mass of an evaporating glycerol droplet obtained from the balancing voltage data to that obtained from the optical resonance technique 


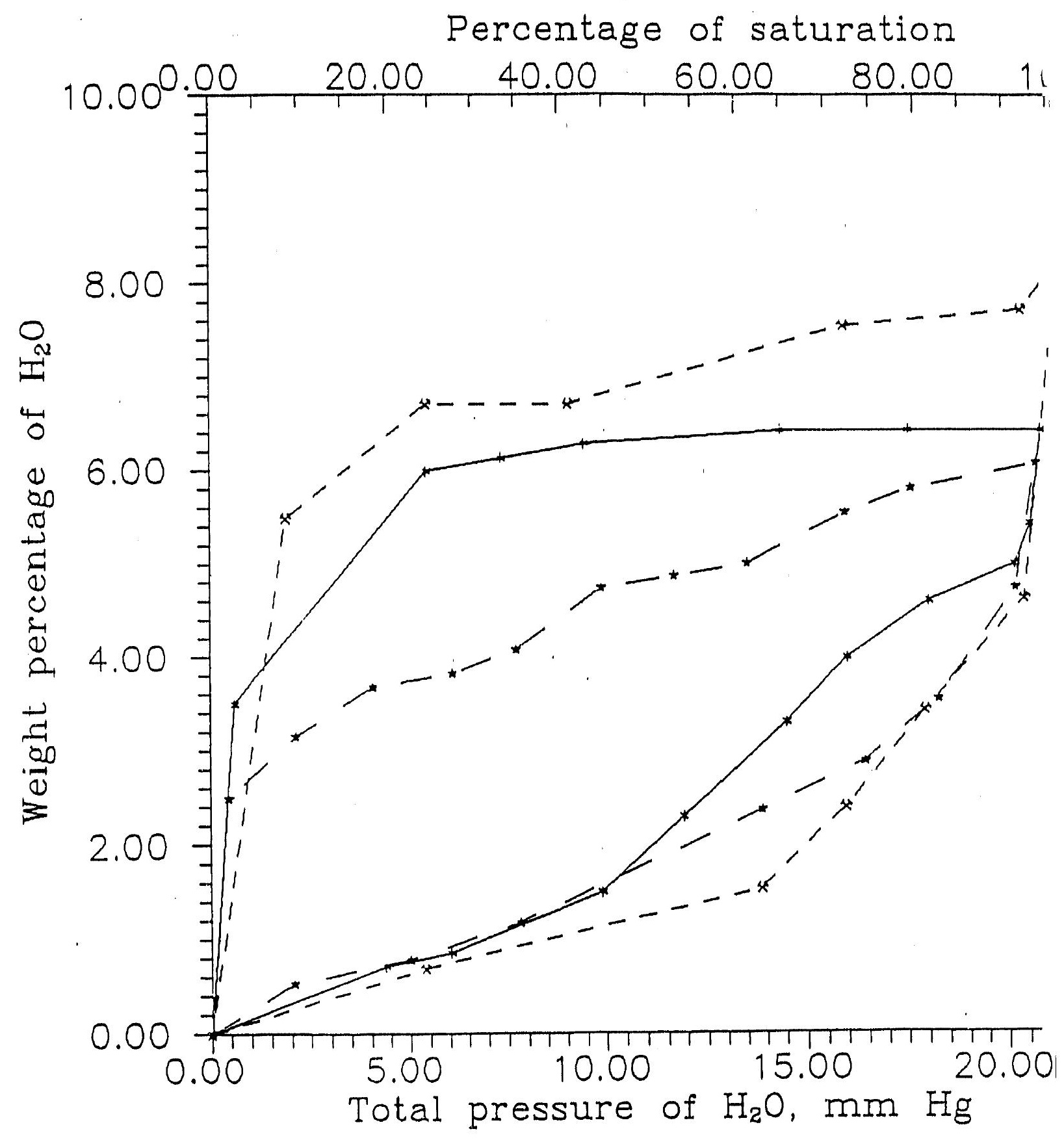

Figure 3. Adsorption and disorption isotherms of water vapor on coal particles. 


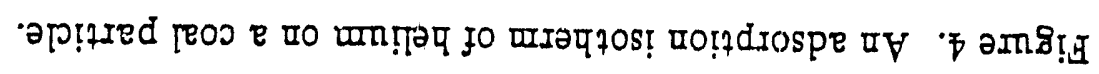

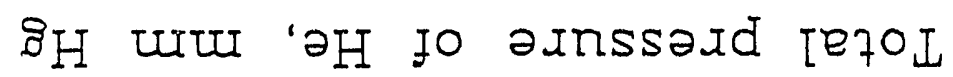

$\begin{array}{lllllll}30.0001 & 00.008 & 00.009 & 00.00 t & 00.002 & 00.0\end{array}$

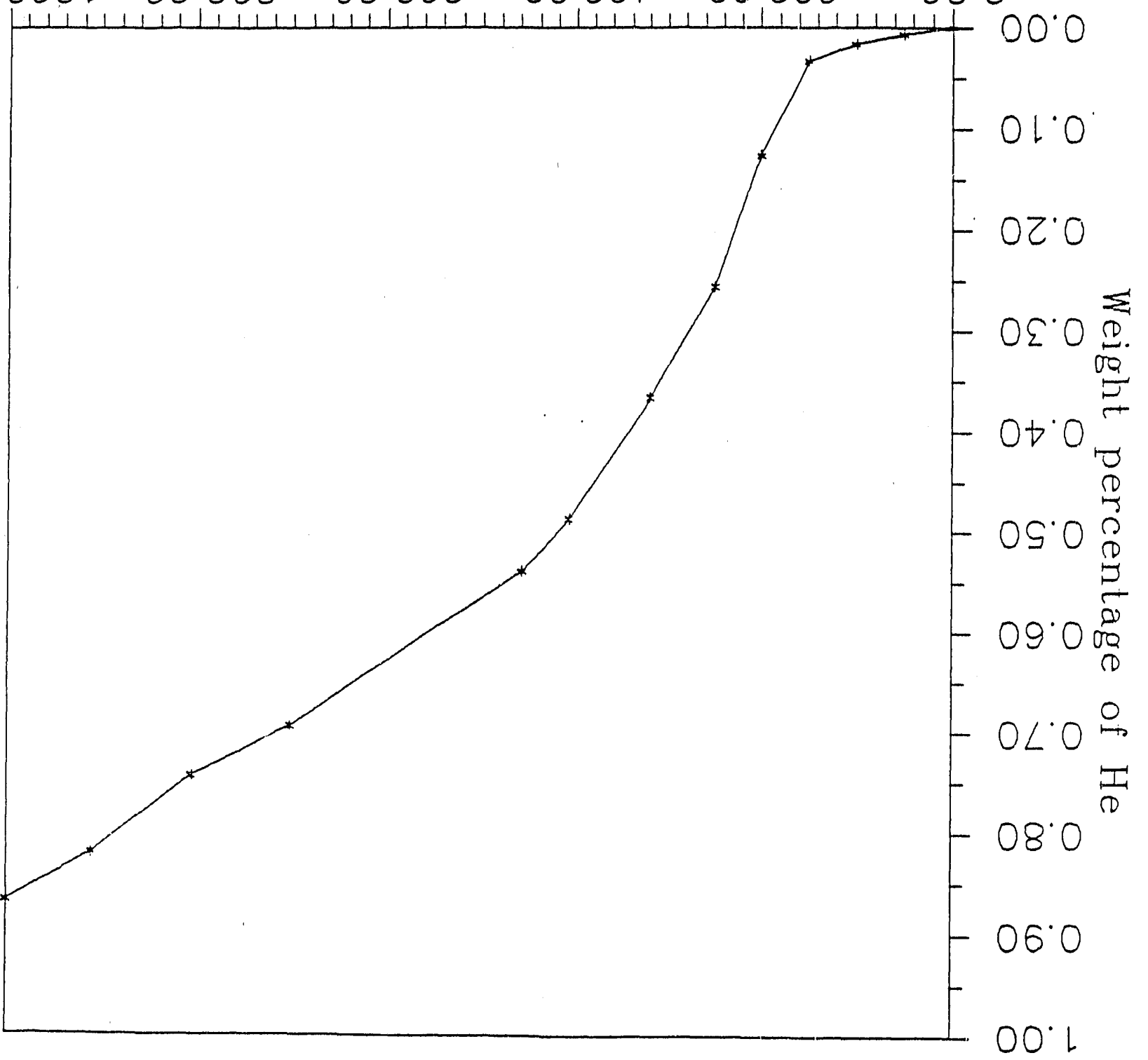




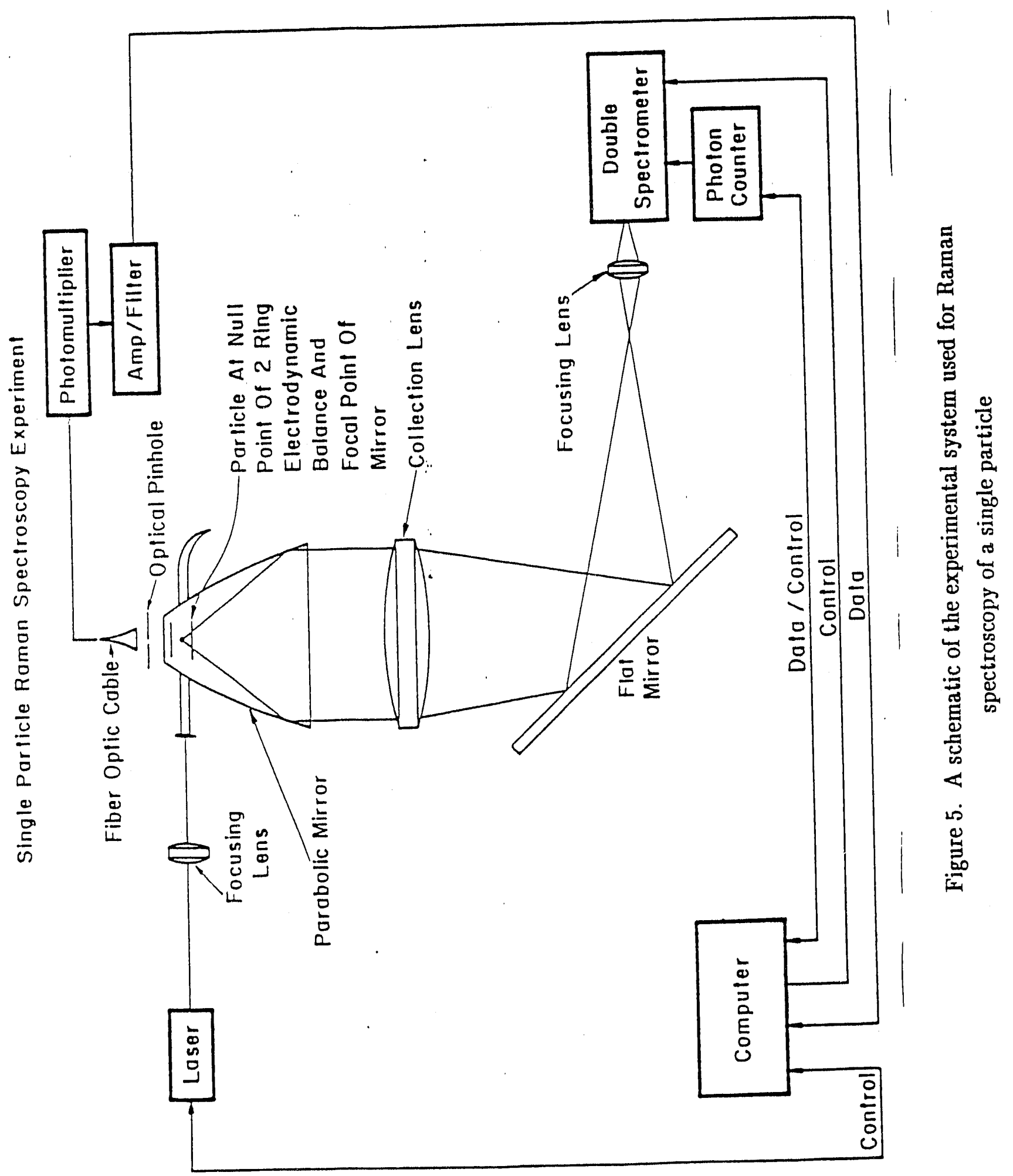




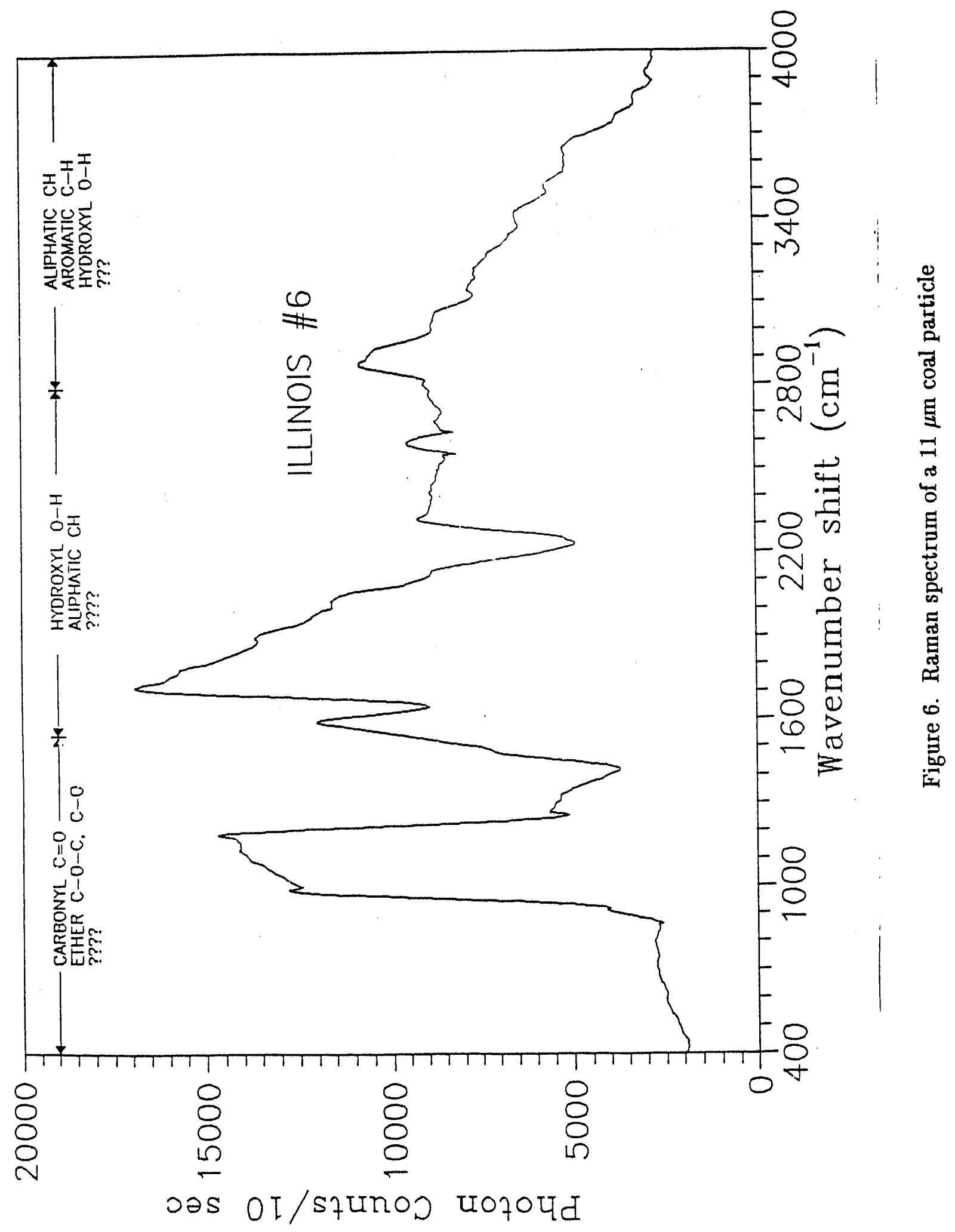




\title{
Project II.8 Liquefaction Pathways
}

\author{
B. H. Davis and R. A. Keogh \\ University of Kentucky - CAER
}

The thermal liquefaction data obtained for the 69 high volatile bituminous coals using a 15 minute residence time and three reaction temperatures $\left(385^{\circ} \mathrm{C}, 427^{\circ} \mathrm{C}, 445^{\circ} \mathrm{C}\right)$ suggest a common liquefaction pathway for these coals. A single bituminous coal (W.Ky. \#6) was selected to verify the suggested pathway. Liquefaction conditions were selected to produce a series of conversion levels which would define the pathway of the coal from a minimum conversion level to a maximum conversion level.

The thermal pathway of the Western Kentucky \#6 coal over the entire range of conversions is identical to that suggested in Figure 1 for the diverse set of coals. In the initial stage of dissolution, the primary reaction is the conversion of coal (IOM) to the $A+P$ intermediate. In this region of the pathway, the conversions show a positive linear correlation with the $A+P$ yields. It is also observed in this region of the pathway that the $O+G$ yields remain relatively constant. The pathway changes in the region of maximum conversion (and $A+P$ yield). In this section of the pathway, the primary reaction taking place is in the conversion of the intermediate, $A+P$, to the final product $O+G$. During this stage of the liquefaction pathway, conversion remains relatively constant. At the highest reaction temperature $\left(445^{\circ} \mathrm{C}\right)$ and long residence times $(>15 \mathrm{~min}$.), the pathway appears to change. In this region, conversion, $A+P$ and $O+G$ yields concurrently decrease, indicating the possibility that retrograde reactions are predominant. The results of these experiments suggest that the high volatile bituminous coals studied in this work have a common thermal liquefaction pathway.

The liquefaction pathway defined by these coals indicates that a maximum in the intermediate yields $(\mathrm{A}+\mathrm{P})$ must be achieved prior to an increase in the $\mathrm{O}+\mathrm{G}$ yields. It is desirable to alter the pathway in such a manner that the $O+G$ yields increases with increase in coal conversion. A number of catalysts were studied to determine if the added catalyst effect the selectivity defined by thesu :eactions and thus change the pathway. The catalytic 
pathway is compared to the thermal pathway using the Western Kentucky \#6 coal and two iron based catalysts. The addition of these catalysts had no effect on the observed thermal pathway. Identical experiments employing a supported catalyst (Shell 324), an oil soluble catalyst precursor (molybdenum naphthenate) and two acid catalysts $\left(\mathrm{ZnCl}_{2}\right.$ and $\left.\mathrm{SnCl}_{2} \cdot 2 \mathrm{H}_{2} \mathrm{O}\right)$ also produced the same pathway that was observed for the thermal experiments. Therefore, the effect of catalyst addition is to increase the rate of production of the intermediate, $A+P$. The addition of catalysts has no effect on the selectivity defined by the solubility classes.

The effectiveness of a catalyst may be influenced by the quality of the liquefaction solvent. To determine the effect of solvent quality, as measured by hydrogen donor ability, a series of experiments (catalytic and thermal) were conducted using a Western Kentucky \#9 coal and a donor (tetralin) and a non-donor (1-methylnaphthalene). These data show that no change was observed in the liquefaction pathway. Additional experiments which used a mixture of phenanthrene and hydrogenated phenanthrenes as the liquefaction solvent defined the same pathway described above.

The next task in this investigation was to determine if the catalytic and thermal pathways of low rank coals were the same as that defined by the high volatile bituminous coals. A Wyodak subbituminous coal was chosen for the initial thermal and catalytic $\left(\mathrm{Fe}_{2} \mathrm{O}_{3}\right.$, molybdenum naphthenate) experiments. The thermal and catalytic pathway are also identical for this coal. However, the pathway for the subbituminous coal is substantially different from the pathway defined by the bituminous coals. Similar to the pathway of the bituminous coal, there are two regions in the pathway of the subbituminous coal. During the initial coal dissolution stage, both the $\mathrm{A}+\mathrm{P}$ and $\mathrm{O}+\mathrm{G}$ yield increase with increasing coal conversion. The second region of this pathway is similar to that of the bituminous coal. Here the coal conversion increases minimally and the major reaction is the conversion of $A+P$ to $O+G$. On the basis of these solubility class data, the subbituminous coal has an improved catalytic and thermal pathway when compared to that of the bituminous coals studied due to the concurrent increase in $O+G$ yields with coal conversion.

The utilization of lumped solubility class parameters and the Wei-Prater diagram have shown that the bituminous coals studied have a common thermal liquefaction pathway. Attempts to change the observed pathway using a number of catalysts and liquefaction 
solvents were not successful. The role of the catalyst in this work is to increase the rates of the defined reactions. The catalyst provided no selectivity to the production of the final products, $\mathrm{O}+\mathrm{G}$.

The pathway defined by a subbituminous Wyodak coal was substantially different from the pathway of the bituminous coals. The major difference was the parallel increase in $A+P$ and $O+G$ yields with the increase in coal conversion. Similar to the bituminous coal data, addition of catalysts had no effect on the Wyodak thermally defined pathway.

The next task in this investigation of liquefaction pathways using the Wei-Prater method was to define the pathways of the asphaltene and preasphaltene intermediates. In order to obtain sufficient amounts of the intermediates, the CAER pilot plant was modified for this task. The reactor, a 1 liter stirred autoclave, was incorporated into the pilot plant feed system, gas delivery and liquid separator systems. The Western Kentucky \#9 coal was slurried (30 wt.\%) in an Ashland petroleum heavy oil. The petroleum heavy oil (100\% oils) was used as a solvent so that all of the asphaltenes and preasphaltenes obtained from the reaction were coal-derived. The reactor was run on a 24 hour basis and upon reaching process steady state for a particular set of conditions sampling of the product was initiated. Two reactor temperatures $\left(385^{\circ} \mathrm{C}\right.$ and $450^{\circ} \mathrm{C}$ ) and two residence times $(41 \mathrm{~min}$. and $26 \mathrm{~min}$.) were used in the liquefaction runs. In addition to these thermal runs, an oil soluble catalyst, molybdenum naphthenate, was injected prior to the reactor, at the end of each thermal run to provide intermediate from a catalytic reaction.

The asphaltenes and preasphaltenes were separated from the total liquid product using the standard CAER Soxhlet method. The initial separated asphaltenes and preasphaltenes required several solvent extractions to obtain the desired purity. The separation of the thermal asphaltenes obtained from the $385^{\circ} \mathrm{C}$ and $450^{\circ} \mathrm{C}$ reaction temperatures has been completed.

The asphaltenes were liquified in the batch microautoclave reactors using a number of residence times and reaction temperatures, hydrogen and tetralin as the solvent. Standard Soxhlet extraction were done for the reactor products. The solubility class distribution were lumped into the following parameters: (a) oils + gases, (b) asphaltenes (starting material), and (c) preasphaltenes + IOM. The lumped parameters were plotted on a Wei-Prater diagram as shown in Figure 1. 
The reaction pathway of the asphaltenes $\left(450^{\circ} \mathrm{C}\right)$ is exactly what was predicted from the data derived for the coal liquefaction path. The primary reaction is the conversion of the asphaltenes to oils + gases. As with the coal pathway, the primary component of the $\mathrm{O}+\mathrm{G}$ parameter is the oil solubility class. The gases average approximately $4 \mathrm{wt} . \%$ of the total lumped parameter. Retrogressive asphaltene reactions to preasphaltenes + IOM account for only a minor fraction of the conversion. The primary component of the preasphaltene + IOM parameter was preasphaltenes. Very little, if any, within experimental error, IOM was produced from the thermal conversion of the asphaltenes. A mixture of $70 \mathrm{wt} . \%$ asphaltene $\left(450^{\circ} \mathrm{C}\right)$ and $30 \mathrm{wt} . \%$ oil was also studied. There was no significant difference in the observed thermal pathway.

Also shown in Figure 1 are the results obtained from the thermal liquefaction experiments using the asphaltenes derived from the $385^{\circ} \mathrm{C}$ reaction temperature (CSTR). The thermal pathway for these asphaltenes appears to be slightly different than that observed for the $450^{\circ} \mathrm{C}$ asphaltenes. In the initial dissolution stage, the data suggests that the retrogressive reactions and the production of oils account for approximately equal fractions of the asphaltene conversion. However, after this initial dissolution stage, the primary reaction is the production of oils. The reason for the increase is observed retrogressive reactions for the $385^{\circ} \mathrm{C}$ asphaltenes may be related to their increase in reactivity when compared to the $450^{\circ} \mathrm{C}$ asphaltenes. The catalytic runs using both asphaltenes may give credibility to this suggestion. 


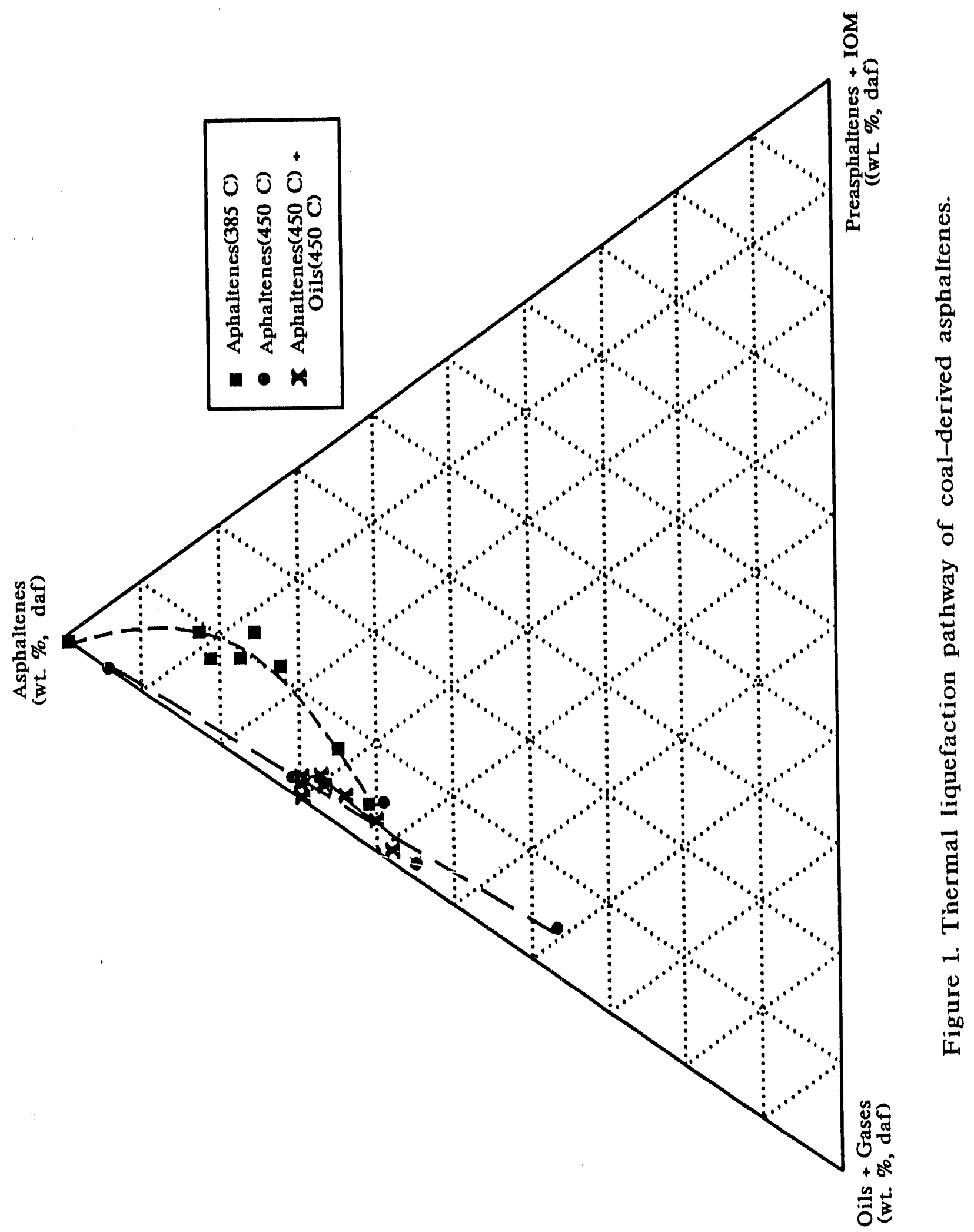




\title{
Project II.9 Development of Advance Methods of Coal Liquefaction Product Analysis
}

\author{
Edwin L. Kugler \\ Chemical Engineering Department \\ West Virginia University
}

\section{OBJECTIVES}

This research program has the goal of developing HPLC methods and techniques to separate coal liquids by hydrocarbon type. These separations would initially classify coal liquids by their content of saturate, aromatic and polar hydrocarbons. The aromatic fraction would be subdivided by aromatic ring size to distinguish one ring aromatics from others with two, three, four, five and more rings; the polar fraction would be separated by nitrogen and oxygen functional group. This classification should provide information necessary to evaluate and understand coal liquefaction process chemistry.

\section{ACCOMPLISHMENTS}

Since the outset of this project on May 1, we have worked on separating the oil fraction of solvent separated coal liquids into saturate, aromatic and polar molecules. This is a difficult separation, in that HPLC columns that separate saturates and aromatics often irreversibly adsorb polar molecules so that they do not come off the column. On the other hand, columns that elute polar molecules do not resolve saturates and aromatics into their components so that all the saturates and aromatics come off the column in a single peak. We solved this problem by using several columns and a column switching valve (1).

In our procedure, the sample is introduced into an HPLC system pumping a hexane solvent anci is initially separated on a DIOL column that retains polu's and passes saturates and aromatics. The saturates ana aromatics proceed through a column switching valve and into a DNAP column. At this point, the column switching valve is actuated, trapping the saturate and aromatic molecules on the DNAP column when it is isolated from the flow 
stream. Solvent flow continues through the DIOL column. The polar molecules that were initially retained on the DIOL column are now separated by introducing a methylene chloride gradient to the solvent stream. The solvent change from $100 \%$ hexane to $100 \%$ methylene chloride elutes most of the polar molecules. The remaining polars are desorbed from the column by adding $10 \%$ isopropyl alcohol to the methylene chloride stream.

After all the polar molecules have eluted from the DIOL column, the solvent is changed back to hexane and the column-switching valve is actuated to bring the DNAP column back into the flow stream. The saturates come off the DNAP column first, followed by aromatics separated by ring size as methylene chloride is blended into the hexane solvent.

This sequence, using three solvents, two types of column and a column switching valve separates a single injection of oil sample by molecular type, and further separates its aromatic molecules by ring size. These separations using ASTM open column methods require several days work. With the HPLC approach, similar separations are achieved in just over one hour.

Our initial HPLC separations using coal liquids from iron catalyzed Pittsburgh No. 8 coal showed that the oil fraction contained saturates, polars and aromatics with ring sizes from 3 to perhaps 8 rings (1). The later molecules had retention times significantly longer than our calibration standards with 1 to 5 aromatic rings. The asphaltene fraction of this same coal liquid sample contained only polar molecules, with no saturates or aromatics (1). The polar fractions from both the oils and asphaltenes have characteristic shapes, but to date, individual components in these fractions have nut been identified.

\section{IMMEDIATE PLANS}

Research is currently directed toward identifying components in the saturate, aromatic and polar fractions. The strategy being employed is to calibrate retention times with known standards and compare oil samples with data on pure compounds. Quantitation is planned based on collecting fractions of eluted samples and running analyses using a gas chromatograph with a flame ionization detector. The FID has good linearity for most hydrocarbon molecules so that this approach should allow us to obtain quantitative results and establish mass balances for our separation method. In addition, gas chromatography will 
provide further information on molecular size and structure.

\section{PERSONNEL}

This program was begun with an undergraduate student, Scott Kugler, employed for the summer only. With the beginning of the Fall semester, David Keely has joined the project as a graduate research assistant.

\section{REFERENCES}

1. E. L. Kugler and S. L. Kugler, CFFLS Program Review, Lexington, Kentucky, August 11-14, 1991 

$\cdot$ -
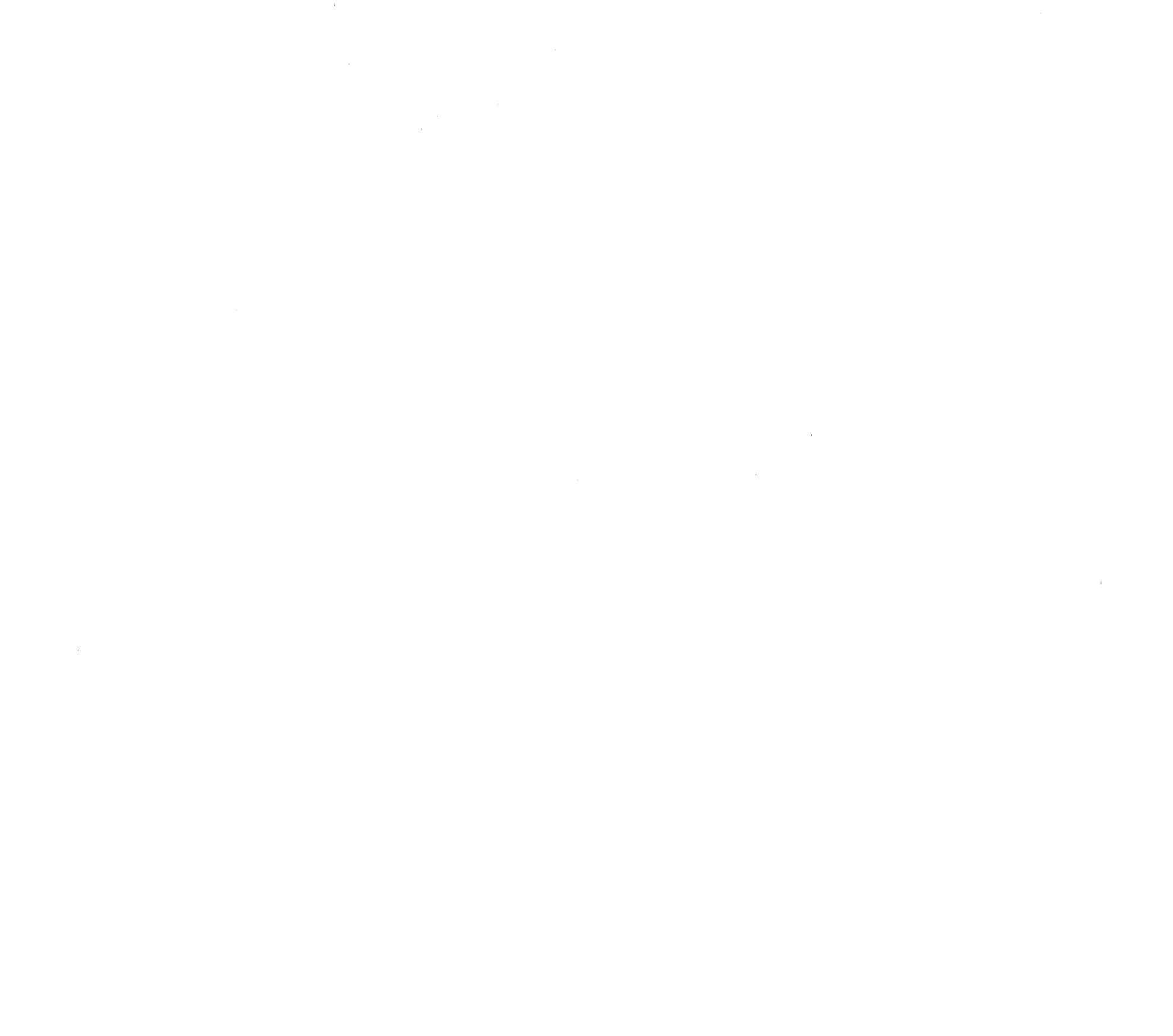


\section{Task III}

Novel Coal Liquefaction Concepts

Program Coordinators: D. Bhattacharyya, A.R. Tarrer, and J.S.Shabtai 


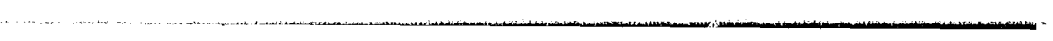




\title{
Project III.1 Bioprocessing of Coal
}

\author{
D. Bhattacharyya, M.V.S. Murty, R.I. Kermode, \\ M. Venkatachalam, M.I.H. Aleem, K. Overstreet \\ University of Kentucky
}

\section{SUMMARY}

In-situ catalyst formation studies with Sulfolobus brierleyi showed the formation of $\mathrm{FeOOH}$ crystals as well as impregnation of molybdenum on coal particles by various physical techniques. Our previous liquefaction yield results of the coal sample (KCER \# 91182) showed only $3 \%$ increase. The chemical liquefaction yield of the biotreated coal was dramatically increased by $26 \%$ when the coal sample containing bioformed catalyst was presulfided in the liquefaction reactor.

We have investigated the potential of hydrogenase-containing bacteria in the direct hydrogenation of different untreated coals and coal-related model compounds for improved liquefaction. Hydrogen uptake hydrogenase-possessing thermoacidophilic archaebacterium Sulfolobus brierleyi and mesophilic sulfate-reducing bacteria Desulfovibrio desulfuricans were used to study the biological hydrogenation of different coals and various model compounds such as diphenyl methane (DPM), 1,2-diquinolyl ethane, a-naphthyl hexane. Hydrogen uptake by the model compounds and various coal types catalyzed by the microbial systems was also determined using G.C. The experiments with the model compounds indeed showed hydrogen uptake ranging from $0.28 \mu$ moles $\mathrm{H}_{2} / \mu$ mole of DPM to $6.55 \mu$ moles $\mathrm{H}_{2} / \mu$ moles of 1,2-diquinolyl ethane in the presence of $S$. brierleyi and $D$. desulfuricans respectively. Chloroform extract of aqueous phase analysis using GC-MS did show that DPM was fragmented into lower parent fragments of $M / Z$ values 73,95 and 147 depending upon the microorganism used. We also observed significant spectral variation of the liquid phase of medium with coal (KCER \# 4677 91182) and asphaltene, when treated with S. brierleyi and $D$. desulfuricans under $\mathrm{H}_{2}$. Our previous results in fact showed hydrogen uptake by these 
coals and asphaltene.

\section{In-situ formation of fine $\mathrm{FeOOH}$ crystals:}

Coal (KCER \# 91182) was treated with Sulfolobus brierleyi at $60^{\circ} \mathrm{C}, \mathrm{pH} 2.5$, in presence of $\mathrm{CO}_{2}$, air and low concentration (200 ppm) of molybdenum salt. An experiment was conducted with the same parameters but in the absence of Sulfolobus brierleyi which is termed the control run. Two sets of experiments with $5 \%$ coal were treated with Sulfolobus brierleyi. In the shaker setup about $200 \mathrm{ppm}$ of molybdenum salt was added and the initial $\mathrm{pH} 3$ dropped to 2.4 during the course of the experiment. In the fermenter, $300 \mathrm{ppm}$ of molybdenum salt was added with $\mathrm{pH} 3$ was maintained. Both the experiments were conducted at $60^{\circ} \mathrm{C}$. Samples were taken every third day, filtered, washed and the coal was dried in an oven at $80^{\circ} \mathrm{C}$ for about 6 hours. Our previous findings have shown that Sulfolobus brierleyi could tolerate different amounts of molybdenum which was present in culture medium. Though there was an increase in protein content in culture broth, we observed a sudden decline of growth at the end of 12th day of the experiment.

\section{Mösşbauer Spectroscopy}

Mössbauer Spectroscopy provided a quantitative measurement of the reactions of pyrite and its transformations in Western Kentucky \# 11 coal treated for biodesulfurization by Sulfolobus brierleyi. Mössbauer analysis of these biotreated coal samples obtained at the end of the run showed significant changes in the iron forms (Table 1).

\section{XAFS}

XAFS study of biotreated samples obtained with increase of time exhibited prominent increase of peak between 50-60 eV represents Mo impregnation on coal (Fig.1). 
Elemental analysis on the surface of the biotreated cnal (KCER \# 91182) using X-ray photoelectron spectroscopic technique.

$\mathrm{X}$-ray photoelectron spectroscopic studies were conducted to note the surface characteristics of the biotreated and raw coal (KCER \# 91182). By this technique we determined the elements and chemical information about the outermost $100 \AA$ of the exposed coal surface. The elemental surface concentration determined by XPS was similar to the bulk analysis. From the results it was observed that the concentration of iron, molybdenum and sulfur (Fig. 2) of the fermenter treated coal were greater which is noted from the fact that the Fe 2p peaks are hard to detect in the case of raw coal (Fig. 3), whereas the peaks were distinct in the case of the fermenter treated coal. Raw coal had no molybdenum peaks and comparing the control runs (treated with no bacteria) experiments, the surface concentrations of Mo and Fe were found to be more in the test runs (Table 2).

The distinct $\mathrm{Fe}$ (2p3/2) peak (Fig. 2) in the range of binding energy 710-712 eV shows the iron is in the form of $\mathrm{FeOOH}$ (binding energy $710.7 \mathrm{eV}$ ) or Fe3O4 (binding energy 711.2 $\mathrm{eV}$ ). Similarly comparing with the standard peak of $\mathrm{MoO} 3$ (binding energy of $232.6 \mathrm{eV}$ ), we conclude that $\mathrm{Mo}$ is in the oxide state. The sulfur $2 \mathrm{p}$ peaks are visible at the binding energy range of $160-170 \mathrm{eV}$. Due to the oxygen effect the iron peak is shifted from standard binding energy of $161.5 \mathrm{eV}$ to the binding energy of $168.5 \mathrm{eV}$. Since the fermenter runs were conducted with continuous bubbling of $\mathrm{CO} 2$ and air we can see the sulfate peaks are greater in intensity. Also this explains the greater concentration of oxygen. Table 2 gives the normalized mass $\%$ of $\mathrm{C}, \mathrm{O} 2, \mathrm{~S}, \mathrm{Mo}$, and Fe present on the coal surface.

\section{Liquefaction of bioprocessed coal (KCER \# 91182) in the presence of H2S}

Liquefaction was conducted on both the samples of coal at $385^{\circ} \mathrm{C}, 800$ p.s.i. $\mathrm{H}_{2}$ for 15 minutes. Dimethyl disulfide (1 gram) was added to sulfide the iron and molybdenum present on the surface of the coal. The products of liquefaction contained benzene solubles which constitute the oils and asphaltene, and pyridine solubles benzene insolubles, which are the preasphaltenes. Oils and asphaltenes are separated by pentane which dissolves the oil leaving behind the preasphaltenes. The sulfided forms of iron and molybdenum act as 
catalysts during liquefaction. The total conversion determined as the pyridine solubles showed a substantial increase of $26 \%$ in the test run coal compared to the control run (Table 3, Fig.4). The experiments were repeated and the results obtained matched with the previous result. So far, this is the highest liquefaction obtained from any of the biotreated coals. Our previous liquefaction yield results showed only $3 \%$ increase without pre-sulfiding the bioprocessed coal. The interesting part of this work was that the reprecipitation of aqueous phase iron as $\mathrm{FeOOH}$ released from coal and sudden decrease of protein content of the culture broth might have influenced the control of the ultra-fine size of the catalyst.

\section{Hydrogenation of model compounds and coal by different bacteria}

Hydrogen uptake hydrogenase-possessing thermoacidophilic archaebacterium Sulfolobus brierleyi and the mesophilic sulfate-reducing bacteria Desulfovibrio desulfuricans were used to determine the biological hydrogenation of different coals and various model compounds such as diphenyl methane (DPM), 1,2-diquinolyl ethane, and a-naphthyl hexane. Washed cell suspensions were used from the $S$. brierleyi grown chemolithotrophically under aerobic conditions with elemental sulfur as the oxidizable energy source, and $D$. desulfuricans grown anaerobically with lactate as the energy source and sulfate as the final electron acceptor.

\section{Hydrogen uptake measurement by Gas Chromatography}

Coal samples were added to $160 \mathrm{ml}$ capacity serum vials containing $50 \mathrm{ml}$ of the mineral salts medium without the growth substrate. The sealed sterile vials were flushed with pure hydrogen gas and incubated at $37^{\circ} \mathrm{C}$ with $\mathrm{D}$. desulfuricans and at $60^{\circ} \mathrm{C}$ with S. brierleyi in the PsycrothermTM Controlled Environment Incubator Shaker. After allowing $30 \mathrm{~min}$. stabilization time $2 \mathrm{ml}$ of cell suspension ( $4.52 \mathrm{mg}$ cell protein/vial) was added only to the test vials. Gas samples were taken periodically using Unimetrics gas-tight syringe and analyzed by GC-9A (Shimadzu) attached with data processor Chromatopac C-R 1 B. The experiments with model compounds indeed showed hydrogen uptake ranging from $0.28 \mu$ moles $\mathrm{H}_{2} / \mu$ mole of DPM to 6.55 moles $\mathrm{H}_{2} / \mu$ moles of 1,2-diquinolyl ethane in the presence of S. brierleyi and D. desulfuricans respectively (Fig. 5-7). When 1,2-diquinolyl ethane was dissolved in 


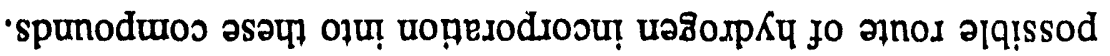

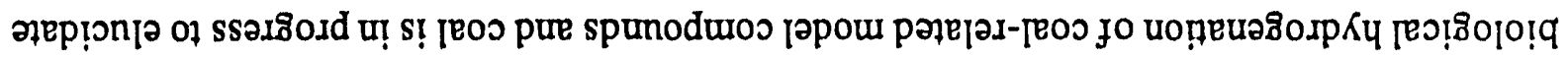

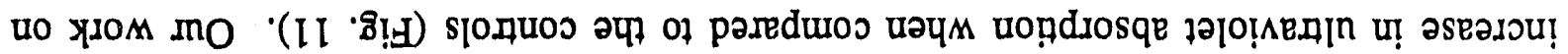

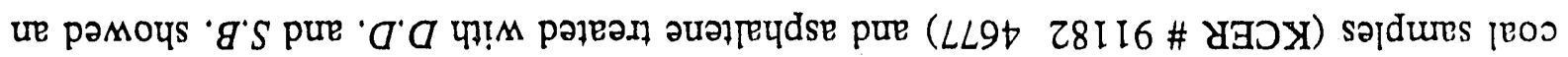

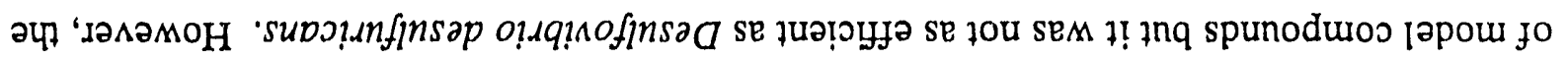

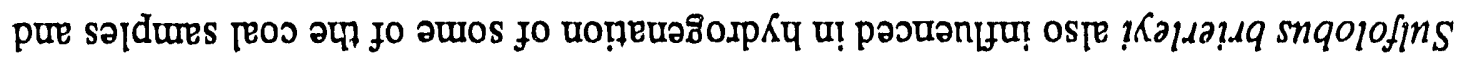

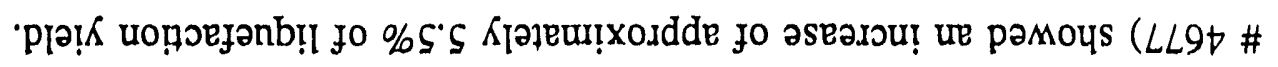

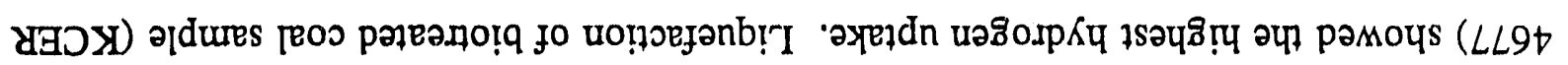

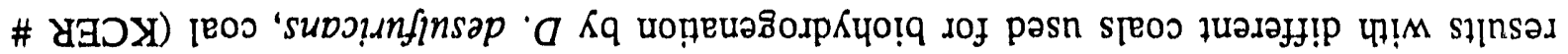

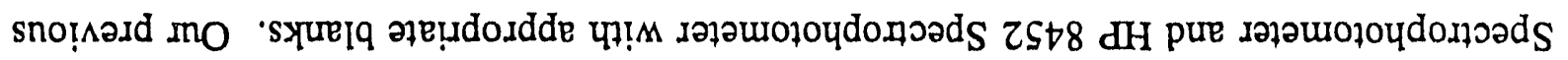

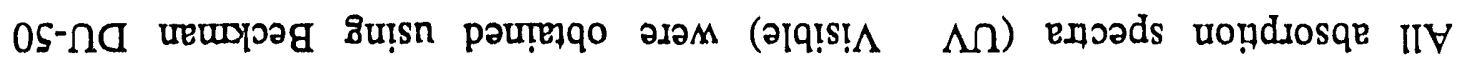

\section{S.lวumołoydodpordS}

$\cdot(01-8 \cdot 8 ! 7)$ pasn

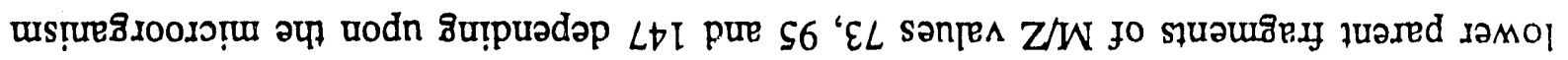

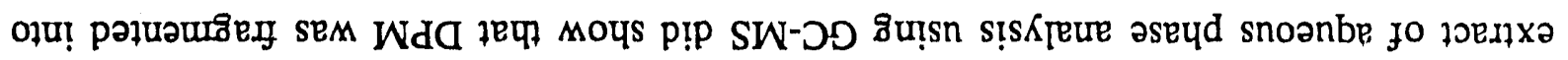

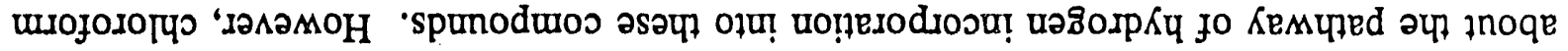

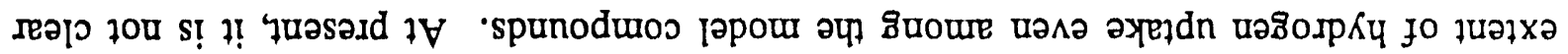

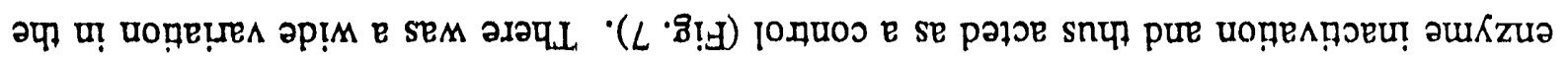

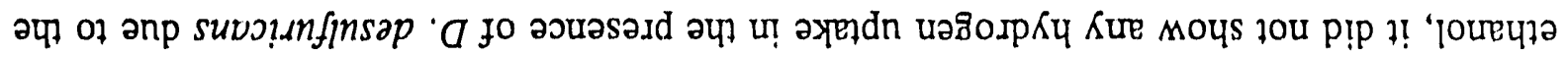




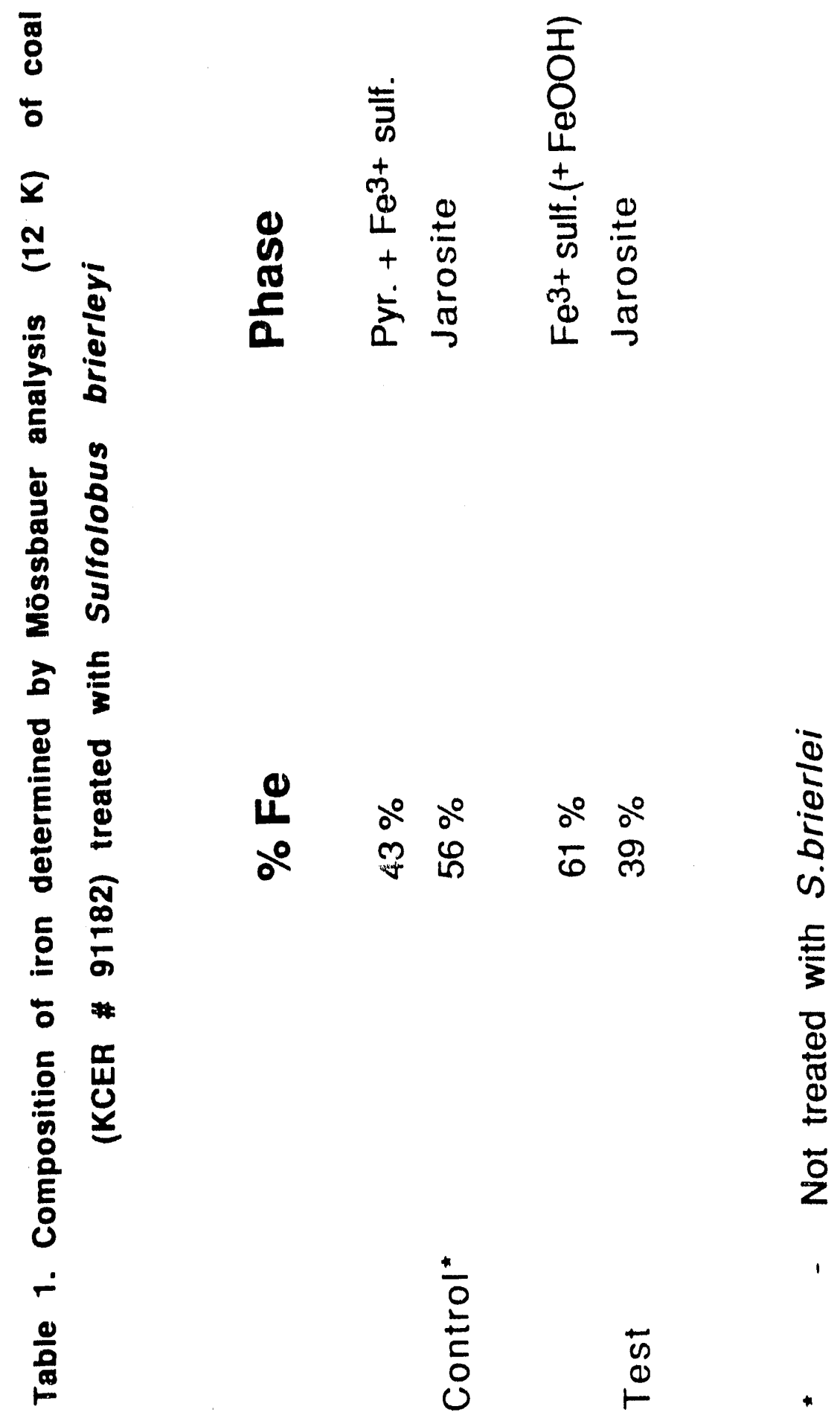



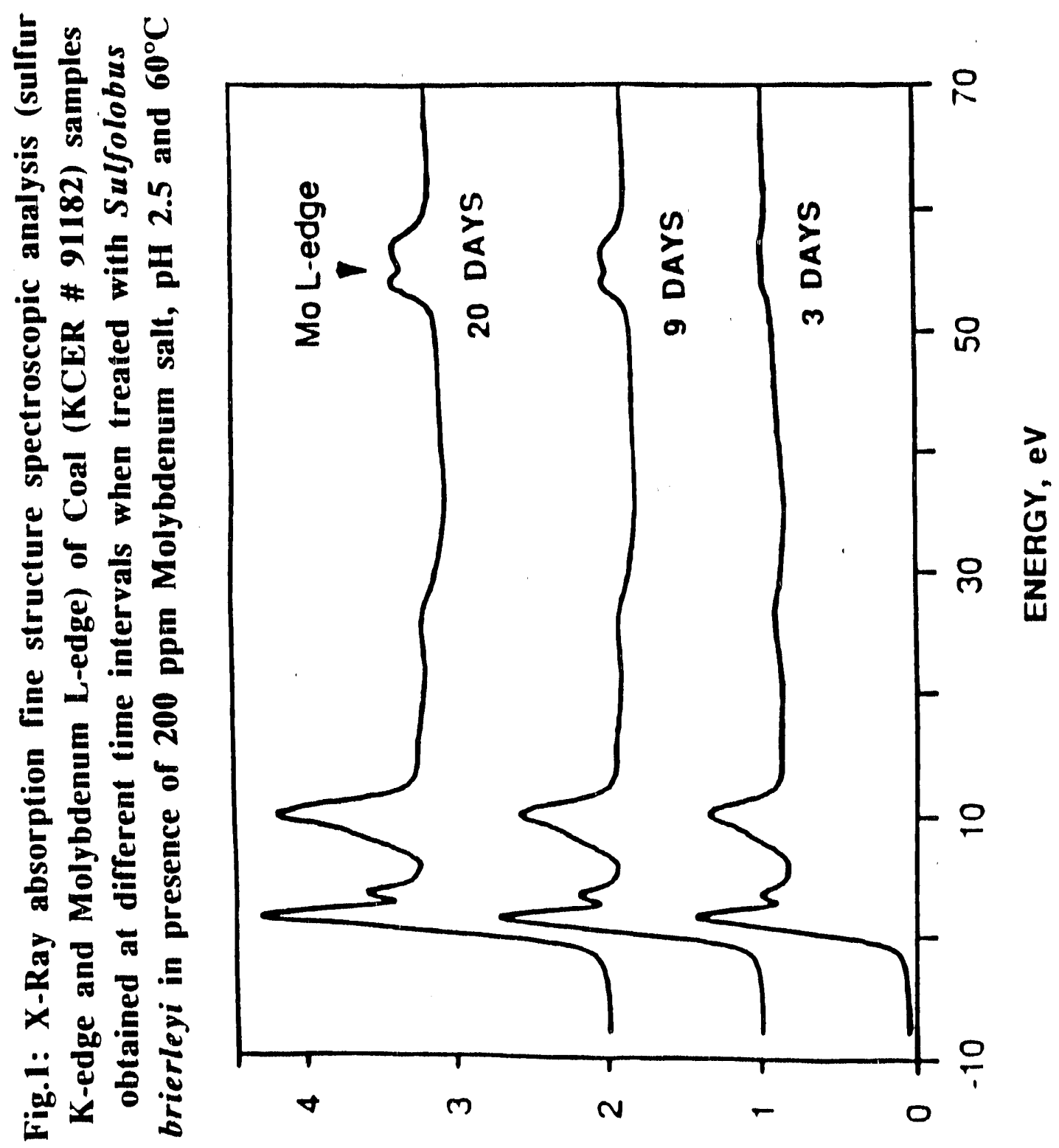

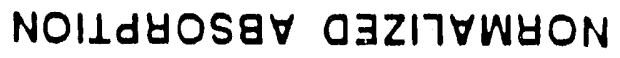



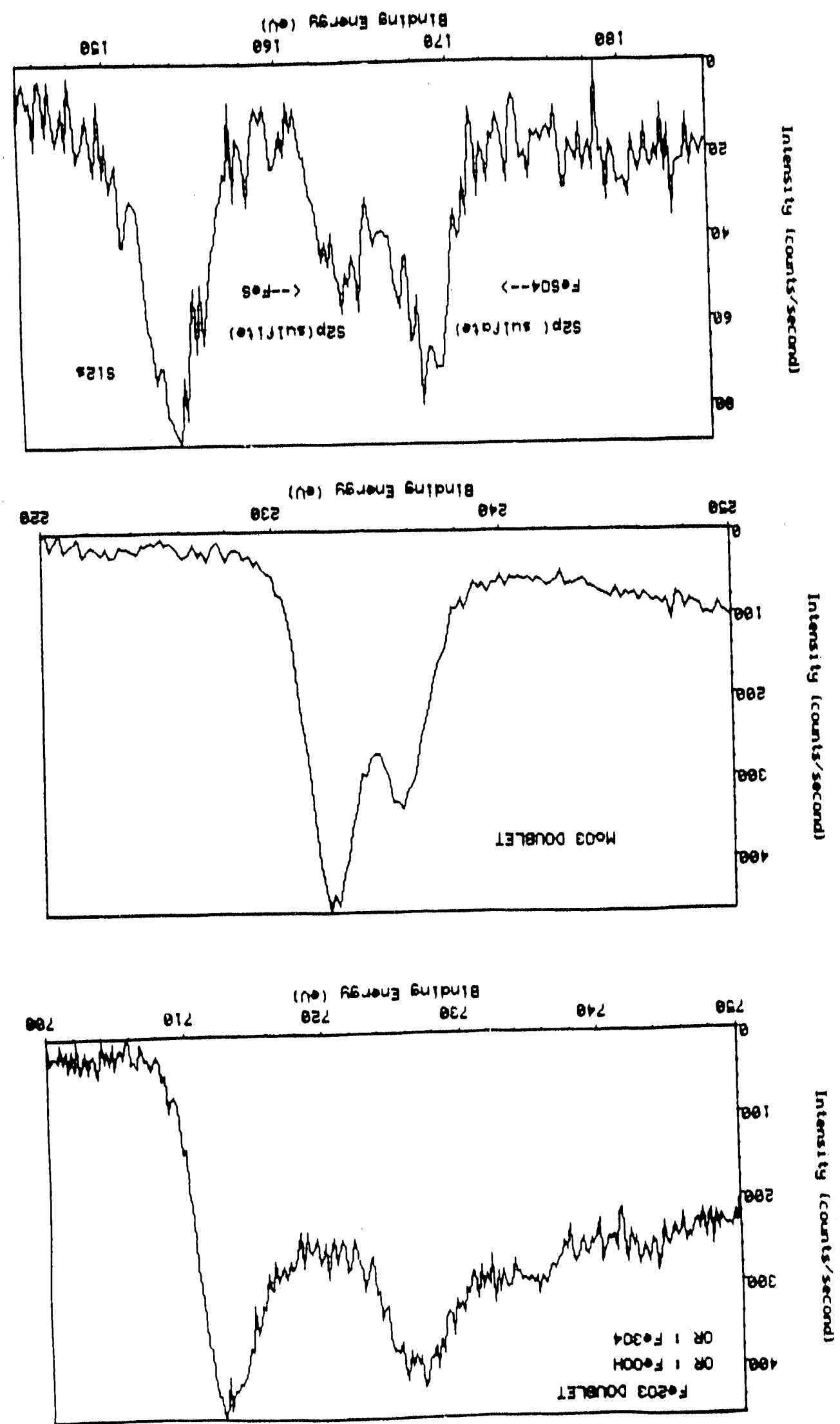

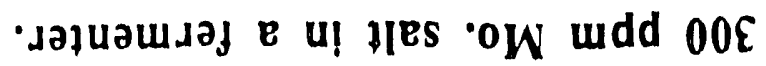

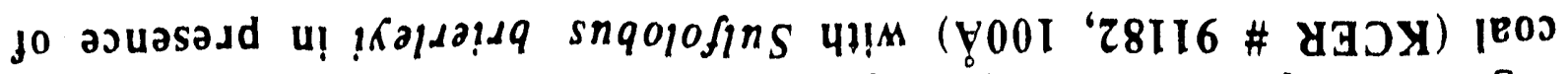

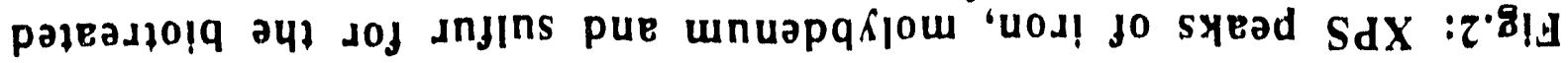



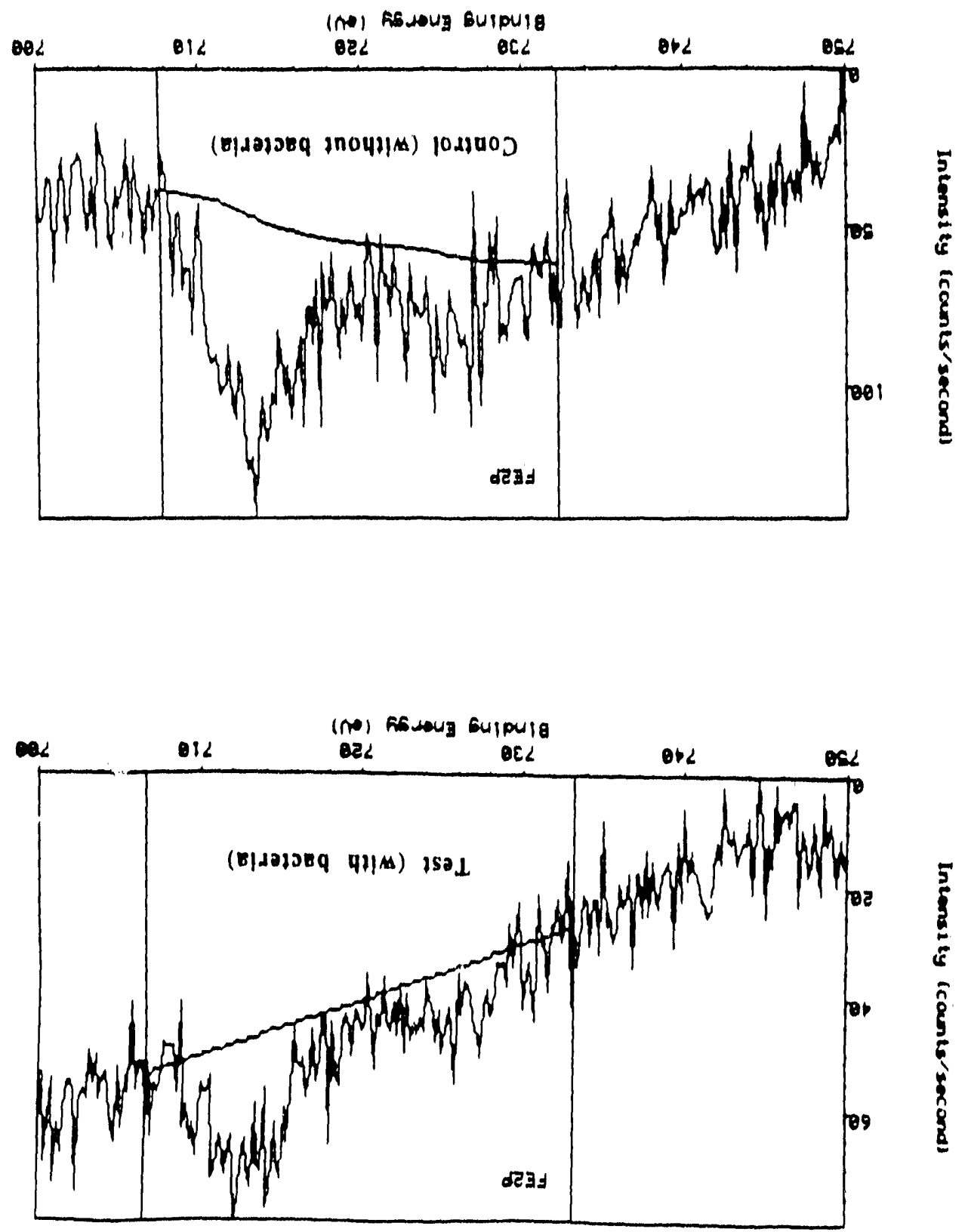

dnjas dayeus e u! hes 'oW undd $00 z$ jo ajuasadd

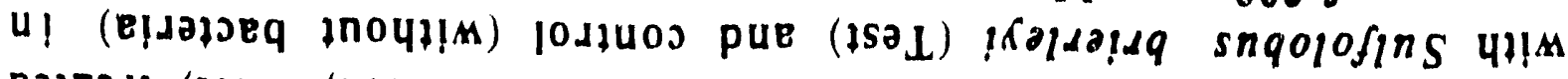
pəleast ( 
롤

5

롱

릉

6

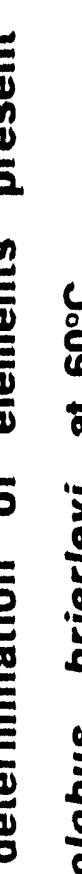

:

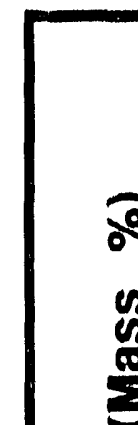

$\longrightarrow$

u.

\begin{tabular}{ll|l|l|l|}
$n$ & $\infty$ & $m$ & $m$ & $m$ \\
0 & $a$ & $n$ & $m$ & $a$ \\
\hline & $n$ & $n$ & 0 & $m$ \\
\hline
\end{tabular}

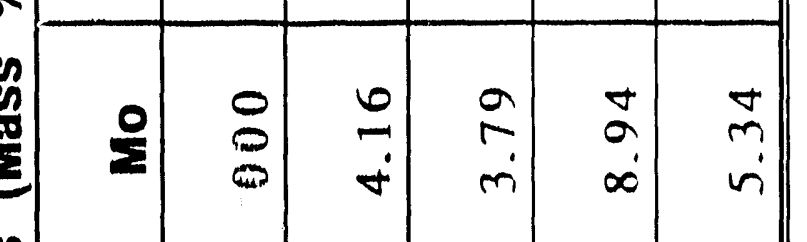

$\sqrt{N}$

ণ

늠

용

를

$m \pi$

놈 콬

즐 즘

当

营 苟

05

$\circ$

点

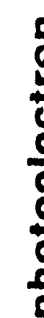

$\frac{5}{0}$

8

ते

$\frac{x}{x}<$

ง

요

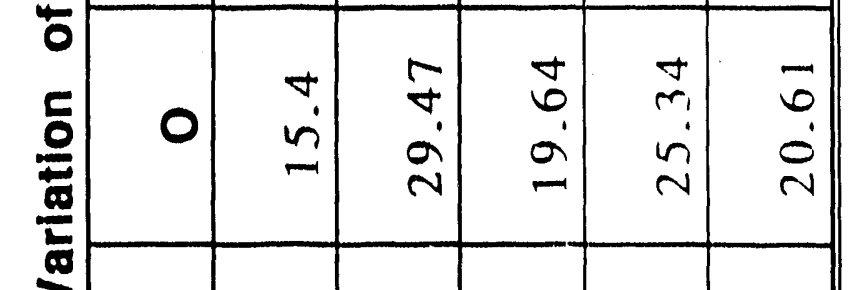

$\Xi$

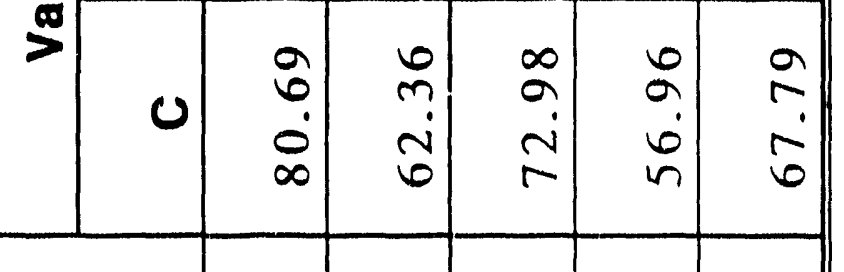

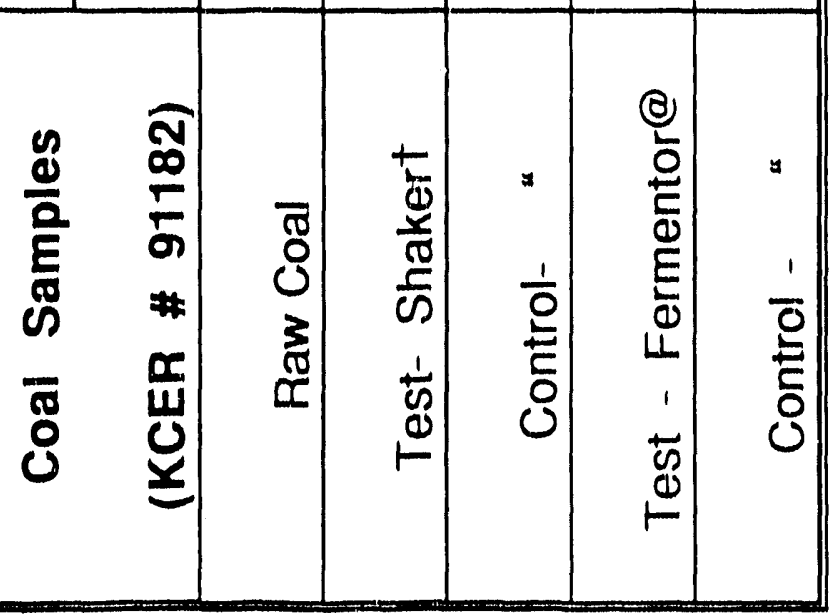

$\frac{0}{2}$ 造

总热

ปี

롤 恶㤩焉

文U记贻

$1 \frac{1}{0}$

造 


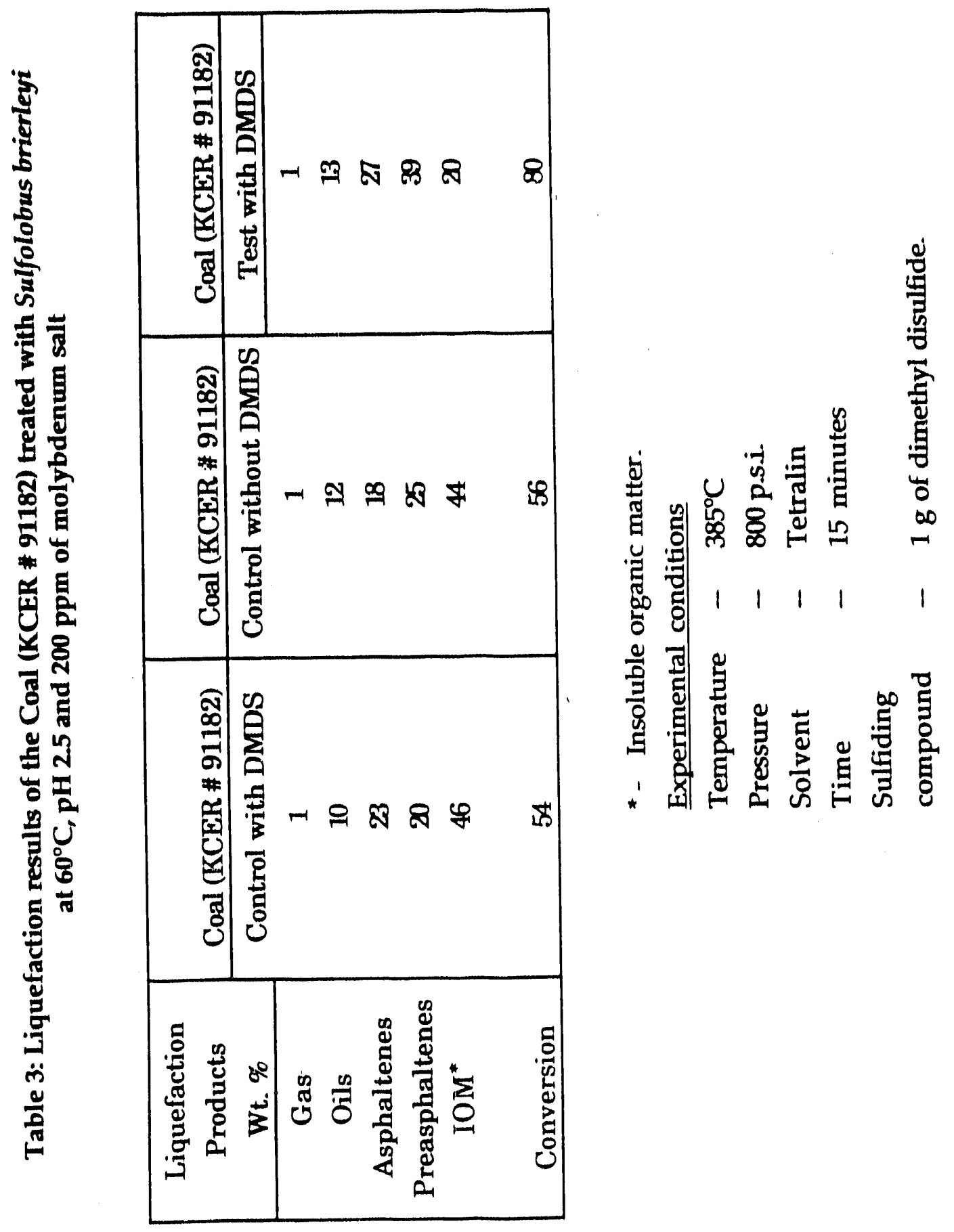


stonpodd Uolfobtenbli

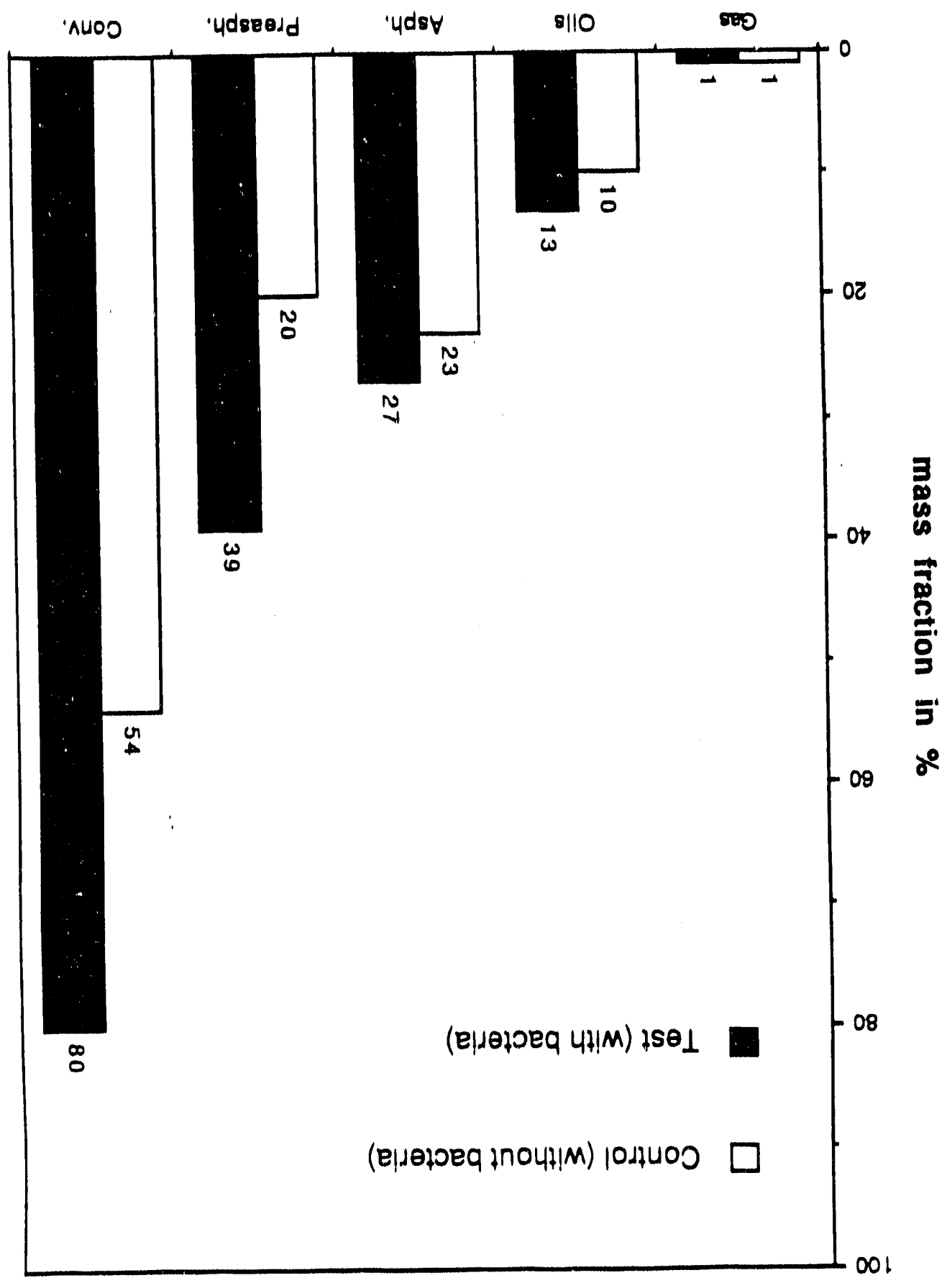

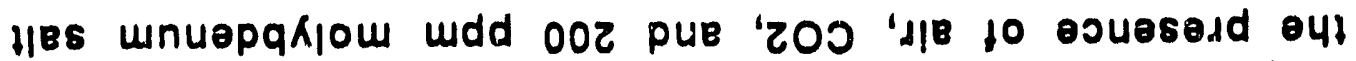

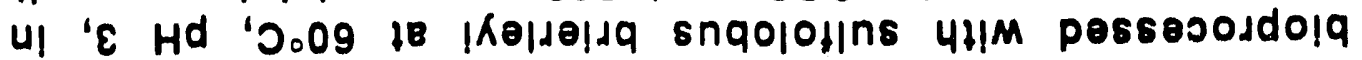

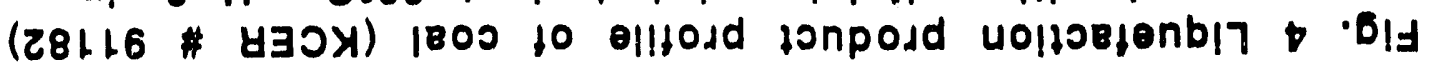


Fig. 5 Hydrogen uptake in presence of Desulfovibrio des!'lfuricans by a - naphthyl hexane

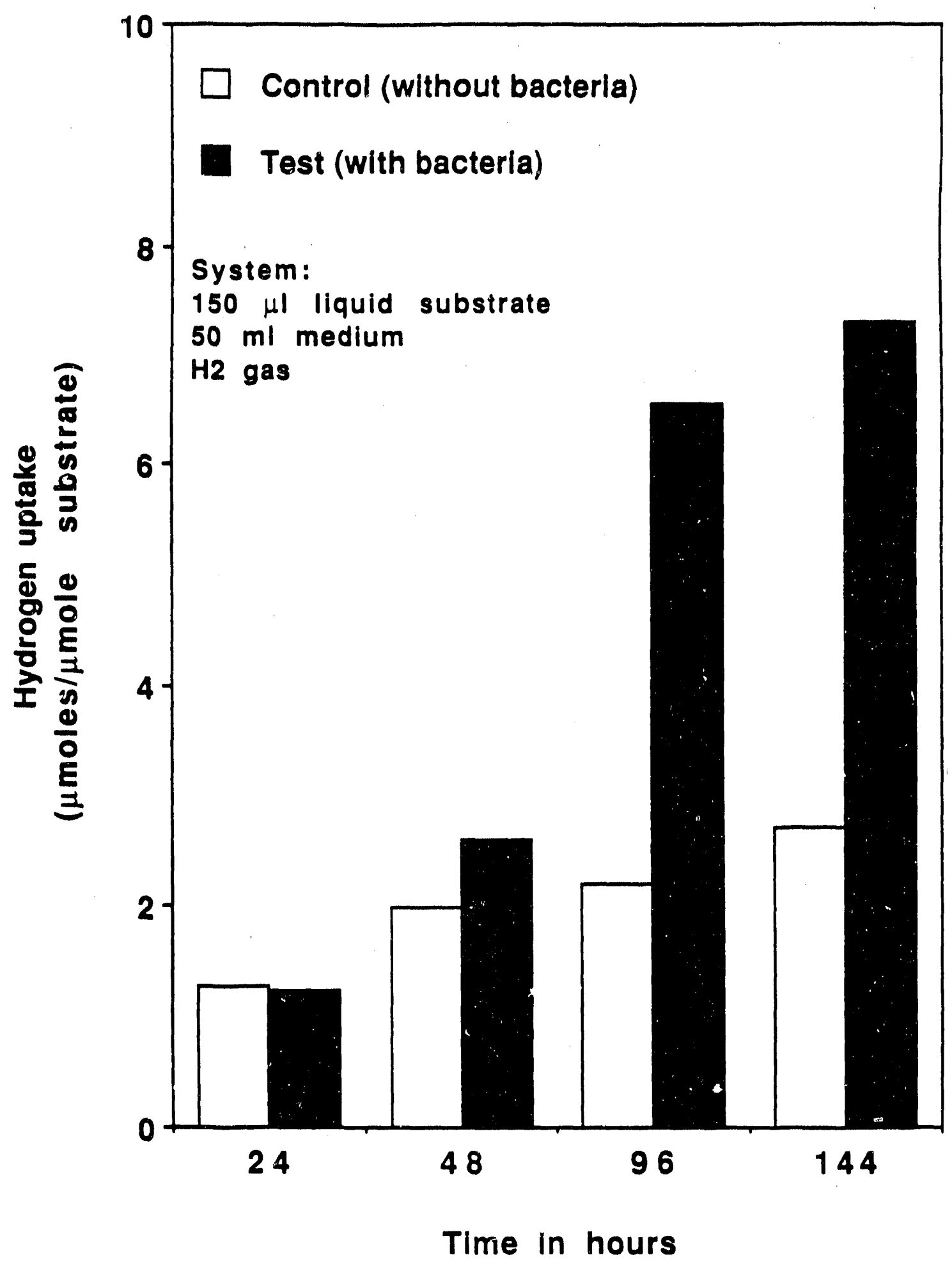


Fig. 6 Hydrogen uptake in presence of $D$. desulfuricans by 1,2-dlquinolyl ethane subjected to autoclaving condition

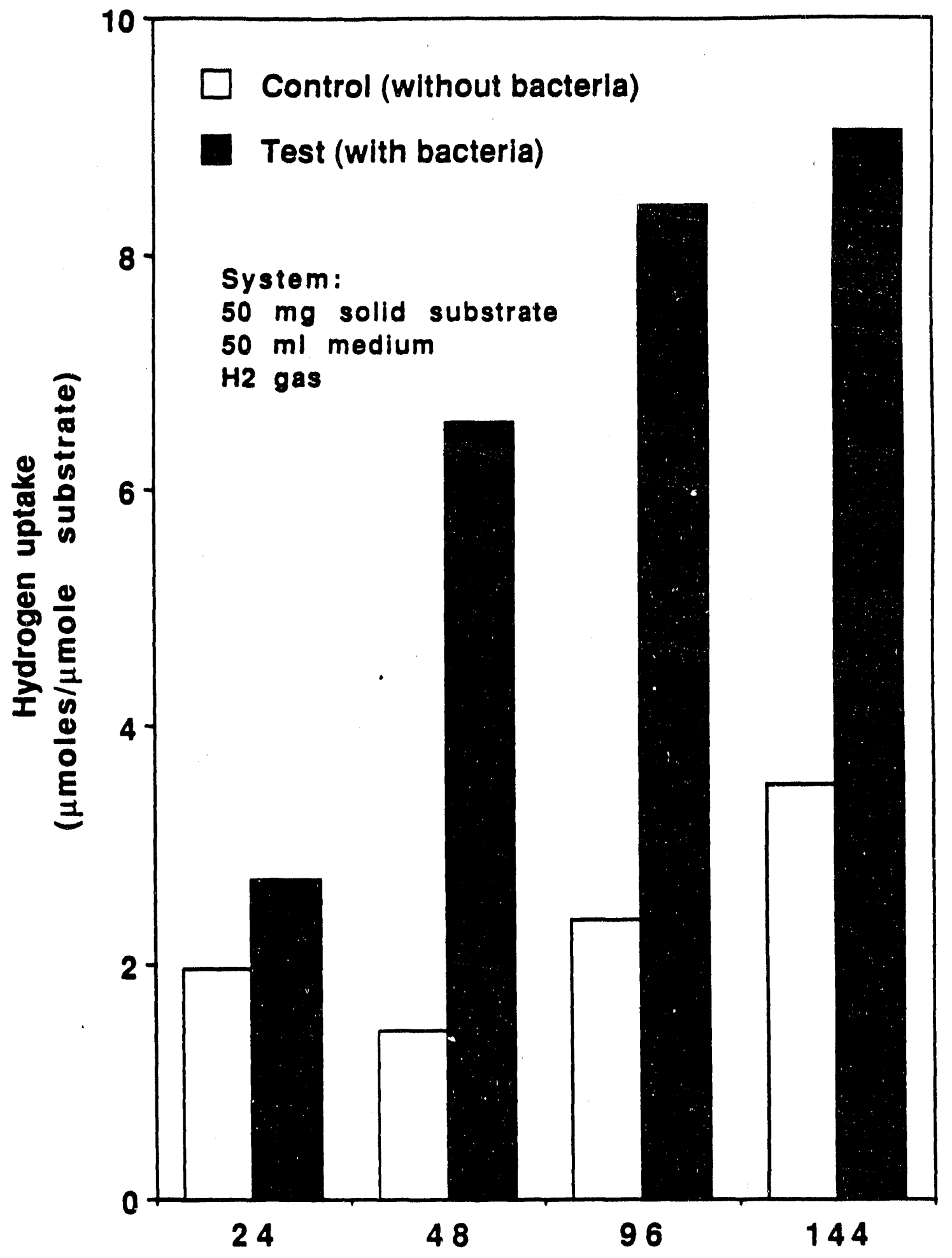

Time in hours 
Fig. 7 Hydrogen uptake in presence of $D$. desulfurlcans by 1,2-diquinolyl ethane dissolved in ethanol

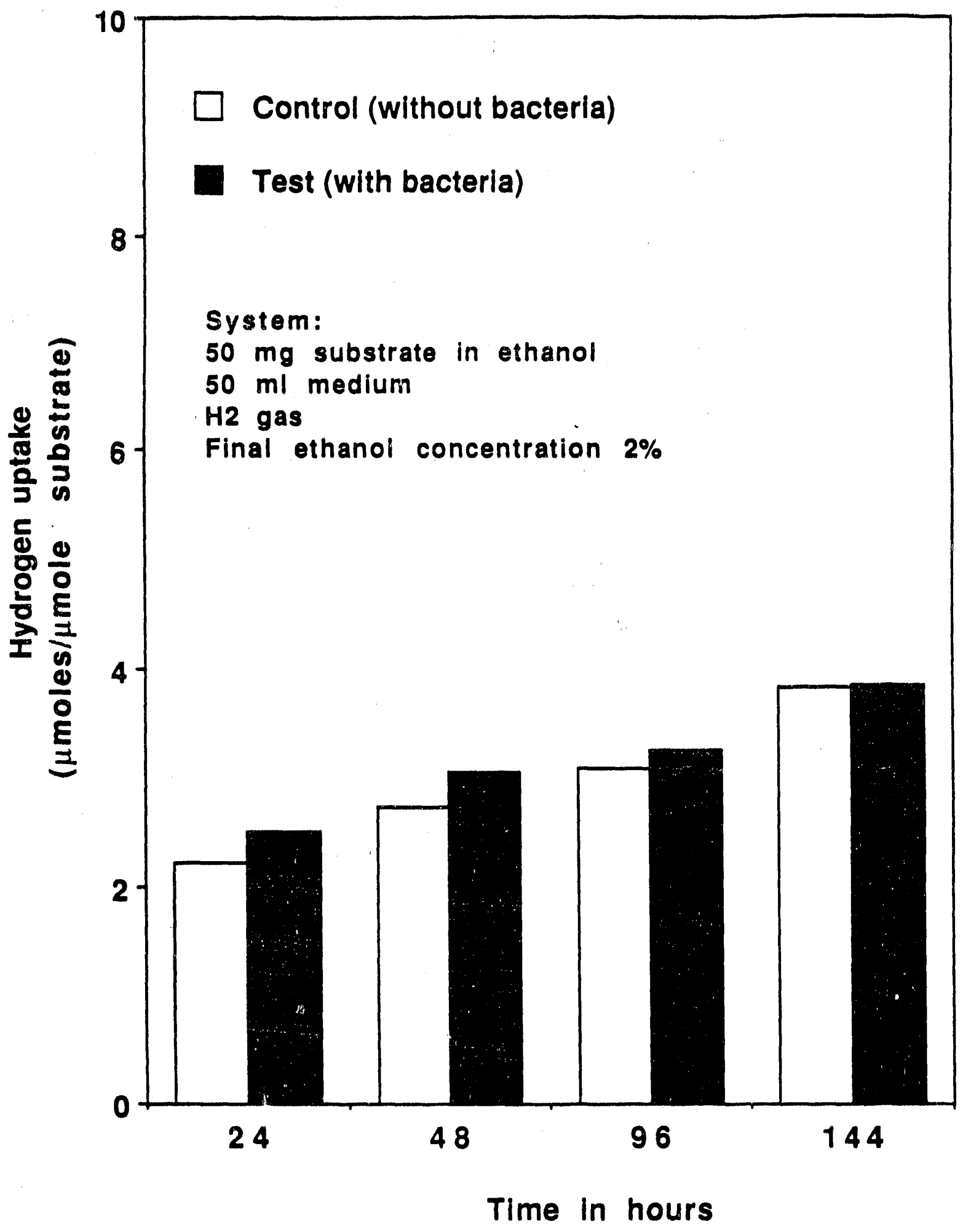


Fig.8: GC.MS analysis of diphenyl methane extracted with chloroform from aqueous medium of test treated with Desulfovibrio desulfuricans under hvdrogen atmosphere
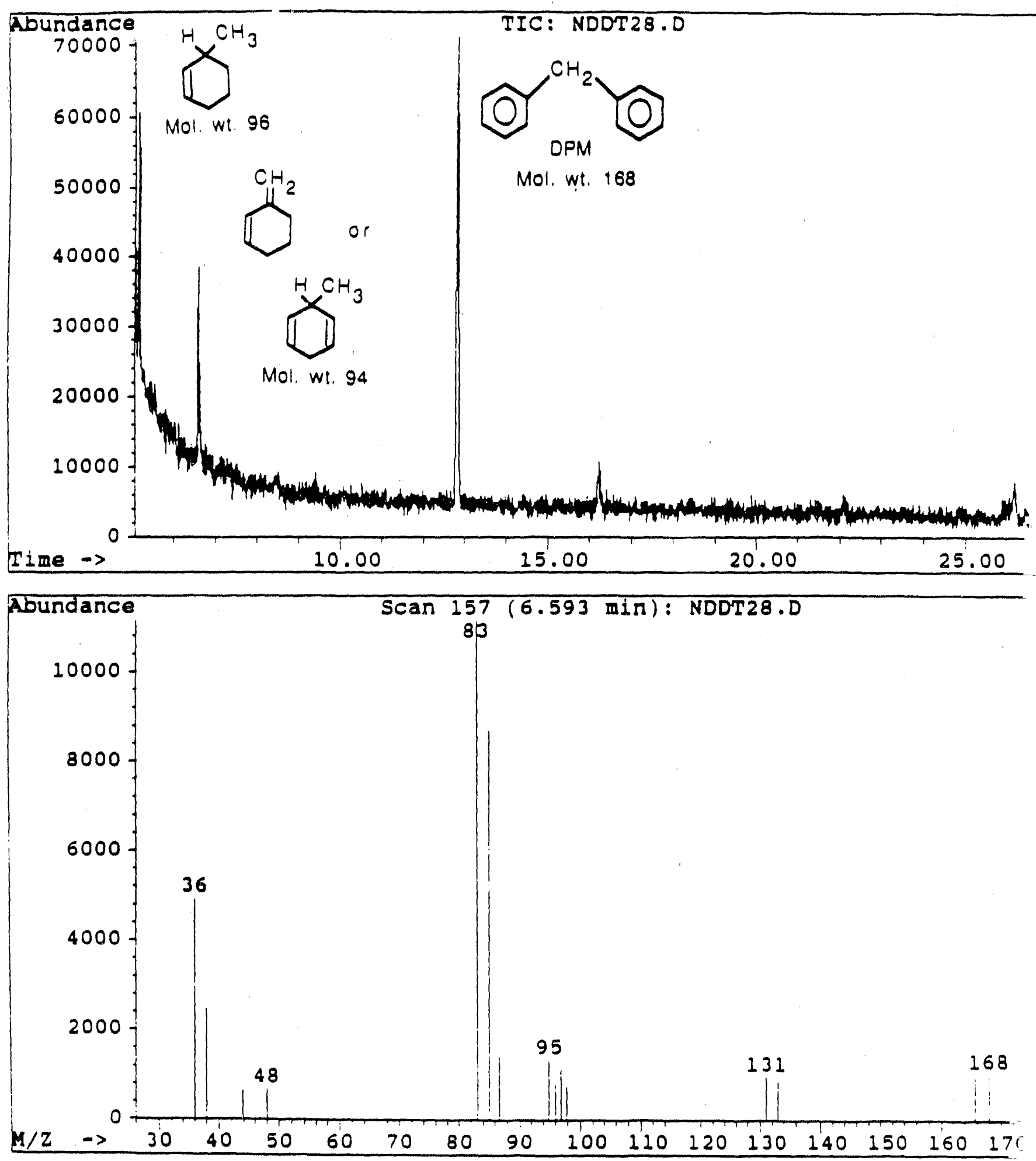
Fig.9: GC.MS analysis of diphenyl methane extracted with chloroform from aqueous medium of test treated with Sulfolobus brierleyi under hydrogen atmosphere

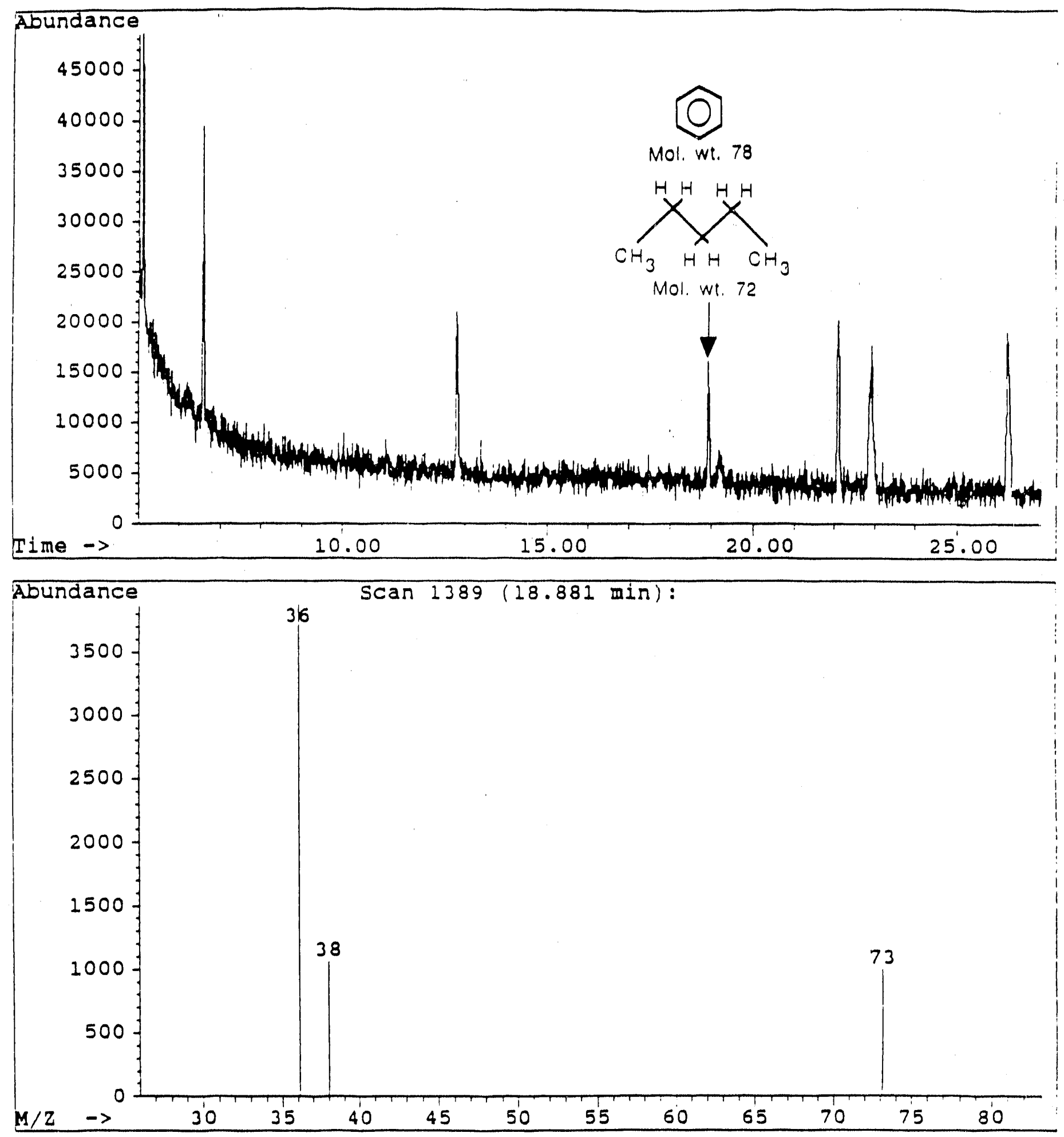


Fig.10: GC-MS analysis of diphenyl methane extracted with chloroform from aqueous medium of test treated with Sulfolobus brierleyi under hydrogen atmosphere

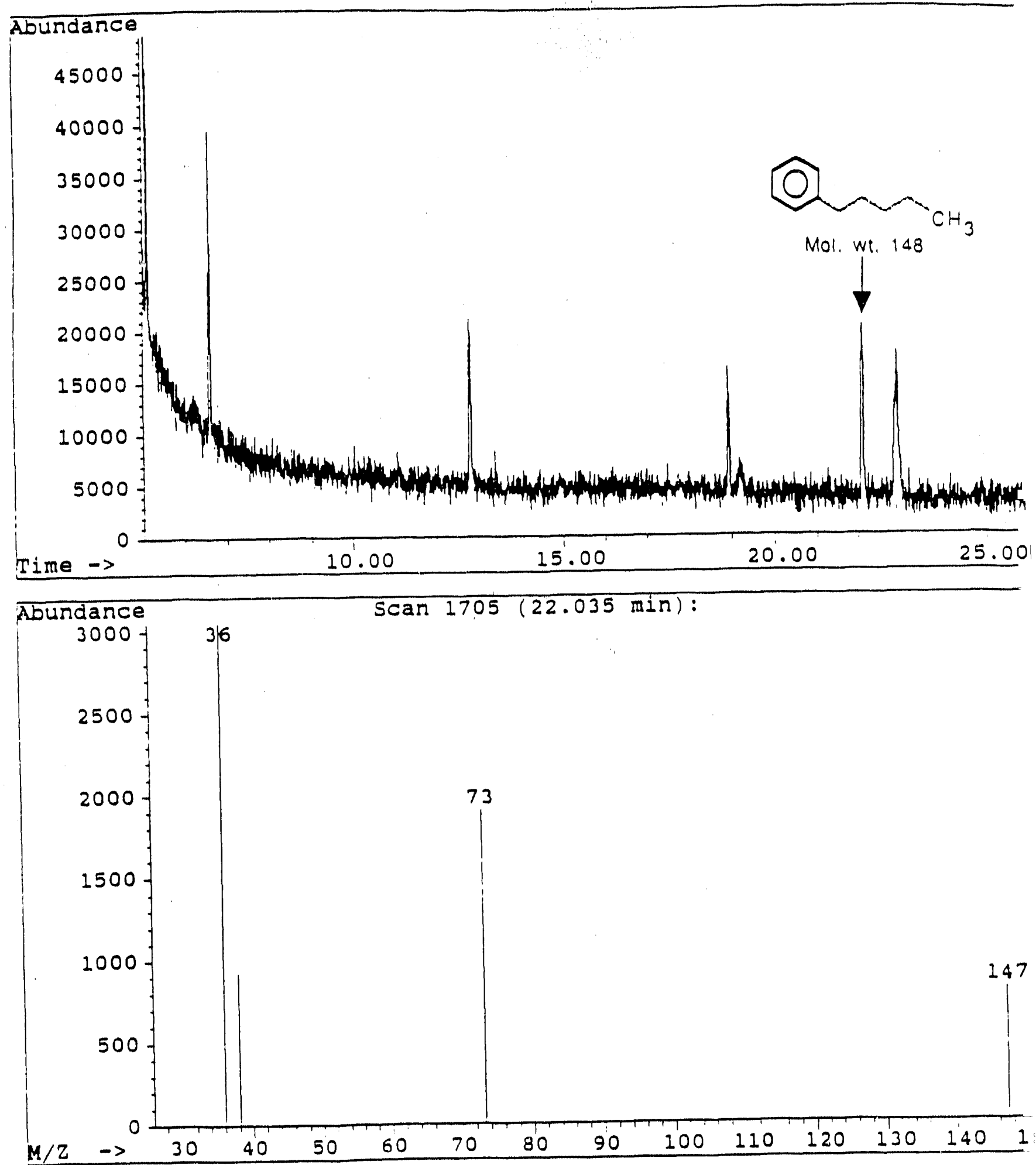


Fig.11: UV absorption spectra of the aqueous medium treated with Desulfovibrio desulfuricans in presence of coal (KCER \# 4677) and asphaltene for 144 hours.

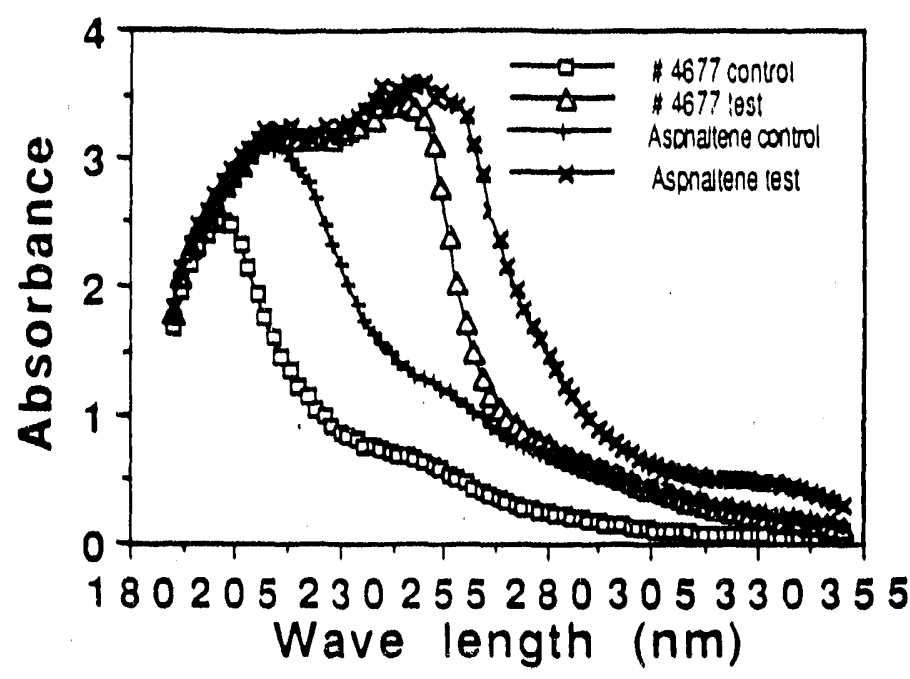

UV absorption spectra of the aqueous medium treated with Sulfolobus brierleyi in presence of coal (KCER \# 91182) and asphaltene for 144 hours.

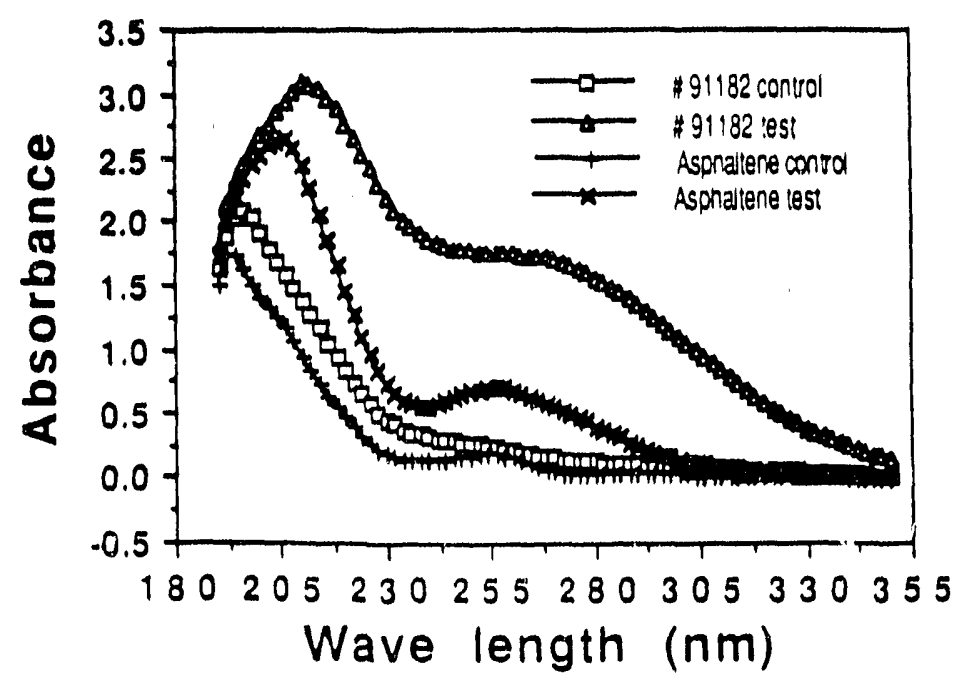




\title{
Project III.2 Distinguishing Chemical Reaction Mechanisms in Coal Liquefaction
}

\author{
John H. Penn \\ Department of Chemistry \\ West Virginia University
}

During the past contract period, additional model compounds have been synthesized which will allow for insight into the mechanisms of coal liquefaction using different catalysts. In addition, preliminary results relating to the solution phase cleavage of the 4-(1-naphthylmethyl)bibenzyl radical cation and other polyaromatic compounds have been obtained.

Understanding how a catalyst works is essential for catalyst development. Recently, Farcasiu, et al. ${ }^{1}$ have proposed that compounds containing both naphthyl and phenyl groups offer a number of advantages (e.g., high molecular weight, several different types of functional groups, and identifiable products which will be characteristic of a given reaction pathway) as models of chemical reactivity in coal. Representative of this . set of compounds is 4-(2- $\alpha$-naphthylethyl)diphenylmethane (1b, Figure 1$)$ which can undergo bond cleavage at any of the bonds labeled as A-E. Based upon the observed cleavage of Bond A for this compound under a variety of catalyst conditions, ${ }^{1}$ Farcasiu proposed that bond cleavage was occurring through radical ion chemistry (i.e., an electron transfer initiated reaction mechanism). This conclusion has been supported by theoretical studies $^{2}$ which predict that Bond $\mathrm{A}$ of this compound will be the preferred bond cleavage product of the radical cation. In contrast to this proposal is a broad spectrum of experimental data for bibenzylic systems ${ }^{3,4,5}$ which indicate that Bond $B$ is the preferred bond for cleavage of the radical cation in solution studies.

This apparent contradiction signifies that the mechanism of the catalyst action is incompletely understood. Our approach towards understanding the mechanistic dilemma of catalyst-promoted reactivity is to unambiguously establish the reaction pathway of radical cations (i.e., those species resulting from electron transfer pathways) in solution 
where a variety of techniques are avallable to generate radical cations. In addition, a number of new model compounds to firmly establish the location within the model compound from which the electron is transferred will be synthesized. The new set of target model compounds is shown in Figure 2.

\section{ACCOMPLISHMENTS}

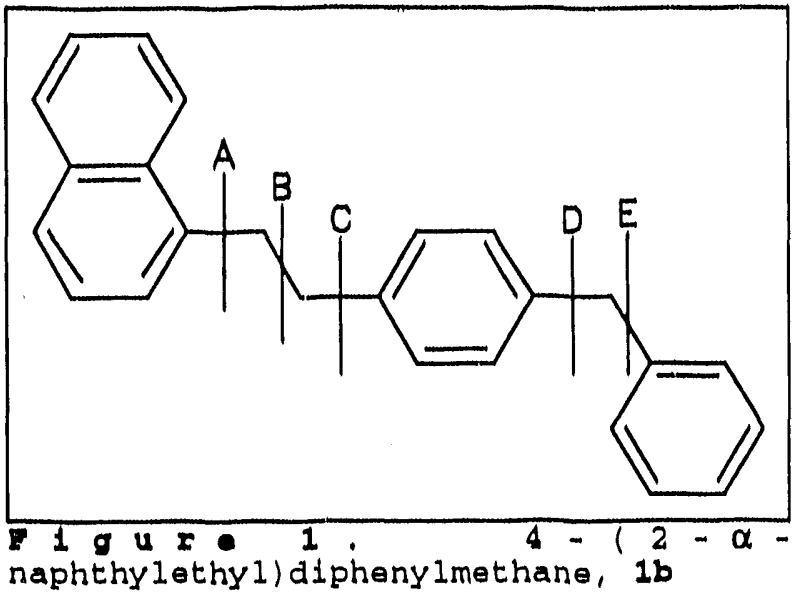

We report here that compounds $1 \mathbf{a}, 1 \mathbf{b}, \mathbf{2 a}$, and $3 \mathbf{a}$ of Figure 2 have been synthesized. Complete details of their synthesis is included in the experimental section. Items of particular note in the synthesis of these compounds include the fact that the Dowd synthesis ${ }^{1}$ was ineffective in our hands. To get reasonable yields of $\mathbf{1 a}$ and 1b, we resorted to the ferric chloride catalyzed reaction of appropriate alkyl chlorides to the appropriate diaryl compounds. Of additional note is the fact that attempted synthesis of compounds $\mathbf{2 b}$ and $\mathbf{3 b}$

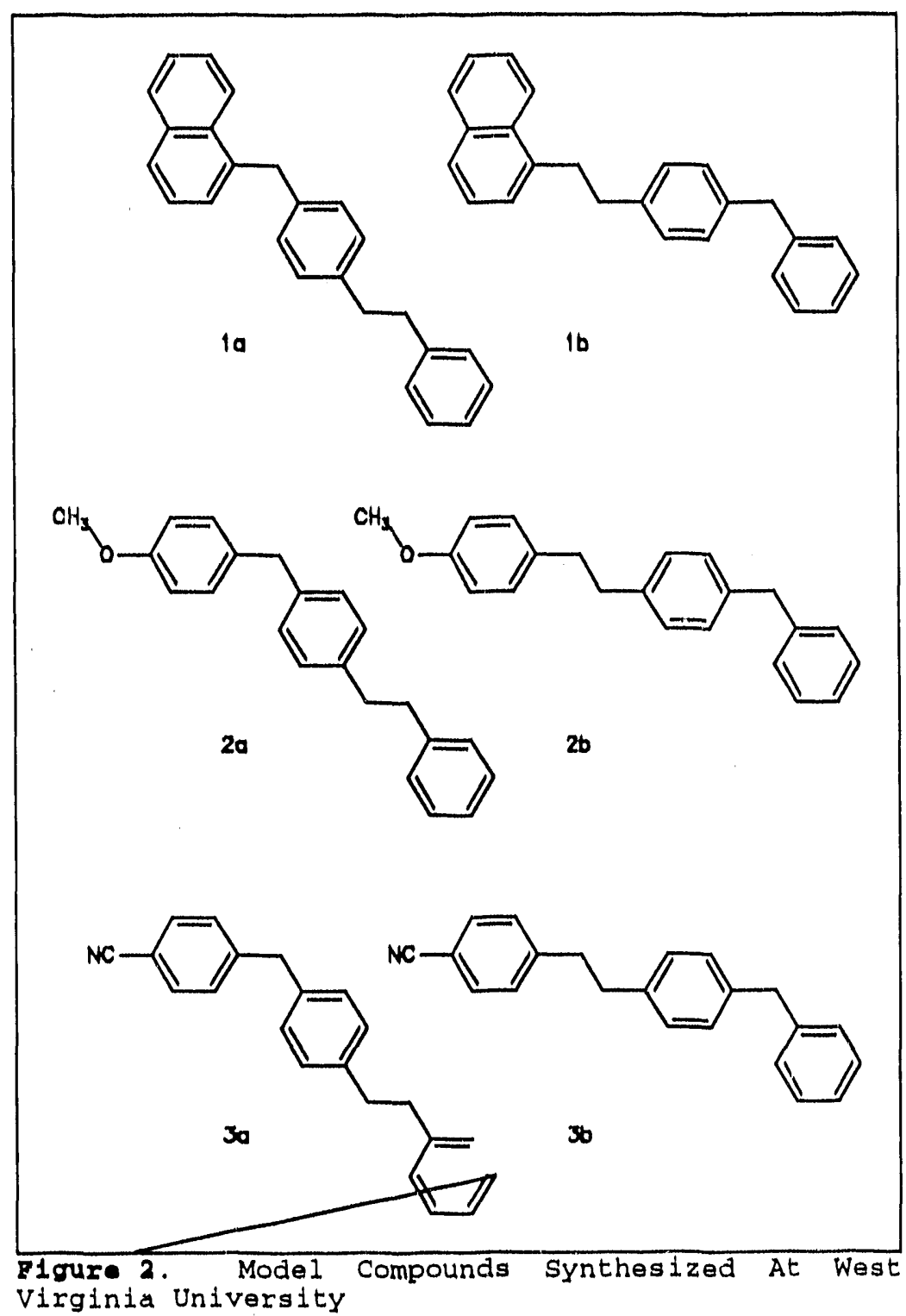


proved more difficult than expected. The synthesis of these compounds requires electrophilic aromatic substitution in unorthodox directions. Further progress on this problem is anticipated in the upcoming months of this contract.

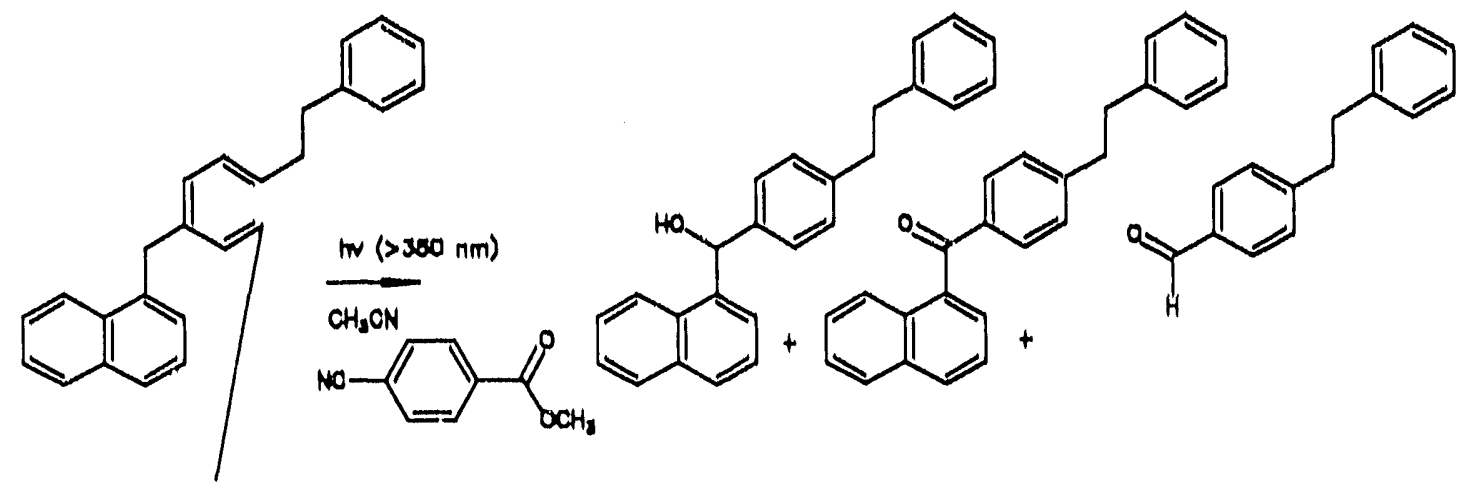

In addition to the synthesis of these model compounds, preliminary results related to the independent generation of the radical cations of $1 \mathrm{a}$ and $1 \mathrm{~b}$ have been performed. These experiments utilize the irradiation of methyl 4-cyanobenzoate in the presence of the appropriate electron donors in $\mathrm{CH}_{3} \mathrm{CN}$ solution. The excited state of methyl 4cyanobenzoate undergoes a one electron reduction to yield the radical cation of $\mathbf{1 a}$ or $\mathbf{1 b}$. Disappearance of both $1 \mathrm{a}$ and $\mathbf{1 b}$ are observed in these irradiations, indicating that the radical cations are reactive. The identifiable products at this point in time are shown in eq (1). Based on the observation of reactivity at the carbon between the naphthyl group and the bibenzyl group and also based on the observation of bibenzyl aldehyde, these results

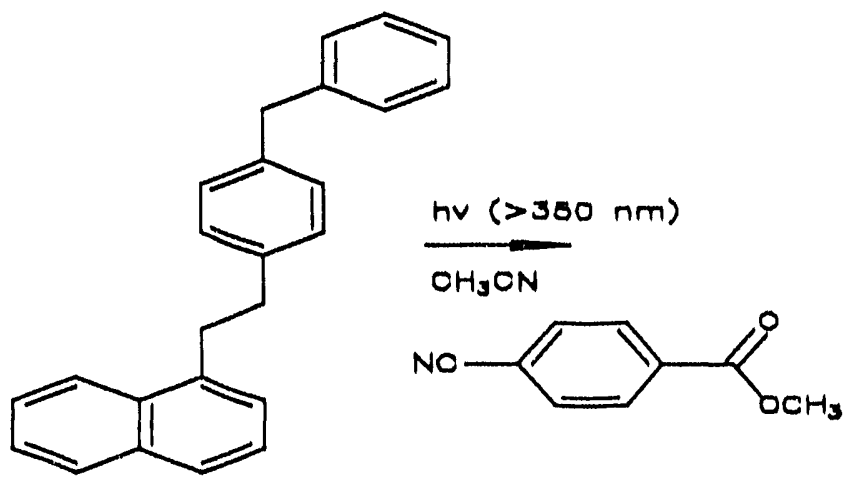<smiles>CCOc1ccc2cccc(C=O)c2c1</smiles> 
seem to indicate that bond $A$ or bond $B$ is broken in compound 1a. At the ourrent time, quantitative data are unavallable for this reaction. Reaction of $\mathbf{1 b}$ under the same conditions results in Bond B cleavage (Note eq (2)). This preliminary conclusion is based upon the observation of naphthaldehyde in the product mixture. This preliminary result supports the idea that solution radical cations behave as expected in solution, giving Bond B cleavage. Further quantitative measurements and verification of the entire product mixture are currently in progress to verify the results presented here.

\section{EXPERIMENTAL SECTION}

4-(1-naphthylmethyl)bibenzyl (1a): Ferric chloride $(57 \mathrm{mg}, 0.35 \mathrm{mmol})$ was transferred into a flame dried $50 \mathrm{~mL}$ round-bottomed flask in a glove box followed by addition of dry $\mathrm{CS}_{2}(8 \mathrm{~mL})$. The resulting mixture was stirred for 10 minutes and cooled to $0^{\circ} \mathrm{C}$. Solid bibenzyl was added and stirred for $10 \mathrm{~min}$. A solution of 1 -(chloromethyl)naphthalene $(706.6 \mathrm{mg}, 4.0 \mathrm{mmol})$ in dry $\mathrm{CS}_{2}(5 \mathrm{~mL})$ was added dropwise into the reaction flask over 5 hours while maintaining a small dry nitrogen stream through the flask to bring out excess $\mathrm{HCl}$ formed during the reaction. The resultant mixture was stirred for 5 hours at room temperature. The reaction mixture was poured into ice water $(20 \mathrm{~mL})$ containing concentrated $\mathrm{HCl}(3 \mathrm{~mL})$, diluted with $\mathrm{CH}_{2} \mathrm{Cl}_{2}$ $(20 \mathrm{~mL})$, shaken thoroughly, and transferred into a separatory funnel. The organic layer was separated and the aqueous layer was extracted with $\mathrm{CH}_{2} \mathrm{Cl}_{2}(2 \times 5 \mathrm{~mL})$. The combined organic extracts were decolored with activated charcoal, filtered and evaporated in vacuo to give a clear oil (1048 $\mathrm{mg})$. Silica gel column chromatography eluted with 1:4 $\mathrm{CH}_{2} \mathrm{Cl}_{2}$ /hexane was used to recover bibenzyl (802mg, 80.1\%) and the desired naphthylmethylbibenzyl $(127.9 \mathrm{mg}, 9.9 \%)^{5}$ as a colorless solid, m.p. $64.0-64.5^{\circ} \mathrm{C}$ (hexane).

\footnotetext{
When calcium chloride was used as a dispersant and sodium phosphate as a base to neutralize the hydrogen chloride formed in reaction, the yield (12.18) may be raised a little at the expense of a longer reaction time.
} 
4-Chloromethyldiphenylmethane (2): To a mixture of concentrated hydrochloric acid $(45 \mathrm{~mL}), 85 \%$ phosphoric acid $(24 \mathrm{~mL})$ and glacial acetic acid $(30 \mathrm{~mL})$ was added paraformaldehyde $(13.5 \mathrm{~g}, 0.45 \mathrm{~mol})$. Hydrogen chloride was passed into the resulting mixture in a fine stream while the flask was heated. At $80^{\circ} \mathrm{C}$, diphenylmethane $(50.47 \mathrm{~g}$, $0.3 \mathrm{~mol}$ ) was added in une portion. The reaction mixture was kept at $110^{\circ} \mathrm{C}$ with vigorous stirring for 96 hours. After the mixture was cooled to room temperature, $60 \mathrm{~mL}$ of hexane was added and shaken thoroughly. The organic layer was separated, washed $3 \mathrm{X}$ with water, dried with anhydrous $\mathrm{Na}_{2} \mathrm{SO}_{4}$, decolored with activated charcoal, and passed through a $2 \mathrm{~cm}$ silica gel column to give a colorless solution. Evaporation of solvent in vacuo left a slightly green oil. Purification by silica gel chromatography using hexanes gave a colorless oil $(31.5 \mathrm{~g}$, yield $48.2 \%)$.

4-[2-(1-naphthyl)ethyl]diphenylmethane (1b): Magnesium turnings $(0.261 \mathrm{~g}$, $10.75 \mathrm{mmol}$ ) were placed in a flame dried flask and covered with $5 \mathrm{~mL}$ of freshly distilled ether (from $\mathrm{LiAlH}_{4}$ ), followed by addition of a catalytic amount of lodine for initiating the reaction. 1-Chloromethylnaphthalene $(1.89 \mathrm{~g}, 10.7 \mathrm{mmol})$ in $10 \mathrm{~mL}$ of ether was added dropwise to maintain a gentle reflux. After the addition was complete, the reaction suspension was refluxed for an additional $30 \mathrm{~min} .20 \mathrm{~mL}$ of freshly distilled dry THF (from $\mathrm{LiAlH}_{4}$ ) was added and the ether was removed by distillation. The resultant yellow solution was cooled to ca. $0--5^{\circ} \mathrm{C}$. 4-chloromethyl-diphenylmethane $(2.284 \mathrm{~g}$, $10.5496 \mathrm{mmol})$ in $5 \mathrm{~mL}$ of THF was added in one portion and a $\mathrm{Li}_{2} \mathrm{CuCl}_{4}$ solution $(0.1 \mathrm{M}$ in THF, $0.4 \mathrm{~mL}$ ) was immediately added and vigorously stirred for 20 minutes. The resultant solution was poured into $60 \mathrm{~mL}$ of petroleum ether and washed with saturated sodium bicarbonate $(50 \mathrm{~mL})$, water $(50 \mathrm{~mL})$ and dried $\left(\mathrm{Na}_{2} \mathrm{SO}_{4}\right)$. Evaporation of the solvent in vacuo yielded a yellow oil which was purified by chromatography (silica gel, hexanes) to yield $0.8339 \mathrm{~g}(24.7 \%)$ of the desired 3.

4-Chloromethylbibenzyl (4): To a mixture of concentrated hydruchloric acid $(30 \mathrm{~mL}), 85 \%$ phosphuric acid $(16 \mathrm{~mL})$ and glacial acetic acid $(20 \mathrm{~mL})$ was added paraformaldehyde $(9.0 \mathrm{~g}, 0.3 \mathrm{~mol})$. Hydrogen chloride was passed through the mixture in a fine stream while the flask was heated. At $80^{\circ} \mathrm{C}$, bibenzyl $(36.4 \mathrm{~g}, 0.2 \mathrm{~mol})$ was added in one portion. The reaction mixture was kept at $95^{\circ} \mathrm{C}$ with vigorous stirring for 72 hours. 
The mixture was cooled to room temperature, $50 \mathrm{~mL}$ of hexane was added and shakened thoroughly. The organic layer was separated, washed $3 \mathrm{X}$ with water, dried $\left(\mathrm{Na}_{2} \mathrm{SO}_{4}\right)$, and passed through a $2 \mathrm{~cm}$ silica gel column to give a colorless solution. Removal of solvent in vacuo left an oil which was separated by vacumm distillation $\left(155-170^{\circ} \mathrm{C} / 0.6-\right.$ $0.8 \mathrm{~mm} \mathrm{Hg}$ ) to yield the expected product ( $15.7287 \mathrm{~g}$, yield $34 \%)$.

4-(4'-Methoxybenzyl)bibenzyl (2a): Ferric chloride (120 mg) and calcium chloride $(600 \mathrm{mg})$ were transferred into a flame dried $100 \mathrm{~mL}$ round-bottomed flask in a glove box to which was added $30 \mathrm{~mL}$ of dry $\mathrm{CS}_{2}$. The resulting mixture was stirred for $10 \mathrm{~min}$ at room temperature and then anisole $(10.8 \mathrm{~g}, 100 \mathrm{mmol})$ was added in one portion. After stirring for an additional $10 \mathrm{~min}$, a solution of 4-chloromethylbibenzyl $(4.61 \mathrm{~g}, 20 \mathrm{mmol})$ in dry $\mathrm{CS}_{2}(20 \mathrm{~mL})$ was added dropwise over $30 \mathrm{~min}$ while a small stream of dry nitrogen was passed through the flask to eliminate excess hydrogen chloride. The mixture was stirred for an additional 1 hour at room temperature. The reaction mixture was filtered and concentrated in vacuo to give a yellow oil which was purified by column chromatography (silica gel, hexane $/ \mathrm{CH}_{2} \mathrm{Cl}_{2}=75 / 25$ ) to yield 3-(4'methoxybenzyl)-bibenzyl $(1.5044 \mathrm{~g})$ and 4-(4'-methoxybenzyl)bibenzyl $(1.7902 \mathrm{~g})$. Total yield: $54.5 \%$.

4-(4'-hydroxylbenzyl)bibenzyl (8): Ferric chloride $(120 \mathrm{mg})$, calcium chloride $(600 \mathrm{mg})$, and dry $\mathrm{CS}_{2}(30 \mathrm{~mL})$ were transferred into a flame dried $100 \mathrm{~mL}$ roundbottomed flask in a glove box. The resulting mixture was stirred for $10 \mathrm{~min}$ at room temperature and phenol $(9.4 \mathrm{~g}, 100 \mathrm{mmol})$ was added as a solid. After stirring for an additional $10 \mathrm{~min}$, a solution of 4-(chloromethyl)bibenzyl $(4.61 \mathrm{~g}, 20 \mathrm{mmol})$ in dry $\mathrm{CS}_{2}$ $(20 \mathrm{~mL})$ was added dropwise over a period of 0.5 hours while a small dry nitrogen stream was passed through the flask to remove excess hydrogen chloride formed during the reaction. The mixture was stirred for an additional 1 hour at room temperature. The reaction mixture was filtered and concentrated in vacuo. $30 \mathrm{~mL}$ of hexane was added, washed with water $5 \mathrm{X}$ to remove the excess phenol. The crude product was purified by column chromatography (silica gel, hexane $/ \mathrm{CH}_{2} \mathrm{Cl}_{2} /$ ethanol $=9.5 / 0.35 / 0.15$ ) to yield $0.9145 \mathrm{~g}(15.9 \%)$ of colorless crystal.

4-(4'-Cyanobenzyl)bibenzyl (3a): Ferric chloride $(90 \mathrm{mg})$, calcium chloride 
$(600 \mathrm{mg})$, and dry $\mathrm{CS}_{2}(30 \mathrm{~mL})$ were transferred into a flame dried $100 \mathrm{~mL}$ roundbottomed flask in a glove box to which was added $\alpha$-bromo-toluenitrile $(2.94 \mathrm{~g}, 15 \mathrm{mmol})$ and bibenzyl $(10.94 \mathrm{~g}, 60 \mathrm{mmol})$. The resulting mixture was heated and stirred at $110^{\circ} \mathrm{C}$ for 60 hours while a small dry nitrogen stream was passed through the flask to remove excess hydrogen chloride formed during the reaction. The reaction mixture was cooled to room temperature, extracted with hexanes, and concentrated in vacuo to yield a brown oil. The unreacted bibenzyl was removed by distillation under reduced pressure. The residual oil was chromatographed (silica gel, hexane/ethanol $=100 / 0.5$ ) to give a mixture of two products $(1.64 \mathrm{~g}$, yield $36.8 \%)$ of meta- and para-substituted products.

\section{REFERENCES}

1. a) Farcasiu, M.; Smith, C. Energy Fue1s, 1991, 5(1), 83-7. b) Farcasiu, M.; Smith, C. Prepr. Pap. - Am. Chem. Soc., Div. Fuel Chem., $1990,35(2), 404-13$.

2. a) Ades, Harriet F.; Companion, Audrey L.; Subbaswamy, K. R. J. Phys. Chem., 1991, 95(17), 6502-7. b) Ades, Harriet F.; Companion, Audrey L.; Subbaswamy, K. R. Prepr. Pap. - Am. Chem. Soc., Div. Fuel Chem., 1991, 36(1), 420-4. C) Ades, Harriet F.; Companion, Audrey L.; Subbaswamy, $K$. R. J. Phys. Chem., 1991, 95(6), 2226-31.

3. Penn, J.H.; Lin, Z.; Deng, D.-L. J. Am. Chem. Soc., 1991, 113, 1001.

4. Bacciochi, E.; Bartoli, D.; Rol, C.; Ruzziconi, R.; Sebastiani, B.V. J. Org. Chem., 1986, 51, 3587 .

5. a) Camaioni, D.M. J. Am. Chem. Soc., 1990, 112, 9475. b) Camaioni, D.M.; Franz, J.A. J. Org. Chem., 1984, 49, 1607. 


\title{
Project III.3 Better Hydrogen Transfer in Coal Liquefaction
}

\author{
A.R. Tarrer \\ Auburn University
}

\section{Introduction.}

During the past quarter, our efforts have been focused on three primary tasks: (1) design/construction of a continuous flow reactor system, (2) improving our mass transfer contactor design, and (3) disseminating results from earlier studies.

\section{Construction of the reactor system.}

We initially decided to do the construction in three stages. A schematic for the first stage system is shown in Figure 1. Much of the reactor's design is pattemed after the design of an eartier reactor system constructed and used to perform catalyst deactivation studies at Aubum $(1,2,3)$. The letdown systems, holding tanks, level controls, samplers, and gas-liquid separators are essentially the same design. The earlier unit is not suitable, however, for performing the studies intended here. Schematics of the earlier system with equipment specifications are given in Figures 2, 3, and 4 and in Table 1.

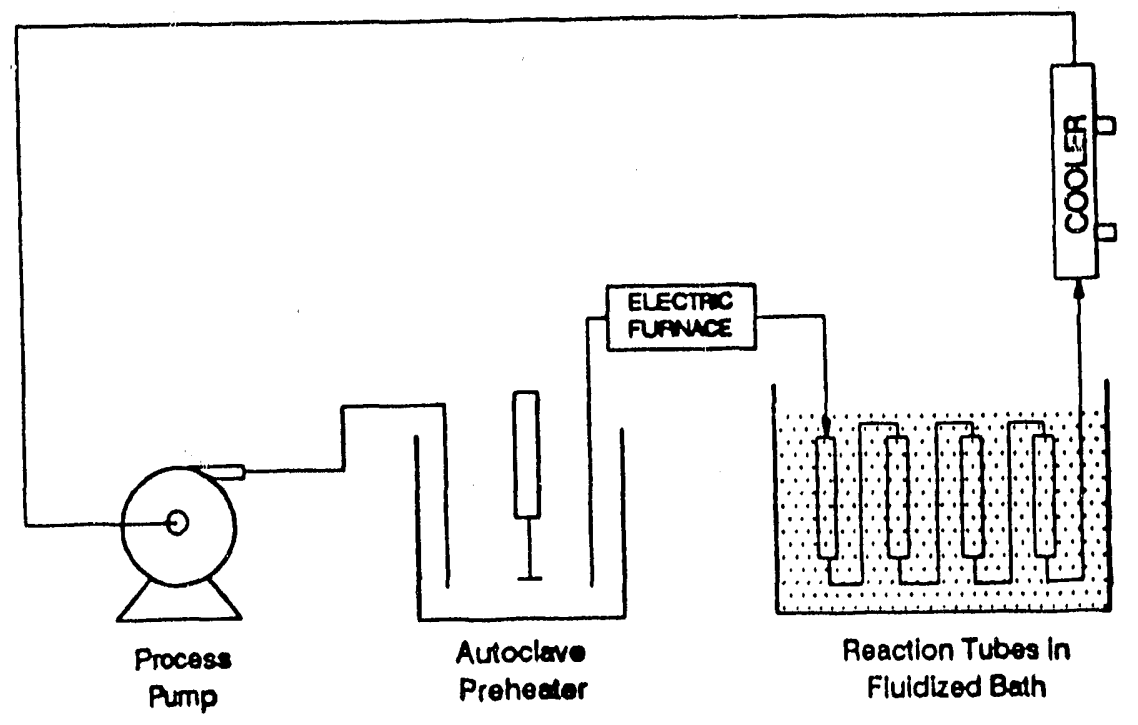

Figure 1: Schematic of Stage 1 Reactor System 

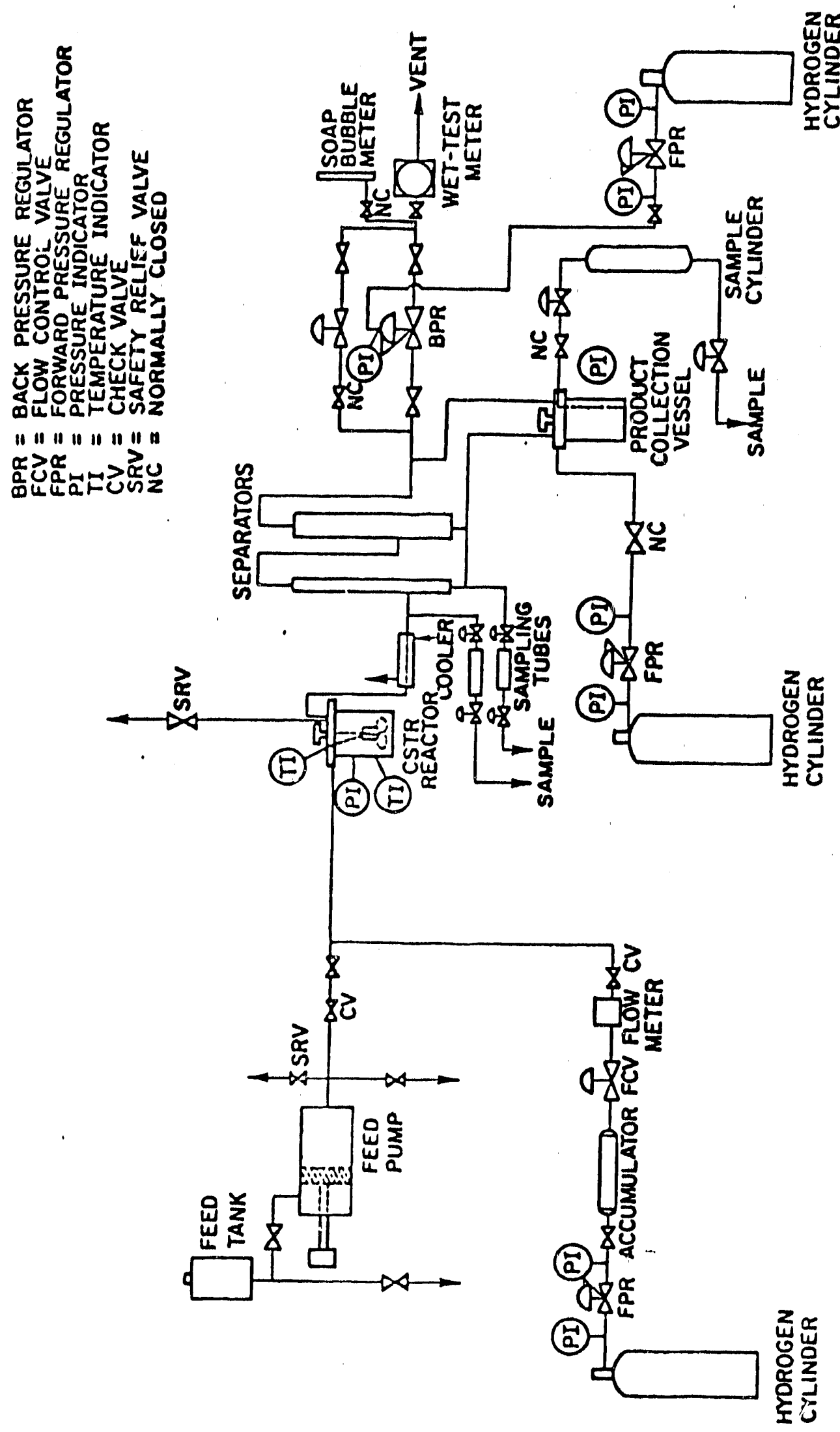

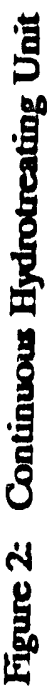




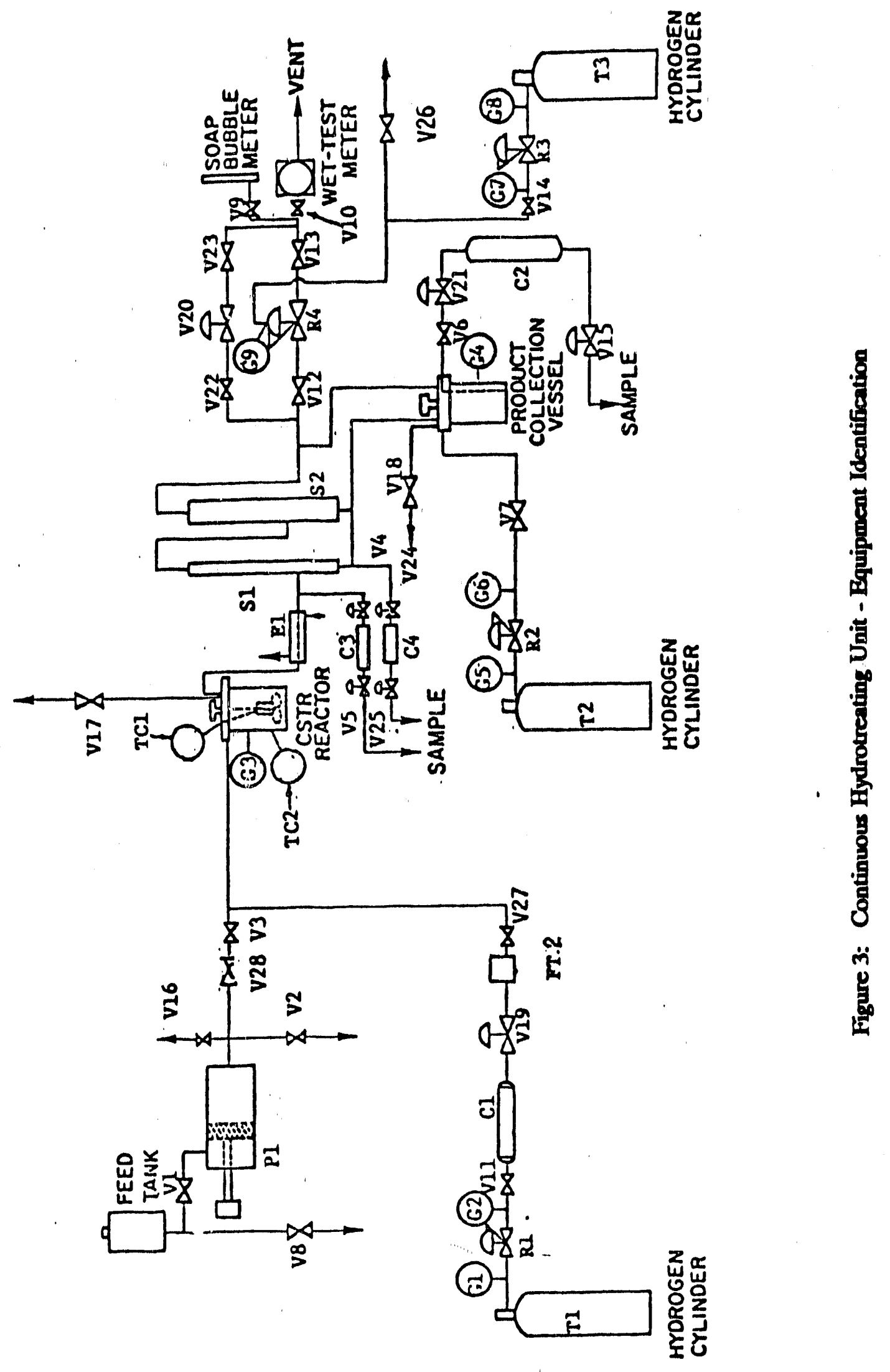




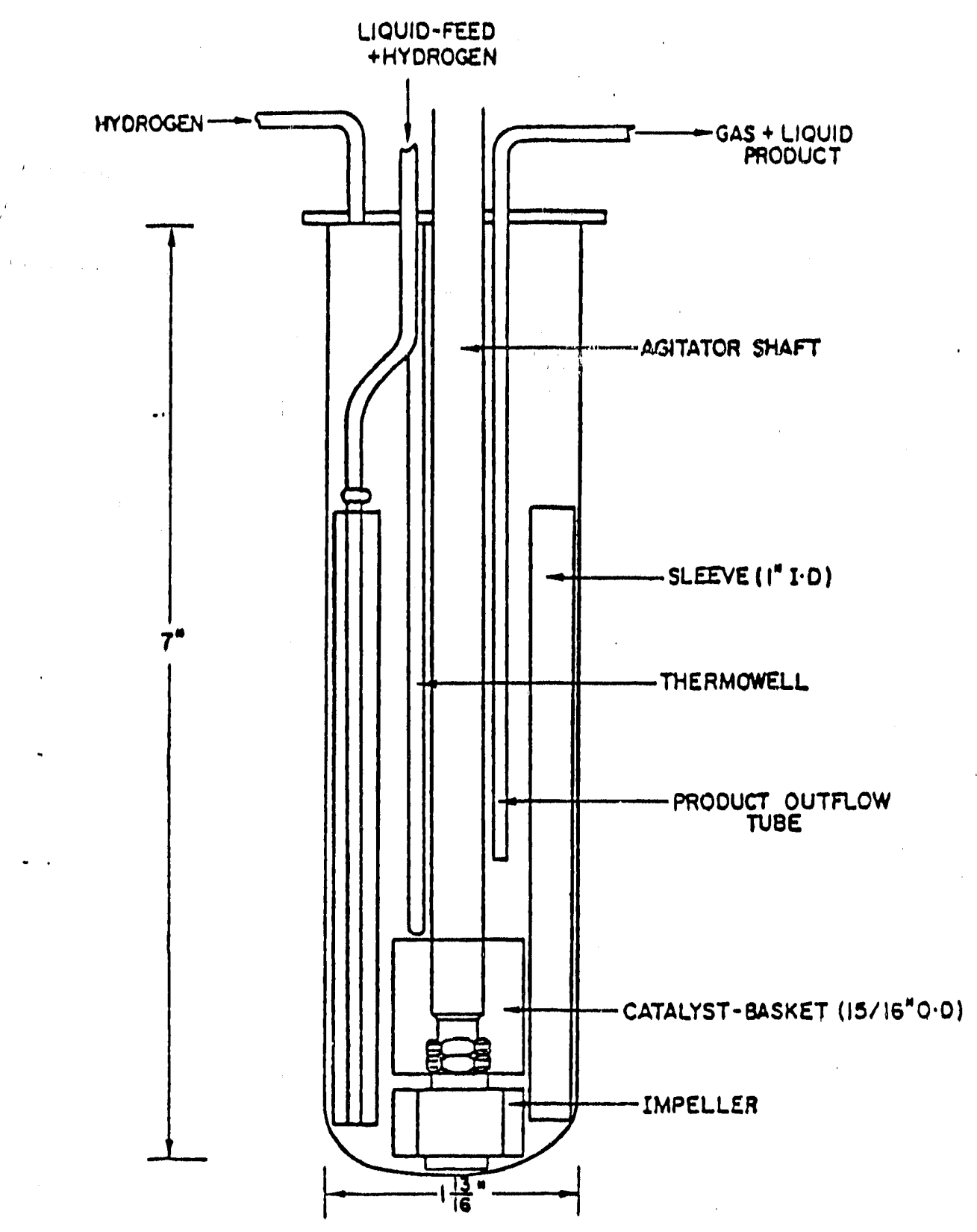

Figure 4: Spinning Basket Reactor 


\section{Table 1}

Equipment Specifications for the Continuous Hydrotreating Unit

\begin{tabular}{lll}
\hline & & \\
\hline Identi- & Use & Description \\
fication & & \\
\hline
\end{tabular}

Valves:

V1

V2

V3

$\mathrm{V} 4,5,6$

V7

V8

v9

V10

V11

V12,13

V14
Pump Syringe

Charge Valve

Pump Syringe

Drain Valve

Liquid Feed

Shut-Off Valve

Liquid

Sampling Valve

Hydrogen Shut-

Off Valve

Feed Tank Drain Valve

Wet-Test Meter

By-Pass Valve

Soap Bubble

Meter By-Pass

Valve

Hydrogen Shut-Off Valve

R4 By-Pass Valves

Hydrogen Shut-Off

Valve
$1 / 4^{n}$ Hoke ball vatue ( $711564 Y$ ), 0.25 in. orifice, $C_{v}=1.4$, $316 \mathrm{SS}, 6000 \mathrm{psi}$ pressure rating, $175^{\circ} \mathrm{C}$ maximum temperature.

$1 / 4^{\mathrm{n}}$ Nupro plug valve (4P4T), $0.093 \mathrm{in}$. orifice, $\mathrm{C}_{\mathrm{v}}=0.63$, $316 S 5,3000$ psi pressure rating, $200^{\circ} \mathrm{C}$ maximum temperature.

$1 / 4^{n}$ Whitey shut-off Valve (SS-6VF4), 0.25 in. orifice, $316 \mathrm{SS}, \mathrm{C}_{\mathrm{v}}=0.85,5000$ psi pressure rating, $450^{\circ} \mathrm{C}$ maximum temperature. 
V15

V16

V17

V18

V19

V20

V21

V22,23

V 24,25

V26

V27,28

Requlators:

R1
Collection

Vessel Drain

Valve

Pump Safety

Head

Reactor

Safety Head

Collection

Vessel Safety

Head

Hydrogen

Metering Valve

Product Gas

Flow Control

Valve

Throttling

Valve

V25 By-Pass

Valve

Liquid

Sampling

Valves

Pressure

Release Valves

Check Valves
$1 / 4^{\prime \prime}$ Whitey regulating valve (SS-6LRF4), 0.25 in. orifice, $316 \mathrm{SS}, \mathrm{C}=0.85,5000$ psi pressure rating, $450^{\circ} \mathrm{C}$ maximum temperature.

$1 / 4^{n}$ Autoclave Engineers' safety head, 3000 psi pressure rating for rupture disc, $480^{\circ} \mathrm{C}$ maximum temperature.

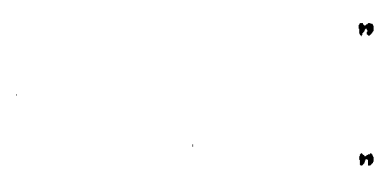

$1 / 4^{n}$ Hoke Milli-Mite forged metering valve (1335G4B), $0.047^{*}$ orifice, $\mathrm{Cv}=0.01,316 \mathrm{SS}, 5000$ psi pressure rating.

1/4" Autoclave Engineers regulating valve (10V4081), $0.125^{\prime \prime}$ orifice, $\mathrm{Cv}=0.86,11500$ psi pressure rating.

$1 / 4^{n}$ Nupro fine metering valve (SS-4SA), $0.031^{\text {" orifice, }}$ $\mathrm{Cv}=0.004^{n}, \mathrm{SS} 316,2000$ psi press. rating, $200^{\circ} \mathrm{C}$ maximum temperature.

$1 / 4^{n}$ Nupro check valve, 3000 pressure rating, 10 psi cracking pressure.

Linde cylinder regulator, 0-7000 psi outlet pressure, 7000 psi maximum inlet. 
$\mathrm{R} 2,3$

1

R4

Back Pressure

Regulator

Sample Cylinders:

$\mathrm{Cl}$

$\mathrm{C} 2$

C3,4

Hydrogen

Reservoir

Liquid

Product

Collector

Liquid

Sampling

Tubes

Tubing:

$1 / 4^{n}$

$1 / 8^{n}$

Tube Fittings:

Gas and

Liquid Lines

Pressure

Gauge Lines

Pump:

P1

Liquid

Feed Pump

volume.

Transducer:

FT1
Linde cylinder regulator, 0-3500 psi outlet pressure, 3500 psi maximum inlet pressure.

Grove back pressure regulator, 100-2000 psi.

Hoke 1 liter sample cylinder, Monel construction, 5000 psi pressu rating.

Hoke 1 liter sample cylinder, Monel construction, 5000 psi pressure rating.

$1 / 4^{n}$ SS tube, seamless, $3^{n}$ long, $0.035^{n}$ thickness, 5900 psi pressure rating.

$1 / 4^{\prime \prime}$ O.D., $0.035^{\prime \prime}$ wall thickness, $3165 S$, seamless, 5900 psi pressure rating.

1/8" O.D., 0.028" wall thickness, 316SS, seainless, 9000 psi pressure rating.

Swagelok 316 SS, tubing fittings, pressure rating equal to or greater than that of tubing.

Ruska Metering Pump, $3 / 4$ hp, 110 volt, 60 cycle, single phase motor, 5000 psi working pressure, $350^{\circ} \mathrm{C}$ maximum operating temperature, 1000 cc cylinder

1/4" Hastings mass flow transducer (Model HS5KSSPX), 3000 psi pressure rating, $0-5000 \mathrm{sccm}$ flow range, 115 volt, 50 cycle, single phase power. 
Thermocouples:

TC1

TC2

Pressure Gauges:

$\mathrm{G} 1,2$

G3

G4

G5,6,7,8

G9

Heat Exchanger:

E1

Separators:

S1

S2

Gas Cylinders:

T1

$\mathrm{T} 2,3$
Reactor

Thermocouple

Furnace TC

Hydrogen

Supply Pressure

Reactor

Pressure

Collection

Vessel Pressure

Hydrogen

Supply Pressure

Back Pressure

Set Point

Condenser and

Cooler

Double pipe exchanger, $3 / 4^{n}$ O.D. outside tube and $1 / 8^{n}$ O.D. inside tube.
Omega chromel-alumel thermocouple, $1 / 8^{\prime \prime}$ O.D. $3165 S$ sheath.

Omega chromel-alumel thermocouple

Linde 0-7500 psi pressure gauge, 200 psi per division.

Heise 0-3500 psi pressure gauge, 5 psi per division.

Matheson 0-3000 psi pressure gauge, 100 psi per division.

Grove $0-4000$ psi pressure gauge, 100 psi per division
Stage I

Separator

Stage II

Separator

Hydrogen

Source

$n$
$1 / 2^{n}$ O.D. tube, $316 S S, 4000$ psi pressure rating, $0.049^{n}$ thickness.

3/4" O.D. tube, 316 SS, $0.065^{\prime \prime}$ thickness, 3487 psi pressure rating.

6000 psi Linde hydrogen cylinder.

2500 psi Union Carbide hydrogen cylinder. 
There are a number of differences between the earlier unit and the reactor now being constructed. The flow pattern through the new reactor system is completely different. Also, a high rate of flow $(-1.5 \mathrm{gpm})$, which is a much higher magnitude than that used in the earlier unit, will be maintained in the new system. This flow rate is necessary to evaluate both the free-radical control mechanism proposed in earlier reports and the gas-liquid contactor techniques that bave been developed. Other differences include a slurry-phase catalyst system (as opposed to a catalyst mounted in a spinning basket, Figure 4, P.4) and a new pumping system capable of higher flows. Our concern about the pumping system's capabilities is one reason why we are constructing and evaluating the reactor system in three stages.

The heat transfer demand of the new reactor system is much greater than that of the earlier unit. Because of higher flow rates and temperature cycling of liquefaction reactants, heat transfer rates will be comparatively higher. We have therefore evaluated three different types of heat transfer devices for the reactor: (1) an electrical induction furnace (Applied Test Systems model 3210), (2) a heat exchanger with a liquid heating fluid (Dowtherm, etc.), and (3) a sand bath heater. The first device is most appropriate for our small system because of its simplicity, ease of implementation, and ease of operation.

We are currently using a gallon autoclave (Autoclave Engineers model 74-4569) to preheat the reactants to near reaction temperature (between $300^{\circ} \mathrm{C}$ and $330^{\circ} \mathrm{C}$ ). During each pass of reactant through the reaction zone, an electrical furnace is used to preheat the reactant to near reaction temperature, and a sand bath heater is used to heat the reactant to the desired reaction temperature. A standard heat exchanger with water injection is used to quench the reactant to a lower temperature (reactivity level) prior to cycling back through the reactor. The Stage 1 Reactor System (Figure 1, P.1) will primarily be used to evaluate heat transfer techniques.

Prior to discussing briefly the Stage 2 Reactor System (Figure 11, P.21), we will review our 
goals and thereby discuss operating conditions to be used in the intended liquefaction reaction studies. On a related point, we will review some of our work with iron-based catalysts.

In the first part of our work with iron-based catalysts, we explored different possibilities for preparing a high hydrogenation activity catalyst. Naphthalene hydrogenation was used as a model reaction to rate the hydrogenation activities of different additives. Presulfiding iron oxdde with $\mathrm{H}_{2} \mathrm{~S}$ under controlled conditions rendered the highest hydrogenation activity, even greater than that of commercial CoMo/ $/ \mathrm{Al}_{2} \mathrm{O}_{3}$ catalyst sulfided with creosote oil and hydrogen. Sulfiding of $\mathrm{CoMo} / \mathrm{Al}_{2} \mathrm{O}_{3}$ catalyst with $\mathrm{H}_{2} \mathrm{~S}$ remarkably improved its initial hydroger,ation activity.

Nun erous researchers have studied the catalytic effect of indigenous coal minerals. Through gravity sepaiation, Mukherjee and Chowdhury (4) demineralized coal to isolate the effects of various mineral constituents on coal liquefaction. They found coal conversion to be directly related to the iron and sulfur content of the coal. In addition, they concluded that the iron, in the form of iron sulfide, acts as a hydrogen catalyst. In a study on hydroliquefaction of coal, Granoff et al. (5) observed that the addition of pyrite, pyrrhotite $\left(\mathrm{Fe}_{a \alpha} \mathrm{S}\right)$, and residue obtained from a previous run had a positive effect on both the $\mathrm{H}: \mathrm{C}$ ratio of the product and the hydrogen consumption. In a similar work at Auburn University, 75 different minerals and additives were screened for their catalytic activity on oil-producing reactions (6). All of the iron compounds were found to improve coal conversion and oil yield.

Moroni and Fischer (7), in a review of coal mineral catalysis research, found pyrite to be the major coal mineral in catalyzing coal liquefaction reactions. Also, they concluded that pyrite and its derivative, pyrrhotite, exhibit a dual functionality (hydrogenation and hydrocracking). This dual functionality is typical of most supported, hydrofining catalysts such as $\mathrm{CoMo} / \mathrm{Al}_{2} \mathrm{O}_{3}$.

In an earlier study (8), we compared the hydrogenation activity of various minerals for coal liquefaction reactions. The results of these exp:riments are presented in Figure 5. Of the minerals 
studied, pyrite was found to have a hydrogenation activity significanily higher than the others, Including pyrrhotite. On the other hand, the hydrodesulfurization (HDS) activity of pyrite was observed to be low, lower than that of pyrrhotite, for instance. These results agree with those of Granoff et al. (5). It should also be noted that the hydrogenation activity of pyrite increased as the particle size of the pyrite was reduced (8). Garg and Givens (9) have concluded that adding pyrite during coal liquefaction increases coal conversion and improves oll and gas production. Hydrogen consumption increases, and rebydrogenation of the process solvent is aided, with the addition of pyrite. We also found that the lower the final partial pressure of hydrogen in the reactor, the higher the hydrogenation activity of the catalyst. Thus, commercial $\mathrm{CoMo} / \mathrm{Al}_{2} \mathrm{O}$, catalyst was found to be the most active hydrogenation catalyst among materials tested.

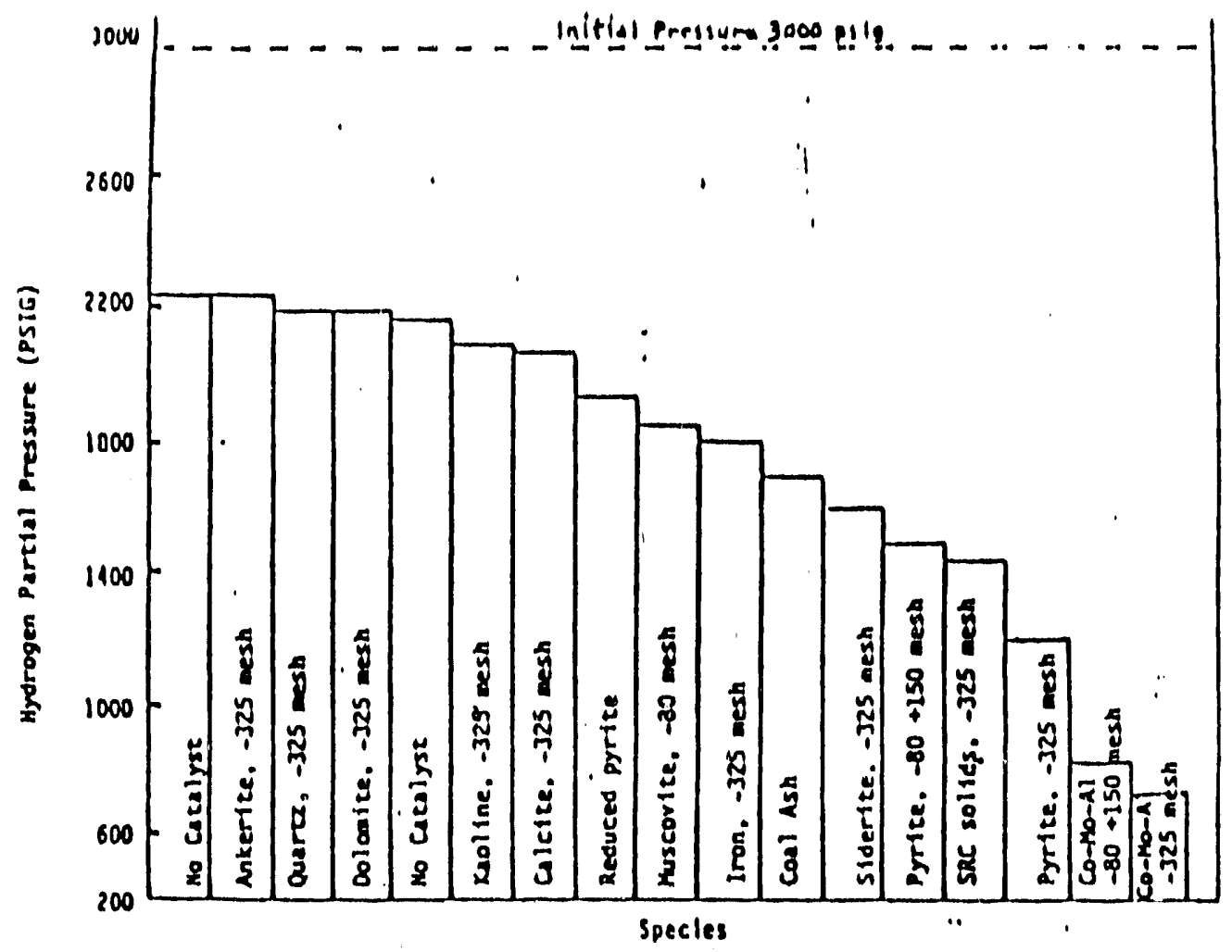

Figure 5: Comparison of Hydrogenation Activity of Catalyst 
Of all the mineral additives we screened, Fisher reagent grade pyrite was the only one that showed any significant naphthalene hydrogenation activity, even though its surface area was only 0.2 $\mathrm{m}^{2} / \mathrm{g}$. All other iroll-based additives showed only marginal improvement during thermal hydrogenation even though their surface areas were as high as $13.2 \mathrm{~m}^{2} / \mathrm{g}$.

In an effort to produce high surface area pyritic materials, the wet precipitation technique was most successful. But the high surface area pyritic catalyst produced $\left(11.8 \mathrm{~m}^{2} / \mathrm{g}\right)$ showed an insignificant activity for naphthalene hydrogenation. Reducing a Fisher reagent grade pyrite with hydrogen gas also showed improvements both in its surface area $\left(1.5 \mathrm{~m}^{2} / \mathrm{g}\right.$ versus $\left.0.2 \mathrm{~m}^{2} / \mathrm{g}\right)$ and in naphthalene hydrogenation activity. In-situ sulfiding of iron oxide with different sulfur sources like pyrite and thiophene did not appear helpful in improving the hydrogenation activity of $\mathrm{Fe}_{2} \mathrm{O}_{3}$.

We determined that sulfiding iron oxide with hydrogen sulfide gas prior to using it as a hydrogenation catalyst was the most effective technique for preparing the high hydrogenation activity mineral catalyst. A univariant search was made to determine the optimum sulfiding temperature while using both pure hydrogen sulfide and a mixture of hydrogen and hydrogen sulfide gas. Over the temperature range of $125^{\circ} \mathrm{C}$ to $500^{\circ} \mathrm{C}$, pure hydrogen sulfide proved more effective than a mixture of hydrogen and hydrogen sulfide for producing a sulfided iron oxide that was high in hydrogenation activity. The optimum sulfiding temperatures were determined to be $275^{\circ} \mathrm{C}$ when using pure hydrogen sulfide and $350^{\circ} \mathrm{C}$ when using a mixture of hydrogen and hydrogen sulfide (Figure 6). These catalysts showed $90 \%$ and $75.6 \%$ naphthalene conversions, respectively. The surface area of the catalyst varied significantly with sulfiding temperature: the higher the temperature, the lower the surface area (Figure 7). Also, the sulfiding temperature appeared to mildly affect only the form of the iron sulfide synthesized. Conversely, the $\mathrm{H}_{2} / \mathrm{H}_{2} \mathrm{~S}$ flow ratio had a more pronounced effect on the composition of synthesized iron sulfides. (Table 2 shows the effect of sulfiding conditions on the pyrite composition.) 


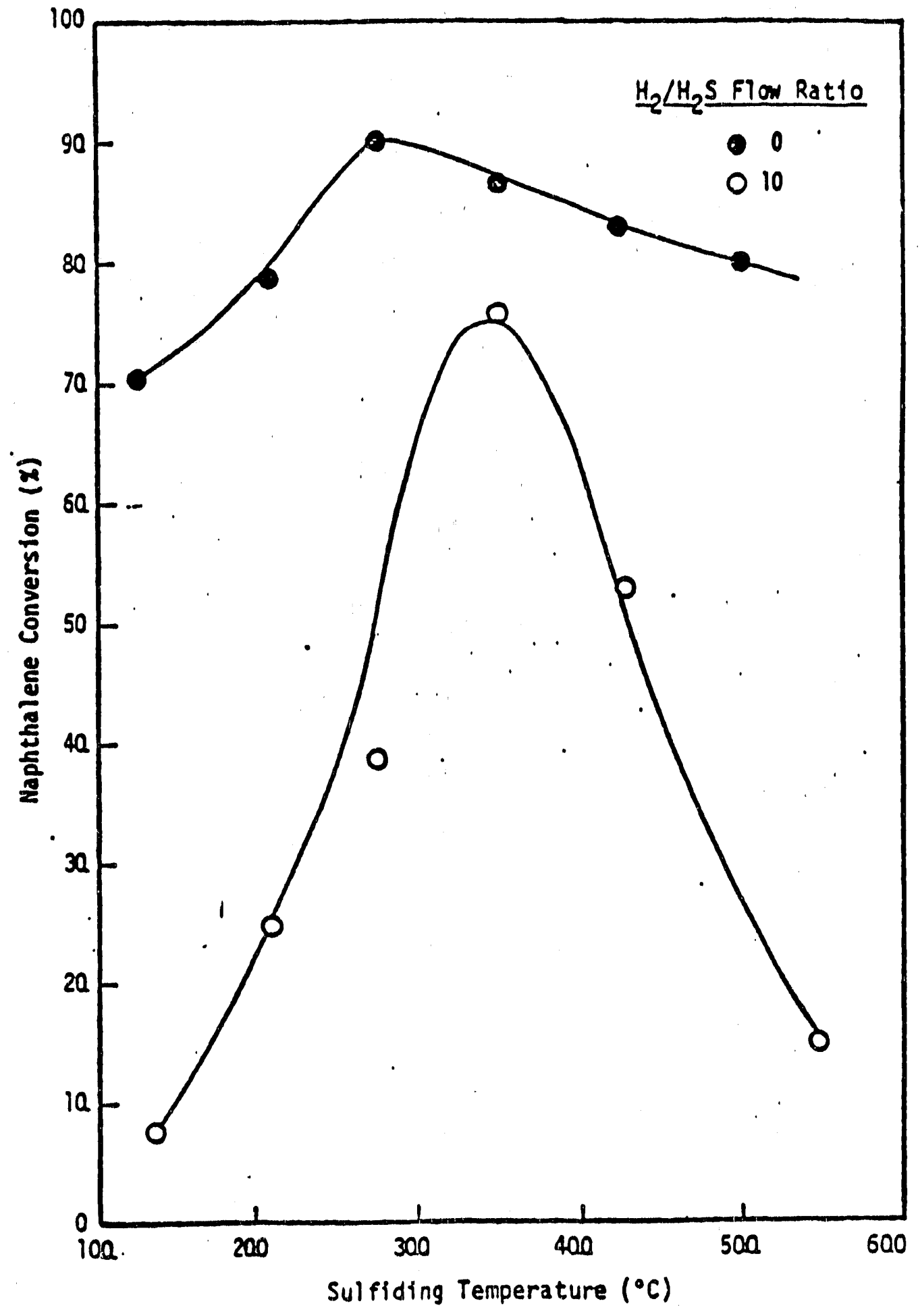

Figure 6: Naphthalenc Hydrogenation Activity of Presulfided Iron Oxide as a Function of Sulfiding Temperature 


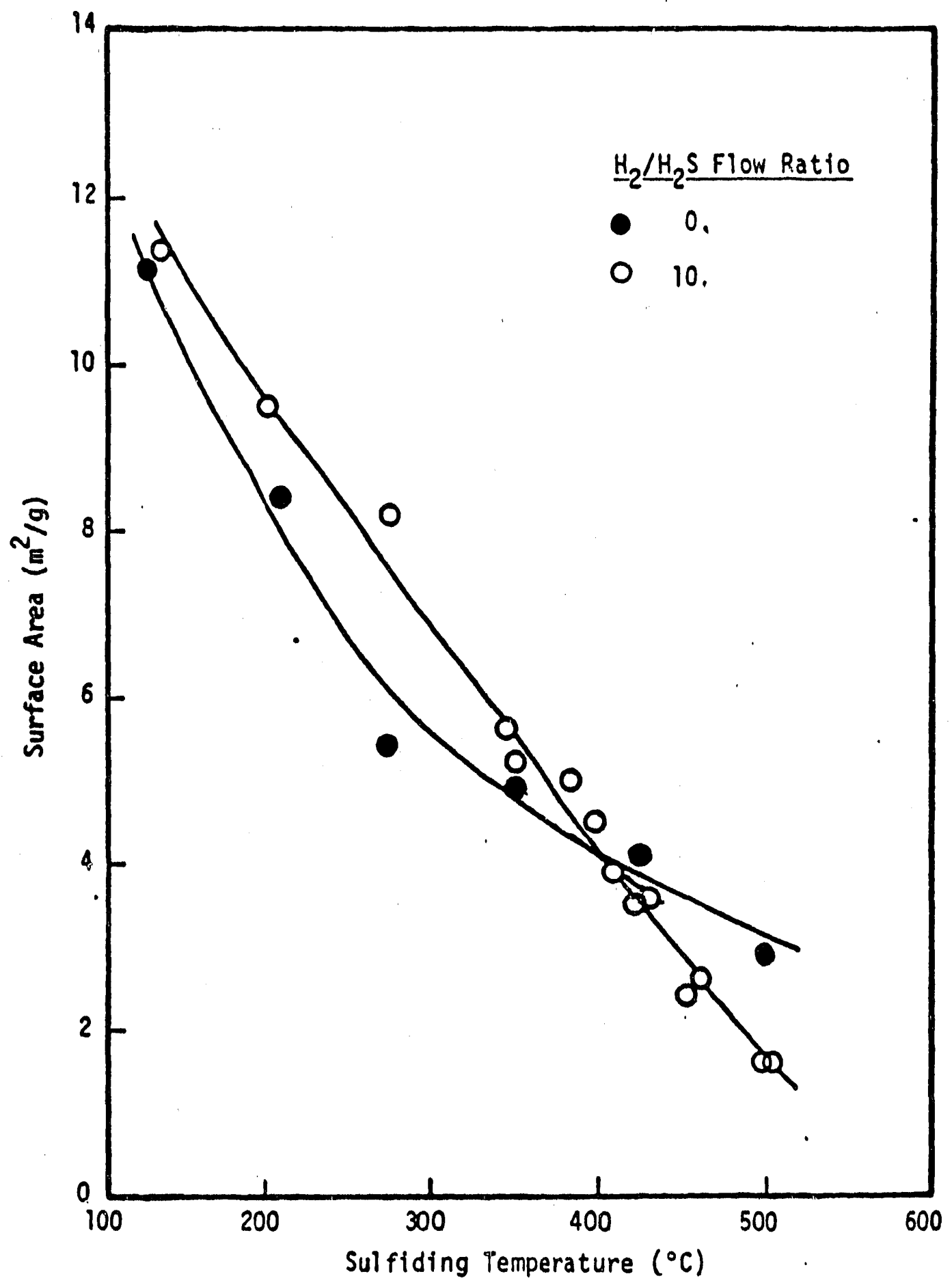

Figure 7: Effect of Sulfiding Temperature on the Surface Area of Presulfided Iron Oxide 
Table 2

Effect of Sulfiding Conditions on the Catalyst Composition

\begin{tabular}{|c|c|c|c|}
\hline \multirow{2}{*}{$\begin{array}{c}\mathrm{H}_{2} / \mathrm{H}_{2} \mathrm{~S} \\
\text { Flow Ratio }\end{array}$} & \multirow{2}{*}{$\begin{array}{c}\text { Sulfiding } \\
\text { Temperature } \\
\left({ }^{\circ} \mathrm{C}\right)\end{array}$} & \multicolumn{2}{|c|}{ Catalyst Composition } \\
\hline & & Major Phase & Minor Phase \\
\hline \multirow{3}{*}{0} & 125 & $\begin{array}{c}\mathrm{Fe}_{2} \mathrm{O}_{3} \\
\text { (crystalline) }\end{array}$ & $\mathrm{FeS}_{2} \mathrm{Fe}_{3} \mathrm{~S}_{4}$ \\
\hline & 275 & $\mathrm{FeS}_{2}$ & $\mathrm{Fe}_{2} \mathrm{O}_{3}(<1 \%)$ \\
\hline & 425 & $\mathrm{FeS}_{2}$ & $\mathrm{Fe}_{3} \mathrm{O}_{4}$ \\
\hline \multirow{3}{*}{10} & 135 & $\begin{array}{c}\mathrm{Fe}_{2} \mathrm{O}_{3} \\
\text { (crystalline) }\end{array}$ & $\mathrm{Fe}_{3} \mathrm{~S}_{4}$ \\
\hline & 211 & $\mathrm{Fe}_{\boldsymbol{y}} \mathrm{S}_{4}$ & $\mathrm{Fe}_{2} \mathrm{O}_{3}$ \\
\hline & 400 & $\begin{array}{c}\text { FeS } \\
\text { (Troilite) }\end{array}$ & $\mathrm{Fe}_{3} \mathrm{O}_{4}$ \\
\hline
\end{tabular}

The high surface area $\left(>9 \mathrm{~m}^{2} / \mathrm{g}\right)$ presulfided iron oxide samples were pyrophoric. A loss in hydrogenation activity with overnight exposure to air was observed even for the catalyst with a surface area of $5.18 \mathrm{~m}^{2} / \mathrm{g}$, indicating deactivation of the catalyst with surface oxidation. Hence, we concluded that the procedure for handling pyritic catalysts was important in maintaining activity. The pyritic catalysts were subsequently handled in an inert atmosphere of nitrogen.

Hydrogenation activity of a CoMo/ $\mathrm{Al}_{2} \mathrm{O}_{3}$ catalyst was found to be a strong function of its sulfur content: the higher the sulfur content, the higher the activity (Table 3). The catalyst sulfided with pure $\mathrm{H}_{2} \mathrm{~S}$ possessed a higher sulfur content ( 9.48 wt\% sulfur) than the catalyst sulfided with creosote oil and hydrogen (2.76 wt\% sulfur). Also, the pseudo-first-order rate constant for naphthalene hydrogenation was determined to be about 6 times greater for the catalyst with higher sulfur content (3.241 $\mathrm{g}$ liquid/g catalyst/min) than for the one with lower sulfur content $(0.505 \mathrm{~g}$ liquid/g catalyst/min). 


\begin{tabular}{|c|c|c|c|c|c|c|}
\hline \multicolumn{7}{|c|}{$\begin{array}{l}\text { Table } 3 \\
\text { Change in Naphthalene Hydrogenation Activity of } \mathrm{CoMo} / \mathrm{Al}_{2} \mathrm{O}_{3} \text { Catalyst } \\
\text { with Degree of Sulfiding }\end{array}$} \\
\hline \multicolumn{7}{|c|}{ Activity Test Conditions } \\
\hline $\begin{array}{l}\text { Sulfiding } \\
\text { Technique }\end{array}$ & $\begin{array}{l}\text { Surface } \\
\text { Area* } \\
\left(\mathbf{m}^{2} / g\right)\end{array}$ & $\begin{array}{l}\text { Sulfur } \\
\text { Content } \\
\text { (wt \%) }\end{array}$ & $\begin{array}{l}\text { Catalyst } \\
\text { Loading } \\
\quad(\mathrm{g})\end{array}$ & $\begin{array}{l}\text { Reaction } \\
\text { Time } \\
\text { (min) }\end{array}$ & $\begin{array}{l}\text { Naphthalene } \\
\text { Conversion } \\
(\%)\end{array}$ & $\begin{array}{l}\text { Pseudo-1st } \\
\text { Order Rate } \\
\text { Constant } \\
\text { (g liquid/g } \\
\text { cat } / \mathrm{min} \text { ) }\end{array}$ \\
\hline Garg's & 300 & 2.76 & 0.50 & 30.0 & 78.0 & 0.505 \\
\hline $\begin{array}{c}\text { Present } \\
\text { Work }\end{array}$ & 300 & 9.48 & 0.25 & 15.0 & 91.2 & 3.241 \\
\hline
\end{tabular}

- Before Sulfiding

Activity Test Conditions: $5 \mathrm{~g}$ reaction mixture (10 wt\% naphthalene in hexadecane), $410^{\circ} \mathrm{C}$, $1250 \mathrm{psig} \mathrm{H}_{2}$ at room temperature, $860 \mathrm{rpm}, 2$ steel ball agitators, $15 \mathrm{ml}$ reactor.

For $\mathrm{CoMo} / \mathrm{Al}_{2} \mathrm{O}_{3}$ catalysts, we observed that changes in the bydrogenation activity of the catalyst resulting from differences in the activation procedure alters the product distribution of the Elkhorn \#3 coal. In the temperature range of $425^{\circ} \mathrm{C}$ to $500^{\circ} \mathrm{C}$, the $\mathrm{CoMo} / \mathrm{Al}_{2} \mathrm{O}_{3}$ catalyst sulfided with pure $\mathrm{H}_{2} \mathrm{~S}$ gas (resulting in $9.48 w t \% \mathrm{~S}$ ) showed better catalyst selectivity than the catalyst sulfided with creosote oil and $\mathrm{H}_{2}$ (resulting in $2.76 \mathrm{wt} \% \mathrm{~S}$ ). From the naphthalene hydrogenation experiments, it was determined that, in terms of the pseudo-first-order rate constant, the hydrogenation activities of these catalysts are $3.241 \mathrm{~g}$ liquid/g catalyst/min and $0.505 \mathrm{~g}$ liquid/g catalyst/min, respectively. These results indicate that the rate limiting step is hydrogenation (Refer to Figures 8,9 , and 10).

Maximum catalyst selectivity was observed at $450^{\circ} \mathrm{C}$. It was postulated that, at higher temperatures, regressive reactions would occur. Below $450^{\circ} \mathrm{C}$, coal conversion was observed to be low, whereas the amount of product gases was reasonable ( $12 \mathrm{wt} \%$ of MAF coal) at $450^{\circ} \mathrm{C}$. Hence, 


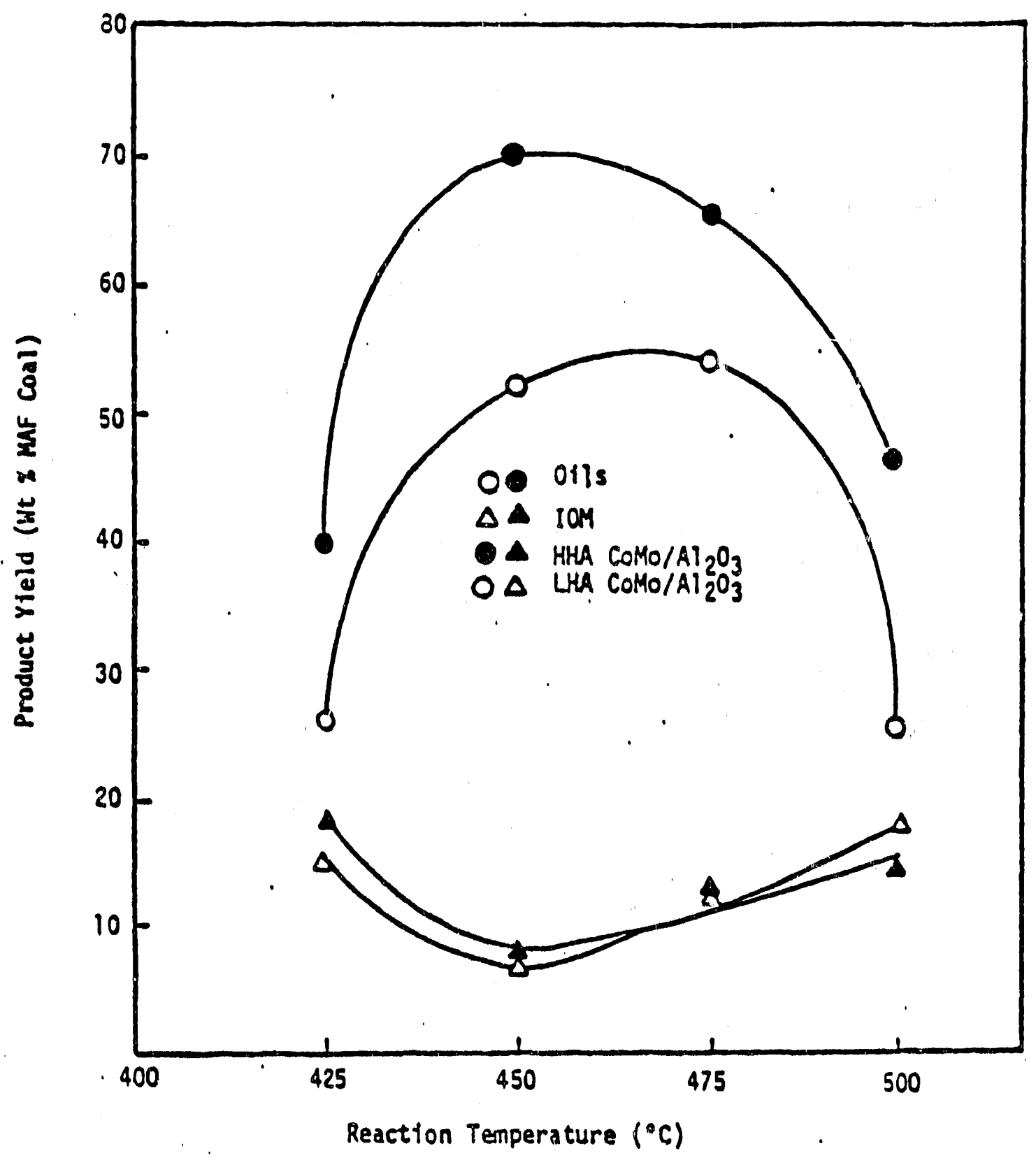

Figure 8: Comparison of Oils and IOM Yields Obtained with HHA and LHA $\mathrm{CoMO} / \mathrm{Al}_{2} \mathrm{O}_{3}$ 


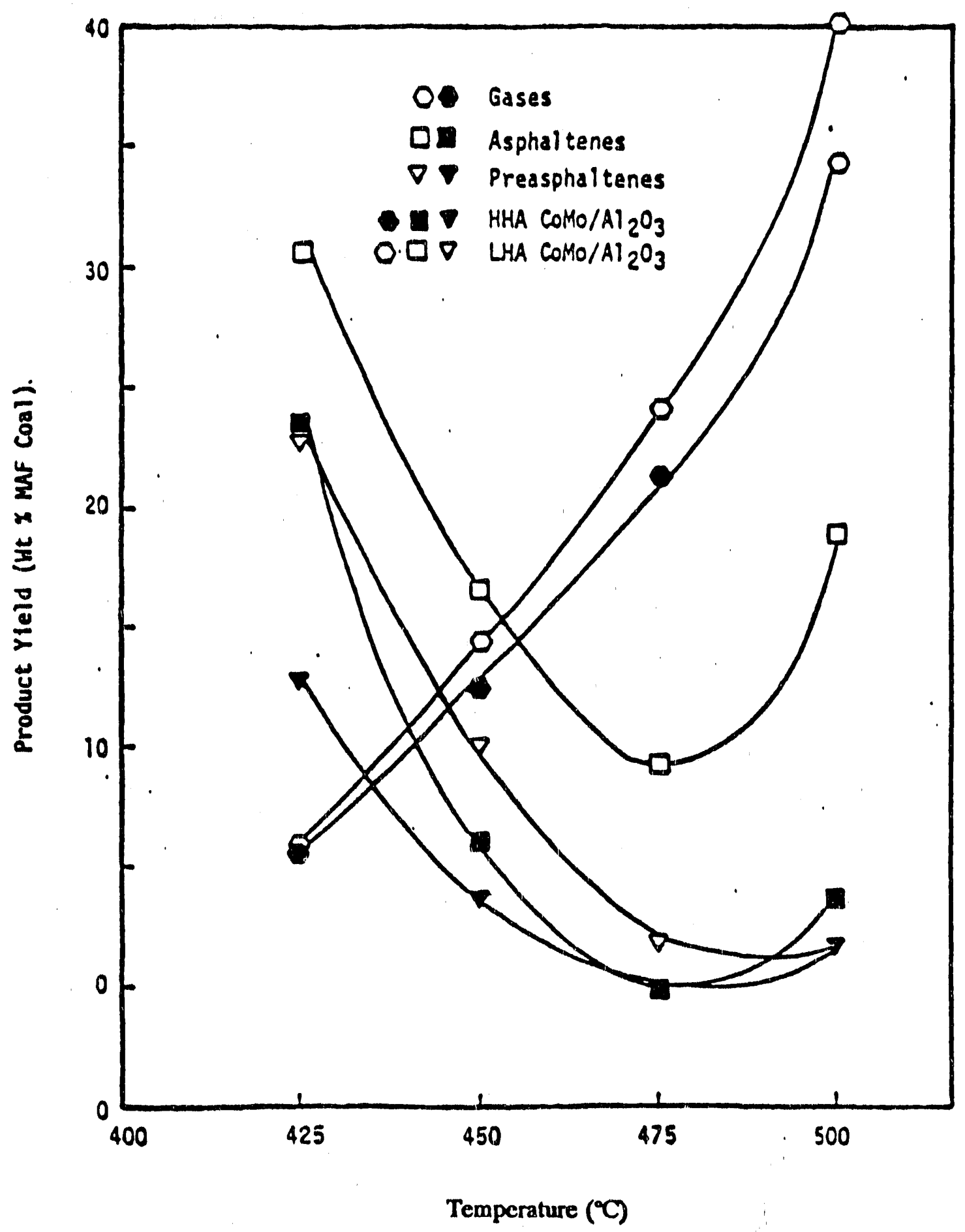

Figure 9. Comparison of Gas, Asphaltenes, and Preasphaltenes Yields Obtained with HHA and LHA CoMo/ $\mathrm{Al}_{2} \mathrm{O}_{3}$ 


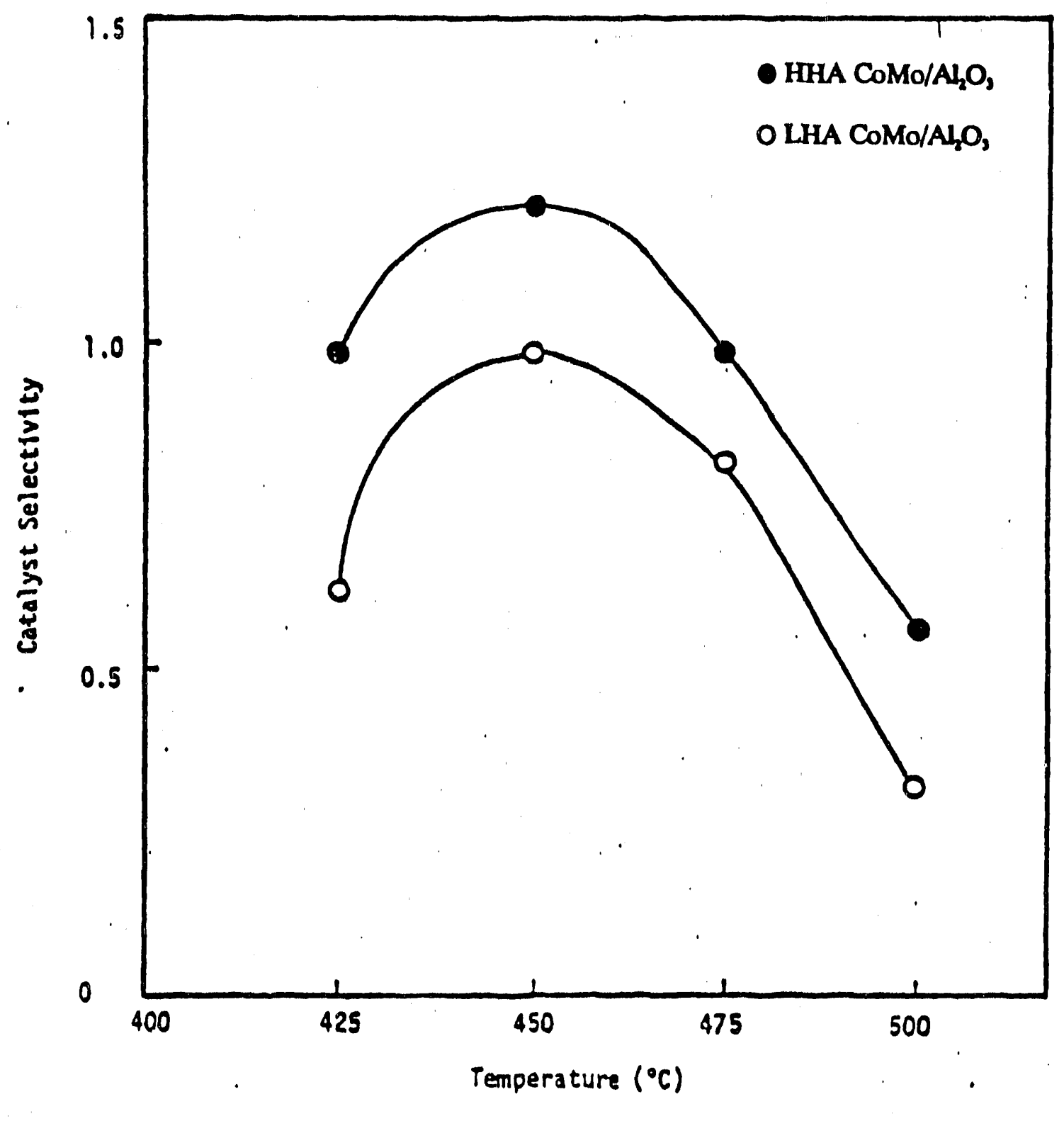

Figure 10: Comparison of HHA und LHA COMO/Al $\mathrm{O}_{3}$ Calalysts for Seloctivity in Coal Liquefaction 
$450^{\circ} \mathrm{C}$ was selected as the baseline reaction temperature.

With different mineral catalysts, essentially no difference in the product distribution of coal was observed even though the naphthalene hydrogenation experiments showed significant differences in their activities (Table 4). The reason behind this observation is not clear.

\begin{tabular}{|c|c|c|c|}
\hline \multicolumn{4}{|c|}{$\begin{array}{l}\text { Table } 4 \\
\text { Comparison of Presulfided Iron Oxide and Robena Pyrite } \\
\text { for Coal Liquefaction Catalysis (1) }\end{array}$} \\
\hline \multirow{2}{*}{$\begin{array}{c}\text { Yield } \\
\text { (Wt \%) }\end{array}$} & \multicolumn{3}{|c|}{ Additives } \\
\hline & None & $\begin{array}{l}\text { Presulfidied } \\
\text { Iron Oxide }\end{array}$ & $\begin{array}{c}\text { Robena Pyrite } \\
\text { (-325 mesh) }\end{array}$ \\
\hline Oils & $9 \pm 21$ & $42 \pm 1.4$ & $42 \pm 3.5$ \\
\hline Asphaltenes & $50 \pm 28$ & $42 \pm 1.9$ & $42 \pm 2.5$ \\
\hline Preasphaltenes & $13 \pm 1.0$ & $6 \pm 0.7$ & $7 \pm 0.0$ \\
\hline $\operatorname{IOM}^{(x)}$ & $28 \pm 1.5$ & $10 \pm 2.0$ & $9 \pm 1.0$ \\
\hline
\end{tabular}

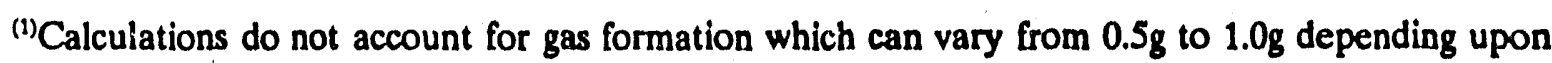
catalyst used.

${ }^{(2)}$ In determining IOM, pyrite has been assumed to form FeS at reaction conditions.

Reaction Conditions: $6 \mathrm{~g}$ solvent (AP.CPDU 121), $3 \mathrm{~g}$ coal (Elkhorn \#3), $1 \mathrm{~g}$ additive, $1250 \mathrm{psig} \mathrm{H}_{2}$ pressure at room temperature, $450^{\circ} \mathrm{C}, 60 \mathrm{~min}, 860 \mathrm{rpm}, 2$ steel balls, $46.3 \mathrm{ml}$ reactor.

Our study with pyritic catalysts demonstrates the basic problem to be addressed in coal research involving the reactor system now under construction: Why was there not a higher liquefaction activity with the higher hydrogenation activity pyritic catalysts? Two basic dominant mechanisms may be responsible for the insensitivity in liquefaction to improvements made in catalyst hydrogenation activity: (1) catalyst inhibition, and (2) rapid catalyst deactivation. Of these two mechanisms, rapid catalyst deactivation will be addressed in this study. We hypothesize that, once adequate mass transfer rates have been attained, rapid deactivation of the pyritic catalyst will not 
occur, resulting in a significant sensitivity in liquefaction rates to improvements in hydrogenation activity of pyritic catalysts.

Figure 11 is a schematic of the reactor system to be constructed in stage 2 . Here, the gasliquid contactors and gas-liquid recirculation system will be added, and batch-charged reaction studies will be performed. In stage 3, a continuous reactant feed system and a product collection system will be added.

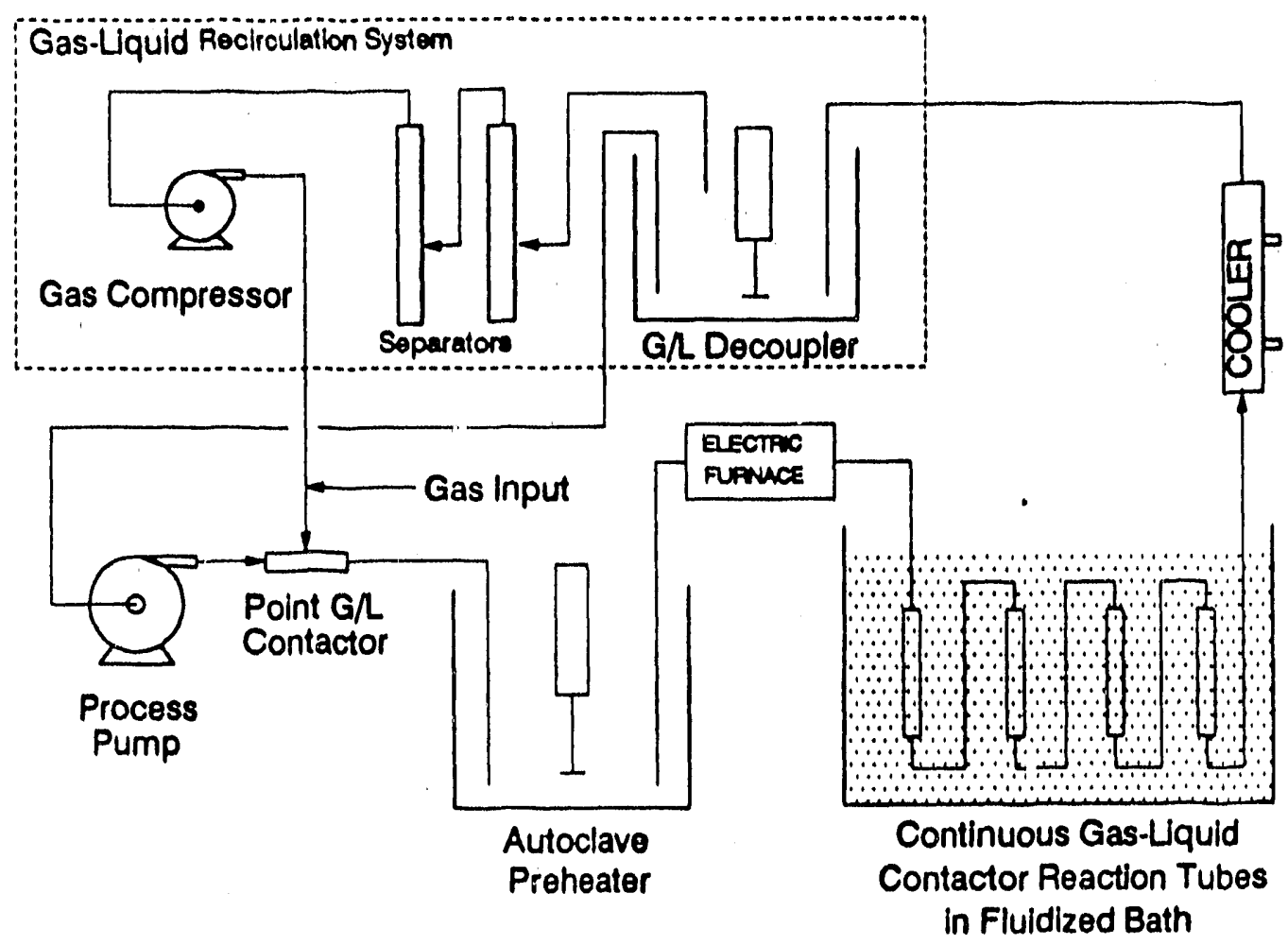

Figure 11: Schematic of Stage 2 Reactor System 


\section{Gas-Liquid Contactor Studiex.}

We are continuing to improve our gas-liquid contactor efficiency. We are currently focusing on point contactors and are studying responses in mass transfer rates to design parameter changes. As a result, we have improved the efficiency of our gas-liquid contactor by a factor of 4 , achieving a mass transfer coefficient of about 0.08 per second in a test system; with the previous contactor design, the highest rate had been only 0.016 per second. The following is a summary of findings from a literature survey for point contactors (10-15):

The rate of gas transfer to a liquid phase is generally represented by an overall mass transfer coefficient, $K_{a}$. It is well known that $K_{a}$ is a function of the bubble size, interfacial area, volume of liquid, the time of contact of gas with liquid, tank depth, agitation intensity, and other physical and chemical variables of the system.

Gas mass transfer in a system usually occurs in three different stages. First, about $80 \%$ of the total gas absorption occurs during bubble formation when the interface is created. Second, about $20 \%$ of the total absorption occurs during bubble rise. Thind, the bursting of the bubble at a surface, as well as surface turbulence, produces some absorption.

Smaller bubble sizes are important to mass transfer at low gas flow rate ranges because there are langer interfacial areas. In higher gas flow rate ranges, higher turbulence plays a more important role in mass transfer. Generally, $K$. increases with a decrease in tank depth and is proportional to gas flow rate.

In gas injector systems, $K$, also is proportional to gas holdup, gas velocity, liquid velocity, nozale diameter, bubble penetration depth, nozzle length-to-diameter ratio, and jet angle.

In the literature, the length-to-diameter ratio of an injector nozzle is typically designed to be over 50. Based on these findings, it appears that a series of multiple gas injections would be optimal: we are currently investigating whether this is true. 


\section{REFERENCES}

1. Nalitham, R.V., "Parametric and Kinetic Studies on Deactivation and Regeneration of Hydrotreating Catalysts in Solvent Refined Coal Upgrading Process and an Evaluation of the Liquid Vaporization Effects on Hydrotreater Performance," Doctoral dissertation, Auburn University, Al (1983).

2. "Kinetics of Coke Oxidation from Solvent Refined Coal Hydrotreating Solvents", Ind. Enp. Chem. Process Des. Dev. 1984.

3. "Effect of Phase Behavior on Hydrotreater Performance: Simulation and Experimental Verification", Ind. Eng. Chem. Process Des. Dev. 24, 598, 1985.

4. Murkherjee, D.K, and Chowdhury, P.B., Fuel, 55, 4 (1976).

5. Granoff, B., Thomas, M.G., Baca, P.M.0, and Noles, G.T., Am. Chem. Soc. Division Fuel Chem. Prepr. 23 (1), 23 (1978).

6. Huang, W.J., Curtis, C.W., Guin, J.A., Clinton, J.H., Barwood, H.L., and Tarrer, A.R., "Effects of Coal Minerals, By-Product Metallic Wastes and Other Additives on Coal Liquefaction." Presented at the national AIChE meeting, Houston (April 1981).

7. Moroni, E.C., and Fischer, R.H., "Disposable Catalysts for Coal Liquefaction." Presented at the 179th national ACS meeting, Houston (March 1980).

8. Guin, J.A, Tarrer, A.R., Prather, J.W., Johnson, D.R., and Lee, J.M., Ind. Eng. Chem. Process Des. Dev. 17, 118 (1978).

9. Garg, D., and Givens, E.N., Ind. Eng. Cliem. Process Des. Dev. 21, 113 (1982).

10. Funatsu, K., Y-C. Hsu, M. Noda, and S. Sugawa, "Oxygen Transfer in the Water-jet Vessel", Chem. Eng. Comm. 73, 121-139, 1988.

11. Funatsu, K., Y-C. Hsu, and T. Kamogawa, "Gas Holdup and Gas Entrainment of a Plunging Water Jet with a Constant Entrainment Guide", Can. J. Chem. Eng. 66, 19-28, 1988.

12. Ohkawa, A., D. Kusabirake, Y. Shiokawa, N. Sakai, and M. Fujii, "Flow and Oxygen Transfer in a Plunging Water Jet System Using Inclined Short Nozzles and Performance Characteristics of its System in Acrobic Treatment of Wastewater", Biotech. Bioeng., 28, 1845-1856, 1986.

13. Bin, A. K, "Comment on Article: Gas Holdup and Gas Entrainment of a Plunging Water Jet with a Constant Entrainment Guide", Can. J. Chem. Eng. 67, 699-701, 1989.

14. Prokop, A, P. Janik, M. Sobotka, and V. Krumphanzh, "Hydrodynamics, Mass Transfer, and Yeast Culture Performance of a Column Bioreactor with Ejector", Biotech. Bioeng., 25 , 1147-1160, 1983. 
15. Eckenfelder, W. W., "Process Design of Aeration Systems for Biological Waste Treatment", \$2(7), 286-292, 1956. 


\title{
Project III.4 (A) Catalytic Hydropyrolysis - Catalysts, Coals, and Liquid Product Quality. (B) Energized Coal Liquefaction
}

\author{
L.L. Anderson, J. Jaturapitpornsakul, W.-H. Yuan \\ University of Utah
}

1. Catalytic Hydropyrolysis - Catalysts, Coals, and Liquid Product Quality (J. Jumpol)

Catalytic hydropyrolysis of Blind Canyon coal (HVA, Utah) has been studied. Either iron, nickel or molybdenum have been used as catalysts in one-catalyst hydropyrolysis. In co-catalyst runs, iron or nickel was employed as a promoter for molybdenum. The first set of experiments was performed with molybdenum only to examine effects of ultrasonic impregnation and of coal pre-extraction on liquid yields. The second set was designed to compare catalytic activities of either iron or nickel to molybdenum and to investigate the possibility of increases in activity of molybdenum promoted by iron or nickel.

The Blind Canyon coal, obtained from Argonne Premium Samples, was demineralized with $\mathrm{HF}, \mathrm{HCl}$ and then with $\mathrm{HF}$ again. Iron (III) chloride hexahydrate, nickel (II) nitrate hexahydrate and ammonium tetrathiomolybdate were used as precursors for iron, nickel and molybdenum catalysts, respectively. The molybdenum salt was introduced to coal by either ultrasonic impregnation or incipient wetness impregnation, both in aqueous solution. In all other experiments, incipient wetness was used as impregnation method. In co-catalyst runs, the coal was impregnated with molybdenum salt first, dried and then impregnated with iron or nickel salt.

All the experiments were conducted in tubing bomb reactors heated by a fluidized sand bath. The reaction time was 1 hour and the reaction pressure was 2000 psig. The reaction temperature was $350^{\circ} \mathrm{C}$. The THF soluble and insoluble yields of these experiments are shown in Table 1. 
Table 1

Yields from Catalytic Hydropyrolysis of Blind Canyon Coal

\begin{tabular}{clcc}
\hline \multirow{2}{*}{ Run } & Catalyst & \multicolumn{2}{c}{ (Wt.\% of maf coal) } \\
THF insol. & THF sol.* \\
\hline \#2 & -none- Blind Canyon Coal & 76 & 22 \\
$\# 3$ & -none- Pre-extracted coal & 72 & 28 \\
$\# 4$ & 0.05\% Mo (ultrasonic impreg.) & 52 & 40 \\
$\# 5$ & 0.05\% Mo (Pre-extr. ultra. impreg.) & 59 & 39 \\
$\# 6$ & 0.05\% (Pre-extr. incip. impreg.) & 52 & 42 \\
$\# 7$ & $1.00 \% \mathrm{Fe}$ & 63 & 34 \\
$\# 8$ & $0.01 \% \mathrm{Fe}$ & 68 & 27 \\
\hline
\end{tabular}

*The difference between 100 and the sum of THF Insoluble and THF Soluble is gas + losses.

For demineralized Blind Canyon coal, the liquid yield from hydropyrolysis with molybdenum obtained from ultrasonic impregnation run was about the same as that obtained from incipient wetness impregnation. Pre-extraction of coal befure catalyst impregnation resulted in lower yields of liquids. This indicates the important role of pre-extracts in catalytic hydropyrolysis. From this set of experiments, molybdenum sulfide was shown to be a good catalyst for coal liquefaction even at low concentrations and relatively low temperatures.

In the iron catalyst runs, iron exhibited some catalytic activities at $1.0 \mathrm{wt} \%$ and no activity at $0.01 \mathrm{wt} \%$. However, iron was shown to be a good promoter for molybdenum at these reaction conditions, even at very low concentrations of iron. The amount of char produced was $41 \%$ from $1 \% \mathrm{Fe} / 0.05 \%$ Mo run and $43 \%$ for $0.01 \% \mathrm{Fe} / 0.05 \%$ Mo run, compared to $52 \%$ from $0.05 \%$ Mo-only run. When used alone, nickel had about the same activity as iron in these hydropyrolysis reactions. Nickel also was a good molybdenum promoter as the char yield indicated - $46 \%$ from $1 \% \mathrm{Ni} / 0.05 \% \mathrm{Mo}$ and $45 \%$ from $0.01 \%$ $\mathrm{Ni} / 0.05 \% \mathrm{Mo}$. At this temperature $\left(350^{\circ} \mathrm{C}\right)$, iron is a better choice as a catalyst promoter than nickel because of its much lower cost and also because it promotes the effect of molybdenum slightly better.

\section{Energized Coal Liquefaction (W.H. Yuen)}

Coal liquefaction by solvent extraction accompanied with the application of ultrasonic energy in solvent extraction of coal has been studied. The dissolution of coal is clearly improved by the application of ultrasonic energy, but the magnitude of improvement depends on the properties of the solvent used.

20-mesh Blind Canyon high volatile coal (PSOC-1503) was used as feed in a series of ultrasonic solvent extraction experiments. The results showed that when an organic base 
component was present in the solvent, the combined effect of ultrasound with the special coal-swelling effect by the solvent could give high values of coal dissolution. For example, when a solvent mixture composed of pyridine and methanol was used in ultrasonic extraction, the maximum coal dissolution at $63^{\circ} \mathrm{C}$ was about $20.4 \%$ (of the feed). But if the solvent mixture was composed of pyridine, methanol and tetra-n-butylammonium hydroxide (i.e., TBAH), the ultrasonic solvent extraction value at $63^{\circ} \mathrm{C}$ was increased to $44.1 \%$. Since TBAH only assists in coal swelling but not in dissolving, the increased yield is likely due to property changes in the bituminous coal structure. The distance between coal laminae could be increased in the swelling process or the strength of the Van der Waals or hydrogen bonds between coal laminae could be affected. These changis could cause the coal to become much easier to attack by the energized solvent.

The best extraction yield was obtained with an ultrasonic tip intensity of $88.57 \mathrm{w} / \mathrm{cm}^{2}$; the reaction temperature was $63^{\circ} \mathrm{C}$ and the solvent was compused of pyridine $(67.5 \%$ by wt\%), methanol (17.5\%), TBAH (6.5\%) and water (8.5\%).

More research by using similar solvents is in progress. Solvents which do not contain water tend to give higher extraction yields in the experiments done so far.

Data extracted from the described series of tests are listed in the following table. The reaction conditions were as follows:

Ultrasonic Tip Intensity (if applied): 88.57 watt $/ \mathrm{cm}^{2}$.

Reaction Temperature: $63^{\circ} \mathrm{C} /$

Solvent/Coal Ratio: $30.0 \mathrm{ml} / \mathrm{g}$ coal.

Solvent component: Pyridine/Methanol/Water/TBAH $=67.5 / 17.5 / 6.5 / 8.5$

$\begin{array}{ccc}\text { Reaction Time (min.) } & \text { Ultrasound Energized } & \text { Coal Extracted (\%) } \\ 15 & \text { No } & 10.1 \\ 30 & \text { No } & 13.5 \\ 60 & \text { No } & 15.2 \\ 90 & \text { No } & 15.3 \\ 15 & \text { Yes } & 20.6 \\ 30 & \text { Yes } & 38.1 \\ 60 & \text { Yes } & 44.0 \\ 90 & \text { Yes } & 44.1\end{array}$

Data needed to understand the difference in yield include analysis and characterization of the soluble products obtained with and without ultrasonic fields present. An evaluation also needs to be done on the costs involved in obtaining higher yields by the use of ultrasound versus conducting the extractions at higher temperatures and pressures. The kinetics of energized extraction and extraction without ultrasound are shown in Figure I. Additional kinetic data are being obtained along with evaluation of the liquids obtained during 
these extraction runs. These data should help us to understand the variables which control the liquefaction process and the reactions which produce the liquid products. 


\section{Task IV}

Novel Catalysts for Coal Liquefaction

Program Coordinators: Christine Curtis and Dady Dadyburjor 


\section{Project IV.1 Catalytic Dehydrogenation of Model Compounds in Relation to Coal Liquefaction \\ I. Wender, J. Tierney, and G. Holder SOMMARY}

Experiments in the last quarter on the palladium catalyzed dehydrogenation of the model hydroaromatic compound, 9,10dihydroanthracene $(9,10-\mathrm{DHA})$ have revealed that the dehydrogenation reaction depends, to various extents, on a number of factors. Attempts have been made to delineate the relative importance of these factors, which are interrelated.

We have found that 9,10-DHA rapidly loses hydrogen gas when heated with palladium catalysts in a high boiling $\left(385^{\circ} \mathrm{C}\right)$ solvent such as fluoranthene, which has a high reduction potential.

Pyrene (b.p.393 ${ }^{\circ} \mathrm{C}$ ) is a high boiling solvent with a lower reduction potential; it is a somewhat better solvent for the palladium catalyzed dehydrogenation of 9,10-DHA.

The boiling point of phenanthridine is $350^{\circ} \mathrm{C}, 35^{\circ} \mathrm{C}$ lower than fluoranthene and $43^{\circ} \mathrm{C}$ lower than pyrene; it has been found to be an excellent vehicle for the dehydrogenation of hydroaromatic compounds. This has been attributed to its low reduction potential. Phenanthrene and anthracene, for instance, boil at about the same temperature as phenanthridine, but have high reduction potentials and are poorer solvents for the dehydrogenation of 9,10-DHA and 9,10-dihydrophenanthrene (9,10-DHP).

Previous work has shown that $\mathrm{Pd}$ on $\mathrm{CaCO}_{3}$ (surface area of 12 $\mathrm{m}^{2} / \mathrm{g}$ ) is a poorer dehydrogenation catalyst than $\mathrm{Pd}$ on activated carbon (surface area of $1000 \mathrm{~m}^{2} / \mathrm{g}$ ). During this quarter, we established that the surface area of the palladium support was not important; both catalysts, when prereduced in hydrogen before reaction, gave essentially the same results. The state of the palladium is the deciding factor.

Previous work has shown that the use of a palladium catalyst for the dehydrogenation of both 9,10-DHA and 9,10-DHP in a lowboiling solvent such as quinaldine was unsuccessful; very little hydrogen gas was evolved even though it is likely that this solvent has a low reduction potential. 
It would be advantageous to use a low-boiling solvent for the catalytic dehydrogenation of model compounds, of recycle oils and of coal. With such solvents, adduction reactions, hydrogen transfer between hydroaromatics and solvents and selectivity to dehydrogenation of only hydroaromatics are alj. likely to be improved. With this in mind, we decided to attempt to use a hydrogen acceptor, so that hydrogen would be transferred to the acceptor rather than be evolved as molecular hydrogen. We chose stilbene $\left(\mathrm{C}_{6} \mathrm{H}_{5} \mathrm{CH}=\mathrm{CHC}_{6} \mathrm{H}_{5}\right)$ as the hydrogen acceptor. Stilbene boils at $305^{\circ} \mathrm{C}$.

In the presence of equimolar amounts of 9,10-DHA and stilbene, 9-10-DHA was completely dehydrogenated to anthricene in phenanthridine with no evolution of $\mathrm{H}_{2} ;$ all of the hydrogen was transferred to the stilbene to yield bibenzyl (identified by GCMS). We were surprised, however, to find that $92.5 \%$ of the hydroaromatic hydrogen in 9,10-DHA was transferred to stilbene in two hours even when the low-boiling quinaldine (b).p. $248^{\circ} \mathrm{C}$ ) was used as the solvent; the remainder of the hydrogen (2.74) was evolved as $\mathrm{H}_{2}$ gas. The vehicle, 2-methylnaphthalene (b.p. $243^{\circ} \mathrm{C}$ ) was almost as effective as quinaldine; $93.4 \mathrm{f}$ of the hydroaromatic hydrogen was transferred to stilbene; $5.5 \%$ appeared as molecular hydrogen. No hydrogen was removed from 9,10-DHA if the catalyst (Pd/C) was omitted in the experiment using 2-methylnaphthalene. With quinaldine, in the absence of a catalyst, no $\mathrm{H}_{2}$ was evolved and only $0.9 \%$ was transferred to stilbene.

When 9,10-DHA was dehydrogenated in the presence of half the equivalent of stilbene, half the hydroaromatic hydrogen in 9,10-DHA was evolved, and half was transferred to stilbene. Results of some experiments are given in Table 1.

\section{Future Work}

(i) The use of stilbene as a hydrogen acceptor in the dehydrogenation of 9,10-DHA, 9,10-DHP and other hydroaromatics such as hexahydro- and octahydroanthracene will be explored. Another possible hydrogen acceptor such as dimethyl fumarate. will be used to compare it with stilbene as a hydrogen acceptor. 
Table 1. Palladium-Catalyzed Dehydrogenation of 9,10-DHA

\begin{tabular}{|c|c|c|c|c|c|c|c|}
\hline \multirow{2}{*}{ Run No. } & \multirow{2}{*}{ catalyst } & \multirow{2}{*}{$\begin{array}{l}\text { TLme, } \\
\text { Temp. }\end{array}$} & \multirow{2}{*}{$\begin{array}{c}\text { Vehicle } \\
4.0 \mathrm{~g}\end{array}$} & \multicolumn{2}{|c|}{ Feed ${ }^{b}$} & \multirow{2}{*}{$\begin{array}{c}\mathrm{H}_{2} \\
\text { Evolved } \\
\end{array}$} & \multirow{2}{*}{$\begin{array}{c}\text { Blbenzy } 1 \\
8\end{array}$} \\
\hline & & & & $\begin{array}{c}\text { DHA } \\
g\end{array}$ & $\begin{array}{c}\text { st } 11 \text { bene } \\
g\end{array}$ & & \\
\hline DH- 12 & $\mathrm{Pd} / \mathrm{C}^{\circ}$ & $\begin{array}{c}2 \mathrm{hrg} \\
350\end{array}$ & $\mathbf{P}$ & 0.2 & 0.2 & 0.0 & 100 \\
\hline$D H-18$ & $\begin{array}{c}\mathrm{Pd} / \mathrm{CaCO}_{3} \\
\text { Not } \\
\text { Red'sced }\end{array}$ & $\begin{array}{c}2 . h r s \\
248\end{array}$ & $\mathbf{Q}$ & 0.2 & 0.2 & 6.1 & 3.3 \\
\hline$D H-20$ & $\mathrm{Pd} / \mathrm{S}$ & $\begin{array}{c}2 \mathrm{hrg} \\
248 \\
\end{array}$ & $\mathbf{Q}$ & 0.2 & 0.2 & 2.7 & 92.5 \\
\hline $\mathrm{DH}-23$ & $\begin{array}{l}\mathrm{Pd} / \mathrm{CaCO}_{3} \\
\text { Reduced } \\
\end{array}$ & $\begin{array}{c}2 \mathrm{hrg} \\
248 \\
\end{array}$ & $\mathbf{Q}$ & 0.2 & 0.2 & 14.5 & 74.0 \\
\hline$D H-28$ & $\mathrm{Pd} / \mathrm{C}$ & $\begin{array}{c}2 \operatorname{hrg} \\
243\end{array}$ & $M P$ & 0.2 & 0.2 & 5.5 & 93.4 \\
\hline$D H-32$ & NONE & $\begin{array}{l}1 \mathrm{hr} \\
243 \\
\end{array}$ & MP & 0.2 & 0.2 & 0.0 & 0.0 \\
\hline$D H-33$ & NONY & $\begin{array}{c}1 \mathrm{hr} \\
248 \\
\end{array}$ & $\mathbf{Q}$ & 0.2 & 0.2 & 0.0 & 0.9 \\
\hline$D H-37$ & $\begin{array}{l}\mathrm{Pd} / \mathrm{CaCO}_{3} \\
\text { Reduced } \\
\end{array}$ & $\begin{array}{c}1 \mathrm{hr} \\
243 \\
\end{array}$ & $M P$ & 0.2 & 0.2 & 8.8 & 90.4 \\
\hline DH-39 & $\mathrm{Pd} / \mathrm{C}$ & $\begin{array}{c}1 \mathrm{HR} \\
248 \\
\end{array}$ & $Q$ & 0.3 & 0.15 & 50 & 50 \\
\hline \multicolumn{8}{|c|}{$\begin{array}{l}\text { a. P=Phenanthridine (reduction potential, } 1.58 \text { ) } \\
\text { Q=Quinaldine (reduction potential, } 1.56 \text { ) } \\
\text { MP=2-Methylnaphthalene (reduction potentlal, } 2.96 \text { ) } \\
\text { b. DHA and otilbene have the same molecular weight. } \\
\text { c. All Pd/C catalyotg were reduced in flowing } H_{2} \text {. }\end{array}$} \\
\hline
\end{tabular}

(ii) Recycle oils and coal will be catalytically dehydrogenated in the presence of a hydrogen acceptor, using lowboiling solvents.

(iii) The hydroaromatic compound, 9,10-dihydro-9,10dimethylanthracene, will be studied. It is expected that hydrogen will be evolved more slowly from this hindered hydroaromatic so that rates of dehydrogenation can be determined.

(iv) The catalytic dehydrogenation of several coals will be explored using quinaldine as the solvent in the presence of a hydrogen acceptor. The results will be compared with the palladium catalyzed dehydrogenation of coal in the higher boiling phenanthridine (Reggel, 1968; Heredy, 1985). 


\section{Reterences}

1. Heredy, L.A., McCoy L.R., Skowronski,R.P. and J.J. Ratto, "Final Report on the Chemistry of the Extractive Phase of Coal Liquefaction," US-DOE Contract No. N45569 (1985).

2. Reggel, L., Wender, I., and R. Raymond, "Catalytic Dehydrogenation of Coal III--Hydrogen Evolution as a Function of Rank," Fuel, Vol.47, 373-90 (1968). 


\title{
Project IV.2 Evaluation of Dispersion Methods For Slurry Phase Catalysts
}

\author{
Ying Tang and Christine W. Curtis \\ Cherr.ical Engineering Department \\ Auburn University
}

\section{LITERATURE REVIEW}

A literature review was conducted evaluating the effect of dispersion on the activity of iron catalysts in coal liquefaction. A short summary of some of the literature findings is given below.

Comparisons of the catalytic activities of iron and molybdenum catalysts used in reactions of model coal molecules showed high catalytic activity for $\mathrm{Fe}(\mathrm{CO})_{5}$ or $\mathrm{Fe}(\mathrm{CO})_{9}-\mathrm{S}$ for hydrogenation of aromatic species (Suzuki et al., 1989). However, $\mathrm{Mo}(\mathrm{CO})_{6}-\mathrm{S}$ more strongly promoted hydrogenation of polyarnmatic compounds as well as hydrogenation of some phenyl carbon bonds. The effect of dispersion methods had also been examined using iron catalysts in which an iron oxide $(\mathrm{FeOOH})$ precursor was dispersed onto the solid coal matrix (Cugini et al., 1991). Other iron systems, such as $\mathrm{Fe}_{2} \mathrm{O}_{3}$ dispersed on carbon black, iron incorporated into coal by impregnating with $\mathrm{FeCl}_{3}$ and to lignite by cation exchange and a sulfated $\mathrm{Fe}_{2} \mathrm{O}_{3}$, have been used as finely dispersed catalysts for coal liquefaction and characterized by Mossbauer and XAFS spectroscopy (Huffman et al., 1991). The relation between chemical structure and reactivity has not yet been established.

Finely divided iron (III) oxides and iron oxyhydroxides modified with either $\left(\mathrm{SO}_{4}{ }^{2}\right)$ or molybdate $\left(\mathrm{MoO}_{4}{ }^{2-}\right)$ anions used in coal liquefaction have shown increased coal conversion and selectivities to n-pentane soluble products (Pradhan et al., 1991). The effect of the pyrite to 
pyrrhotite ratio produced from ferric sulfide as a precursor on coal liquefaction has been studied. A definite effect of intermediate stoichiometry of the ratio is observed on the selectivity to lighter products at higher liquefaction temperatures (Stansberry et al., 1991).

\section{EXPERIMENTAL WORK}

This quarter, the experimental work focused on developing the laboratory skills of a new Ph.D. candidate and on his performing initial experiments using finely dispersed catalysts. Pruviously, the skills of gas chromatography and the basic skills of performing of high pressure, high temperature microtubular reactions were learned by this new coal researcher. This quarter, the student mastered the performance of catalytic microtubular reactions and the analysis of the reaction products from a catalytic reaction using a model system. To make the learning process more straightforward, a simple model compound, naphthalene, was used to test the activity of two catalysts, molybdenum naphthenate and iron acetylacetonate. Next quarter, higher molecular weight model compounds as well as coal will be reacted with these same catalysts. In order to analyze the products from these reactions, a septumless injector has been installed on the gas chromatograph. The Ph.D. candidate will also learn the coal liquefaction product fractionation procedure using a series of solvents.

The experimental work this quarter evaluated the amount of naphthalene hydrogenation occurring in the presence of molybdenum naphthenate and iron acetylacetonate. Both catalysts were used without sulfur, initially. Then sulfur was added to the reaction system and the effect of the added sulfur on the amount of naphthalene hydrogenation achieved was determined. 


\section{MATERIALS AND PROCEDURES}

Naphthalene hydrogenation reactions were performed using a 2.0 weight percent loading of naphthalene ( $99 \%$ purity from Aldrich) in hexadecane ( $98 \%$ purity from Aldrich). The catalysts used were molybdenum naphthenate, an oil soluble catalyst with a Mo content of 6 weight percent which was obtained from Shepherd Chemical Company. Iron acetylacetonate, (FeAcAc) 3 ( $99 \%$ purity from Kodak Eastman Company) a powder, was used as a precursor for an iron catalyst.

Batch hydrogenation reactions were performed in microtubular reactors. The reactors were charged with $4.0 \mathrm{~g}$ of a 2.0 weight percent solution of naphthalene with a catalyst, either Mo naphthenate or $\mathrm{Fe}(\mathrm{AcAc})_{3}$, premixed in the reactant solution at a level of 900 to $1000 \mathrm{ppm}$ Mo or $\mathrm{Fe}$ in the reactant solution. The reactions were conducted at $380^{\circ} \mathrm{C}$ for 30 minutes at an agitation rate of $550 \mathrm{cpm}$. The catalytic reactions were performed with and without the presence of excess sulfur. The catalyst precursor was sulfided in situ by elemental sulfur introduced at a stoichiometric ratio of 3 to 1 of $S$ to metal. Experiments were also performed with $\mathrm{Fe}(\mathrm{AcAc})_{3}$ at different ratios of $\mathrm{Fe}$ to $\mathrm{S}$.

\section{RESULTS AND DISCUSSION}

Naphthalene hydrogenation is a relatively simple reaction. The products that are typically formed under catalytic hydrogenation conditions are tetralin, decalin and $n$-butylbenzene. The arrount of each product obtained is dependent upon the reaction temperature, time, and hydrogen pressure as well as on the type and quantity of catalyst used.

Under thermal hydrogenation conditions, less than one mole percent naphthalene was converted to tetralin at $380^{\circ} \mathrm{C}$ as shown in Table 1 which is in agreement with previous research 
(Kim and Curtis, 1990). When Mo naphthenate was introduced, approximately $30 \%$ conversion of naphthalene to tetralin occurred. The addition of excess sulfur to the reaction system caused the conversion to nearly double, yielding more than $60 \%$ tetralin and a trace amount of decalin (Table 1).

Reactions of naphthalene hydrogenation were then performed with $\mathrm{Fe}(\mathrm{AcAc})_{3}$. When reacted at the same reaction conditions as Mo naphthenate, $\mathrm{Fe}(\mathrm{AcAc})_{3}$ resulted in much higher conversion of naphthalene to tetralin than did Mo naphthenate. Nearly $90 \%$ conversion was achieved. Neither decalin nor n-butylbenzene was produced during the reaction; hence, $\mathrm{Fe}(\mathrm{ACAc})_{3}$ showed a high selectivity for producing hydruaromatics at liquefaction conditions. However, under actual liquefaction conditions in the presence of coal and liquefied coal, sulfur will always be present. The reactions of $\mathrm{Fe}(\mathrm{AcAc})_{3}$ were performed with excess sulfur. The product distribution changed substantially in the presence of sulfur. The conversion of naphthalene decreased significantly from more than $90 \%$ with $\mathrm{Fe}(\mathrm{AcAc})_{3}$ to $6 \%$ with $\mathrm{Fe}(\mathrm{AcAc})_{3}$ and excess sulfur. The catalytic activity of $\mathrm{Fe}(\mathrm{AcAc})_{3}$ was severely poisoned by sulfur. This result is in contrast with that of Mo naphthenate whose activity was promoted by excess sulfur.

The effect of the amount of sulfur on the activity of $\mathrm{Fe}(\mathrm{AcAc})_{3}$ was examined as presented in Table 2. The amount of sulfur present was varied from a stoichiometric amount of 3 to 1 to 0.2 tr 1 sulfur to iron to produce $\mathrm{Fe}_{x} \mathrm{~S}$ where $\mathrm{x}$ is equal to 0.5 . The conversion of naphthalene was $6 \%$ with 3 to $1 \mathrm{~S}$ to $\mathrm{Fe}$ and decreased to $1.3 \%$ with a 1 to $1 \mathrm{~S}$ to $\mathrm{Fe}$. At lower $S$ amounts, no conversion of naphthalene occurred. Obviously, the amount of $S$ had a definite effect on the activity of the iron sulfide produced. The result leads to the supposition that 
different stoichiometries of iron sulfides are being produced, each of which has a different activity for hydrogenating naphthalene.

\section{CONCLUSIONS}

Initial experiments showed that naphthalene hydrogenation to tetralin can be accomplished successfully by the addition of an $\mathrm{Fe}(\mathrm{AcAc})_{3}$ precursor. The activity for hydrogenation of the Fe catalyst generated in situ was greater than the $\mathrm{MOS}_{2}$ generated in situ from Mo naphthenate and excess sulfur. The addition of sulfur to the $\mathrm{Fe}(\mathrm{AcAc})_{3}$ changed the character of the in situ generated Fe catalyst and reduced its activity for hydrogenation substantially. The amount of sulfur present in the reaction changed the activity of the in situ generated Fe catalyst, most likely because of a change in stoichiometry.

\section{FUTURE WORK}

The work planned for next quarter involves the use of higher molecular weight model compounds in reactions with $\mathrm{Mo}$ naphthenate, $\mathrm{Fe}(\mathrm{AcAc})_{3}$ and $\mathrm{Fe}$ naphthenate. The effect of sulfur and temperature on the activity of the catalysts will be tested. Also, catalysts will be generated in situ in a reaction with hexadecane only and then introduced into the model reactant system. The analysis procedure for fractionating coal liquids will be learned. 


\section{REFERENCES}

Cugini, A.V., Utz, B.R. Krustman, D., Hickey, R.I., Balsone, V. ACS Fuel Chemistry Division Preprints, 36(1), 91, 1991.

Huffman, G.P., Ganguly, B., Taghili, M., Huggins, F.E. and Shah, N., ACS Fuel Chemistry Division Preprints, 36(2), 561, 1991.

Kim, H., Curtis, C.W. Energy and Fuels, 4, 206-14, 1990.

Prahan, V.R., Holder, G.D., Tierney, J.W. and Wender, I., "Modified Iron Oxide Catalysts in Coal Liquefaction", Fifth Annual Technical Meeting, Consortium for Fossil Fuel Liquefaction Science, Lexington, Kentucky, August, 1991.

Stansberry, P., Zondlo, J.W., Yang, J., Stiller, A.H., Ragela, P., and Dadyburjor, D.B., "Ferric Sulfide as a Precursor for Coal Liquefaction", Fifth Annual Technical Meeting, Consortium for Fossil Fuel Liquefaction Science, Lexington, Kentucky, August, 1991.

Suzuki, T., Yamada, H., Sears, P.L. and Watanabe, Energy and Fuels, 3, 707, 1989. 


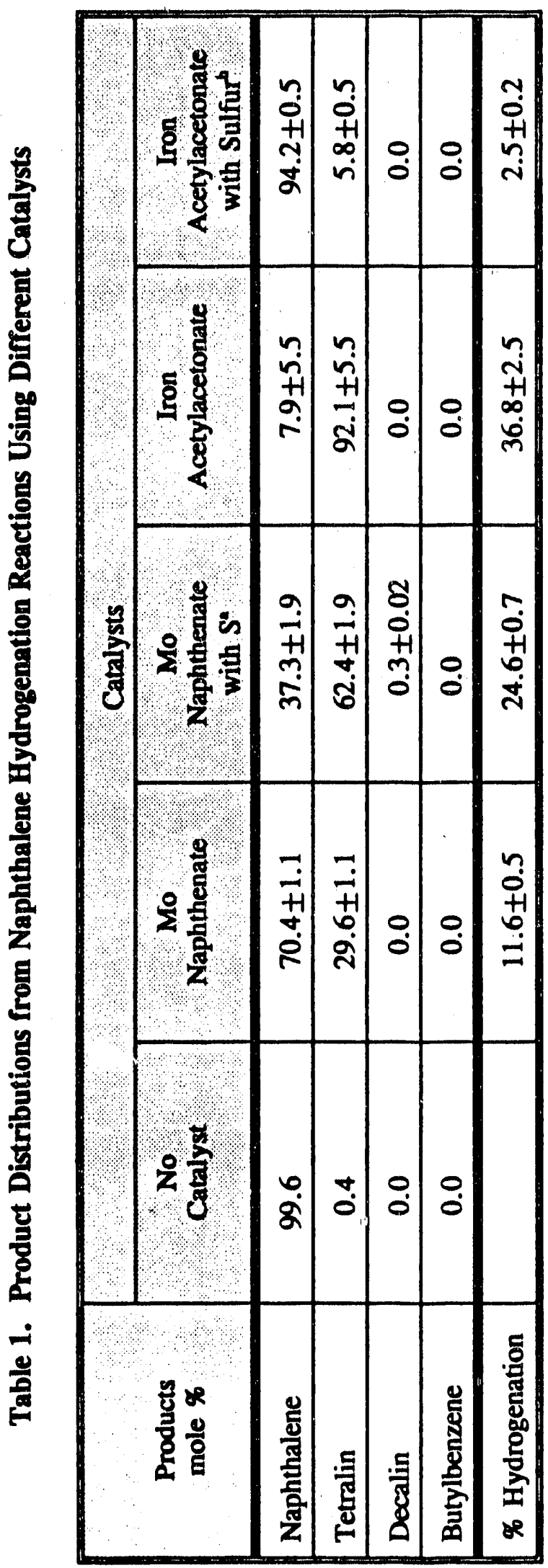




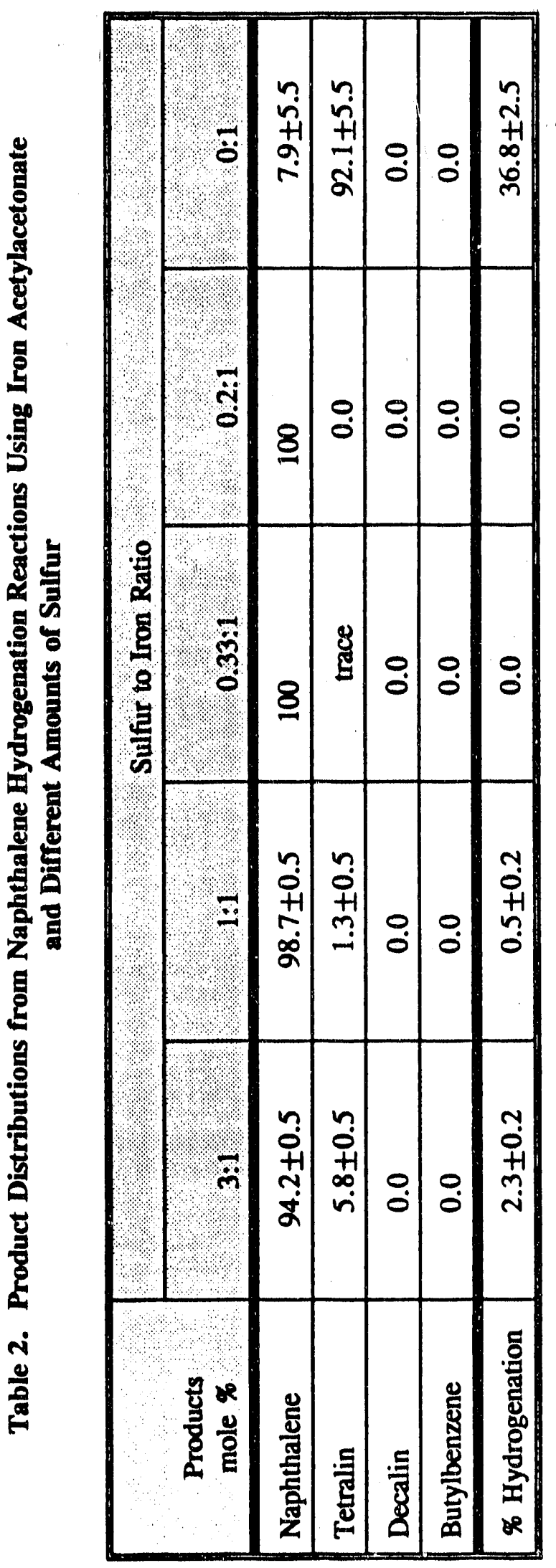




\title{
Project IV.3 Improved Catalysts for Coal Liquefaction and Co-Processing: New Initiative in Dispersed Slurry Phase Catalysts
}

\author{
J. Guin and $X$. Zhan \\ Auburn University
}

\section{INTRODUCTION}

In the last two quarters we began a new initiative dealing with slurry phase catalysts. These catalysts are being tested in a trickle flow reactor system using a fixed bed of small (powdered) particles. We are performing model compound experiments using iron based catalysts. Our objective is to determine conditions which maximize the activity for hydrogenation and cracking reactions. This should provide insight into how iron based catalysts function in coal liquefaction and how to pretreat the catalysts for maximum activity and desired selectivity. We have also placed an order for a pump which should allow our reactor to be adapted for colloidal catalyst particles in the feed.

\section{EXPERIMENTAL SECTION}

The reaction experiments were carried out in a CDS 900 trickle bed reactor system shown schematically in Figure 1, and which has been described in detail elsewhere ${ }^{[1]}$. A terminal computer 


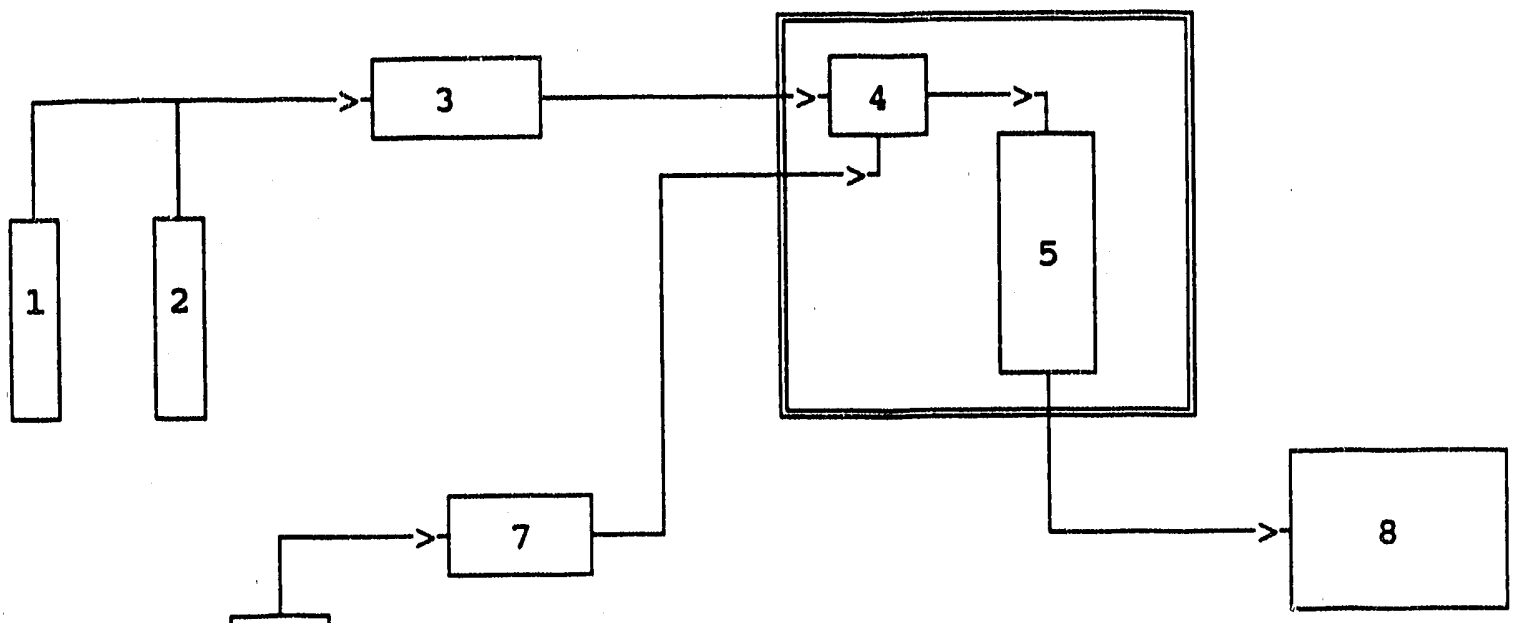

6

1. $\mathrm{H}_{2}$ cylinder

4. Gas-1iquid mixer

7. Liquid pump

2. $\mathrm{N}_{2}$ cylinder

5. Reactor tube

3. Gas flow controller

6. Liquid tank

8. Gas-liquid separator

Pigure 1 CDs 900 systam

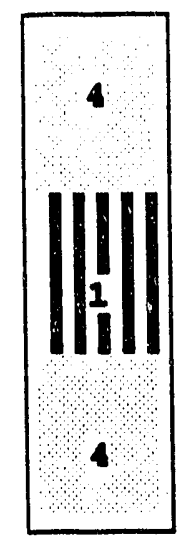

I. D. $=0.425^{\prime \prime}$

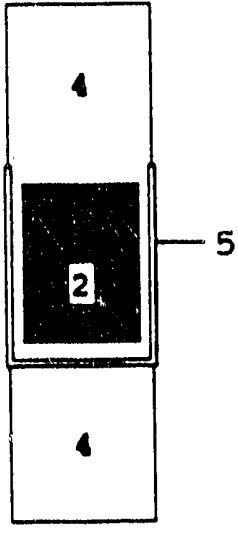

I. D. $=0.425^{\prime \prime}$

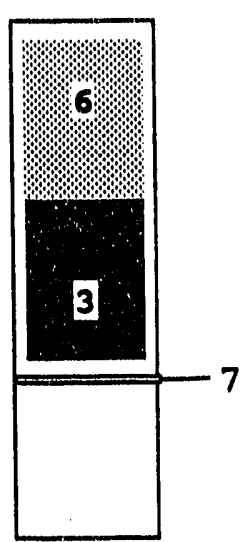

I.D. $=0.515^{\prime \prime}$

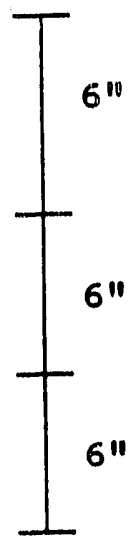

$6^{10}$

$(10$

"

1. $\mathrm{Ni}-\mathrm{MO} / \mathrm{Al}_{2} \mathrm{O}_{3}$ 2. $\mathrm{Fe}-1$ (Harshaw $\mathrm{Fe}_{2} \mathrm{O}_{3} / \mathrm{Al}_{2} \mathrm{O}_{3}$ )

3. $\mathrm{Fe}-2$ ( $\mathrm{Strem} \mathrm{Fe}_{2} \mathrm{O}_{3}$ ) 4. $3 \mathrm{~mm}$ glass bead 25. 400 mesh basket

6. 0.1-0.12 mm glass bead

7. $0.5 \mu \mathrm{m}$ ss frit

Pigure 2 Reactor structure 
is used to control all processing, charging, temperature, pressure and flow rate functions. The mixer is responsible for mixing the liquid and gas feeds to produce a dispersive stream entering the trickle bed reactor which is located in a 0.75 inch I.D. heating jacket. Three kinds of catalyst tested were Run $\# 1$, $\mathrm{NI}-\mathrm{MO} / \mathrm{Al}_{2} \mathrm{O}_{3}$ (Shell 324, 1/26", 10.58g), Fe-1 (Harshaw Chemical Co, supported $\mathrm{Fe}_{2} \mathrm{O}_{3}$ on $\mathrm{Al}_{2} \mathrm{O}_{3}, 35-60$ mesh, $10.94 \mathrm{~g}$ for Run \#2 and $10.79 \mathrm{~g}$ for Run \#3), and Fe-2 (strem Chemicals Inc, unsupported $\mathrm{Fe}_{2} \mathrm{O}_{3}$ powder, for experimental conditions see Table 3 ). The catalyst was packed in the central 6 inch section of the tube reactor, which is 18 inches long. Different structures of reactor tube were designed to hold the catalysts as shown in Figure 2. For $\mathrm{Ni}-\mathrm{MO} / \mathrm{Al}_{2} \mathrm{O}_{3}$, the catalyst was preceded in the reactor by 6 inches of $3 \mathrm{~mm}$ glass beads and followed by 6 inches of $3 \mathrm{~mm}$ glass beads which was supported on glass wool. Fe-l catalyst was held in a basket made of 400 mesh stainless steel screen and the glass beads were the same as that for $\mathrm{Ni-MO}$. A $0.5 \mu \mathrm{m}$ stainless steel frit was used to hold $\mathrm{Fe}-2$ catalyst with the glass beads preceding the catalyst of $0.1-0.12 \mathrm{~mm}$ and nothing following.

In all the experiments, the reactor was operated at about $1000 \mathrm{psi}$ at a hydrogen flow rate of $100 \mathrm{ml} / \mathrm{min}$ (STP) which was in large stoichiometric excess. Nitrogen was used to purge and pressurize the reactor before starting the reaction. The reactant feed solution contained 2 wt $z$ naphthalene (Fisher, >99\%) in mineral oil (Hunco). The liquid feedstock flow rate was $0.2 \mathrm{ml} / \mathrm{min}$ 
except for the Ni-Mo catalyst, in which case different flow rates were tested. Temperature was varied from $200^{\circ} \mathrm{C}$ to $400^{\circ} \mathrm{C}$ to examine the dependence of activity on the reaction conditions. A relative activity $A_{H}$ and a cracking activity $A_{C}$ were defined as follows to characterize the extent of the hydrogenation and cracking reactions, respectively.

$$
\begin{aligned}
& A_{H^{-}} \frac{2 * M_{\mathrm{T}}+5 * M_{D}}{5 *\left(M_{N}+M_{T}+M_{D}\right)} \\
& A_{C}-1-\frac{\left(M_{D}+M_{T^{2}}+M_{N}\right)}{M_{N o}}
\end{aligned}
$$

Where, $\mathrm{M}_{W}$ : moles of naphthalene (NAPH) in $100 \mathrm{~g}$ liquid product $M_{\mathrm{T}}$ : moles of tetralin (TET) in $100 \mathrm{~g}$ liguid product $M_{0}$ : moles of decalin (DEC) in $100 \mathrm{~g}$ liquid product $\mathrm{M}_{\mathrm{NO}}$ : moles of $\mathrm{NAPH}$ in $100 \mathrm{~g}$ liquid feed

The product samples were sampled periodically from the reactor effluent and analyzed with a Varian 3300 gas chromatograph. Temperature programming was used to improve peak resolution and decane was used as an internal standard.

\section{RESULTS AND DISCUSSION}

Ten experimental runs with catalyst were carried out: one with Ni-Mo, two with Fe-1 and the other seven with Fe-2. In addition, a blank test was performed at temperatures up to $400^{\circ} \mathrm{C}$ and showed 
that the reactor filled with glass beads alone had negligible activity for naphthalene hydrogenation. In many of the experiments, especially at high temperature, the total moles of NAPH, TET and DEC were less than the moles of NAPH feed. We suggest that the missing material probably results from (1) undetected reactions giving volatile gaseous products and coke deposits on the catalyst, (2) unidentified products which were mixed with the cracking products of mineral oil at high temperature. This is the basis for our definition of cracking activity $A_{c}$ in equation (2).

Ni-Mo (Run $\$ 1$ ) This reaction was used to test the equipment and as a basis of comparison for later runs. Ten different reaction conditions were selected for Ni-Mo catalyst in a total run of 864 hrs. Table 1 shows the wt $f$ of NAPH, TET and DEC in the liquid product. No obvious deactivation was observed since the relative activity was about the same under identical conditions after 768 hrs, even though a fairly severe condition $\left(425^{\circ} \mathrm{C}\right)$ was employed. cracking activity was fairly low except at the upper temperature $400^{\circ} \mathrm{C}$ where it was about $10 \%$ of the NAPH feed. It can be seen from Fig 3 , in which we have plotted the relative activity $A_{H}$ vs. temperature at the liquid flow rate of $0.2 \mathrm{ml} / \mathrm{min}$, that an optimum temperature existed $\left(-310^{\circ} \mathrm{C}\right)$. We suggest that this results from the thermodynamics of the reactions. Low temperature favors the exothermic hydrogenation reactions, but at too low a temperature, the reaction rate is slower. At $300^{\circ} \mathrm{C}, A_{H}$ decreased with the increase of liquid flow rate (Figure 4 ). This phenomenon can be 
Table 1. Results of Product Analyses for Ni-Mo Catalyst (Run 1 )

\begin{tabular}{|c|c|c|c|c|c|c|c|}
\hline & $\begin{array}{l}\text { Temp }\left({ }^{\circ} \mathrm{C}\right) \\
\text { \& }{ }_{\text {quid }} \\
\text { rate } \\
(\mathrm{m} 1 / \mathrm{min})\end{array}$ & $\begin{array}{l}\text { Time } \\
\text { (hrs) }\end{array}$ & $\begin{array}{l}\text { DEC } \\
\text { wt \& }\end{array}$ & $\begin{array}{l}\text { TET } \\
\text { wt } \&\end{array}$ & $\begin{array}{l}\text { NAPH } \\
\text { wt ? }\end{array}$ & $A_{H}$ & $A_{c}$ \\
\hline $\begin{array}{l}1 \\
2 \\
3 \\
4 \\
5\end{array}$ & $\begin{array}{c}400^{\circ} \mathrm{C} \\
0.2 \mathrm{ml} / \mathrm{min}\end{array}$ & $\begin{array}{r}24 \\
72 \\
96 \\
120 \\
144\end{array}$ & $\begin{array}{l}1.47 \\
1.11 \\
1.04 \\
1.06 \\
1.10\end{array}$ & $\begin{array}{l}0.29 \\
0.89 \\
0.79 \\
0.78 \\
0.77\end{array}$ & $\begin{array}{l}0.01 \\
0.04 \\
0.04 \\
0.04 \\
0.04\end{array}$ & $\begin{array}{l}0.89 \\
0.71 \\
0.72 \\
0.72 \\
0.73\end{array}$ & $\begin{array}{l}0.18 \\
0.03 \\
0.11 \\
0.12 \\
0.10\end{array}$ \\
\hline $\begin{array}{r}6 \\
7 \\
8 \\
9 \\
10 \\
11\end{array}$ & $\begin{array}{c}300^{\circ} \mathrm{C} \\
0.2 \mathrm{ml} / \mathrm{min}\end{array}$ & $\begin{array}{l}150 \\
166 \\
171 \\
178 \\
192 \\
216\end{array}$ & $\begin{array}{l}1.90 \\
1.88 \\
1.72 \\
1.83 \\
1.94 \\
1.91\end{array}$ & $\begin{array}{l}0.26 \\
0.25 \\
0.23 \\
0.24 \\
0.25 \\
0.24\end{array}$ & $\begin{array}{l}0.00 \\
0.00 \\
0.00 \\
0.00 \\
0.00 \\
0.00\end{array}$ & $\begin{array}{l}0.93 \\
0.93 \\
0.93 \\
0.93 \\
0.93 \\
0.93\end{array}$ & $\begin{array}{r}-0.01 \\
0.01 \\
0.09 \\
0.04 \\
-0.02 \\
0.00\end{array}$ \\
\hline $\begin{array}{l}12 \\
13\end{array}$ & $\begin{array}{c}300^{\circ} \mathrm{C} \\
0.4 \mathrm{ml} / \mathrm{min}\end{array}$ & $\begin{array}{l}226 \\
240\end{array}$ & $\begin{array}{l}1.17 \\
1.23\end{array}$ & $\begin{array}{l}0.81 \\
0.79\end{array}$ & $\begin{array}{l}0.00 \\
0.00\end{array}$ & $\begin{array}{l}0.75 \\
0.76\end{array}$ & $\begin{array}{l}0.06 \\
0.04\end{array}$ \\
\hline $\begin{array}{l}14 \\
15 \\
16 \\
17\end{array}$ & $\begin{array}{c}200^{\circ} \mathrm{C} \\
0.2 \mathrm{ml} / \mathrm{min}\end{array}$ & $\begin{array}{l}264 \\
288 \\
312 \\
336\end{array}$ & $\begin{array}{l}0.75 \\
0.74 \\
0.80 \\
0.79\end{array}$ & $\begin{array}{l}1.07 \\
1.15 \\
1.28 \\
1.29\end{array}$ & $\begin{array}{l}0.07 \\
0.10 \\
0.14 \\
0.13\end{array}$ & $\begin{array}{l}0.62 \\
0.60 \\
0.58 \\
0.58\end{array}$ & $\begin{array}{r}0.10 \\
0.05 \\
-0.06 \\
-0.06\end{array}$ \\
\hline $\begin{array}{l}18 \\
19 \\
20\end{array}$ & $\begin{array}{c}250^{\circ} \mathrm{C} \\
0.2 \mathrm{ml} / \mathrm{min}\end{array}$ & $\begin{array}{l}360 \\
384 \\
408\end{array}$ & $\begin{array}{l}1.46 \\
1.51 \\
1.44\end{array}$ & $\begin{array}{l}0.55 \\
0.59 \\
0.56\end{array}$ & $\begin{array}{l}0.01 \\
0.01 \\
0.01\end{array}$ & $\begin{array}{l}0.83 \\
0.82 \\
0.82\end{array}$ & $\begin{array}{l}0.05 \\
0.00 \\
0.05\end{array}$ \\
\hline $\begin{array}{l}21 \\
22 \\
23\end{array}$ & $\begin{array}{c}300^{\circ} \mathrm{C} \\
0.2 \mathrm{ml} / \mathrm{min}\end{array}$ & $\begin{array}{l}432 \\
456 \\
480\end{array}$ & $\begin{array}{l}1.78 \\
1.88 \\
1.70\end{array}$ & $\begin{array}{l}0.28 \\
0.30 \\
0.27\end{array}$ & $\begin{array}{l}0.00 \\
0.00 \\
0.00\end{array}$ & $\begin{array}{l}0.92 \\
0.92 \\
0.92\end{array}$ & $\begin{array}{r}0.04 \\
-0.02 \\
0.08\end{array}$ \\
\hline $\begin{array}{l}24 \\
25 \\
26\end{array}$ & $\begin{array}{c}350^{\circ} \mathrm{C} \\
0.2 \mathrm{ml} / \mathrm{min}\end{array}$ & $\begin{array}{l}504 \\
528 \\
552\end{array}$ & $\begin{array}{l}1.53 \\
1.68 \\
1.48\end{array}$ & $\begin{array}{l}0.33 \\
0.34 \\
0.44\end{array}$ & $\begin{array}{l}0.00 \\
0.00 \\
0.04\end{array}$ & $\begin{array}{l}0.89 \\
0.90 \\
0.84\end{array}$ & $\begin{array}{l}0.13 \\
0.06 \\
0.08\end{array}$ \\
\hline $\begin{array}{l}27 \\
28 \\
29\end{array}$ & $\begin{array}{c}425^{\circ} \mathrm{C} \\
0.2 \mathrm{ml} / \mathrm{min}\end{array}$ & $\begin{array}{l}576 \\
600 \\
624\end{array}$ & $\begin{array}{l}0.72 \\
0.64 \\
0.65\end{array}$ & $\begin{array}{l}1.19 \\
1.15 \\
1.09\end{array}$ & $\begin{array}{l}0.22 \\
0.17 \\
1.18\end{array}$ & $\begin{array}{l}0.55 \\
0.55 \\
0.56\end{array}$ & $\begin{array}{r}-0.02 \\
0.06 \\
0.08\end{array}$ \\
\hline $\begin{array}{l}30 \\
31 \\
32 \\
33\end{array}$ & $\begin{array}{c}325^{\circ} \mathrm{C} \\
0.2 \mathrm{ml} / \mathrm{min}\end{array}$ & $\begin{array}{l}648 \\
672 \\
696 \\
720\end{array}$ & $\begin{array}{l}1.74 \\
1.86 \\
1.72 \\
1.88\end{array}$ & $\begin{array}{l}0.23 \\
0.21 \\
0.19 \\
0.20\end{array}$ & $\begin{array}{l}0.00 \\
0.00 \\
0.00 \\
0.00\end{array}$ & $\begin{array}{l}0.93 \\
0.94 \\
0.94 \\
0.94\end{array}$ & $\begin{array}{l}0.08 \\
0.04 \\
0.11 \\
0.03\end{array}$ \\
\hline $\begin{array}{l}34 \\
35\end{array}$ & $\begin{array}{c}400^{\circ} \mathrm{C} \\
0.2 \mathrm{ml} / \mathrm{min}\end{array}$ & $\begin{array}{l}744 \\
768\end{array}$ & $\begin{array}{l}1.07 \\
1.14\end{array}$ & $\begin{array}{l}0.72 \\
0.70\end{array}$ & $\begin{array}{l}0.04 \\
0.04\end{array}$ & $\begin{array}{l}0.73 \\
0.75\end{array}$ & $\begin{array}{l}0.13 \\
0.11\end{array}$ \\
\hline $\begin{array}{l}36 \\
37\end{array}$ & $\begin{array}{c}300^{\circ} \mathrm{C} \\
0.3 \mathrm{ml} / \mathrm{min}\end{array}$ & $\begin{array}{l}792 \\
816\end{array}$ & $\begin{array}{l}1.89 \\
1.80\end{array}$ & $\begin{array}{l}0.56 \\
0.50\end{array}$ & $\begin{array}{l}0.00 \\
0.00\end{array}$ & $\begin{array}{l}0.86 \\
0.86\end{array}$ & $\begin{array}{l}-0.15 \\
-0.08\end{array}$ \\
\hline $\begin{array}{l}38 \\
39\end{array}$ & $\begin{array}{c}300^{\circ} \mathrm{C} \\
0.15 \mathrm{ml} / \mathrm{min}\end{array}$ & $\begin{array}{l}840 \\
864\end{array}$ & $\begin{array}{l}1.84 \\
1.95\end{array}$ & $\begin{array}{l}0.05 \\
0.06\end{array}$ & $\begin{array}{l}0.00 \\
0.00\end{array}$ & $\begin{array}{l}0.98 \\
0.98\end{array}$ & $\begin{array}{l}0.12 \\
0.07\end{array}$ \\
\hline
\end{tabular}




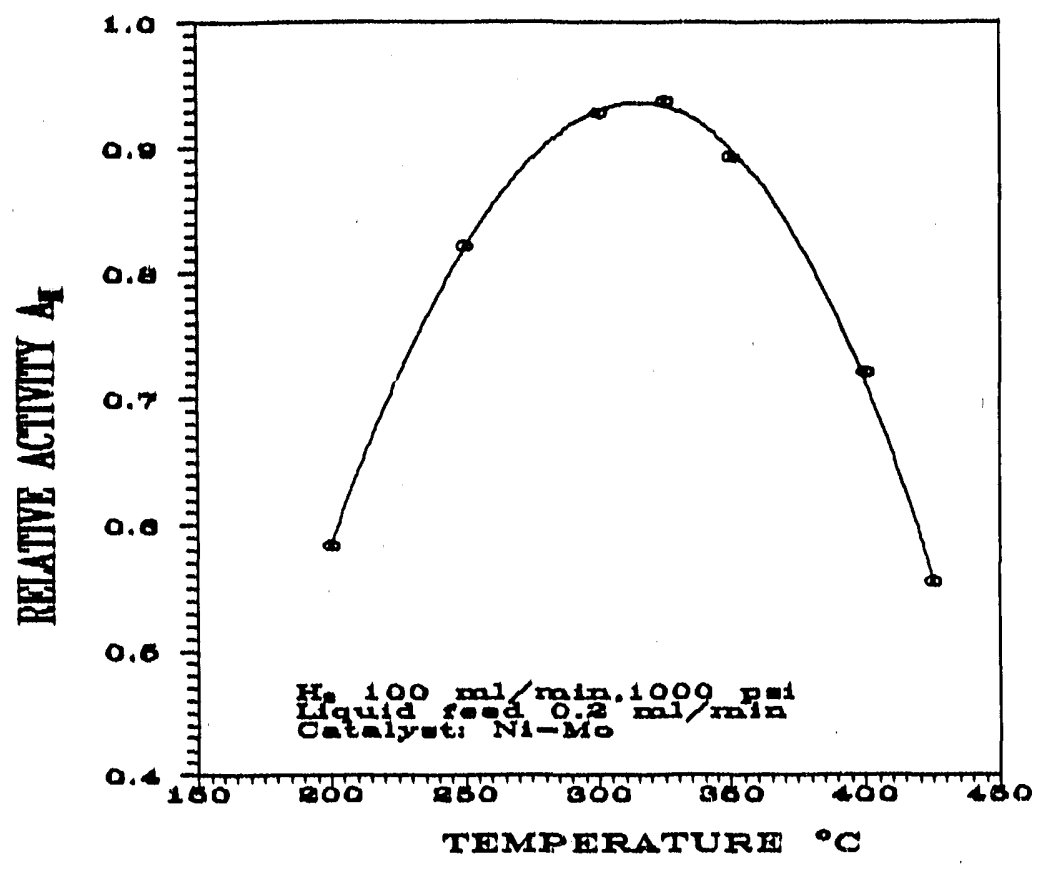

Figure 3. The dependence of $A_{n}$ on temperature

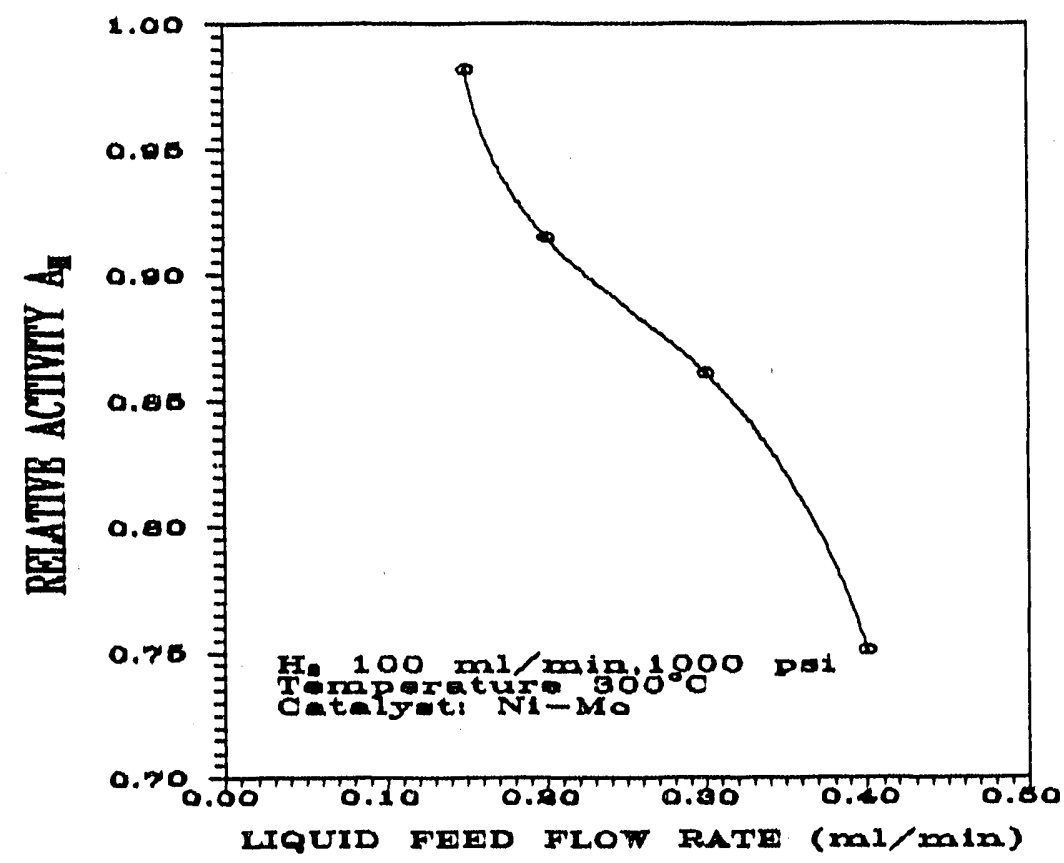

Pigure 1. The dependence of $A_{H}$ on liquid flow rate 
Table 2. Results of Product Analyses for Pe-1 (Harshaw) Catalyst (Run \$2)

\begin{tabular}{|c|c|c|c|c|c|c|c|}
\hline & $\begin{array}{l}\text { Temp } \\
\left({ }^{\circ} \mathrm{C}\right)\end{array}$ & $\begin{array}{l}\text { Time } \\
\text { (hrs) }\end{array}$ & $\begin{array}{l}\text { DEC } \\
\text { (wt } z \text { ) }\end{array}$ & $\begin{array}{l}\text { TEI } \\
\text { (wt \&) }\end{array}$ & $\begin{array}{l}\text { NAPH } \\
\text { (wt \&) }\end{array}$ & $A_{H}$ & $A_{c}$ \\
\hline $\begin{array}{l}1 \\
2 \\
3 \\
4\end{array}$ & 400 & $\begin{array}{l}24 \\
48 \\
72 \\
96\end{array}$ & $\begin{array}{l}0.21 \\
0.31 \\
0.45 \\
0.19\end{array}$ & $\begin{array}{l}0.22 \\
0.38 \\
0.49 \\
0.44\end{array}$ & $\begin{array}{l}0.31 \\
0.34 \\
0.35 \\
0.28\end{array}$ & $\begin{array}{l}0.39 \\
0.44 \\
0.49 \\
0.40\end{array}$ & $\begin{array}{l}0.64 \\
0.50 \\
0.38 \\
0.56\end{array}$ \\
\hline $\begin{array}{r}5 \\
6 \\
7 \\
8 \\
9 \\
10\end{array}$ & 300 & $\begin{array}{l}120 \\
144 \\
168 \\
192 \\
216 \\
240\end{array}$ & $\begin{array}{l}0.34 \\
0.29 \\
0.14 \\
0.07 \\
0.00 \\
0.00\end{array}$ & $\begin{array}{l}0.97 \\
0.38 \\
0.28 \\
0.21 \\
0.16 \\
0.10\end{array}$ & $\begin{array}{l}0.70 \\
1.32 \\
1.48 \\
1.69 \\
1.85 \\
1.86\end{array}$ & $\begin{array}{l}0.35 \\
0.21 \\
0.13 \\
0.07 \\
0.03 \\
0.02\end{array}$ & $\begin{array}{r}0.02 \\
0.02 \\
0.06 \\
0.03 \\
-0.01 \\
0.02\end{array}$ \\
\hline $\begin{array}{l}11 \\
12 \\
13\end{array}$ & 350 & $\begin{array}{l}246 \\
264 \\
288\end{array}$ & $\begin{array}{l}0.00 \\
0.00 \\
0.00\end{array}$ & $\begin{array}{l}0.12 \\
0.10 \\
0.08\end{array}$ & $\begin{array}{l}1.90 \\
1.85 \\
1.88\end{array}$ & $\begin{array}{l}0.02 \\
0.02 \\
0.02\end{array}$ & $\begin{array}{r}-0.01 \\
0.02 \\
0.02\end{array}$ \\
\hline $\begin{array}{l}14 \\
15 \\
16\end{array}$ & 375 & $\begin{array}{l}312 \\
336 \\
360\end{array}$ & $\begin{array}{l}0.00 \\
0.00 \\
0.00\end{array}$ & $\begin{array}{l}0.08 \\
0.05 \\
0.05\end{array}$ & $\begin{array}{l}1.93 \\
1.83 \\
2.03\end{array}$ & $\begin{array}{l}0.02 \\
0.01 \\
0.01\end{array}$ & $\begin{array}{r}0.00 \\
0.06 \\
-0.04\end{array}$ \\
\hline $\begin{array}{l}17 \\
18\end{array}$ & 400 & $\begin{array}{l}384 \\
408\end{array}$ & $\begin{array}{l}0.00 \\
0.00\end{array}$ & $\begin{array}{l}0.05 \\
0.08\end{array}$ & $\begin{array}{l}1.82 \\
1.87\end{array}$ & $\begin{array}{l}0.01 \\
0.02\end{array}$ & $\begin{array}{l}0.07 \\
0.03\end{array}$ \\
\hline
\end{tabular}

(Run $\$ 3)$

\begin{tabular}{|c|c|c|c|c|c|c|c|}
\hline & $\begin{array}{l}\text { Temp } \\
\text { ( } \mathrm{C})\end{array}$ & $\begin{array}{l}\text { Time } \\
\text { (hrs) }\end{array}$ & $\begin{array}{l}\text { DEC } \\
\text { (wt \&) }\end{array}$ & $\begin{array}{c}\text { TET } \\
(w t \&)\end{array}$ & $\begin{array}{l}\text { NAPH } \\
\text { (wt } 8)\end{array}$ & $A_{H}$ & $A_{C}$ \\
\hline $\begin{array}{l}1 \\
2 \\
3 \\
4\end{array}$ & 300 & $\begin{array}{l}24 \\
48 \\
72 \\
96\end{array}$ & $\begin{array}{l}1.94 \\
2.01 \\
2.19 \\
2.06\end{array}$ & $\begin{array}{l}0.00 \\
0.00 \\
0.00 \\
0.00\end{array}$ & $\begin{array}{l}0.00 \\
0.00 \\
0.00 \\
0.00\end{array}$ & $\begin{array}{l}1.00 \\
1.00 \\
1.00 \\
1.00\end{array}$ & $\begin{array}{r}0.10 \\
0.07 \\
-0.02 \\
0.05\end{array}$ \\
\hline $\begin{array}{l}5 \\
6 \\
7 \\
8\end{array}$ & 350 & $\begin{array}{l}101 \\
120 \\
144 \\
151\end{array}$ & $\begin{array}{l}1.88 \\
1.78 \\
1.78 \\
1.61\end{array}$ & $\begin{array}{l}0.03 \\
0.03 \\
0.06 \\
0.08\end{array}$ & $\begin{array}{l}0.00 \\
0.00 \\
0.00 \\
0.00\end{array}$ & $\begin{array}{l}0.99 \\
0.99 \\
0.98 \\
0.97\end{array}$ & $\begin{array}{l}0.11 \\
0.16 \\
0.15 \\
0.21\end{array}$ \\
\hline $\begin{array}{r}9 \\
10 \\
11\end{array}$ & 400 & $\begin{array}{l}168 \\
192 \\
216\end{array}$ & $\begin{array}{l}0.37 \\
0.07 \\
0.02\end{array}$ & $\begin{array}{l}0.48 \\
0.53 \\
0.19\end{array}$ & $\begin{array}{l}0.53 \\
0.76 \\
1.41\end{array}$ & $\begin{array}{l}0.40 \\
0.20 \\
0.06\end{array}$ & $\begin{array}{l}0.33 \\
0.33 \\
0.20\end{array}$ \\
\hline 12 & 300 & 240 & 0.00 & 0.21 & 1.69 & 0.04 & 0.06 \\
\hline
\end{tabular}


attributed to the decrease of residence time as the flow rate is increased.

Fe-1 (Harabaw, Runs $\$ 2,33)$ The reaction Run \#2 with Fe-1 catalyst was begun first at $400^{\circ} \mathrm{C}$ (Table 2, Run 12 ). Low $A_{H}$ was observed and it kept decreasing although the temperature had been changed to $300^{\circ} \mathrm{C}$. Therefore, the reaction was repeated (Run 3 ) with $300^{\circ} \mathrm{C}$ as the starting temperature. The relative activity was so high that neither unreacted naphthalene nor tetralin was detected. When the temperature was increased to $400^{\circ} \mathrm{C}, A_{H}$ decreased to about zero again and activity could not be restored upon returning the temperature to $300^{\circ} \mathrm{C}$. These results obviously show that, in both runs, the catalyst has undergone a rapid deactivation by coking from the cracking of naphthalene. About half of the reactant naphthalene was not found in any products and a number of unidentified lighter components appeared on GC output. As for the activity decrease at $300^{\circ} \mathrm{C}$ in the Run \#3, it was probable that during the preceding 96 hrs at $400^{\circ} \mathrm{C}$, the catalyst was coked, resulting in the activity 10ss. In both runs the cracking activity was high $(30-50$ \%) at $400^{\circ} \mathrm{C}$, especially in Run $\$ 2$ with the fresh catalyst.

Fo-2 (8tram, Runs $\$ 1--\$ 10)$ Seven runs were performed with $\mathrm{Fe}_{2} \mathrm{O}_{3}$ powder catalyst from strem Chemical Inc. The different reaction conditions are shown in Table 3. Run \#4 (Table 4) shows evidence of catalyst deactivation throughout the run, while Run \#5 has approximately constant activity. Both runs show little activity in 
Table 3. Reaction Conditions with re-2 Catalyst

\begin{tabular}{|c|c|c|c|c|c|}
\hline $\begin{array}{l}\text { Run } \\
\text { No }\end{array}$ & $\begin{array}{l}\text { Initial } \\
\text { Temp. }\left({ }^{\circ} \mathrm{C}\right)\end{array}$ & $\begin{array}{l}\text { Temp. Range } \\
\left({ }^{\circ} \mathrm{C}\right)\end{array}$ & $\begin{array}{c}\text { cat.wt. } \\
\text { (g) }\end{array}$ & $\begin{array}{r}\mathrm{H}_{2} \\
\text { Reduction }\end{array}$ & $\begin{array}{c}C_{2} \\
\text { In Feed } \\
\end{array}$ \\
\hline 4 & 250 & $250--400$ & 22.81 & No & No \\
\hline 5 & 300 & $300--400$ & 21.84 & No & No \\
\hline 6 & 250 & $250--400$ & 22.13 & Yes & No \\
\hline 7 & 200 & $200--350$ & 22.84 & Yes & No \\
\hline 8 & 250 & const. 250 & 22.85 & No & $\begin{array}{c}\text { last } \\
32 \text { hrs }\end{array}$ \\
\hline 9 & 250 & const. 250 & 22.59 & No & $\begin{array}{l}\text { first } \\
92 \text { hrs }\end{array}$ \\
\hline 10 & 250 & const. 250 & 22.64 & No & $\begin{array}{l}\text { first } \\
48 \text { hrs }\end{array}$ \\
\hline
\end{tabular}

the first 5 to 6 hours indicating an activation of the catalyst by $\mathrm{H}_{2}$ reduction. This view was supported by the results of Runs \#6, \#7 (Table 5) when the catalysts were $\mathrm{H}_{2}$ reduced before reaction and Run \#8 ( $7 \mathrm{ig} 5)$ in which the temperature was held constant at $250^{\circ} \mathrm{C}$ and samples taken every two hours during the first 24 hours. Material balances are somewhat better in Run \#5, where the sum of the weight percent products is about $2 \%$. Since the main difference in Run \#4 and Run \#5 is only the initial temperature, the results indicate that this initial activation temperature may be important for subsequent catalyst performance.

In order to further investigate the effect of reduction, two experimental runs (Table 5, Runs \$6, 7) were carried out with catalyst being partially reduced in a $\mathrm{H}_{2}$ atmosphere for $24 \mathrm{hrs}$ 
Table 4. Results of Product Analyses for: Fe-2 (strem) Catalyst (Run 14)

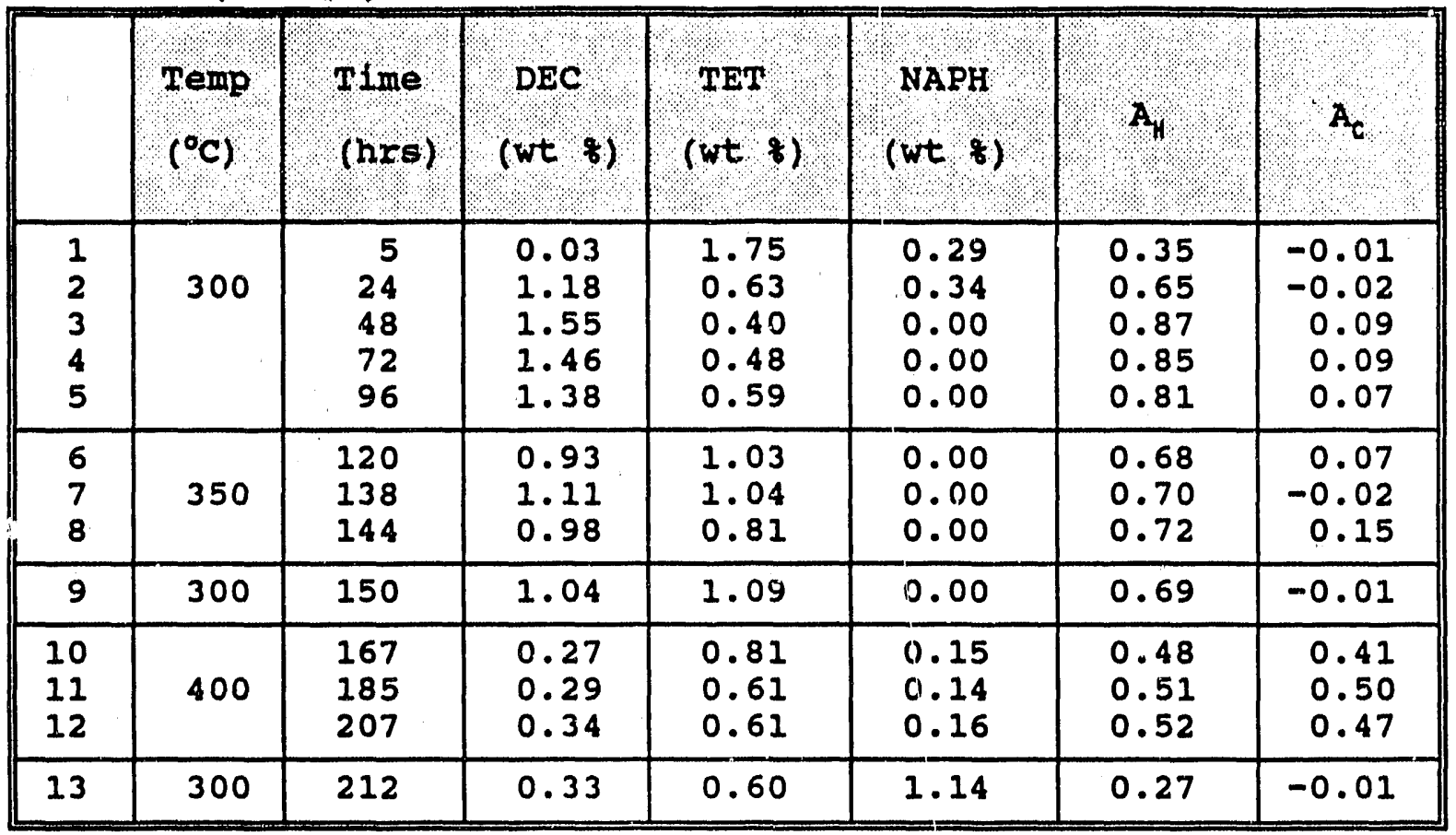

(Run \$5)

\begin{tabular}{|c|c|c|c|c|c|c|c|}
\hline & $\begin{array}{l}\text { Temp } \\
\left({ }^{\circ} \mathrm{C}\right)\end{array}$ & $\begin{array}{l}\text { Time } \\
\text { (hrs) }\end{array}$ & $\begin{array}{l}\text { DEC } \\
\text { (wt \&) }\end{array}$ & $\begin{array}{l}\text { TET } \\
\text { (wt \&) }\end{array}$ & $\begin{array}{l}\text { NAPH } \\
\text { (wt \&) }\end{array}$ & $A_{H}$ & $A_{c}$ \\
\hline $\begin{array}{l}1 \\
2\end{array}$ & 250 & $\begin{array}{r}6 \\
24\end{array}$ & $\begin{array}{l}0.00 \\
0.10\end{array}$ & $\begin{array}{l}0.01 \\
1.66\end{array}$ & $\begin{array}{l}2.06 \\
0.03\end{array}$ & $\begin{array}{l}0.00 \\
0.43\end{array}$ & $\begin{array}{r}-0.04 \\
0.13\end{array}$ \\
\hline $\begin{array}{l}3 \\
4\end{array}$ & 300 & $\begin{array}{l}29 \\
48\end{array}$ & $\begin{array}{l}0.13 \\
0.16\end{array}$ & $\begin{array}{l}2.05 \\
2.12\end{array}$ & $\begin{array}{l}0.0 .3 \\
0.00\end{array}$ & $\begin{array}{l}0.43 \\
0.44\end{array}$ & $\begin{array}{l}-0.07 \\
-0.10\end{array}$ \\
\hline $\begin{array}{l}5 \\
6\end{array}$ & 325 & $\begin{array}{l}53 \\
72\end{array}$ & $\begin{array}{l}0.11 \\
0.14\end{array}$ & $\begin{array}{l}1.94 \\
2.10\end{array}$ & $\begin{array}{l}0.00 \\
0.00\end{array}$ & $\begin{array}{l}0.43 \\
0.44\end{array}$ & $\begin{array}{r}0.01 \\
-0.09\end{array}$ \\
\hline $\begin{array}{l}7 \\
8\end{array}$ & 350 & $\begin{array}{l}77 \\
96\end{array}$ & $\begin{array}{l}0.11 \\
0.13\end{array}$ & $\begin{array}{l}2.00 \\
2.06\end{array}$ & $\begin{array}{l}0.01 \\
0.01\end{array}$ & $\begin{array}{l}0.43 \\
0.43\end{array}$ & $\begin{array}{l}-0.03 \\
-0.07\end{array}$ \\
\hline $\begin{array}{r}9 \\
10\end{array}$ & 375 & $\begin{array}{l}101 \\
120\end{array}$ & $\begin{array}{l}0.09 \\
0.09\end{array}$ & $\begin{array}{l}1.88 \\
1.91\end{array}$ & $\begin{array}{l}0.03 \\
0.05\end{array}$ & $\begin{array}{l}0.42 \\
0.42\end{array}$ & $\begin{array}{l}0.03 \\
0.01\end{array}$ \\
\hline $\begin{array}{l}11 \\
12\end{array}$ & 400 & $\begin{array}{l}125 \\
144\end{array}$ & $\begin{array}{l}0.14 \\
0.10\end{array}$ & $\begin{array}{l}1.60 \\
1.49\end{array}$ & $\begin{array}{l}0.07 \\
0.07\end{array}$ & $\begin{array}{l}0.43 \\
0.42\end{array}$ & $\begin{array}{l}0.12 \\
0.20\end{array}$ \\
\hline 13 & 300 & 150 & 0.10 & 1.89 & 0.08 & 0.41 & 0.00 \\
\hline
\end{tabular}


Table 5. Results of Product Analyses for Reduced Fe-2 Catalyst (Run (6)

\begin{tabular}{|c|c|c|c|c|c|c|c|}
\hline & $\begin{array}{l}\text { Temp } \\
\left({ }^{\circ} \mathrm{C}\right)\end{array}$ & $\begin{array}{l}\text { Time } \\
\text { (hrs) }\end{array}$ & $\begin{array}{c}D E C \\
(w t \quad z)\end{array}$ & $\begin{array}{c}\text { TET } \\
\text { (wt \&) }\end{array}$ & $\begin{array}{l}\text { NAPH } \\
\text { (wt \&) }\end{array}$ & $A_{H}$ & $A_{c}$ \\
\hline $\begin{array}{l}1 \\
2\end{array}$ & 250 & $\begin{array}{r}5 \\
24\end{array}$ & $\begin{array}{l}0.87 \\
0.70\end{array}$ & $\begin{array}{l}1.36 \\
1.36\end{array}$ & $\begin{array}{l}0.03 \\
0.02\end{array}$ & $\begin{array}{l}0.62 \\
0.59\end{array}$ & $\begin{array}{r}-0.07 \\
0.01\end{array}$ \\
\hline $\begin{array}{l}3 \\
4\end{array}$ & 300 & $\begin{array}{l}29 \\
48\end{array}$ & $\begin{array}{l}1.37 \\
1.84\end{array}$ & $\begin{array}{l}0.61 \\
0.56\end{array}$ & $\begin{array}{l}0.00 \\
0.00\end{array}$ & $\begin{array}{l}0.81 \\
0.85\end{array}$ & $\begin{array}{r}0.07 \\
-0.12\end{array}$ \\
\hline $\begin{array}{l}5 \\
6\end{array}$ & 325 & $\begin{array}{l}53 \\
72\end{array}$ & $\begin{array}{l}1.63 \\
1.55\end{array}$ & $\begin{array}{l}0.28 \\
0.28\end{array}$ & $\begin{array}{l}0.00 \\
0.00\end{array}$ & $\begin{array}{l}0.91 \\
0.90\end{array}$ & $\begin{array}{l}0.11 \\
0.15\end{array}$ \\
\hline $\begin{array}{l}7 \\
8\end{array}$ & 350 & $\begin{array}{l}77 \\
96\end{array}$ & $\begin{array}{l}1.42 \\
1.56\end{array}$ & $\begin{array}{l}0.33 \\
0.30\end{array}$ & $\begin{array}{l}0.02 \\
0.01\end{array}$ & $\begin{array}{l}0.87 \\
0.89\end{array}$ & $\begin{array}{l}0.17 \\
0.13\end{array}$ \\
\hline $\begin{array}{r}9 \\
10\end{array}$ & 375 & $\begin{array}{l}101 \\
120\end{array}$ & $\begin{array}{l}0.75 \\
0.79\end{array}$ & $\begin{array}{l}0.32 \\
0.32\end{array}$ & $\begin{array}{l}0.08 \\
0.06\end{array}$ & $\begin{array}{l}0.76 \\
0.78\end{array}$ & $\begin{array}{l}0.46 \\
0.45\end{array}$ \\
\hline $\begin{array}{l}11 \\
12\end{array}$ & 400 & $\begin{array}{l}125 \\
144\end{array}$ & $\begin{array}{l}0.49 \\
0.36\end{array}$ & $\begin{array}{l}0.26 \\
0.41\end{array}$ & $\begin{array}{l}0.42 \\
0.12\end{array}$ & $\begin{array}{l}0.49 \\
0.58\end{array}$ & $\begin{array}{l}0.44 \\
0.57\end{array}$ \\
\hline 13 & 300 & 149 & 0.68 & 1.14 & 0.01 & 0.61 & 0.13 \\
\hline
\end{tabular}

(Run \$7)

\begin{tabular}{|r|c|c|c|c|c|c|c||}
\hline & $\begin{array}{c}\text { Temp } \\
\left({ }^{\circ} \mathrm{C}\right)\end{array}$ & $\begin{array}{c}\text { TIme } \\
\text { (hrs) }\end{array}$ & $\begin{array}{c}\text { DEC } \\
\text { (wt 8) }\end{array}$ & $\begin{array}{c}\text { TET } \\
\text { (wt 8) }\end{array}$ & $\begin{array}{c}\text { NAPH } \\
(\text { wt 8) }\end{array}$ & $A_{H}$ & $A_{C}$ \\
\hline 1 & 200 & 16 & 0.19 & 1.79 & 0.12 & 0.43 & -0.02 \\
2 & & 24 & 0.19 & 1.78 & 0.12 & 0.43 & -0.01 \\
\hline 3 & 250 & 29 & 1.06 & 1.03 & 0.02 & 0.69 & 0.00 \\
4 & & 48 & 1.14 & 0.93 & 0.00 & 0.72 & 0.02 \\
\hline 5 & 300 & 53 & 1.77 & 0.31 & 0.00 & 0.91 & 0.03 \\
6 & & 72 & 1.89 & 0.27 & 0.00 & 0.92 & -0.01 \\
\hline 7 & 325 & 77 & 1.79 & 0.19 & 0.00 & 0.94 & 0.08 \\
8 & & 96 & 1.85 & 0.17 & 0.00 & 0.95 & 0.06 \\
\hline 9 & 350 & 101 & 1.45 & 0.23 & 0.01 & 0.91 & 0.21 \\
10 & & 120 & 1.44 & 0.19 & 0.01 & 0.92 & 0.24 \\
\hline 11 & 200 & 125 & 0.37 & 1.53 & 0.05 & 0.50 & 0.06 \\
\hline
\end{tabular}


before starting the liquid feed. The reduction conditions were 1000 psi, $400^{\circ} \mathrm{C}$, with $\mathrm{H}_{2}$ flow rate $100 \mathrm{ml} / \mathrm{min}$ (STP). Catalyst in Run \#7 was cooled down in the same $\mathrm{H}_{2}$, atmosphere at $200^{\circ} \mathrm{C}$ for $1 \mathrm{hr}$ while catalyst in Run \#6 wasn't. It can be seen from Table 5 that partial reduction of catalyst will generally yield higher relative activity, although the temperature causing significant cracking is moved up to $350^{\circ} \mathrm{C}$ rather than $400^{\circ} \mathrm{C}$ observed before. The slightly higher $A_{H}$ in Run \#7 can be attributed to the additional reduction of catalyst in the cooling down process even though the temperature was lower $\left(200^{\circ} \mathrm{C}\right)$. Comparing the behavior of catalyst with and without reduction, it can be concluded that unsupported iron oxide in the ferric state will probably have negligible hydrogenation activity. Proper reduction to its metallic or ferrous state is required to activate its surface. $300^{\circ} \mathrm{C}$ should be a suitable reaction temperature from the consideration of both thermodynamics and cracking.

The test results with sulfur in the feed are shown in Fig 5. The source of sulfur is in the form of carbon disulfide which converts to $\mathrm{H}_{2} \mathrm{~S}$ instantly under the reaction condition. The amount of $\mathrm{CS}_{2}$ in the feed was 2.375 wto to give a concentration of sulfur of 2 wtr. The liquid feed without $\mathrm{CS}_{2}$ was charged in Run \#8 for the first 72 hrs and then switched to the feed with $\mathrm{CS}_{2}$, when the relative activity reached a relatively stable level. The $A_{H}$ dropped continuously to about zero after 32 hrs during this process indicating that the hydrogenation reactions have been inhibited by 


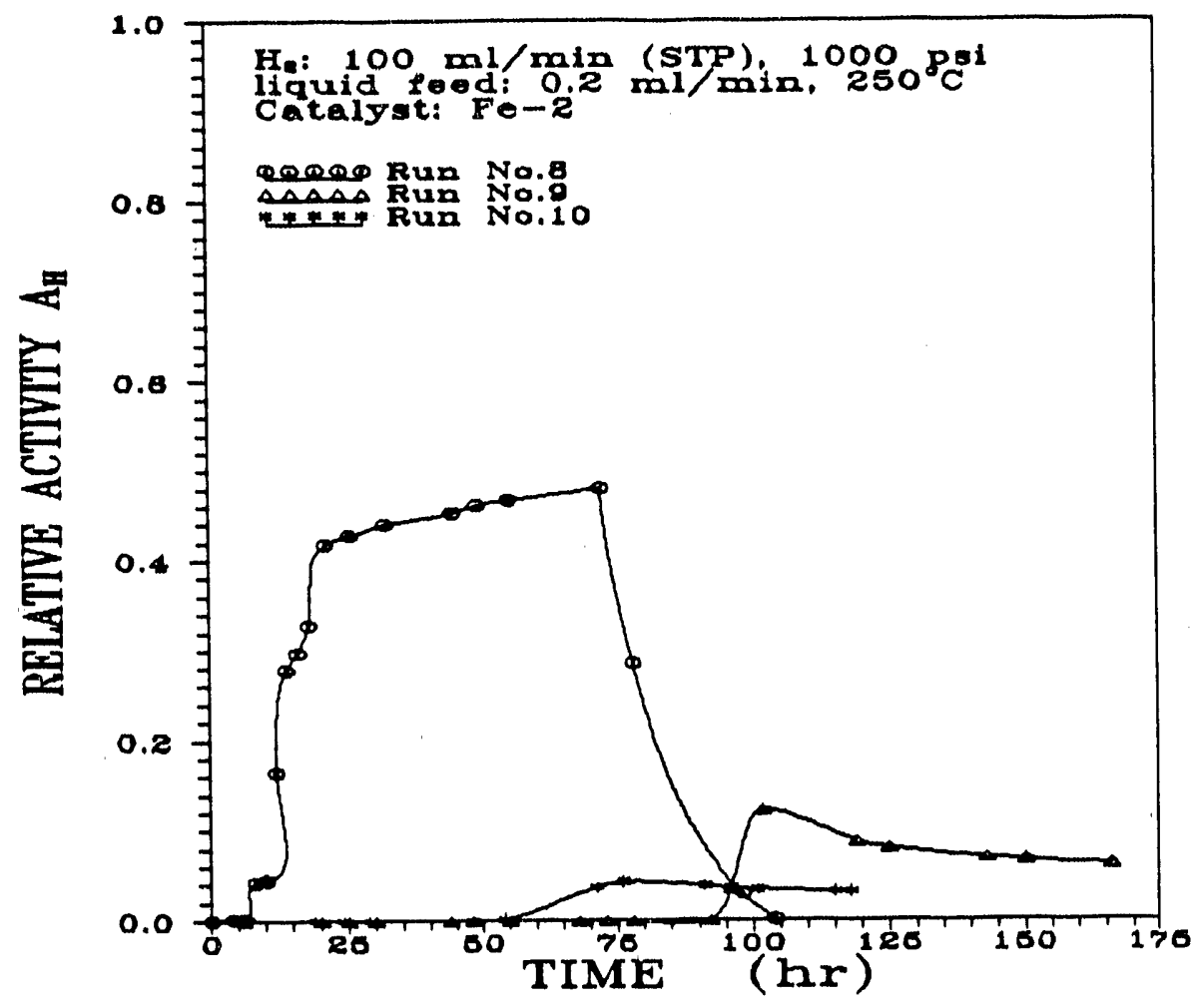

Figure 5. Relative Activity $A_{H}$ Vs. Time at $250^{\circ} \mathrm{C}$

the presence of $\mathrm{CS}_{2}$. Two more runs were performed by charging the feed with $\mathrm{CS}_{2}$ in the first $92 \mathrm{hrs}$ (Run $\$ 9$ ) and 48 hrs (Run 10 ) respectively, and normal feed was then provided after that. The results showed no reaction at all once there was $\mathrm{CS}_{2}$ in the feed, and $A_{H}$ could not be restored to its normal level. This obviously indicates that $\mathrm{H}_{2} \mathrm{~S}$ has poisoned the iron catalyst permanently for the hydrogenation reaction, because both runs showed very small, but stable $A_{H}$ although the residual $\mathrm{H}_{2}$ S concentration in the reactor has become less and less. This inhibition effect of $\mathrm{H}_{2} S$ is 
consistent with the earlier works by $Y . W$. Rhee et al [2] with naphthalene hydrogenation in batch reactor using $\mathrm{Ni-MO} / \mathrm{Al}_{2} \mathrm{O}_{3}$ catalyst and A.V. Sapre ${ }^{[3]}$ with biphenyl hydrogenation in the continuous reactor using $\mathrm{Co}-\mathrm{MO} / \mathrm{Al}_{2} \mathrm{O}_{3}$ catalyst.

\section{REFERENCES:}

1. Weng-Ping Wang and James A. Guin, FUEL PROCEssiNg TECHNOLOGY, 28 (1991), pp 149-166.

2. Young-Woo Rhee, James A. Guin, and Christine W. Curtis, POEL PROCE8BING TEChNOLOGY, 19 (1988), pp 1-5.

3. A.V. Sapre and B.C. Gates, IND. ENG.CHEM. RROCES8 DES. DEV. 21 (1982), pp 86-94. 

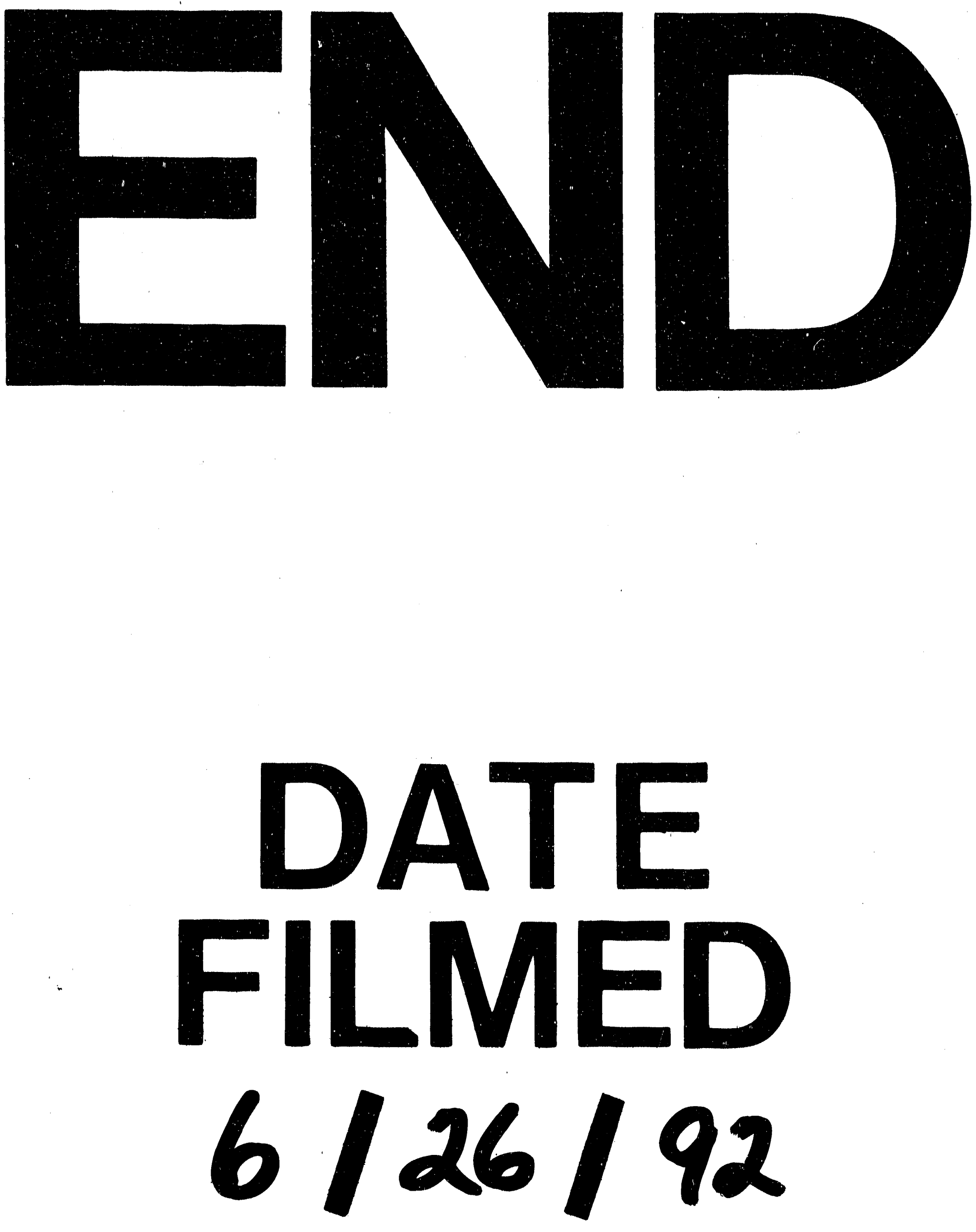

$6 / 26 / 92$ 
